\title{
OPTIMAL CONTROL OF A VLASOV-POISSON PLASMA BY AN EXTERNAL MAGNETIC FIELD
}

\author{
Patrik Knopf \\ University of Bayreuth
}





\title{
OPTIMAL CONTROL OF A VLASOV-POISSON PLASMA BY AN EXTERNAL MAGNETIC FIELD
}

\author{
Von der Universität Bayreuth \\ zur Erlangung des Grades eines \\ Doktors der Naturwissenschaften (Dr. rer. nat.) \\ genehmigte Abhandlung \\ von \\ Patrik Knopf \\ aus Bayreuth
}

$\begin{array}{ll}\text { 1. Gutachter: } & \text { Prof. Dr. Gerhard Rein } \\ \text { 2. Gutachter: } & \text { Prof. Dr. Anton Schiela }\end{array}$

Tag der Einreichung: 23. Mai 2017

Tag des Kolloquiums: 18. September 2017 



\section{Abstract}

We consider the three dimensional Vlasov-Poisson system in the plasma physical case. It describes the time evolution of the distribution function of a very large number of electrically charged particles. Those particles move under the influence of a self-consistent electric field that is given by Poisson's equation.

Our intention is to control the distribution function of the plasma by an external magnetic field. At first we introduce the basics for variational calculus. Then we discuss two model problems where the distribution function is to be controlled in such a way that it matches a desired distribution function at a certain point of time as closely as possible. Those model problems will be analyzed with respect to the following topics:

- Existence of a globally optimal solution

- Necessary conditions of first order for locally optimal solutions

- Derivation of an optimality system

- Sufficient conditions of second order for locally optimal solutions

- Uniqueness of the optimal control under certain conditions 


\section{Contents}

$\begin{array}{llr}1 & \text { Introduction } & 9\end{array}$

2 Some important tools $\quad 13$

2.1 Gronwall's lemma and some generalizations . . . . . . . . . . . . . . 13

2.2 A generalization of Jensen's inequality . . . . . . . . . . . . . . . . . 15

2.3 Sobolev inequalities and continuous embeddings . . . . . . . . . . . . . 16

2.4 A relation of Sobolev spaces and Bochner spaces . . . . . . . . . . . . . 17

2.5 The Newtonian potential . . . . . . . . . . . . . . . . . . . 21

3 Admissible fields and the field-state operator $\quad 29$

3.1 The set of admissible fields . . . . . . . . . . . . . . . 30

3.2 The characteristic flow of the Vlasov equation . . . . . . . . . . . . 32

3.3 Classical solutions for smooth external fields . . . . . . . . . . . . . . 34

3.4 Strong solutions for admissible external fields . . . . . . . . . . . . . . . 41

4 Continuity and compactness of the field-state operator 45

5 Fréchet differentiability of the field-state operator 49

5.1 A general inhomogenous linear Vlasov equation . . . . . . . . . . . . . . . 49

5.2 The Fréchet derivative of the field-state operator . . . . . . . . . . . 55

6 The tracking problem $\quad 61$

6.1 Optimal control with $\mathbb{B}_{K}$-fields . . . . . . . . . . . . . . . 61

6.2 Optimal control by a finite number of field coils . . . . . . . . . . . . . 79

$\begin{array}{lr}\text { Appendix } & 93\end{array}$

Proof of Lemma $15 \ldots \ldots$. . . . . . . . . . . . . . . . . . 93

Proof of Lemma $16 \ldots \ldots \ldots$. . . . . . . . . . . . . . . . 97

Proof of Proposition $24 \ldots \ldots \ldots 10 \ldots$

Proof of Corollary $26 \ldots$. . . . . . . . . . . . . . . . . . . . 104

Proof of Theorem 31 . . . . . . . . . . . . . . . . . . . . . 110

$\begin{array}{ll}\text { Bibliography } & 115\end{array}$ 


\section{Chapter 1}

\section{Introduction}

The three dimensional Vlasov-Poisson system in the plasma physical case is given by the following system of partial differential equations:

$$
\left\{\begin{array}{l}
\partial_{t} f+v \cdot \partial_{x} f-\partial_{x} \psi \cdot \partial_{v} f=0, \\
-\Delta \psi=4 \pi \rho, \quad \lim _{|x| \rightarrow \infty} \psi(t, x)=0 \\
\rho(t, x)=\int f(t, x, v) \mathrm{d} v
\end{array}\right.
$$

Here $f=f(t, x, v) \geq 0$ denotes the distribution function of the particle ensemble that is a scalar function representing the density in phase space. Its time evolution is described by the first line of (1.1) which is a first order partial differential equation referred to as the Vlasov equation. For any measurable set $M \subset \mathbb{R}^{6}$,

$$
\int_{M} f(t, x, v) \mathrm{d}(x, v)
$$

yields the charge of the particles that have space coordinates $x \in \mathbb{R}^{3}$ and velocity coordinates $v \in \mathbb{R}^{3}$ with $(x, v) \in M$ at time $t \geq 0$. The function $\psi$ is the electrostatic potential that is induced by the charge of the particles. It is given by Poisson's equation $-\Delta \psi=4 \pi \rho$ with an homogeneous boundary condition where $\rho$ denotes the volume charge density. The self-consistent electric field is then given by $-\partial_{x} \psi$. Note that both $\psi$ and $-\partial_{x} \psi$ depend linearly on $f$. Hence the Vlasov-Poisson system is nonlinear due to the term $-\partial_{x} \psi \cdot \partial_{v} f$ in the Vlasov equation. Assuming $f$ to be sufficiently regular (e.g., $f(t):=f(t, \cdot, \cdot) \in C_{c}^{1}\left(\mathbb{R}^{6}\right)$ for all $\left.t \geq 0\right)$, we can solve Poisson's equation explicitly and obtain

$$
\psi_{f}(t, x)=\iint \frac{f(t, y, w)}{|x-y|} \mathrm{d} w \mathrm{~d} y \text { for } t \geq 0, x \in \mathbb{R}^{3} .
$$

Considering $f \mapsto \psi_{f}$ to be a linear operator we can formally rewrite the Vlasov-Poisson system as

$$
\partial_{t} f+v \cdot \partial_{x} f-\partial_{x} \psi_{f} \cdot \partial_{v} f=0 .
$$

Combined with the condition

$$
\left.f\right|_{t=0}=\stackrel{\circ}{f}
$$

for some function $\stackrel{\circ}{f} \in C_{c}^{1}\left(\mathbb{R}^{6}\right)$ we obtain an initial value problem. A first local existence and uniqueness result to this initial value problem was proved by Kurth [5]. Later J. Batt [1] established a continuation criterion which claims that a local solution can be extended as long as its velocity support is under control. Finally, two different 
proofs for global existence of classical solutions were established independently and almost simultaneously, one by K. Pfaffelmoser [11] and one by P.-L. Lions and B. Perthame [8]. Later, a greatly simplified version of Pfaffelmoser's proof was published by J. Schaeffer [13]. This means that the follwing result is established: Any nonnegative initial datum $\stackrel{\circ}{f} \in C_{c}^{1}\left(\mathbb{R}^{6}\right)$ launches a global classical solution $f \in C^{1}\left(\left[0, \infty\left[\times \mathbb{R}^{6}\right)\right.\right.$ of the Vlasov-Poisson system (1.1) satisfying the initial condition (1.4). Moreover, for every time $t \in[0, \infty[$, $f(t)=f(t, \cdot, \cdot)$ is compactly supported in $\mathbb{R}^{6}$. Hence equation (1.2) and the reformulation of the Vlasov-Poisson system (1.3) are well-defined in the case $f \in C_{c}^{1}\left(\mathbb{R}^{6}\right)$.

To control the distribution function $f$ we will add an external magnetic field $B$ to the Vlasov equation:

$$
\partial_{t} f+v \cdot \partial_{x} f-\partial_{x} \psi_{f} \cdot \partial_{v} f+(v \times B) \cdot \partial_{v} f=0,\left.\quad f\right|_{t=0}=\stackrel{\circ}{f}
$$

The cross product $v \times B$ occurs since, unlike the electric field, the magnetic field interacts with the particles via Lorentz force. If we want to discuss an optimal control problem where the PDE-constraint is given by (1.5) we must firstly establish the basics for variational calculus. The aims are the following:

- We need some certain set $\mathbb{B}$ such that any field $B \in \mathbb{B}$ induces a unique and sufficiently regular solution $f=f_{B}$ of the initial value problem (1.5).

- The solution $f$ is supposed to exist on any time interval $[0, T]$ which means global existence.

- The solution $f=f_{B}$ is supposed to be continuous and Fréchet differentiable with respect to the field $B$.

- The operator $B \mapsto f_{B}$ is supposed to be weakly compact in some suitable sense.

For fields $B \in C\left([0, T] ; C_{b}^{1}\right)$ we will find out that the Pfaffelmoser-Schaeffer proof can be adapted to this problem. Thus there is a unique classical solution on any time interval $[0, T]$. However, this space is not particularly suitable for optimal control problems where a reflexive Banach space is desired. We will choose the following set to be the set of admissible fields:

$$
\mathbb{B}_{K}:=\left\{B \in L^{2}\left(0, T ; W^{2, \beta} \cap H^{1}\left(\mathbb{R}^{3} ; \mathbb{R}^{3}\right)\right) \mid\|B\|_{L^{2}\left(0, T ; W^{2, \beta}\right)}+\|B\|_{L^{2}\left(0, T ; H^{1}\right)} \leq K\right\}
$$

with $K>0$ and $\beta>3$. Then any field $B \in \mathbb{B}_{K}$ still induces a unique strong solution $f_{B}$ of the initial value problem (1.5) existing on $[0, T]$. It turns out that the high regularity $W^{2, \beta}$ is necessary to provide uniqueness and Fréchet differentiability. Now a field-state operator $\mathbb{B}_{K} \ni B \mapsto f_{B}$ can be defined and we will be able to prove that this operator is Hölder-continuous and Fréchet differentiable. This operator is also weakly compact as any weakly convergent sequence of admissible fields $B_{k} \rightarrow B \in \mathbb{B}_{K}$ yields a sequence of strong solutions $\left(f_{B_{k}}\right)$ with $f_{B_{k}} \rightarrow f_{B}$ in an appropriate sense.

With this foundations it is possible to analyze some application problems. A standard problem is to control $f$ in such a way that it matches a desired distribution function $f_{d}$ at final time $T>0$ as closely as possible. This can, for instance, be modeled by:

$$
\text { Minimize } J(B):=\frac{1}{2}\left\|f_{B}(T)-f_{d}\right\|_{L^{2}}^{2}+\frac{\lambda}{2}\left\|D_{x} B\right\|_{L^{2}\left(0, T ; L^{2}\right)} \quad \text { s.t. } B \in \mathbb{B}_{K} \text {. }
$$


In this model the field $B$ is the control itself but in more realistic models the field will be given by a control-field operator $u \mapsto B(u)$. For example the magnetic field might be generated by $N$ fixed field coils. Each coil generates a magnetic field of a certain shape $m_{i}=m_{i}(x)$ and its intensity is determined by a multiplier $u_{i}=u_{i}(t)$. Then the complete external magnetic field reads as follows:

$$
B(u)(t, x)=\sum_{i=1}^{N} u_{i}(t) m_{i}(t) .
$$

In this case $u=\left(u_{1}, \ldots, u_{N}\right)^{T}$ is the control in this model and we can define a control-field operator $u \mapsto B(u)$.

Both model problems will be analyzed in this paper with respect to the follwing topics:

- existence of a globally optimal solution,

- necessary conditions of first order for locally optimal solutions,

- derivation of an optimality system,

- sufficient conditions of second order for locally optimal solutions,

- uniqueness of the optimal control for small values of $\frac{T}{\lambda}$. 


\section{Chapter 2}

\section{Some important tools}

\subsection{Gronwall's lemma and some generalizations}

Evidently Gronwall's lemma is one of the most important tools in dealing with ordinary differential equations. Especially in this paper, we will require it to analyse the characteristic system of the Vlasov equation. Of course, the "standard version" is well known and is presented only for the sake of completeness. Yet, particularly in the context of $L^{p}$-spaces, we will need some nonlinear generalizations that are also listed in the following lemma.

Lemma 1 Let $I=[a, b]$ be an interval, $A \geq 0$ be any number and let $u, \alpha, \beta, \gamma: I \rightarrow \mathbb{R}_{0}^{+}$ be continuous functions.

(Standard version) Let us assume that the inequality

$$
u(s) \leq \alpha(s)+\int_{a}^{s} \beta(\tau) u(\tau) \mathrm{d} \tau \quad \text { or } \quad u(s) \leq \alpha(s)+\int_{s}^{b} \beta(\tau) u(\tau) \mathrm{d} \tau \text { respectively }
$$

holds for every $s \in[a, b]$. Then for all $s \in[a, b]$,

$$
\begin{aligned}
u(s) & \leq \alpha(s)+\int_{a}^{s} \alpha(\tau) \beta(\tau) \exp \left(\int_{\tau}^{s} \beta(\sigma) \mathrm{d} \sigma\right) \mathrm{d} \tau \\
\text { or } \quad u(s) & \leq \alpha(s)+\int_{s}^{b} \alpha(\tau) \beta(\tau) \exp \left(\int_{s}^{\tau} \beta(\sigma) \mathrm{d} \sigma\right) \mathrm{d} \tau \text { respectively }
\end{aligned}
$$

If additionally $\alpha$ is monotonically increasing or decreasing respectively, then for all $s \in[a, b]$,

$$
u(s) \leq \alpha(s) \exp \left(\int_{a}^{s} \beta(\tau) \mathrm{d} \tau\right) \quad \text { or } \quad u(s) \leq \alpha(s) \exp \left(\int_{s}^{b} \beta(\tau) \mathrm{d} \tau\right) \quad \text { respectively. }
$$

(Quadratic version) Let us assume that the inequality

$$
u(s)^{2} \leq A+\int_{a}^{s} \beta(\tau) u(\tau) \mathrm{d} \tau \quad \text { or } \quad u(s)^{2} \leq A+\int_{s}^{b} \beta(\tau) u(\tau) \mathrm{d} \tau \text { respectively }
$$


holds for every $s \in[a, b]$. Then for all $s \in[a, b]$,

$$
u(s) \leq \sqrt{A}+\frac{1}{2} \int_{a}^{s} \beta(\tau) \mathrm{d} \tau \quad \text { or } \quad u(s) \leq \sqrt{A}+\frac{1}{2} \int_{s}^{b} \beta(\tau) \mathrm{d} \tau \text { respectively. }
$$

( $p$-th power version) Let us assume that the inequality

$$
\begin{gathered}
u(s) \leq A+\int_{a}^{s} \beta(\tau) u(\tau)+\gamma(\tau) u(\tau)^{p} \mathrm{~d} \tau \\
\text { or } u(s) \leq A+\int_{s}^{b} \beta(\tau) u(\tau)+\gamma(\tau) u(\tau)^{p} \mathrm{~d} \tau \text { respectively }
\end{gathered}
$$

holds for every $s \in[a, b]$ and some constant $p \in] 0,1[$. Then for all $s \in[a, b]$,

$$
\begin{aligned}
u(s) \leq & \left\{A^{1-p} \exp \left[(1-p) \int_{a}^{s} \beta(\sigma) \mathrm{d} \sigma\right]\right. \\
& \left.+(1-p) \int_{a}^{s} \gamma(\tau) \exp \left[(1-p) \int_{\tau}^{s} \beta(\sigma) \mathrm{d} \sigma\right] \mathrm{d} \tau\right\}^{\frac{1}{1-p}} \\
\text { or } u(s) \leq & \left\{A^{1-p} \exp \left[(1-p) \int_{a}^{s} \beta(\sigma) \mathrm{d} \sigma\right]\right. \\
& \left.+(1-p) \int_{a}^{s} \gamma(\tau) \exp \left[(1-p) \int_{\tau}^{s} \beta(\sigma) \mathrm{d} \sigma\right] \mathrm{d} \tau\right\}^{\frac{1}{1-p}} \text { respectively. }
\end{aligned}
$$

In the case $\int_{a}^{s}$ the proofs of these inequalities can be found in [2] that is a collection of Gronwall type inequalities by S. Dragomir. Studying these proofs carefully one will easily find out that the case $\int_{s}^{b}$ can be proved completely analogously.

\section{Comment}

(a) When mentioning "Gronwall's lemma" in the following, we refer to the "standard version" from Lemma 1. The other versions will be named explicitely.

(b) The assertions of Lemma 1 hold true if $\beta, \gamma \in L^{2}(] a, b[)$ instead of $\beta, \gamma \in C([a, b])$. We will illustrate this fact by taking the example of the standard version:

If $\beta \in L^{2}(] a, b[)$ there exists some sequence $\left(\beta_{k}\right)_{k \in \mathbb{N}} \subset C([a, b])$ with $\beta_{k} \rightarrow \beta$ in $L^{2}(] a, b[)$. Let $\varepsilon>0$ be arbitrary. As

$$
u(s)<(\alpha(s)+\varepsilon)+\int_{a}^{s} \beta(\tau) u(\tau) \mathrm{d} \tau=\lim _{k \rightarrow \infty}\left((\alpha(s)+\varepsilon)+\int_{a}^{s} \beta_{k}(\tau) u(\tau) \mathrm{d} \tau\right)
$$


we can find $k_{0} \in \mathbb{N}$ such that

$$
u(s) \leq(\alpha(s)+\varepsilon)+\int_{a}^{s} \beta_{k}(\tau) u(\tau) \mathrm{d} \tau
$$

for all $k \geq k_{0}$. Then by Lemma 1 ,

$$
\begin{aligned}
& u(s) \leq(\alpha(s)+\varepsilon)+\int_{a}^{s}(\alpha(\tau)+\varepsilon) \beta_{k}(\tau) \exp \left(\int_{\tau}^{s} \beta_{k}(\sigma) \mathrm{d} \sigma\right) \mathrm{d} \tau \\
& \underset{k \rightarrow \infty}{\rightarrow}(\alpha(\tau)+\varepsilon)+\int_{a}^{s}(\alpha(\tau)+\varepsilon) \beta(\tau) \exp \left(\int_{\tau}^{s} \beta(\sigma) \mathrm{d} \sigma\right) \mathrm{d} \tau \\
& \underset{\varepsilon \rightarrow 0}{\rightarrow} \alpha(s)+\int_{a}^{s} \alpha(\tau) \beta(\tau) \exp \left(\int_{\tau}^{s} \beta(\sigma) \mathrm{d} \sigma\right) \mathrm{d} \tau .
\end{aligned}
$$

Hence

$$
u(s) \leq \alpha(s)+\int_{a}^{s} \alpha(\tau) \beta(\tau) \exp \left(\int_{\tau}^{s} \beta(\sigma) \mathrm{d} \sigma\right) \mathrm{d} \tau
$$

\subsection{A generalization of Jensen's inequality}

Another important tool in the context of $L^{p}$-spaces is Jensen's inequality as it relates the value of a convex/concave function of an integral to the integral of the convex/concave function. In the standard version, the domain of integration must be a set of finite measure. Yet we will now present a generalized version for the domain $\mathbb{R}^{n}$ that has infinite Lebesgue measure.

Lemma 2 Let $n \in \mathbb{N}$ and let $\varphi: \mathbb{R} \rightarrow \mathbb{R}, f: \mathbb{R}^{n} \rightarrow \mathbb{R}$ and $\xi: \mathbb{R}^{n} \rightarrow[1, \infty[$ be Lebesgue measurable functions. We assume that $\int \frac{1}{\xi(x)} \mathrm{d} x=1$. If $\varphi$ is convex it holds that

$$
\varphi\left(\int_{\mathbb{R}^{n}} f(x) \mathrm{d} x\right) \leq \int_{\mathbb{R}^{n}} \varphi(f(x) \xi(x)) \xi(x)^{-1} \mathrm{~d} x
$$

If $\varphi$ is concave the same holds with " $\geq$ " instead of " $\leq$ ".

Proof We define a measure $\mu$ on $\mathbb{R}^{n}$ by $\mathrm{d} \mu(x)=\xi(x)^{-1} \mathrm{~d} x$. This means that for any measurable set $M \subset \mathbb{R}^{n}$,

$$
\mu(M)=\int_{M} \mathrm{~d} \mu(x)=\int_{M} \xi(x)^{-1} \mathrm{~d} x
$$

and especially $\mu\left(\mathbb{R}^{n}\right)=1$. 
Hence if $\varphi$ is convex we have

$$
\begin{aligned}
\varphi\left(\int_{\mathbb{R}^{n}} f(x) \mathrm{d} x\right) & =\varphi\left(\int_{\mathbb{R}^{n}} f(x) \xi(x) \mathrm{d} \mu(x)\right) \leq \int_{\mathbb{R}^{n}} \varphi(f(x) \xi(x)) \mathrm{d} \mu(x) \\
& =\int_{\mathbb{R}^{n}} \varphi(f(x) \xi(x)) \xi(x)^{-1} \mathrm{~d} x
\end{aligned}
$$

by Jensen's inequality. If $\varphi$ is concave Jensen's inequality provides an analogous estimate with " $\geq$ " instead of " $\leq$ ".

\subsection{Sobolev inequalities and continuous embeddings}

Sobolev inequalities or Sobolev's embedding theorems are further important tools we will need in the later approach as they provide continuous embedding of a Sobolev space $W^{k, p}$ either in some $W^{k-1, q}$-space or in some Hölder-Space $C^{l, \gamma}$.

For any open subset $U \subset \mathbb{R}^{n}$ the Hölder space $C^{k, \gamma}(U)$ with $k \in \mathbb{N}$ and $\left.\gamma \in\right] 0,1[$ is defined by

$$
C^{k, \gamma}(U):=\left\{u \in C^{k}(U) \mid\|u\|_{C^{k, \gamma}}<\infty\right\}
$$

where for any $u \in C^{k}(U)$,

$$
\|u\|_{C^{k, \gamma}}:=\max _{|\alpha| \leq k}\left\{\left\|D_{x}^{\alpha} u\right\|_{\infty},\left[D_{x}^{\alpha} u\right]_{\gamma}\right\} \text { with }\left[D_{x}^{\alpha} u\right]_{\gamma}:=\sup _{x \neq y} \frac{\left|D_{x}^{\alpha} u(x)-D_{x}^{\alpha} u(y)\right|}{|x-y|^{\gamma}} .
$$

We will also use the notation $\left[D_{x} u\right]_{\gamma}:=\max _{i=1, \ldots, n}\left[\partial_{x_{i}} u\right]_{\gamma}$. Note that $\left(C^{k, \gamma}(U),\|\cdot\|_{C^{k, \gamma}}\right)$ is a Banach space.

In this paper we will particularly need the following very general version of Sobolev's embedding theorem:

Lemma 3 Let $k \in \mathbb{N}, 1 \leq p<\infty$ and let $U$ be any open subset of $\mathbb{R}^{n}$ with a bounded $C^{1}$-boundary. Moreover let $u \in W^{k, p}(U)$ be arbitrary.

(a) We assume that $k<\frac{n}{p}$ and define $q:=\frac{n p}{n-k p}$, i.e., $\frac{1}{q}=\frac{1}{p}-\frac{k}{n}$. Then $u \in L^{q}(U)$ with $\|u\|_{L^{q}(U)} \leq C\|u\|_{W^{k, p}(U)}$ where $C$ denotes a positive constant that depends only on $k, n, p$ and $U$.

(b) We assume that $k>\frac{n}{p}$. If $\frac{n}{p} \in \mathbb{N}$ we will additionally assume that $U$ is bounded. Then $u \in C^{k-\lfloor n / p\rfloor-1, \gamma}(U)$ where

$$
\gamma= \begin{cases}1+\lfloor n / p\rfloor-n / p, & \text { if } \frac{n}{p} \notin \mathbb{N} \\ \text { is any number in }] 0,1[, & \text { if } \frac{n}{p} \in \mathbb{N}\end{cases}
$$

and

$$
\|u\|_{C^{k-\lfloor n / p\rfloor-1, \gamma}(U)} \leq C\|u\|_{W^{k, p}(U)}
$$

where $C$ denotes a positive constant depending only on $k, n, p, \gamma$ and $U$. 
In the case that $U$ is a bounded subset of $\mathbb{R}^{n}$ with $C^{1}$-boundary a proof is presented by L. C. Evans in [3]. Studying this proof carefully one will find out that the boundedness of $U$ is necessary only in the case $k>\frac{n}{p} \in \mathbb{N}$. If $k<\frac{n}{p}$ or $k>\frac{n}{p} \notin \mathbb{N}$ it suffices to assume that the $C^{1}$-boundary of $U$ is bounded. Note that the whole space $\mathbb{R}^{n}$ satisfies this condition trivially as its boundary is empty.

\subsection{A relation of Sobolev spaces and Bochner spaces}

The identities that are presented in the following lemmata seem to be obvious at first appearance. However, measurability in an $L^{p}$ space and measurability in a Bochner space are two different concepts. Therefore we will give a detailed proof of those assertions. In the following, $C_{c}\left([0, T] \times \mathbb{R}^{n}\right)$ will denote the space of compactly supported continuous functions whose support lies in $[0, T] \times \mathbb{R}^{n}$ but not necessarily in $] 0, T\left[\times \mathbb{R}^{n}\right.$.

Notation For brevity we will sometimes omit the argument " $\left(\mathbb{R}^{n}\right)$ " (for any $n \in \mathbb{N}$ ) when denoting a function space. For instance, we will just write $L^{p}, W^{k, p}$ or $C_{b}^{k}$ instead of $L^{p}\left(\mathbb{R}^{n}\right), W^{k, p}\left(\mathbb{R}^{n}\right)$ or $C_{b}^{k}\left(\mathbb{R}^{n}\right)$. If the function space refers to a proper subset $\Omega \subset \mathbb{R}^{n}$ we will not use this abbreviation.

Lemma 4 Let $1 \leq p<\infty$ and $T>0$ be any real numbers. Then the following holds:

(a) $C_{c}\left([0, T] \times \mathbb{R}^{n}\right)$ is dense in the Bochner space $L^{p}\left(0, T ; L^{p}\left(\mathbb{R}^{n}\right)\right)$,

(b) $L^{p}(] 0, T\left[\times \mathbb{R}^{n}\right)=L^{p}\left(0, T ; L^{p}\left(\mathbb{R}^{n}\right)\right)$.

Proof Step 1: At first we will show that $L^{p}(] 0, T\left[\times \mathbb{R}^{n}\right)$ is a subset of $L^{p}\left(0, T ; L^{p}\left(\mathbb{R}^{n}\right)\right)$. Let $f \in L^{p}(] 0, T\left[\times \mathbb{R}^{n}\right)$ and $g \in L^{q}\left(\mathbb{R}^{n}\right)$ be arbitrary where $q:=\frac{p}{p-1}$ if $p>1$ and $q:=\infty$ if $p=1$ denotes the dual exponent of $p$. Then by Fubini's theorem, the function

$$
] 0, T\left[\ni t \mapsto \int f(t, x) g(x) \mathrm{d} x\right.
$$

is measurable. As $g$ was arbitrary this implies that the function $t \mapsto f(t)$ is weakly measurable in the Banach space $L^{p}\left(\mathbb{R}^{n}\right)$. Then, since $L^{p}\left(\mathbb{R}^{n}\right)$ is separable, we can deduce that $t \mapsto f(t)$ is strongly measurable in $L^{p}\left(\mathbb{R}^{n}\right)$ according to B. J. Pettis [10] and especially $f(t) \in L^{p}\left(\mathbb{R}^{n}\right)$ for almost all $t \in[0, T]$. Thus $t \mapsto\|f(t)\|_{L^{p}}$ is measurable and

$$
\int_{0}^{T}\|f(t)\|_{L^{p}}^{p} \mathrm{~d} t=\|f\|_{L^{p}(] 0, T\left[\times \mathbb{R}^{n}\right)}^{p}<\infty
$$

which means that $f \in L^{p}\left(0, T ; L^{p}\left(\mathbb{R}^{n}\right)\right)$.

Step 2: We will prove that any function $f \in L^{p}\left(0, T ; L^{p}\left(\mathbb{R}^{n}\right)\right)$ can be approximated by a sequence $\left(f_{k}\right)_{k \in \mathbb{N}} \subset C_{c}\left([0, T] \times \mathbb{R}^{n}\right)$. Therefore we consider an arbitrary function $f \in L^{p}\left(0, T ; L^{p}\left(\mathbb{R}^{n}\right)\right)$. According to K. Yosida [15, Chap. V, Sec. 4-5] we can approximate $f$ by a sequence of finitely-valued functions, i.e., for any $k \in \mathbb{N}$, there exist functions $\zeta_{i}^{k} \in L^{p}\left(\mathbb{R}^{n}\right), i=1, \ldots, k$ and a family of pairwise disjoint open subsets $I_{i}^{k} \subset[0, T]$, $i=1, \ldots, k$ with $\lambda\left([0, T] \backslash \bigcup_{i=1}^{k} I_{i}^{k}\right)=0$ such that the sequence defined by 


$$
f_{k}^{(1)}(t, x):=\sum_{i=1}^{k} \mathbb{1}_{I_{i}^{k}}(t) \zeta_{i}^{k}(x), \quad t \in[0, T], x \in \mathbb{R}^{n}
$$

satisfies

$$
\lim _{k \rightarrow \infty}\left\|f_{k}^{(1)}(t)-f(t)\right\|_{L^{p}}=0
$$

for almost every $t \in[0, T]$. Since $C_{c}\left(\mathbb{R}^{n}\right)$ is dense in $L^{p}\left(\mathbb{R}^{n}\right)$, there exist $\xi_{i}^{k} \in C_{c}\left(\mathbb{R}^{n}\right)$, $i=1, \ldots, k$ such that $\left\|\xi_{i}^{k}-\zeta_{i}^{k}\right\|_{L^{p}} \leq \frac{1}{k^{2}}$ for every $i \in\{1, \ldots, k\}$. Hence we have

$$
\lim _{k \rightarrow \infty}\left\|f_{k}^{(2)}(t)-f_{k}^{(1)}(t)\right\|_{L^{p}}=0 \quad \Rightarrow \quad \lim _{k \rightarrow \infty}\left\|f_{k}^{(2)}(t)-f(t)\right\|_{L^{p}}=0
$$

for almost every $t \in[0, T]$ where

$$
f_{k}^{(2)}(t, x):=\sum_{i=1}^{k} \mathbb{1}_{I_{i}^{k}}(t) \xi_{i}^{k}(x), \quad t \in[0, T], x \in \mathbb{R}^{n} .
$$

Now we define

$$
J_{i}^{k}:=\left\{t \in I_{i}^{k} \mid\left\|f_{k}^{(2)}(t)\right\|_{L^{p}} \leq 2\|f(t)\|_{L^{p}}+1\right\} .
$$

For almost every $t \in[0, T]$ there exists $i \in\{1, \ldots, k\}$ such that $t \in I_{i}^{k}$. Assuming $k$ to be sufficiently large, we also have $t \in J_{i}^{k}$. Hence

$$
\lim _{k \rightarrow \infty}\left\|f_{k}^{(3)}(t)-f_{k}^{(2)}(t)\right\|_{L^{p}}=0 \quad \Rightarrow \quad \lim _{k \rightarrow \infty}\left\|f_{k}^{(3)}(t)-f(t)\right\|_{L^{p}}=0
$$

for almost every $t \in[0, T]$ where

$$
f_{k}^{(3)}(t, x):=\sum_{i=1}^{k} \mathbb{1}_{J_{i}^{k}}(t) \xi_{i}^{k}(x), \quad t \in[0, T], x \in \mathbb{R}^{n} .
$$

Since $\left\|f_{k}^{(3)}(t)-f(t)\right\|_{L^{p}} \leq 3\|f(t)\|_{L^{p}}+1$ for every $k \in \mathbb{N}$, Lebesgue's dominated convergence yields

$$
\lim _{k \rightarrow \infty}\left\|f_{k}^{(3)}-f\right\|_{L^{p}\left(0, T ; L^{p}\right)}=0
$$

For $k \in \mathbb{N}$, we choose $\chi_{i}^{k} \in C([0, T]), i=1, \ldots, k$ such that

$$
\left\|\chi_{i}^{k}-\mathbb{1}_{J_{i}^{k}}\right\|_{L^{p}([0, T])}^{p} \leq \frac{1}{k^{3} \max _{1 \leq m \leq k}\left\|\xi_{m}^{k}\right\|_{L^{p}}}, \quad i \in\{1, \ldots, k\}
$$

and define a sequence of $C_{c}\left([0, T] \times \mathbb{R}^{n}\right)$-functions by

$$
f_{k}^{(4)}(t, x):=\sum_{i=1}^{k} \chi_{i}^{k}(t) \xi_{i}^{k}(x), \quad t \in[0, T], x \in \mathbb{R}^{n} .
$$


Then

$$
\begin{aligned}
\left\|f_{k}^{(4)}-f_{k}^{(3)}\right\|_{L^{p}\left(0, T ; L^{p}\right)}^{p} & \leq k^{(p-1) / p} \sum_{i=1}^{k}\left(\int_{0}^{T}\left|\chi_{i}^{k}(t)-\mathbb{1}_{J_{i}^{k}}(t)\right|^{p} \mathrm{~d} t\left\|\xi_{i}^{k}\right\|_{L^{p}}^{p}\right) \\
& \leq \frac{1}{k} \rightarrow 0, \quad k \rightarrow \infty
\end{aligned}
$$

which directly implies that

$$
\lim _{k \rightarrow \infty}\left\|f_{k}^{(4)}-f\right\|_{L^{p}\left(0, T ; L^{p}\right)}=0
$$

Step 3: Now we will show that $L^{p}\left(0, T ; L^{p}\left(\mathbb{R}^{n}\right)\right)$ is a subset of $L^{p}(] 0, T\left[\times \mathbb{R}^{n}\right)$. Therefore let $f \in L^{p}\left(0, T ; L^{p}\left(\mathbb{R}^{n}\right)\right)$ be arbitrary. According to Step 2 there exists some sequence $\left(f_{k}\right) \subset C_{c}\left([0, T] \times \mathbb{R}^{n}\right) \subset L^{p}(] 0, T\left[\times \mathbb{R}^{n}\right)$ such that $f_{k} \rightarrow f$ in $L^{p}\left(0, T ; L^{p}\left(\mathbb{R}^{n}\right)\right)$. This means that $\left(f_{k}\right)$ is a Cauchy sequence in $L^{p}\left(0, T ; L^{p}\left(\mathbb{R}^{n}\right)\right)$, i.e., for all $\varepsilon>0$ there exists $k_{0} \in \mathbb{N}$ such that for all $k, l \in \mathbb{N}$ with $k, l \geq k_{0}$,

$$
\left\|f_{k}-f_{l}\right\|_{L^{p}(] 0, T\left[\times \mathbb{R}^{n}\right)}=\left\|f_{k}-f_{l}\right\|_{L^{p}\left(0, T ; L^{p}\right)}<\varepsilon
$$

Hence $\left(f_{k}\right)$ is also a Cauchy sequence in $L^{p}(] 0, T\left[\times \mathbb{R}^{n}\right)$ and consequently, because of completeness, there exists some function $f^{*} \in L^{p}(] 0, T\left[\times \mathbb{R}^{n}\right)$ such that $f_{k} \rightarrow f^{*}$ in $L^{p}(] 0, T\left[\times \mathbb{R}^{n}\right)$. Then, however, Step 1 yields that $f^{*} \in L^{p}\left(0, T ; L^{p}\left(\mathbb{R}^{n}\right)\right)$ and thus

$$
\left\|f_{k}-f^{*}\right\|_{L^{p}\left(0, T ; L^{p}\right)}=\left\|f_{k}-f^{*}\right\|_{L^{p}(] 0, T\left[\times \mathbb{R}^{n}\right)} \rightarrow 0, \quad k \rightarrow \infty .
$$

As the limit is unique we have $f=f^{*} \in L^{p}(] 0, T\left[\times \mathbb{R}^{n}\right)$.

Lemma 5 Let $1 \leq p<\infty$ and $T>0$ be any real numbers. Then

$$
W^{1, p}(] 0, T\left[\times \mathbb{R}^{n}\right)=W^{1, p}\left(0, T ; L^{p}\left(\mathbb{R}^{n}\right)\right) \cap L^{p}\left(0, T ; W^{1, p}\left(\mathbb{R}^{n}\right)\right) .
$$

Proof In this proof $\partial_{t} f$ and $\partial_{x} f=\left(\partial_{x_{1}} f, \ldots, \partial_{x_{n}} f\right)^{T}$ will denote the partial derivatives of a function $f \in W^{1, p}(] 0, T\left[\times \mathbb{R}^{n}\right)$. On the other hand $\dot{f}$ will denote the derivative of $f \in W^{1, p}\left(0, T ; L^{p}\left(\mathbb{R}^{n}\right)\right)$ and $\nabla f$ will denote the derivative of $f \in L^{p}\left(0, T ; W^{1, p}\left(\mathbb{R}^{n}\right)\right)$. We already know from Lemma 4 that $L^{p}(] 0, T\left[\times \mathbb{R}^{n}\right)=L^{p}\left(0, T ; L^{p}\left(\mathbb{R}^{n}\right)\right)$.

Step 1: At first we will show that $W^{1, p}(] 0, T\left[\times \mathbb{R}^{n}\right)$ is a subset of $W^{1, p}\left(0, T ; L^{p}\left(\mathbb{R}^{n}\right)\right)$ $\cap L^{p}\left(0, T ; W^{1, p}\left(\mathbb{R}^{n}\right)\right)$. Therefore let $f \in W^{1, p}(] 0, T\left[\times \mathbb{R}^{n}\right)$ be arbitrary. From Lemma 4 we can conclude that $f, \partial_{t} f$ and $\partial_{x} f$ are in $L^{p}\left(0, T ; L^{p}\left(\mathbb{R}^{n}\right)\right)$. It remains to show that $f$ is differentiable in the $W^{1, p}\left(0, T ; L^{p}\left(\mathbb{R}^{n}\right)\right)$-sense with $\dot{f}=\partial_{t} f$ and in the $L^{p}\left(0, T ; W^{1, p}\left(\mathbb{R}^{n}\right)\right)$ sense with $\nabla f=\partial_{x} f$. We have

$$
\int_{0}^{T} \int f(t, x) \partial_{t} \phi(t, x) \mathrm{d} x \mathrm{~d} t=-\int_{0}^{T} \int \partial_{t} f(t, x) \phi(t, x) \mathrm{d} x \mathrm{~d} t
$$

for any arbitrary test function $\phi \in C_{c}^{\infty}(] 0, T\left[\times \mathbb{R}^{n}\right)$. Let now $\varphi \in C_{c}^{\infty}(] 0, T[)$ and $\psi \in$ 
$C_{c}^{\infty}\left(\mathbb{R}^{n}\right)$ be arbitrary. Then $\varphi \psi \in C_{c}^{\infty}(] 0, T\left[\times \mathbb{R}^{n}\right)$ and hence

$$
\begin{aligned}
& \iint_{0}^{T} f(t, x) \dot{\varphi}(t) \mathrm{d} t \psi(x) \mathrm{d} x=\int_{0}^{T} \int f(t, x) \partial_{t}[\varphi \psi](t, x) \mathrm{d} x \mathrm{~d} t \\
& \quad=-\int_{0}^{T} \int \partial_{t} f(t, x)[\varphi \psi](t, x) \mathrm{d} x \mathrm{~d} t=-\iint_{0}^{T} \partial_{t} f(t, x) \varphi(t) \mathrm{d} t \psi(x) \mathrm{d} x .
\end{aligned}
$$

Since $\psi$ was arbitrary this means

$$
\int_{0}^{T} f(t, x) \dot{\varphi}(t) \mathrm{d} t=-\int_{0}^{T} \partial_{t} f(t, x) \varphi(t) \mathrm{d} t \quad \text { in } \quad L^{p}\left(\mathbb{R}^{n}\right)
$$

and thus $f \in W^{1, p}\left(0, T ; L^{p}\left(\mathbb{R}^{n}\right)\right)$ where the derivative is given by $\dot{f}=\partial_{t} f$. In the same fashion one can show that $f \in L^{p}\left(0, T ; W^{1, p}\left(\mathbb{R}^{n}\right)\right)$ with $\nabla f=\partial_{x} f$.

Step 2: Now we will prove that $W^{1, p}\left(0, T ; L^{p}\left(\mathbb{R}^{n}\right)\right) \cap L^{p}\left(0, T ; W^{1, p}\left(\mathbb{R}^{n}\right)\right)$ is a subset of $W^{1, p}(] 0, T\left[\times \mathbb{R}^{n}\right)$. Suppose that $f \in W^{1, p}\left(0, T ; L^{p}\left(\mathbb{R}^{n}\right)\right) \cap L^{p}\left(0, T ; W^{1, p}\left(\mathbb{R}^{n}\right)\right)$. This directly implies that $f, \dot{f}$ and $\nabla f$ are in $L^{p}(] 0, T\left[\times \mathbb{R}^{n}\right)$ but we must still show that $f$ is differentiable in the $W^{1, p}(] 0, T\left[\times \mathbb{R}^{n}\right)$-sense with $\partial_{t} f=\dot{f}$ and $\partial_{x} f=\nabla f$. Again, let $\phi \in C_{c}^{\infty}(] 0, T\left[\times \mathbb{R}^{n}\right)$ denote an arbitrary test function. Then there exists some constant $R>0$ such that $\operatorname{supp} \phi(t, \cdot) \subset[-R, R]^{n}$ for all $\left.t \in\right] 0, T[$. For any number $N \in \mathbb{N}$ we can split the cube $[-R, R]^{n}$ into disjoint open subcubes $Q_{i}, i=1, \ldots, N^{n}$, all with edge length $2 R / N$, such that

$$
[-R, R]^{n}=\bigcup_{i=1}^{N^{n}} \bar{Q}_{i}
$$

Let $\bar{x}_{i}$ denote the center of the cube $Q_{i}$ and let $q=\frac{p}{p-1}$ denote the dual exponent to $p$. We define

$$
\left.\phi_{N}(t, x):=\sum_{i=1}^{N^{n}} \varphi_{i}(t) \mathbb{1}_{Q_{i}}(x) \quad \text { where } \quad \varphi_{i}(t):=\phi\left(t, \bar{x}_{i}\right), \quad(t, x) \in\right] 0, T\left[\times \mathbb{R}^{n}, N \in \mathbb{N} .\right.
$$

Now $\left|\phi(t, x)-\phi\left(t, \bar{x}_{i}\right)\right| \leq\left\|D_{x} \phi\right\|_{\infty}\left|x-\bar{x}_{i}\right| \leq \sqrt{n} \frac{2 R}{N}\left\|D_{x} \phi\right\|_{\infty}$ if $\left.(t, x) \in\right] 0, T\left[\times Q_{i}\right.$ and hence

$$
\begin{aligned}
\left\|\phi-\phi_{N}\right\|_{L^{q}(] 0, T\left[\times \mathbb{R}^{n}\right)} & \leq\left(\int_{0}^{T} \int\left(\sum_{i=1}^{N^{n}}\left|\phi(t, x)-\phi\left(t, \bar{x}_{i}\right)\right| \mathbb{1}_{Q_{i}}(x)\right)^{q} \mathrm{~d} x \mathrm{~d} t\right)^{1 / q} \\
& \leq \sqrt{3} \frac{2 R}{N}\left\|D_{x} \phi\right\|_{\infty}\left(\int_{0}^{T} \int\left(\sum_{i=1}^{N^{n}} \mathbb{1}_{Q_{i}}(x)\right)^{q} \mathrm{~d} x \mathrm{~d} t\right)^{1 / q} \\
& \leq \sqrt{3} \frac{2 R}{N}\left\|D_{x} \phi\right\|_{\infty}\left(T(2 R)^{n}\right)^{1 / q} \\
& \rightarrow 0, \quad \text { if } N \rightarrow \infty
\end{aligned}
$$

Note that for any $i \in\left\{1, \ldots, N^{n}\right\}$ the function $\varphi_{i}$ is totally continuously differentiable 
with $\dot{\varphi}_{i}=\frac{\mathrm{d}}{\mathrm{d} t}\left[\phi\left(t, \bar{x}_{i}\right)\right]=\partial_{t} \phi\left(t, \bar{x}_{i}\right)$. Thus $\phi_{N}$ is partially differentiable with respect to $t$, and it holds that

$$
\left.\partial_{t} \phi_{N}=\sum_{i=1}^{N^{n}} \dot{\varphi}_{i} \mathbb{1}_{Q_{i}} \quad \text { on }\right] 0, T\left[\times \mathbb{R}^{n}\right.
$$

in the classical sense. Thus the derivative $\partial_{t} \phi$ can be approximated analogously by the sequence $\partial_{t} \phi_{N}$ and we finally have

$$
\phi=\lim _{N \rightarrow \infty} \sum_{i=1}^{N^{n}} \varphi_{i} \mathbb{1}_{Q_{i}} \quad \text { and } \quad \partial_{t} \phi=\lim _{N \rightarrow \infty} \sum_{i=1}^{N^{n}} \dot{\varphi}_{i} \mathbb{1}_{Q_{i}} \quad \text { in } L^{q}(] 0, T\left[\times \mathbb{R}^{n}\right) .
$$

Now, according to the definition of the $W^{1, p}\left(0, T ; L^{p}\left(\mathbb{R}^{n}\right)\right)$-derivative $\dot{f}$,

$$
\int_{0}^{T} f(t, x) \dot{\varphi}_{i}(t) \mathrm{d} t=-\int_{0}^{T} \dot{f}(t, x) \varphi_{i}(t) \mathrm{d} t \quad \text { in } L^{p}\left(\mathbb{R}^{n}\right), i=1, \ldots, N^{n}
$$

and thus

$$
\begin{aligned}
& \int_{0}^{T} \int f(t, x) \partial_{t} \phi(t, x) \mathrm{d} x \mathrm{~d} t=\lim _{N \rightarrow \infty} \sum_{i=1}^{N^{n}} \iint_{0}^{T} f(t, x) \dot{\varphi}_{i}(t) \mathrm{d} t \mathbb{1}_{Q_{i}}(x) \mathrm{d} x \\
& \quad=-\lim _{N \rightarrow \infty} \sum_{i=1}^{N^{n}} \iint_{0}^{T} \dot{f}(t, x) \varphi_{i}(t) \mathrm{d} t \mathbb{1}_{Q_{i}}(x) \mathrm{d} x=-\int_{0}^{T} \int \dot{f}(t, x) \phi(t, x) \mathrm{d} x \mathrm{~d} t .
\end{aligned}
$$

This means that $f$ is weakly partially differentiable with respect to $t$ and its weak derivative is $\partial_{t} f=\dot{f}$. We can similarly prove that $f$ is weakly partially differentiable with respect to $x$ and the weak derivative is $\partial_{x} f=\nabla f$. Hence $W^{1, p}\left(0, T ; L^{p}\left(\mathbb{R}^{n}\right)\right)$ $\cap L^{p}\left(0, T ; W^{1, p}\left(\mathbb{R}^{n}\right)\right)$ is a subset of $W^{1, p}(] 0, T\left[\times \mathbb{R}^{n}\right)$.

This means equality of both spaces and completes the proof.

\subsection{The Newtonian potential}

If $f$ is a compactly supported continuous function the Newtonian potential yields a weak solution of Poisson's equation $-\Delta u=f$. In this thesis it will be used to describe the electric potential that is generated by the charge of the particles. The following lemma presents some well known properties.

Lemma 6 Let $r>0$ be any real number and let $f \in C_{c}\left(\mathbb{R}^{3}\right)$ with supp $f \subset B_{r}(0)$. Moreover, let $u$ be the Newtonian potential of $f$, i.e.,

$$
u(x)=\int \Phi(x-y) f(y) \mathrm{d} y \quad \text { with } \quad \Phi(x)=\frac{1}{4 \pi|x|} \quad x \in \mathbb{R}^{3}
$$


Then the following holds:

(a) $u \in C_{b}^{1}\left(\mathbb{R}^{3}\right)$ and its gradient is given by

$$
\nabla u(x)=\int \nabla \Phi(x-y) f(y) \mathrm{d} y \quad \text { with } \quad \nabla \Phi(x)=-\frac{x}{4 \pi|x|^{3}}, \quad x \in \mathbb{R}^{3} .
$$

(b) For $|x| \rightarrow \infty$,

$$
u(x)=\mathcal{O}\left(|x|^{-1}\right) \quad \text { and } \quad \nabla u(x)=\mathcal{O}\left(|x|^{-2}\right) .
$$

(c) $u$ is a weak solution of Poisson's equation $-\Delta u=f$, i.e.,

$$
\int \nabla u \cdot \nabla \varphi \mathrm{d} x=\int f \varphi \mathrm{d} x \quad \text { for all } \varphi \in C_{c}^{\infty}\left(\mathbb{R}^{3}\right) .
$$

If even $u \in C^{2}\left(\mathbb{R}^{3}\right)$ the same holds in the sense of classical solutions. Note that $f \in C_{c}^{1}\left(\mathbb{R}^{3}\right)$ suffices to ensure that $u \in C^{2}\left(\mathbb{R}^{3}\right)$.

Proof Initially we will assume that $f \in C_{c}^{1}\left(\mathbb{R}^{3}\right)$. For $\varepsilon>0$ we define

$$
u_{\varepsilon}(x):=\int_{|x-y|>\varepsilon} \Phi(x-y) f(y) \mathrm{d} y=\int_{|y|>\varepsilon} \Phi(y) f(x-y) \mathrm{d} y .
$$

Note that for any $\varepsilon>0, \Phi$ is continuously differentiable on the domain $\left\{y \in \mathbb{R}^{3}:|y|>\varepsilon\right\}$ where the gradient $\nabla \Phi$ is the function that is declared in (2.1). Thus by integration by parts and chain rule,

$$
\begin{aligned}
\nabla u_{\varepsilon}(x) & =\int_{|y|>\varepsilon} \Phi(y) \nabla_{x}[f(x-y)] \mathrm{d} y \\
& =-\int_{|y|>\varepsilon} \Phi(y) \nabla f(x-y) \mathrm{d} y \\
& =\int_{|y|>\varepsilon} \nabla \Phi(y) f(x-y) \mathrm{d} y-\int_{|y|=\varepsilon} \Phi(y) f(x-y) \mathrm{d} S(y) \\
& =\int_{|x-y|>\varepsilon} \nabla \Phi(x-y) f(y) \mathrm{d} y-\int_{|y|=\varepsilon} \Phi(y) f(x-y) \mathrm{d} S(y) .
\end{aligned}
$$

We will now assume that $f \in C_{c}\left(\mathbb{R}^{3}\right)$. Since $C_{c}^{1}\left(\mathbb{R}^{3}\right)$ is dense in $C_{c}\left(\mathbb{R}^{3}\right)$ with respect to the infinity norm, $u_{\varepsilon}$ is also continuously differentiable if $f \in C_{c}\left(\mathbb{R}^{3}\right)$ with

$$
\nabla u_{\varepsilon}(x):=\int_{|x-y|>\varepsilon} \nabla \Phi(x-y) f(y) \mathrm{d} y-\int_{|y|=\varepsilon} \Phi(y) f(x-y) \mathrm{d} S(y) .
$$


Then one can easily show that for $\varepsilon \rightarrow 0$,

$$
u_{\varepsilon}(x) \rightarrow u(x) \quad \text { and } \quad \nabla u_{\varepsilon}(x) \rightarrow \int \nabla \Phi(x-y) f(y) \mathrm{d} y
$$

both uniformely in $x$ since

$$
\left|\int_{|y|=\varepsilon} \Phi(y) f(x-y) \mathrm{d} S(y)\right| \leq \varepsilon\|f\|_{\infty} \rightarrow 0, \quad \varepsilon \rightarrow 0 .
$$

Hence $u \in C^{1}\left(\mathbb{R}^{3}\right)$ and $\nabla u$ is given by (2.1). Now we will assume that $|x|>2 r$. Then

$$
|u(x)| \leq \int_{|y|<r} \frac{1}{4 \pi|| x|-| y||}|f(y)| \mathrm{d} y=|x|^{-1} \int_{|y|<r} \frac{1}{4 \pi\left|1-\frac{|y|}{|x|}\right|}|f(y)| \mathrm{d} y \leq \frac{1}{2 \pi}|x|^{-1}\|f\|_{L^{1}}
$$

and similarly $|\nabla u(x)| \leq \frac{1}{2 \pi}|x|^{-2}\|f\|_{L^{1}}$ that is (b). Moreover this implies that $u$ and $\nabla u$ are bounded on $\mathbb{R}^{3}$ which proves (a).

To prove (c) note that $u$ is a solution of Poisson's equation in the sense of distributions according to E. Lieb and M. Loss [6, sect. 6.21]. Then (a) and integration by parts imply the assertion.

The definition of the Newtonian potential does also make sense if $f \in L^{p}\left(\mathbb{R}^{3}\right)$. The following lemma presents some important regularity properties and inequalities. It is commonly referred to as the Calderón-Zygmund inequality.

Lemma 7 Let $1<p<\infty$ and $f \in L^{p}\left(\mathbb{R}^{3}\right)$ with compact support supp $f \subset B_{r}(0)$ for some radius $r>0$. Moreover let $u$ be the Newtonian potential of $f$ as defined in Lemma 6. Then the following holds:

(a) For any $R>0, u \in W^{2, p}\left(B_{R}(0)\right)$ with $-\Delta u=f$ almost everywhere on $B_{R}(0)$ and there exists some constant $C(p, R, r)>0$ such that

$$
\left\|D^{2} u\right\|_{L^{p}\left(B_{R}(0)\right)} \leq C(p, R, r)\|f\|_{p} .
$$

If additionally $p \geq 3$, there exists some constant $c(R, r)>0$ (that does not depend on $p$ ) such that $C(p, R, r) \leq c(R, r) p$.

(b) If $p \geq \frac{4}{3}$, then $D^{2} u \in L^{p}\left(\mathbb{R}^{3}\right)$. If actually $p \geq 3$, there exists some constant $c^{*}(r)>0$ such that

$$
\left\|D^{2} u\right\|_{L^{p}\left(\mathbb{R}^{3}\right)} \leq c^{*}(r) p\|f\|_{p} .
$$

Comment The conditions " $p \geq 3$ " and " $p \geq \frac{4}{3}$ " are not sharp but sufficient for subsequent utilization. 
Proof A proof of (a) can be found in [4, Chap. 9.4] by D. Gilbarg and N. S. Trudinger. Studying this proof carefully, one will notice that the $p$-dependency of the constant originates from the Marcinkiewicz interpolation theorem that is previously presented in [4, Chap. 9.3]. In fact the constant is given explicitely in the end of the proof of this interpolation theorem. From that we can deduce that for $p \geq 3$ and its dual number $q=q(p)$ satisfying $1 / p+1 / q=1$ our constant is given by

$$
C(p, R, r)=\tilde{c}(R, r)\left(\frac{q}{(q-1)(2-q)}\right)^{\frac{1}{q}}=\tilde{c}(R, r)\left(p \frac{p-1}{p-2}\right)^{1-\frac{1}{p}} \leq 2 \tilde{c}(R, r) p
$$

where $\tilde{c}(R, r)$ denotes some further positive constant. This proves (a) if we define $c(R, r):=2 \tilde{c}(R, r)$.

To prove (b) we will assume without loss of generality that $r>\frac{1}{2}$ and choose $R=2 r$. Let $\kappa>0$ denote some generic constant depending at most on $r$ and let $x \in \mathbb{R}^{3}$ be arbitrary with $|x| \geq R=2 r>1$. Then for all $y \in B_{r}(0)$,

$$
|x-y| \geq|| x|-| y||=|x|\left|1-\frac{|y|}{|x|}\right|>\frac{1}{2}|x| \geq r
$$

and hence for all $i, j \in\{1, \ldots, n\}$,

$$
\left|\partial_{x_{i}} \partial_{x_{j}} u(x)\right| \leq \int_{B_{r}(0)} \frac{\kappa}{|x-y|^{3}}|f(y)| \mathrm{d} y \leq \kappa|x|^{-3}\|f\|_{p} .
$$

Thus if $p \geq \frac{4}{3}$,

$$
\begin{aligned}
& \left\|D^{2} u\right\|_{L^{p}\left(\mathbb{R}^{3} \backslash B_{2 r}(0)\right)}=\left(\sum_{i, j=1}^{3} \int_{|x| \geq 2 r}\left|\partial_{x_{i}} \partial_{x_{j}} u(x)\right|^{p} \mathrm{~d} x\right)^{\frac{1}{p}} \leq \kappa\|f\|_{p}\left(\int_{|x| \geq 2 r}|x|^{-3 p} \mathrm{~d} x\right)^{\frac{1}{p}} \\
& \quad \leq \kappa\|f\|_{p}\left(1+\int_{|x| \geq 1}|x|^{-3 p} \mathrm{~d} x\right)^{\frac{1}{p}} \leq \kappa\|f\|_{p}\left(1+\int_{|x| \geq 1}|x|^{-4} \mathrm{~d} x\right)^{\frac{1}{p}} \\
& \quad \leq \kappa\|f\|_{p}\left(1+\int_{|x| \geq 1}|x|^{-4} \mathrm{~d} x\right) \leq \kappa\|f\|_{p}
\end{aligned}
$$

Finally, if $p \geq 3>\frac{4}{3}$,

$$
\begin{aligned}
\left\|D^{2} u\right\|_{L^{p}\left(\mathbb{R}^{3}\right)} & =\left\|D^{2} u\right\|_{L^{p}\left(B_{2 r}(0)\right)}+\left\|D^{2} u\right\|_{L^{p}\left(\mathbb{R}^{3} \backslash B_{2 r}(0)\right)} \\
& \leq c(2 r) p\|f\|_{p}+\kappa\|f\|_{p} \\
& \leq c^{*}(r) p\|f\|_{p}
\end{aligned}
$$

for some constant $c^{*}(r)>0$. 
Proposition 8 Let $r>0$ be any radius. We define

$$
L_{r}^{2}\left(\mathbb{R}^{3}\right):=\left\{\varphi \in L^{2}\left(\mathbb{R}^{3}\right) \mid \operatorname{supp} \varphi \subset B_{r}(0)\right\} .
$$

For any function $f \in L_{r}^{2}\left(\mathbb{R}^{3}\right)$ let $u_{f}$ denote the Newtonian potential of $f$ as defined in Lemma 6. Then the following holds:

(a) $u_{f} \in H_{\text {loc }}^{2}\left(\mathbb{R}^{3}\right)$ with $-\Delta u_{f}=f$ almost everywhere on $\mathbb{R}^{3}$ and its weak gradient $\nabla u_{f}$ is given by (2.1). Moreover, $u_{f}$ has a continuous representative and is the unique solution of the boundary value problem

$$
-\Delta u_{f}=f \text { a.e. on } \mathbb{R}^{3}, \quad \lim _{|x| \rightarrow \infty} u_{f}(x)=0 .
$$

(b) For $|x| \rightarrow \infty$,

$$
u(x)=\mathcal{O}\left(|x|^{-1}\right), \quad \nabla u(x)=\mathcal{O}\left(|x|^{-2}\right) \quad \text { and } \quad D^{2} u(x)=\mathcal{O}\left(|x|^{-3}\right)
$$

(c) Let $R>0$ be any radius. Then the operator $L_{r}^{2}\left(\mathbb{R}^{3}\right) \ni f \mapsto u_{f} \in H^{2}\left(B_{R}(0)\right)$ is linear and continuous, i.e., there exists some constant $C>0$ depending only on $r$ and $R$ such that $\left\|u_{f}\right\|_{H^{2}\left(B_{R}(0)\right)} \leq C\|f\|_{L^{2}}$ for every $f \in L_{r}^{2}\left(\mathbb{R}^{3}\right)$.

(d) There exists some constant $C>0$ depending only on $r$ such that

$$
\left\|u_{f}\right\|_{L^{\infty}\left(\mathbb{R}^{3}\right)} \leq C\|f\|_{L^{2}} .
$$

If additionally $f \in L^{\infty}\left(\mathbb{R}^{3}\right)$ there exists some constant $C>0$ depending only on $r$ such that

$$
\left\|\nabla u_{f}\right\|_{L^{\infty}} \leq C\|f\|_{L^{\infty}} .
$$

(e) If we additionally assume that $f \in L^{\infty}\left(\mathbb{R}^{3}\right)$ then for any $\left.\gamma \in\right] 0,1\left[, u_{f} \in C^{1, \gamma}\left(\mathbb{R}^{3}\right)\right.$ and thus $\nabla u_{f} \in C^{0, \gamma}\left(\mathbb{R}^{3}\right)$.

Proof Since $f \in L_{r}^{2}\left(\mathbb{R}^{3}\right) \subset L_{l o c}^{1}\left(\mathbb{R}^{3}\right)$ it holds that $u_{f} \in L_{l o c}^{1}\left(\mathbb{R}^{3}\right)$ with $-\Delta u_{f}=f$ in the sense of distributions. Moreover the distributional derivative of $u_{f}$ is a function $\nabla u_{f} \in L_{l o c}^{1}$ that is given by

$$
\nabla u_{f}(x)=\int \nabla \Phi(x-y) f(y) \mathrm{d} y
$$

This result is presented by E. Lieb and M. Loss in [6, sect. 6.21]. On the other hand we know from the Calderon-Zygmund inequality (Lemma 7) that $u_{f} \in H_{l o c}^{2}$ with $-\Delta u_{f}=f$ almost everywhere on $\mathbb{R}^{3}$. This means that the distributional derivative is even the derivative in the weak sense. Hence the weak derivative is given by (2.2) and especially $\nabla u_{f} \in H_{l o c}^{1}$. For any $s>0, u_{f} \in H^{2}\left(B_{s}(0)\right)$ and hence $u_{f} \in C_{b}\left(B_{s}(0)\right)$ according to Sobolev's embedding theorem. As $s$ was arbitrary it also holds that $u \in C\left(\mathbb{R}^{3}\right)$.

Let now $C>0$ denote some generic constant depending only on $r$. Without loss of generality we can assume that $r \geq 1$. If $|x| \geq 2 r$ we have $|x-y| \geq \frac{1}{2}|x| \geq 1$ for all $y \in B_{r}(0)$. 
Hence

$$
\begin{gathered}
\left|u_{f}(x)\right| \leq C \int_{|y|<r} \frac{|f(y)|}{|x-y|} \mathrm{d} y \leq C|x|^{-1}\|f\|_{L^{1}}, \\
\left|\nabla u_{f}(x)\right| \leq C \int_{|y|<r} \frac{|f(y)|}{|x-y|^{2}} \mathrm{~d} y \leq C|x|^{-2}\|f\|_{L^{1}}, \\
\left|D^{2} u_{f}(x)\right| \leq C \int_{|y|<r} \frac{|f(y)|}{|x-y|^{3}} \mathrm{~d} y \leq C|x|^{-3}\|f\|_{L^{1}} .
\end{gathered}
$$

This proves (b) and directly implies that $u_{f}$ satisfies the boundary condition

$$
\lim _{|x| \rightarrow \infty} u_{f}(x)=0
$$

Let us now assume that $\tilde{u} \in H_{\text {loc }}^{2}\left(\mathbb{R}^{3}\right)$ is another solution of the boundary value problem. Then $h:=u-\tilde{u}$ satisfies $\Delta h=0$ especially in the sense of distributions. Hence, by Weyl's lemma (cf. E. Lieb, M. Loss [6, sect.9.3]), h is a harmonic function that also satisfies the boundary condition. This directly yields $h=0$ which means uniqueness and completes the proof of (a).

To prove (c) let $R>0$ be arbitrary and let $C>0$ denote some constant that may depend only on $r$ and $R$. Then

$$
\begin{aligned}
& \left\|u_{f}\right\|_{L^{2}\left(B_{R}(0)\right)}^{2}=\int_{|x| \leq R}\left|u_{f}(x)\right|^{2} \mathrm{~d} x \leq \int_{|x| \leq R}\left(\int_{|y| \leq r}|\Phi(x-y)||f(y)| \mathrm{d} y\right)^{2} \mathrm{~d} x \\
& \quad \leq\|f\|_{L^{2}}^{2} \int_{|x| \leq R} \int_{|y| \leq r}|\Phi(x-y)|^{2} \mathrm{~d} y \mathrm{~d} x=\|f\|_{L^{2}}^{2} \int_{|x| \leq R} \int_{|x-y| \leq r}|\Phi(y)|^{2} \mathrm{~d} y \mathrm{~d} x \\
& \quad \leq\|f\|_{L^{2}}^{2} \int_{|x| \leq R} \int_{|y| \leq R+r}|\Phi(y)|^{2} \mathrm{~d} y \mathrm{~d} x \leq C R^{3}\|f\|_{L^{2}}^{2}\|\Phi\|_{L^{2}\left(B_{R+r}(0)\right)}^{2} \leq C\|f\|_{L^{2}}^{2} .
\end{aligned}
$$

From the Hardy-Littlewood-Sobolev inequality we know that

$$
\left\|\nabla u_{f}\right\|_{L^{2}\left(B_{R}(0)\right)} \leq C\|f\|_{L^{6 / 5}} \leq C\|f\|_{L^{2}}
$$

and Lemma 7 yields $\left\|D^{2} u_{f}\right\|_{L^{2}\left(\mathbb{R}^{3}\right)} \leq C\|f\|_{L^{2}}$ because $2>\frac{4}{3}$. Hence we can deduce that $\left\|u_{f}\right\|_{H^{2}\left(B_{R}(0)\right)} \leq C\|f\|_{L^{2}}$ that is (c).

Now we fix $R=2 r$. If $|x| \leq 2 r$,

$$
\begin{aligned}
\left|u_{f}(x)\right| & \leq\|f\|_{L^{2}}\left(\int_{|y|<r}|\Phi(x-y)|^{2} \mathrm{~d} y\right)^{1 / 2}=\|f\|_{L^{2}}\left(\int_{|x-y|<r}|\Phi(y)|^{2} \mathrm{~d} y\right)^{1 / 2} \\
& \leq\|f\|_{L^{2}}\left(\int_{|y|<3 r}|\Phi(y)|^{2} \mathrm{~d} y\right)^{1 / 2} \leq\|\Phi\|_{L^{2}\left(B_{3 r}(0)\right)}\|f\|_{L^{2}}=C\|f\|_{L^{2}}
\end{aligned}
$$




$$
\begin{aligned}
\left|\nabla u_{f}(x)\right| & \leq\|f\|_{L^{\infty}} \int_{|y|<r}|\nabla \Phi(x-y)| \mathrm{d} y=\|f\|_{L^{\infty}} \int_{|x-y|<r}|\nabla \Phi(y)| \mathrm{d} y \\
& \leq\|f\|_{L^{\infty}} \int_{|y|<3 r}|\Phi(y)| \mathrm{d} y \leq\|\nabla \Phi\|_{L^{1}\left(B_{3 r}(0)\right)}\|f\|_{L^{\infty}}=C\|f\|_{L^{\infty}} .
\end{aligned}
$$

If $|x|>2 r$,

$$
\begin{aligned}
\left|u_{f}(x)\right| & \leq C|x|^{-1}\|f\|_{L^{1}} \leq C R^{-1}\|f\|_{L^{2}} \leq C\|f\|_{L^{2}} \\
\left|\nabla u_{f}(x)\right| & \leq C|x|^{-2}\|f\|_{L^{1}} \leq C R^{-2}\|f\|_{L^{\infty}} \leq C\|f\|_{L^{\infty}} .
\end{aligned}
$$

Note that the constant $C$ depends only on $r$ because of the choice $R=2 r$. This is (d).

To prove (e) let $\gamma \in] 0,1\left[\right.$ be arbitrary. Note that $f \in L^{\infty} \cap L_{r}^{2}\left(\mathbb{R}^{3}\right)$ directly implies that $f \in L^{p}\left(\mathbb{R}^{3}\right)$ for any $1 \leq p \leq \infty$. We choose $p=\frac{3}{1-\gamma}>3$, i.e., $\gamma=1+\left\lfloor\frac{3}{p}\right\rfloor-\frac{3}{p}$, Then the Hardy-Littlewood-Sobolev inequality yields

$$
\left\|u_{f}\right\|_{L^{p}\left(\mathbb{R}^{3}\right)} \leq C\|f\|_{L^{3 p /(2 p+1)}\left(\mathbb{R}^{3}\right)} \quad \text { and } \quad\left\|\nabla u_{f}\right\|_{L^{p}\left(\mathbb{R}^{3}\right)} \leq C\|f\|_{L^{3 p /(p+1)}\left(\mathbb{R}^{3}\right)}
$$

and since $p>3>\frac{4}{3}$ we know from the Calderón-Zygmund inequality (Lemma 7) that $D\left(\nabla u_{f}\right)=D^{2} u_{f} \in L^{p}\left(\mathbb{R}^{3}\right)$. Hence $u_{f} \in W^{2, p}\left(\mathbb{R}^{3}\right)$ and then we can deduce from the Sobolev embedding theorem (Lemma 3 ) that $u_{f} \in C^{1, \gamma}\left(\mathbb{R}^{3}\right)$. 


\section{Chapter 3}

\section{Admissible fields and the field-state operator}

In order to write down the three dimensional Vlasov-Poisson system concisely we will at first define some operators and notations:

For $d \in \mathbb{N}, 1 \leq p \leq \infty$ and $r>0$ let $L_{r}^{p}\left(\mathbb{R}^{d}\right)$ denote the set of functions $\varphi \in L^{p}\left(\mathbb{R}^{d}\right)$ having compact support supp $\varphi \subset B_{r}(0) \subset \mathbb{R}^{d}$. Then the operator

$$
\rho .: L_{r}^{2}\left(\mathbb{R}^{6}\right) \rightarrow L^{2}\left(\mathbb{R}^{3}\right), \varphi \mapsto \rho_{\varphi} \quad \text { with } \quad \rho_{\varphi}(x):=\int \varphi(x, v) \mathrm{d} v, x \in \mathbb{R}^{3}
$$

is linear and bounded. It also holds that $\rho_{\varphi} \in L_{r}^{2}\left(\mathbb{R}^{3}\right)$ for any $\varphi \in L_{r}^{2}\left(\mathbb{R}^{6}\right)$. Let now $R>0$ be an arbitrary radius. Then we know from Proposition 8 that

$$
\psi:: L_{r}^{2}\left(\mathbb{R}^{6}\right) \rightarrow H^{2}\left(B_{R}(0)\right), \varphi \mapsto \psi_{\varphi} \quad \text { with } \quad \psi_{\varphi}(x):=\int \frac{\rho_{\varphi}(y)}{|x-y|} \mathrm{d} y
$$

is a linear and bounded operator and its derivative with respect to $x$, that is

$$
\partial_{x} \psi .: L_{r}^{2}\left(\mathbb{R}^{6}\right) \rightarrow H^{1}\left(B_{R}(0)\right), \varphi \mapsto \partial_{x} \psi_{\varphi} \text { with } \partial_{x} \psi_{\varphi}(x)=-\int \frac{x-y}{|x-y|^{3}} \rho_{\varphi}(y) \mathrm{d} y,
$$

is also linear and bounded. Recall that $\psi_{\varphi}$ is the Newtonian potential of $4 \pi \rho_{\varphi}$ and thus, according to Proposition 8, it is the unique $H_{l o c}^{2}$-solution of Poisson's equation $-\Delta_{x} \psi_{\varphi}=4 \pi \rho_{\varphi}$ with $\psi_{\varphi}(x) \rightarrow 0$ if $|x| \rightarrow \infty$. We will also use the notation $\rho_{f}, \psi_{f}$ and $\partial_{x} \psi_{f}$ for functions $f=f(t, x, v)$ with $t \geq 0, x, v \in \mathbb{R}^{3}$. In this case we will write

$$
\rho_{f}(t, x)=\rho_{f(t)}(x), \quad \psi_{f}(t, x)=\psi_{f(t)}(x), \quad \partial_{x} \psi_{f}(t, x)=\partial_{x} \psi_{f(t)}(x)
$$

for any $t$ and $x$. As already mentioned in the introduction we consider the following initial value problem:

$$
\begin{cases}\partial_{t} f+v \cdot \partial_{x} f-\partial_{x} \psi_{f} \cdot \partial_{v} f+(v \times B) \cdot \partial_{v} f=0 & \text { on }[0, T] \times \mathbb{R}^{6} \\ \left.f\right|_{t=0}=\stackrel{\circ}{f} & \text { on } \mathbb{R}^{6} .\end{cases}
$$

In the following let $T>0$ and $\stackrel{\circ}{f} \in C_{c}^{2}\left(\mathbb{R}^{6} ; \mathbb{R}_{0}^{+}\right)$be arbitrary but fixed. $B=B(t, x)$ is a given external magnetic field and $f=f(t, x, v)$ is the distribution function that is supposed to be controlled. Its electric field $\partial_{x} \psi_{f}=\partial_{x} \psi_{f}(t, x)$ is formally defined as stated above. In the following we will show that the solution $f$ satisfies the required condition $" f(t)=f(t, \cdot, \cdot) \in L_{r}^{2}\left(\mathbb{R}^{6}\right)$ " that ensures $\rho_{f}, \psi_{f}$ and $\partial_{x} \psi_{f}$ to be well defined. Of course 
this is possible only if the magnetic field $B$ is regular enough. The regularity of those fields will be specified in the following section.

\subsection{The set of admissible fields}

We will now introduce the set our magnetic fields will belong to: The set of admissible fields.

Definition 9 Let $K>0$ and $3<\beta<\infty$ be arbitrary fixed constants. We define

$$
\begin{gathered}
\mathcal{V}:=L^{2}\left(0, T ; W^{2, \beta}\left(\mathbb{R}^{3} ; \mathbb{R}^{3}\right)\right) \cap L^{2}\left(0, T ; H^{1}\left(\mathbb{R}^{3} ; \mathbb{R}^{3}\right)\right), \\
\|\cdot\|_{\mathcal{V}}:=\|\cdot\|_{L^{2}\left(0, T ; W^{2, \beta}\right)}+\|\cdot\|_{L^{2}\left(0, T ; H^{1}\right)} \\
\mathbb{B}_{K}:=\left\{B \in L^{2}\left(0, T ; W^{2, \beta}\left(\mathbb{R}^{3} ; \mathbb{R}^{3}\right)\right) \mid\|B\|_{L^{2}\left(0, T ; W^{2, \beta}\right)} \leq K\right\} .
\end{gathered}
$$

$\mathbb{B}_{K}$ is called the set of admissible fields.

Some of its most essential properties are listed in the following lemma.

Lemma 10 The set of admissible fields $\mathbb{B}_{K}$ has the following properties:

(a) $\mathbb{B}_{K}$ is a bounded, convex and closed subset of $\mathcal{V}$.

(b) The space $W^{j, \beta}\left(\mathbb{R}^{3} ; \mathbb{R}^{3}\right)$ is continuously embedded in $C^{j-1, \gamma}\left(\mathbb{R}^{3} ; \mathbb{R}^{3}\right)$ for $j \in \mathbb{N}$ and $\gamma=\gamma(\beta)=1-\frac{3}{\beta}$. Thus there exist constants $k_{0}, k_{1}>0$ depending only on $\beta$ such that for all $B \in \mathbb{B}_{K}$,

$$
\|B(t)\|_{C^{0, \gamma}} \leq k_{0}\|B(t)\|_{W^{1, \beta}}, \quad\|B(t)\|_{C^{1, \gamma}} \leq k_{1}\|B(t)\|_{W^{2, \beta}}
$$

for almost all $t \in[0, T]$. Moreover for any $r>0$ there exist constants $k_{2}, k_{3}>0$ depending only on $\beta$ and $r$ such that for all $B \in \mathbb{B}_{K}$,

$\|B(t)\|_{C^{0, \gamma}\left(B_{r}(0)\right)} \leq k_{2}\|B(t)\|_{W^{1, \beta}\left(B_{r}(0)\right)}, \quad\|B(t)\|_{C^{1, \gamma}\left(B_{r}(0)\right)} \leq k_{3}\|B(t)\|_{W^{2, \beta}\left(B_{r}(0)\right)}$ for almost all $t \in[0, T]$.

(c) The space $L^{2}\left(0, T ; W^{2, \beta}\right)$ is continuously embedded in $L^{2}\left(0, T ; C^{1, \gamma}\right)$. Hence for all $B \in \mathbb{B}_{K}$,

$$
\|B\|_{L^{2}\left(0, T ; C^{1, \gamma}\right)} \leq k_{1}\|B\|_{L^{2}\left(0, T ; W^{2, \beta}\right)} \leq k_{1} K
$$

(d) We define

$$
\mathbb{M}:=\left\{\begin{array}{l|l}
\left.B \in C^{\infty}\left([0, T] \times \mathbb{R}^{3} ; \mathbb{R}^{3}\right)\right) & \begin{array}{l}
\|B\|_{L^{2}\left(0, T ; W^{2, \beta}\right)} \leq 2 K \text { and there exists } m>0: \\
\operatorname{supp} B(t) \subset B_{m}(0) \subset \mathbb{R}^{3} \text { for all } t \in[0, T]
\end{array}
\end{array}\right\} .
$$

Then for any $B \in \mathbb{B}_{K}$, there exists a sequence $\left(B_{k}\right)_{k \in \mathbb{N}} \subset \mathbb{M}$ such that

$$
\left\|B-B_{k}\right\|_{L^{2}\left(0, T ; W^{2, \beta}\right)} \rightarrow 0, \quad k \rightarrow \infty .
$$


(e) $\mathbb{B}_{K} \subset \mathcal{V}$ is weakly compact, i.e., any sequence in $\mathbb{B}_{K}$ contains a subsequence converging weakly in $\mathbb{B}_{K}$ with respect to the $\mathcal{V}$-norm.

Proof (a) is obvious and (b) is a direct consequence of Sobolev's embedding theorem (Lemma 3) and the fact that for all $B \in \mathbb{B}_{K}, B(t) \in W^{2, \beta}$ for almost all $t \in[0, T]$. Then the $k_{1}$-inequality of (b) immediately implies (c). Let now $B \in \mathbb{B}_{K}$ be arbitrary. According to K. Yosida [15, Chap. V, Sec. 4-5] we can approximate $B$ by a sequence of finitely-valued functions in the following sense: For any $k \in \mathbb{N}$, there exist $\zeta_{i}^{k} \in W^{2, \beta}, i=1, \ldots k$ and a family of pairwise disjoint open subsets $I_{i}^{k} \subset[0, T], i=1, \ldots, k$ with $\lambda\left([0, T] \backslash \bigcup_{i=1}^{k} I_{i}^{k}\right)=0$ such that the sequence defined by

$$
B_{k}^{(1)}(t, x):=\sum_{i=1}^{k} \mathbb{1}_{I_{i}^{k}}(t) \zeta_{i}^{k}(x), \quad t \in[0, T], x \in \mathbb{R}^{3}
$$

satisfies

$$
\lim _{k \rightarrow \infty}\left\|B_{k}^{(1)}(t)-B(t)\right\|_{W^{2, \beta}}=0
$$

for almost every $t \in[0, T]$. Since $C_{c}^{\infty}\left(\mathbb{R}^{3}\right)$ is dense in $W^{2, \beta}\left(\mathbb{R}^{3}\right)$, there exist $\xi_{i}^{k} \in C_{c}^{\infty}\left(\mathbb{R}^{3}\right)$, $i=1, \ldots, k$ such that $\left\|\xi_{i}^{k}-\zeta_{i}^{k}\right\|_{W^{2, \beta}} \leq \frac{1}{k^{2}}$ for every $i \in\{1, \ldots, k\}$. Hence we have

$$
\lim _{k \rightarrow \infty}\left\|B_{k}^{(2)}(t)-B_{k}^{(1)}(t)\right\|_{W^{2, \beta}}=0 \Rightarrow \quad \lim _{k \rightarrow \infty}\left\|B_{k}^{(2)}(t)-B(t)\right\|_{W^{2, \beta}}=0
$$

for almost every $t \in[0, T]$ where

$$
B_{k}^{(2)}(t, x):=\sum_{i=1}^{k} \mathbb{1}_{I_{i}^{k}}(t) \xi_{i}^{k}(x), \quad t \in[0, T], x \in \mathbb{R}^{3} .
$$

Now we define

$$
J_{i}^{k}:=\left\{t \in I_{i}^{k} \mid\left\|B_{k}^{(2)}(t)\right\|_{W^{2, \beta}} \leq\|B(t)\|_{W^{2, \beta}}+1\right\} .
$$

For almost every $t \in[0, T]$ there exists $i \in\{1, \ldots, k\}$ such that $t \in I_{i}^{k}$. If now $k$ is sufficiently large, we also have $t \in J_{i}^{k}$. Hence

$$
\lim _{k \rightarrow \infty}\left\|B_{k}^{(3)}(t)-B_{k}^{(2)}(t)\right\|_{W^{2, \beta}}=0 \quad \Rightarrow \quad \lim _{k \rightarrow \infty}\left\|B_{k}^{(3)}(t)-B(t)\right\|_{W^{2, \beta}}=0
$$

for almost every $t \in[0, T]$ where

$$
B_{k}^{(3)}(t, x):=\sum_{i=1}^{k} \mathbb{1}_{J_{i}^{k}}(t) \xi_{i}^{k}(x), \quad t \in[0, T], x \in \mathbb{R}^{3} .
$$

Since $\left\|B_{k}^{(3)}(t)-B(t)\right\|_{W^{2, \beta}} \leq 2\|B(t)\|_{W^{2, \beta}}+1$ for every $k \in \mathbb{N}$, Lebesgue's dominated convergence yields

$$
\lim _{k \rightarrow \infty}\left\|B_{k}^{(3)}-B\right\|_{L^{2}\left(0, T ; W^{2, \beta}\right)}=0
$$


For $k \in \mathbb{N}$, we choose $\chi_{i}^{k} \in C^{\infty}([0, T]), i=1, \ldots, k$ such that

$$
\left\|\chi_{i}^{k}-\mathbb{1}_{J_{i}^{k}}\right\|_{L^{2}([0, T])}^{2} \leq \frac{1}{k^{3} \max _{1 \leq m \leq k}\left\|\xi_{m}^{k}\right\|_{W^{2, \beta}}}, \quad i \in\{1, \ldots, k\}
$$

and define

$$
B_{k}^{(4)}(t, x):=\sum_{i=1}^{k} \chi_{i}^{k}(t) \xi_{i}^{k}(x), \quad t \in[0, T], x \in \mathbb{R}^{3} .
$$

Then

$$
\begin{aligned}
\left\|B_{k}^{(4)}-B_{k}^{(3)}\right\|_{\mathcal{V}}^{2} & \leq k \sum_{i=1}^{k}\left(\int_{0}^{T}\left|\chi_{i}^{k}(t)-\mathbb{1}_{J_{i}^{k}}(t)\right|^{2} \mathrm{~d} t\left\|\xi_{i}^{k}\right\|_{W^{2, \beta}}^{2}\right) \\
& \leq \frac{1}{k} \rightarrow 0, \quad k \rightarrow \infty
\end{aligned}
$$

which directly implies that

$$
\lim _{k \rightarrow \infty}\left\|B_{k}^{(4)}-B\right\|_{L^{2}\left(0, T ; W^{2, \beta}\right)}=0
$$

Without loss of generality it holds that $\left\|B_{k}^{(4)}\right\|_{L^{2}\left(0, T ; W^{2, \beta}\right)} \leq 2 K, k \in \mathbb{N}$ and thus $B_{k}^{(4)} \in \mathbb{M}$ for every $k \in \mathbb{N}$. This completes the proof of (d). As $\mathbb{B}_{K}$ is a bounded subset of $L^{2}\left(0, T ; W^{2, \beta}\right)$ the Banach-Alaoglu theorem implies that any sequence $\left(B_{k}\right) \subset \mathbb{B}_{K}$ contains a subsequence $\left(B_{k}^{*}\right)$ that is converging weakly to some limit $B^{*} \in L^{2}\left(0, T ; W^{2, \beta}\right)$. Since $\left(B_{k}^{*}\right) \subset \mathbb{B}_{K}$ is also bounded in $L^{2}\left(0, T ; H^{1}\right)$ we can extract a subsequence $\left(B_{k}^{* *}\right)$ that is converging weakly to some function $B \in L^{2}\left(0, T ; H^{1}\right)$. Because of uniqueness, $B=B^{*}$. Thus $B \in \mathcal{V}$ and $B_{k} \rightarrow B$ in $\mathcal{V}$. From the weak lower semicontinuity of the $\mathcal{V}$-norm we can easily conclude that $\|B\|_{\mathcal{V}} \leq K$ which proves (e).

\subsection{The characteristic flow of the Vlasov equation}

Since the Vlasov equation is a first-order partial differential equation, it suggests itself to consider the characteristic system. On that point, we will consider a general version of the Vlasov equation,

$$
\partial_{t} f+v \cdot \partial_{x} f+F \cdot \partial_{v} f+v \times G \cdot \partial_{v} f=0,
$$

with given fields $F=F(t, x)$ and $G=G(t, x)$. Then the following holds:

Lemma 11 Let $I \subset \mathbb{R}$ be an interval and let $F, G \in C\left(I \times \mathbb{R}^{3} ; \mathbb{R}^{3}\right)$ be continuously differentiable with respect to $x$ and bounded on $J \times \mathbb{R}^{3}$ for every compact subinterval $J \subset I$. Then for every $t \in I$ and $z=(x, v) \in \mathbb{R}^{6}$ there exists a unique solution $I \ni s \mapsto(X, V)(s, t, x, v)$ of the characteristic system

$$
\dot{x}=v, \quad \dot{v}=F(s, x)+v \times G(s, x)
$$

to the initial condition $(X, V)(t, t, x, v)=(x, v)$. 
The characteristic flow $Z:=(X, V)$ has the following properties:

(a) $Z: I \times I \times \mathbb{R}^{6} \rightarrow \mathbb{R}^{6}$ is continuously differentiable.

(b) For all $s, t \in I$ the mapping $Z(s, t, \cdot): \mathbb{R}^{6} \rightarrow \mathbb{R}^{6}$ is a $C^{1}$-diffeomorphism with inverse $Z(t, s, \cdot)$, and $Z(s, t, \cdot)$ is measure preserving, i.e.,

$$
\operatorname{det} \frac{\partial Z}{\partial z}(s, t, z)=1, \quad s, t \in I, z \in \mathbb{R}^{6} .
$$

Proof (a) is well-known from the standard theory of ordinary differential equations. Because of uniqueness $Z(r, t, Z(t, s, z))=Z(r, s, z)$ and thus $Z(s, t, Z(t, s, z))=z$, i.e., $Z^{-1}(s, t, \cdot)=Z(t, s, \cdot)$. Let us now define

$$
D(s, z):=\left(\begin{array}{c}
v \\
F(s, x)+v \times G(s, x)
\end{array}\right), \quad s \in I, z=(x, v) \in \mathbb{R}^{6} .
$$

Then the characteristic system is given by $\dot{z}=D(s, z)$. Since $D$ is source-free, i.e., $\operatorname{div}_{z} D(s, z)=0$, we obtain

$$
\frac{\mathrm{d}}{\mathrm{d} s} \operatorname{det} \frac{\partial Z}{\partial z}(s, t, z)=\operatorname{div}_{z} D(s, Z(s, t, z)) \operatorname{det} \frac{\partial Z}{\partial z}(s, t, z)=0, \quad s, t \in I, z \in \mathbb{R}^{6}
$$

and thus $\operatorname{det} \frac{\partial Z}{\partial z}(s, t, z)=\operatorname{det} \frac{\partial Z}{\partial z}(t, t, z)=1$ for any $s, t \in I$ and $z \in \mathbb{R}^{6}$.

The relation between the characteristic flow and the solution $f$ of the Vlasov equation (3.5) is described by the following lemma.

Lemma 12 Under the assumptions of Lemma 11 the following holds:

(a) A function $f \in C^{1}\left(I \times \mathbb{R}^{6}\right)$ satifies the Vlasov equation (3.5) iff it is constant along every solution of the characteristic system (11).

(b) Suppose that $0 \in I$. For $\stackrel{f}{f} \in C^{1}\left(\mathbb{R}^{6}\right)$ the function

$$
f(t, z):=\stackrel{\circ}{f}(Z(0, t, z)), \quad t \in I, z \in \mathbb{R}^{6}
$$

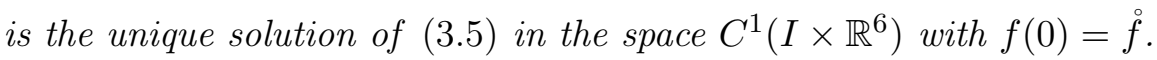

If $\dot{f}$ is nonnegative then so is $f$,

$$
\operatorname{supp} f(t)=Z(t, 0, \operatorname{supp} f), \quad t \in I
$$

and for every $p \in[1, \infty]$,

$$
\|f(t)\|_{p}=\|\stackrel{\circ}{f}\|_{p}, \quad t \in I
$$


Proof For any solution $z=z(s)$ of the characteristic system,

$$
\frac{\mathrm{d}}{\mathrm{d} s} f(s, z(s))=\left(\partial_{t} f+v \cdot \partial_{x} f-\partial_{x} \psi_{f} \cdot \partial_{v} f+v \times B \cdot \partial_{v} f\right)(s, z(s))
$$

This proves (a) since every point $(t, x, v) \in I \times \mathbb{R}^{6}$ is passed through by a characteristic curve. Let now $Z=Z(s, t, z)$ denote the solution of the characteristic system with $Z(t, t, z)=z$. Because of (a), $f$ is a solution of the Vlasov equation iff for all $t \in[0, T]$ and $z \in \mathbb{R}^{6}$,

$$
f(t, z)=f(t, Z(t, t, z))=f(0, Z(0, t, z))=\stackrel{\circ}{f}(Z(0, t, z))
$$

Since $Z$ is uniquely determined and continuously differentiable with respect to all its variables according to Lemma 11 so is $f$. If $1 \leq p<\infty$ we have



by change of variables as the flow is measure preserving. The remaining assertions (including the case $p=\infty$ ) are obvious.

\subsection{Classical solutions for smooth external fields}

As already mentioned in the introduction, the standard initial value problem $((1.3),(1.4))$ posesses a unique global classical solution. This result holds true if the Vlasov equation is equipped with an external magnetic field $B \in C\left([0, T] ; C_{b}^{1}\left(\mathbb{R}^{3} ; \mathbb{R}^{3}\right)\right)$ which will be established in the next theorem. Unfortunately the proof does not work if the field is merely an element of $\mathbb{B}_{K}$. Since such fields are only $L^{2}$ in time, the same holds for the right-hand side of the characteristic system. This makes it impossible to determine a solution in the classical sense of ordinary differential equations. However, we can approximate any field $B \in \mathbb{B}_{K}$ by a sequence $\left(B_{k}\right)_{k \in \mathbb{N}} \subset \mathbb{M} \subset C\left([0, T] ; C_{b}^{1}\left(\mathbb{R}^{3} ; \mathbb{R}^{3}\right)\right)$ according to Lemma 10. This allows us to construct a certain kind of strong solution to the field $B$ as a limit of the classical solutions that are induced by the fields $B_{k}$.

Theorem 13 Let $B \in C\left([0, T] ; C_{b}^{1}\left(\mathbb{R}^{3} ; \mathbb{R}^{3}\right)\right)$ with $\|B\|_{L^{2}\left(0, T ; W^{2, \beta}\right)} \leq 2 K$ be arbitrary. Then there exists a unique classical solution $f \in C^{1}\left([0, T] \times \mathbb{R}^{6}\right)$ of the initial value problem (3.4). Moreover for all $t \in[0, T], f(t)=f(t, \cdot, \cdot)$ is compactly supported in $\mathbb{R}^{6}$ in such a way that there exists some constant $R>0$ depending only on $T, f, K$ and $\beta$ such that for all $t \in[0, T]$,

$$
\operatorname{supp} f(t) \subset B_{R}^{6}(0)=\left\{(x, v) \in \mathbb{R}^{6}:|(x, v)|<R\right\} .
$$

Proof For the standard Vlasov-Poisson system $((1.3),(1.4))$ the existence and uniqueness of a local classical solution was firstly established by R. Kurth [5]. As the field $B \in C\left([0, T] ; C_{b}^{1}\left(\mathbb{R}^{3} ; \mathbb{R}^{3}\right)\right)$ is regular enough the existence and uniqueness of a local classical solution to our problem can be proved analogously. In this thesis we will only sketch the most important steps of that proof. The idea is to define a recursive sequence by

$$
f_{0}(t, z):=\stackrel{\circ}{f}(z) \quad \text { and } \quad f_{k+1}(t, z):=\stackrel{\circ}{f}\left(Z_{k}(0, t, z)\right), \quad k \in \mathbb{N}_{0}
$$


for any $t \geq 0$ and $z=(x, v) \in \mathbb{R}^{6}$ where $Z_{k}$ denotes the solution of

$$
\left(\begin{array}{l}
\dot{x} \\
v
\end{array}\right)=\left(\begin{array}{c}
v \\
-\partial_{x} \psi_{f_{k}}(s, x)+v \times B(s, x)
\end{array}\right) \quad \text { with } \quad Z_{k}(t, t, z)=z .
$$

Then, according to Lemma $12, f_{k+1} \in C^{1}\left([0, T] \times \mathbb{R}^{6}\right)$ is the unique solution of the initial value problem

$$
\partial_{t} f+v \cdot \partial_{x} f-\partial_{x} \psi_{f_{k}} \cdot \partial_{v} f+(v \times B) \cdot \partial_{v} f=0,\left.\quad f\right|_{t=0}=\stackrel{\circ}{f}
$$

We intend to show that the sequence $\left(f_{k}\right)$ converges to the solution of the initial value problem (3.4) if $k$ tends to infinity. By induction we find out that for any $k \in \mathbb{N}_{0}, Z_{k}$ is continuously differentiable with respect to all its variables and hence the same holds for $f_{k}$. One can show that there exists $\delta>0$ and functions $Z$ and $f$ with $Z \in C\left(\left[0, \delta_{0}\right]^{2} \times \mathbb{R}^{6}\right)$, $f \in C\left(\left[0, \delta_{0}\right] \times \mathbb{R}^{6}\right)$ for any $\delta_{0}<\delta$ such that

$$
Z(s, t, z)=\lim _{k \rightarrow \infty} Z_{k}(s, t, z) \quad \text { and } \quad f(t, z)=\stackrel{\circ}{f}(Z(0, t, z))=\lim _{k \rightarrow \infty} f_{k}(t, z)
$$

uniformely in $s, t$ and $z$. For any arbitrary $\delta_{0}<\delta$ it turns out that $\left(\partial_{x} \psi_{f_{k}}\right)$ and $\left(D_{x}^{2} \psi_{f_{k}}\right)$ are Cauchy sequences in $C_{b}\left(\left[0, \delta_{0}\right] \times \mathbb{R}^{3}\right)$. This implies that $\partial_{x} \psi_{f}, D_{x}^{2} \psi_{f} \in C_{b}\left(\left[0, \delta_{0}\right] \times \mathbb{R}^{3}\right)$ and consequently $Z \in C^{1}\left(\left[0, \delta_{0}\right]^{2} \times \mathbb{R}^{6}\right)$. As $\delta_{0}$ was arbitrary this yields $f \in C^{1}\left(\left[0, \delta\left[\times \mathbb{R}^{6}\right)\right.\right.$. Thus $f$ is a local solution of the initial value problem (3.4) on the time interval $[0, \delta[$ according to Lemma 12 as it is constant along any characteristic curve.

Moreover, Batt's continuation criterion (cf. J. Batt [1]) also holds true in our case. We can show that the solution exists on $[0, T]$ by the following argumentation: We assume that $\left[0, T^{*}\left[\right.\right.$ with $T^{*} \leq T$ is the right maximal time interval of the local solution. In this case we will show that

$$
\begin{aligned}
P(t): & =\max \{|v|:(x, v) \in \operatorname{supp} f(s), 0 \leq s \leq t\} \\
& =\max \{|V(s, 0, x, v)|:(x, v) \in \operatorname{supp} \stackrel{\circ}{f}, 0 \leq s \leq t\}
\end{aligned}
$$

is bounded on $\left[0, T^{*}[\right.$. But then, according to Batt, the solution $f$ can be extended beyond $T^{*}$ which is a contradiction as $T^{*}$ was chosen to be maximal. From this we can conclude that the solution exists on the whole time interval $[0, T]$.

For the standard Vlasov-Poisson system (without an external field) such a bound on $P(t)$ is established in the Pfaffelmoser-Schaeffer proof [11, 13]. We will proceed analogously and single out one particle in our distribution. Mathematically, this means to fix a

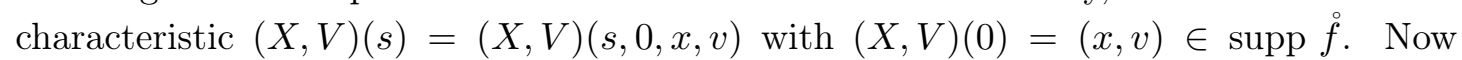
suppose that $0 \leq \delta \leq t<T^{*}$. In the following, constants denoted by $C$ may depend only on $f, T, K$ and $\beta$. The aim in the Pfaffelmoser-Schaeffer proof is to bound the difference $|V(t)-V(t-\delta)|$ from above by an expression in the shape of $C \delta P(t)^{\alpha}$ where $\alpha<1$ is essential. In our case an analogous approach would merely yield some bound that is ideally in the fashion of $C \delta P(t)^{\alpha}+C \sqrt{\delta} P(t)$ because of the additional field term in the $\dot{v}$ equation of the characteristic system. However, we can use the fact that an external magnetic field does not accelerate or slow down the particles. Only the direction of velocity is influenced by $B$ but not its magnitude. This is reflected in the following computation: 
For $s \in[t-\delta, t]$

$$
\begin{aligned}
|V(s)|^{2} & =|V(t-\delta)|^{2}+\int_{t-\delta}^{s} \frac{\mathrm{d}}{\mathrm{d} \tau}|V(\tau)|^{2} \mathrm{~d} \tau \\
& \leq|V(t-\delta)|^{2}+2 \mid \int_{t-\delta}^{s}\left(-\partial_{x} \psi_{f}(\tau, X(\tau))+V(\tau) \times B(\tau, X(\tau)) \cdot V(\tau) \mathrm{d} \tau \mid\right. \\
& \leq P(t-\delta)^{2}+2 \int_{t-\delta}^{s}\left|\partial_{x} \psi_{f}(\tau, X(\tau))\right||V(\tau)| \mathrm{d} \tau .
\end{aligned}
$$

The quadratic version of Gronwall's lemma then implies that

$$
|V(t)| \leq P(t-\delta)+\int_{t-\delta}^{t}\left|\partial_{x} \psi_{f}(s, X(s))\right| \mathrm{d} s \leq P(t-\delta)+\int_{t-\delta}^{t} \iint \frac{f(s, y, w)}{|y-X(s)|^{2}} \mathrm{~d} w \mathrm{~d} y \mathrm{~d} s .
$$

By the change of variables $y=X(s, t, x, v)$ and $w=V(s, t, x, v)$,

$$
|V(t)| \leq P(t-\delta)+\int_{t-\delta}^{t} \iint \frac{f(t, x, v)}{|X(s, t, x, v)-X(s)|^{2}} \mathrm{~d} v \mathrm{~d} x \mathrm{~d} s
$$

since $f$ is constant along the measure perserving characteristic flow. For parameters $0<p \leq P(t)$ and $r>0$, we split the domain of integration in three sets $M_{g}, M_{b}$ and $M_{u}$ which J. Schaeffer called "the Good, the Bad and the Ugly". They are defined as follows:

$$
\begin{aligned}
& M_{g}:=\left\{(s, x, v) \in[t-\delta, t] \times \mathbb{R}^{6}|| v|\leq p \vee| v-V(t) \mid \leq p\right\}, \\
& M_{b}:=\left\{\begin{array}{l|l}
(s, x, v) \in[t-\delta, t] \times \mathbb{R}^{6} \mid \begin{array}{l}
|v|>p \wedge|v-V(t)|>p \\
\wedge\left[\begin{array}{l}
|X(s, t, x, v)-X(s)| \leq r|v|^{-3} \\
\vee \\
\vee X(s, t, x, v)-X(s)|\leq r| v-\left.V(t)\right|^{-3}
\end{array}\right.
\end{array}
\end{array}\right\} \\
& M_{u}:=\left\{\begin{array}{l|l}
(s, x, v) \in[t-\delta, t] \times \mathbb{R}^{6} & \begin{array}{l}
|v|>p \wedge|v-V(t)|>p \\
\wedge|X(s, t, x, v)-X(s)|>r|v|^{-3} \\
\wedge|X(s, t, x, v)-X(s)|>r|v-V(t)|^{-3}
\end{array}
\end{array}\right\}
\end{aligned}
$$

The set $M_{g}$ is the good set since velocities are already bounded, either with respect to our frame of reference or with respect to the particle we singled out. $M_{b}$ is the bad set because velocities are large and the particle whose contribution to the $(x, v)$-integral in (3.8) we are computing is close in space to the singled out particle. This means that the singularity of the Newton force is strong. The set $M_{u}$ is called the ugly set since the integral over this domain cannot be estimated in a straight forward manner although the situation is basically not as bad as in the case of the bad set. To estimate the contribution of each of those sets to the integral in (3.8) the length of the time interval 
is chosen small enough that velocities do not change very much on that interval. The term

$$
\rho_{f}(t, x)=\int f(t, x, v) \mathrm{d} v, \quad t \in\left[0, T^{*}\left[, x \in \mathbb{R}^{3}\right.\right.
$$

can be bounded by $\left\|\rho_{f}(t)\right\|_{\infty} \leq C P(t)^{3}$ for all $t \in\left[0, T^{*}[\right.$. Moreover

$$
\left\|\partial_{x} \psi_{f}(t)\right\|_{\infty} \leq C\left\|\rho_{f}(t)\right\|_{5 / 3}^{5 / 9}\left\|\rho_{f}(t)\right\|_{\infty}^{4 / 9}, \quad t \in\left[0, T^{*}[\right.
$$

according to G. Rein [12, pp.388-390]. In the Pfaffelmoser-Schaeffer proof, one further essential result is that $\left\|\rho_{f}(t)\right\|_{5 / 3}$ is bounded uniformely in $t$ on the time interval $\left[0, T^{*}[\right.$ which is a consequence of energy conservation. Fortunately, the energy is still conserved in our Vlasov equation that is equipped with the magnetic field $B$. Mathematically, this means that

$$
\begin{aligned}
\mathcal{H}(t) & =\frac{1}{2} \iint|v|^{2} f(t) \mathrm{d} v \mathrm{~d} x+\frac{1}{8 \pi} \int\left|\partial_{x} \psi_{f}(t)\right|^{2} \mathrm{~d} x \\
& =\frac{1}{2} \iint|v|^{2} f(t) \mathrm{d} v \mathrm{~d} x+\frac{1}{2} \int \psi_{f}(t) \rho_{f}(t) \mathrm{d} x .
\end{aligned}
$$

does not depend on $t$. By an interpolation argument we obtain that

$$
\left\|\rho_{f}(t)\right\|_{5 / 3}=\left(\int \rho_{f}(t)^{5 / 3} \mathrm{~d} x\right)^{3 / 5} \leq\left(C \iint|v|^{2} f(t) \mathrm{d} v \mathrm{~d} x\right)^{3 / 5} \leq C, \quad t \in\left[0, T^{*}[\right.
$$

as presented by Rein [12, p. 416]. Consequently,

$$
\left\|\partial_{x} \psi_{f}(t)\right\|_{\infty} \leq C^{*} P(t)^{4 / 3}, \quad t \in\left[0, T^{*}[\right.
$$

for some positive constant $C^{*}$ that depends at most on $f$. Now we define

$$
\delta=\delta(t):=\min \left\{1, \frac{t}{2}, \frac{p^{2}}{16\left(C^{*}+2 k_{1} K\right)^{2} P(t)^{8 / 3}}\right\} .
$$

Without loss of generality we may assume that $P(t) \geq 1$ for all $t \in\left[0, T^{*}[\right.$ (otherwise we replace $P(t)$ by $P(t)+1$ ). Thus

$$
\begin{aligned}
|V(s, t, x, v)-v| & =|V(s, t, x, v)-V(t, t, x, v)| \\
& =\int_{s}^{t}|\dot{V}(\tau, t, x, v)| \mathrm{d} \tau \\
& \leq \int_{s}^{t}\left\|\partial_{x} \psi_{f}(\tau)\right\|_{\infty}+P(t)\|B(\tau)\|_{\infty} \mathrm{d} \tau \leq \delta C^{*} P(t)^{4 / 3}+\sqrt{\delta} 2 k_{1} K P(t) \\
& \leq \sqrt{\delta}\left(C^{*}+2 k_{1} K\right) P(t)^{4 / 3} \leq \frac{p}{4}
\end{aligned}
$$

for all $s \in[t-\delta, t]$ and $(x, v) \in \mathbb{R}^{6}$. Now the integrals over the three domains can be estimated just as it is done in the Pfaffelmoser-Schaeffer proof. 
For the sake of brevity we will not present the entire computation but only cite the results:

$$
\begin{aligned}
& \int_{M_{g}} \frac{f(t, x, v)}{|X(s, t, x, v)-X(s)|^{2}} \mathrm{~d}(s, x, v) \leq C \delta p^{4 / 3}, \\
& \int_{M_{b}} \frac{f(t, x, v)}{|X(s, t, x, v)-X(s)|^{2}} \mathrm{~d}(s, x, v) \leq C \delta r \ln \left(\frac{4 P(t)}{p}\right), \\
& \int_{M_{u}} \frac{f(t, x, v)}{|X(s, t, x, v)-X(s)|^{2}} \mathrm{~d}(s, x, v) \leq C r^{-1} .
\end{aligned}
$$

For a detailed derivation of those inequalities confer Rein [12, pp. 418-422]. Thus inequality (3.8) yields

$$
|V(t)| \leq P(t-\delta)+C \delta\left(p^{4 / 3}+r \ln \left(\frac{4 P(t)}{p}\right)+\frac{1}{r \delta}\right) .
$$

We will now choose $p=P(t)^{4 / 7}$ and $r=P(t)^{16 / 21}$ in order to enforce that the terms of the sum on the right-hand side of this estimate are of the same order in $P(t)$. Then

$$
\delta(t)=\min \left\{1, \frac{t}{2}, \frac{1}{16\left(C^{*}+2 k_{1} K\right)^{2}} P(t)^{-32 / 21}\right\} .
$$

Moreover, suppose that

$$
P(t) \geq 1+\left(16\left(C^{*}+2 k_{1} K\right)^{2}\right)^{-21 / 32}, \quad t \in\left[0, T^{*}[\right.
$$

(otherwise we replace $P(t)$ by $\left.P(t)+1+\left(16\left(C^{*}+2 k_{1} K\right)^{2}\right)^{-21 / 32}\right)$. This yields

$$
\delta(t)=\min \left\{\frac{t}{2}, \frac{1}{16\left(C^{*}+2 k_{1} K\right)^{2}} P(t)^{-32 / 21}\right\} .
$$

If for all $t \in\left[0, T^{*}[\right.$,

$$
\frac{1}{16\left(C^{*}+2 k_{1} K\right)^{2}} P(t)^{-32 / 21}>\frac{t}{2}
$$

then immediately $P(t) \leq C^{\prime}$ on $\left[0, T^{*}\right.$ [ for some constant $C^{\prime}>0$ depending only on $\stackrel{f}{\text {, }}$ $K$ and $\beta$. Else there exists

$$
T^{\prime}:=\inf \left\{t \in \left[0, T^{*}\left[\mid \frac{1}{16\left(C^{*}+2 k_{1} K\right)^{2}} P(t)^{-32 / 21} \leq \frac{t}{2}\right\} .\right.\right.
$$

Since $P(t)$ is monotonically increasing,

$$
\delta(t)=\frac{1}{16\left(C^{*}+2 k_{1} K\right)^{2}} P(t)^{-32 / 21}, \quad t \geq T^{\prime} .
$$

Hence $\delta$ is decreasing on $\left[T^{\prime}, T^{*}\left[\right.\right.$. For $t \in\left[T^{\prime}, T^{*}[\right.$,

$$
|V(t)| \leq P(t-\delta(t))+C \delta(t)\left(p^{4 / 3}+r \ln \left(\frac{4 P(t)}{p}\right)+\frac{1}{r} P(t)^{32 / 21}\right)
$$




$$
\begin{aligned}
& \leq P(t-\delta(t))+C \delta(t) P(t)^{16 / 21} \ln (P(t)) \\
& \leq P(t-\delta(t))+C \delta(t) P(t)^{17 / 21} .
\end{aligned}
$$

Let now $\left.t_{0} \in\right] T^{\prime}, T^{*}\left[\right.$ be arbitrary. We define $t_{i+1}:=t_{i}-\delta\left(t_{i}\right)$ as long as $t_{i} \geq T^{\prime}$. Since $t_{i}-t_{i+1}=\delta\left(t_{i}\right) \geq \delta\left(t_{0}\right)$, there exists $k \in \mathbb{N}$ such that

$$
t_{k+1} \leq T^{\prime}<t_{k}<\cdots<t_{0}
$$

Without loss of generality, $t_{k+1}=T^{\prime}$ (otherwise we shrink $\delta\left(t_{k}\right)$ appropriately). Then for $i \in\{1, \ldots, k\}$ and $t \in\left[t_{i+1}, t_{i}\right]$,

$$
|V(t)| \leq P\left(t_{i+1}\right)+C \delta\left(t_{i+1}\right) P\left(t_{0}\right)^{17 / 21} .
$$

Additionally, $|V(t)| \leq P\left(t_{i+1}\right)$ if $t<t_{i+1}$ for any $i \in\{1, \ldots, k\}$. Thus

$$
\begin{aligned}
& P\left(t_{i}\right) \leq P\left(t_{i+1}\right)+C \delta\left(t_{i+1}\right) P\left(t_{0}\right)^{19 / 21}, \\
\text { i.e., } \quad & P\left(t_{i}\right)-P\left(t_{i+1}\right) \leq C \delta\left(t_{i+1}\right) P\left(t_{0}\right)^{19 / 21} .
\end{aligned}
$$

Consequently

$$
P\left(t_{0}\right)-P\left(T^{\prime}\right)=\sum_{i=0}^{k}\left(P\left(t_{i}\right)-P\left(t_{i+1}\right)\right) \leq C P\left(t_{0}\right)^{19 / 21} \sum_{i=0}^{k} \delta\left(t_{i+1}\right) \leq C t_{0} P\left(t_{0}\right)^{19 / 21}
$$

Since $t_{0} \in\left[T^{\prime}, T^{*}\left[\right.\right.$ was arbitrary, this means that for all $t \in\left[T^{\prime}, T^{*}[\right.$,

$$
P(t) \leq P\left(T^{\prime}\right)+C t P(t)^{19 / 21} \leq\left(P(T)^{2 / 21}+C t\right) P(t)^{19 / 21} \leq C^{\prime \prime}(1+t) P(t)^{19 / 21}
$$

where $C^{\prime \prime}$ depends only on $\stackrel{\circ}{f}, K, \beta$ and $T$. This finally yields

$$
P(t) \leq \max \left\{C^{\prime}, C^{\prime \prime}\right\}(1+t)^{21 / 2}, \quad t \in\left[0, T^{*}[.\right.
$$

Now according to the continuation criterion the solution can be extended beyond $T^{*}$ which is a contradiction since $T^{*}$ was chosen as large as possible. This implies that the solution exists on the whole time interval $[0, T]$, and

$$
P(t) \leq C(1+T)^{21 / 2}=: C_{P}, \quad t \in[0, T]
$$

where $C_{P}>0$ is depending only on $T, \stackrel{\circ}{f}, K$ and $\beta$. We will now consider

$$
\begin{aligned}
Q(t): & =\max \{|x|:(x, v) \in \operatorname{supp} f(s), 0 \leq s \leq t\} \\
& =\max \{|X(s, 0, x, v)|:(x, v) \in \operatorname{supp} \stackrel{\circ}{f}, 0 \leq s \leq t\}, \quad t \in[0, T] .
\end{aligned}
$$

Obviously

$$
Q(t) \leq Q(0)+\int_{0}^{T} P(\tau) \mathrm{d} \tau \leq C+T C_{P}=: C_{Q}
$$


so $C_{Q}>0$ also depends only on $T, \stackrel{\circ}{f}, K$ and $\beta$. Finally we define

$$
S(t):=\max \{|(x, v)|:(x, v) \in \operatorname{supp} f(s), 0 \leq s \leq t\}, \quad t \in[0, T]
$$

and obtain

$$
S(t) \leq P(t)+Q(t)<C_{P}+C_{Q}+1=: R, \quad t \in[0, T]
$$

which means that supp $f(t) \subset B_{R}^{6}(0)$ for all $t \in[0, T]$. The proof is complete.

Temporarily we will write $f_{B}$ to denote the classical solution that is induced by the field $B$.

Corollary 14 Suppose that $\stackrel{\circ}{f} \in C_{c}^{2}\left(\mathbb{R}^{6}\right), B \in C\left([0, T] ; C_{b}^{2}\left(\mathbb{R}^{3} ; \mathbb{R}^{3}\right)\right)$ and let $f_{B}$ be the induced classical solution that is given by Theorem 13. Then it additionally holds that $f_{B} \in C\left([0, T] ; C_{b}^{2}\left(\mathbb{R}^{6}\right)\right)$.

For the standard Vlasov-Poisson system (i.e., $B=0$ ) the proof of this assertion is given by $\mathrm{A}$. Lindner in [7]. If $B \in C\left([0, T] ; C_{b}^{2}\left(\mathbb{R}^{3} ; \mathbb{R}^{3}\right)\right)$ is arbitrary the proof proceeds analogously.

In order to prove that any field $B \in \mathbb{B}_{K}$ still induces a strong solution of the initial value problem the following two lemmata are essential. For fields $B \in \mathbb{M}$ Lemma 16 asserts that $f_{B}$ depends Lipschitz continuously on $B$ while its derivatives $\partial_{z} f_{B}$ and $\partial_{t} f_{B}$ are Hölder continuous with respect to $B$. In the course of the construction of a strong solution to some field $B \in \mathbb{B}_{K}$ we will approximate $B$ by a sequence $\left(B_{k}\right) \subset \mathbb{M}$ and then Lemma 16 will ensure that $\left(f_{B_{k}}\right),\left(\partial_{t} f_{B_{k}}\right)$ and $\left(\partial_{z} f_{B_{k}}\right)$ are Cauchy sequences in some sense. To prove Lemma 16 we will need some uniform bounds that are established in Lemma 15.

Lemma 15 Let $B \in \mathbb{M}$ be any admissible field. For $t, s \in[0, T]$ and $z=(x, v) \in \mathbb{R}^{6}$, let $Z_{B}=Z_{B}(s, t, z)=\left(X_{B}, V_{B}\right)(s, t, x, v)$ be the solution of the characteristic system with $Z_{B}(t, t, z)=z$. Furthermore let $f_{B}$ be the classical solution of the initial value problem (3.4) to the field $B$. Then, there exist constants $R_{Z} \geq R, c_{1}, c_{2}, c_{3}, c_{4}>0$ depending only on $f, T, K$, and $\beta$ such that for all $t, s \in[0, T]$,

$$
\begin{array}{ll}
\left\|Z_{B}(s, t, \cdot)\right\|_{L^{\infty}\left(B_{R}^{6}(0)\right)} \leq R_{Z}, & \left\|D_{z} Z_{B}(s, t, \cdot)\right\|_{L^{\infty}\left(B_{R}^{6}(0)\right)} \leq c_{1}, \\
\left\|\partial_{z} f_{B}(t)\right\|_{\infty} \leq c_{2}, & \left\|D_{x}^{2} \psi_{f_{B}}(t)\right\|_{\infty} \leq c_{3}, \quad\left\|\partial_{t} f_{B}\right\|_{L^{2}\left(0, T ; C_{b}\right)} \leq c_{4} .
\end{array}
$$

Moreover $Z_{B}$ and $f_{B}$ are even twice weakly differentiable with respect to $z$ and there exist constants $c_{5}, c_{6}>0$ depending only on $\stackrel{\circ}{f}, T, K$, and $\beta$ such that

$\left\|\left[t \mapsto Z_{B}(s, t, \cdot)\right]\right\|_{L^{\infty}\left(0, T ; W^{2, \beta}\left(B_{R}(0)\right)\right)} \leq c_{5}, \quad s \in[0, T] \quad$ and $\quad\left\|D_{z}^{2} f_{B}\right\|_{L^{\infty}\left(0, T ; L^{\beta}\right)} \leq c_{6}$. 
Lemma 16 Let $B, H \in \mathbb{M}$ be admissible fields and let $f_{B}, f_{H}$ be the induced classical solutions. Moreover, let $Z_{B}$ denote the solution of the characteristic system to the field $B$ satisfying $Z_{B}(t, t, z)=z$ and let $Z_{H}$ be defined analogously. Then, there exist constants $\ell_{1}, \ell_{2}, L_{1}, L_{2}, L_{3}>0$ depending only on $f, T, K$ and $\beta$ such that

$$
\begin{aligned}
\left\|Z_{B}-Z_{H}\right\|_{C\left([0, T] ; C_{b}\left(B_{R}(0)\right)\right)} & \leq \ell_{1}\|B-H\|_{L^{2}\left(0, T ; W^{1, \beta}\left(B_{R_{Z}}(0)\right)\right)}, \\
\left\|\partial_{z} Z_{B}-\partial_{z} Z_{H}\right\|_{C\left([0, T] ; C_{b}\left(B_{R}(0)\right)\right)} & \leq \ell_{2}\|B-H\|_{L^{2}\left(0, T ; W^{2, \beta}\left(B_{R_{Z}}(0)\right)\right)}^{\gamma}, \\
\left\|f_{B}-f_{H}\right\|_{C\left([0, T] ; C_{b}\right)} & \leq L_{1}\|B-H\|_{L^{2}\left(0, T ; W^{1, \beta}\left(B_{R_{Z}}(0)\right)\right)}, \\
\left\|\partial_{z} f_{B}-\partial_{z} f_{H}\right\|_{C\left([0, T] ; C_{b}\right)} & \leq L_{2}\|B-H\|_{L^{2}\left(0, T ; W^{2, \beta}\left(B_{R_{Z}}(0)\right)\right)}^{\gamma}, \\
\left\|\partial_{t} f_{B}-\partial_{t} f_{H}\right\|_{L^{2}\left(0, T ; C_{b}\right)} & \leq L_{3}\|B-H\|_{L^{2}\left(0, T ; W^{2, \beta}\left(B_{R_{Z}}(0)\right)\right)}^{\gamma}
\end{aligned}
$$

where $\gamma=\gamma(\beta)$ is the Hölder exponent from Lemma 10.

The proofs of Lemma 15 and Lemma 16 are very technical and require several Gronwall loops. So as not to disturb the flow of reading, detailed versions of both proofs are outsourced to the Appendix.

\subsection{Strong solutions for admissible external fields}

Now we will show that any field $B \in \mathbb{B}_{K}$ still induces a unique strong solution which can be constructed as the limit of solutions $f_{B_{k}}$ where $\left(B_{k}\right) \subset \mathbb{M}$ with $B_{k} \rightarrow B$ in $L^{2}\left(0, T ; W^{2, \beta}\right)$. Such a strong solution is defined as follows:

Definition 17 Let $B \in \mathbb{B}_{K}$ be any admissible field. We call $f$ a strong solution of the initial value problem (3.4) to the field $B$, iff the following holds:

(i) For all $1 \leq p \leq \infty, f \in W^{1,2}\left(0, T ; L^{p}\left(\mathbb{R}^{6}\right)\right) \cap L^{2}\left(0, T ; W^{1, p}\left(\mathbb{R}^{6}\right)\right) \subset C\left([0, T] ; L^{p}\left(\mathbb{R}^{6}\right)\right)$ and

$$
\|f\|_{W^{1,2}\left(0, T ; L^{p}\right)}+\|f\|_{L^{2}\left(0, T ; W^{1, p}\right)} \leq C
$$

for some constant $C>0$ depending only on $\stackrel{\circ}{f}, T, K$ and $\beta$.

(ii) $f$ satisfies the Vlasov equation

$$
\partial_{t} f+v \cdot \partial_{x} f-\partial_{x} \psi_{f} \cdot \partial_{v} f+(v \times B) \cdot \partial_{v} f=0
$$

almost everywhere on $[0, T] \times \mathbb{R}^{6}$.

(iii) $f$ satisfies the initial condition $\left.f\right|_{t=0}=\stackrel{\circ}{f}$ almost everywhere on $\mathbb{R}^{6}$,

(iv) For every $t \in[0, T]$, supp $f(t) \subset B_{R}(0)$ where $R$ is the constant from Theorem 13 .

First of all one can easily establish that such a strong solution is unique.

Proposition 18 Let $B \in \mathbb{B}_{K}$ be any field and suppose that there exists a strong solution $f$ of the initial value problem (3.4) to the field B. Then this solution is unique. 
Proof Suppose that there exists another strong solution $g$ to the field $B$. Then the difference $h:=f-g$ satisfies

$$
\partial_{t} h+v \cdot \partial_{x} h-\partial_{x} \psi_{h} \cdot \partial_{v} f-\partial_{x} \psi_{g} \cdot \partial_{v} h+(v \times B) \cdot \partial_{v} h=0 \text {. }
$$

almost everywhere on $[0, T] \times \mathbb{R}^{6}$. Thus by integration by parts and Proposition 8 ,

$$
\begin{aligned}
\frac{\mathrm{d}}{\mathrm{d} t}\|h(t)\|_{L^{2}}^{2} & =2 \int \partial_{t} h h \mathrm{~d} z=2 \int \partial_{x} \psi_{h} \cdot \partial_{v} f h \mathrm{~d} z \leq 2\left\|\partial_{v} f(t)\right\|_{\infty}\left\|\partial_{x} \psi_{h}(t)\right\|_{L^{2}}\|h(t)\|_{L^{2}} \\
& \leq C(R)\left\|\partial_{v} f(t)\right\|_{\infty}\|h(t)\|_{L^{2}}^{2} .
\end{aligned}
$$

As $t \mapsto\|h(t)\|_{L^{2}}^{2}$ is continuous with $\|h(0)\|_{L^{2}}^{2}=0$, Gronwall's lemma yields $\|h(t)\|_{L^{2}}^{2}=0$ for all $t \in[0, T]$. Hence for all $t \in[0, T], f(t)=g(t)$ almost everywhere on $\mathbb{R}^{6}$ which means uniqueness.

Now we will show that any admissible field $B \in \mathbb{B}_{K}$ actually induces a unique strong solution. Note that this solution is even more regular than it was demanded in the definition. However the weaker requirements of the definition will be essential in the later approach (see Proposition 22) and it will also be important that uniqueness was established under those weaker conditions.

Theorem 19 Let $B \in \mathbb{B}_{K}$. Then there exists a unique strong solution $f$ of the initial value problem (3.4) to the field B. Moreover this solution satisfies the following properties which are even stronger than the conditions that are demanded in Definition 1\%:

(a) $f \in W^{1,2}\left(0, T ; C_{b}\left(\mathbb{R}^{6}\right)\right) \cap C\left([0, T] ; C_{b}^{1}\left(\mathbb{R}^{6}\right)\right) \cap L^{\infty}\left(0, T ; W^{2, \beta}\left(\mathbb{R}^{6}\right)\right)$ with

$$
\|f(t)\|_{p}=\|\stackrel{\circ}{f}\|_{p}, \quad t \in[0, T], \quad 1 \leq p \leq \infty
$$

and

$$
\|f\|_{W^{1,2}\left(0, T ; C_{b}\right)}+\|f\|_{C\left([0, T] ; C_{b}^{1}\right)}+\|f\|_{L^{\infty}\left(0, T ; W^{2, \beta}\right)} \leq C
$$

for some constant $C>0$ depending only on $\stackrel{\circ}{f} T, K$ and $\beta$.

(b) $f$ satisfies the initial condition $\left.f\right|_{t=0}=\stackrel{\circ}{f}$ everywhere on $\mathbb{R}^{6}$,

Proof Let $B \in \mathbb{B}_{K}$ arbitrary. According to Lemma 10, we can choose some sequence $\left(B_{k}\right)_{k \in \mathbb{N}} \subset \mathbb{M}$ with $B_{k} \rightarrow B$ for $k \rightarrow \infty$ in $L^{2}\left(0, T, W^{2, \beta}\right)$. Now Lemma 16 and Lemma 15 provide that for all $t \in[0, T]$ and $j, k \in \mathbb{N}$,

$$
\begin{aligned}
&\left\|f_{B_{k}}-f_{B_{j}}\right\|_{C\left([0, T] ; C_{b}\right)} \leq L_{1}\left\|B_{k}-B_{j}\right\|_{L^{2}\left(0, T ; W^{2, \beta}\left(B_{R_{Z}}(0)\right)\right)}, \\
&\left\|\partial_{z} f_{B_{k}}-\partial_{z} f_{B_{j}}\right\|_{C\left([0, T] ; C_{b}\right)} \leq L_{3}\left\|B_{k}-B_{j}\right\|_{L^{2}\left(0, T ; W^{2, \beta}\left(B_{R_{Z}}(0)\right)\right)}^{\gamma}, \\
&\left\|\partial_{t} f_{B_{k}}-\partial_{t} f_{B_{j}}\right\|_{L^{2}\left(0, T ; C_{b}\right)} \leq L_{4}\left\|B_{k}-B_{j}\right\|_{L^{2}\left(0, T ; W^{2, \beta}\left(B_{R_{Z}}(0)\right)\right)}^{\gamma}, \\
&\left\|D_{z}^{2} f_{B_{k}}\right\|_{L^{\infty}\left(0, T ; L^{\beta}\right)} \leq c_{6} .
\end{aligned}
$$

where $\gamma=\gamma(\beta)$ is the constant from Lemma 10. Hence, $\left(f_{B_{k}}\right)_{n \in \mathbb{N}}$ is a Cauchy sequence in $C\left([0, T] ; C_{b}^{1}\right) \cap W^{1,2}\left(0, T ; C_{b}\right)$. Due to completeness there exists a unique function $f \in C\left([0, T] ; C_{b}^{1}\right) \cap W^{1,2}\left(0, T ; C_{b}\right)$ such that $f_{B_{k}} \rightarrow f$ in $C\left(\left[0, T\left[; C_{b}^{1}\right) \cap W^{1,2}\left(0, T ; C_{b}\right)\right.\right.$. 
Since $\left(f_{B_{k}}\right)$ is also bounded in $L^{\infty}\left(0, T ; W^{2, \beta}\right)$ by some constant depending only on $f, T, K$ and $\beta$, the Banach-Alaoglu theorem states that there exists some function $\bar{f} \in L^{\infty}\left(0, T ; W^{2, \beta}\right)$ such that $f_{B_{k}} \stackrel{*}{\rightarrow} \bar{f}$ up to a subsequence. This means that for any $\alpha \leq 2$, the sequence $\left(D_{z}^{\alpha} f_{B_{k}}\right)$ converges to $D_{z}^{\alpha} \bar{f}$ with respect to the weak-*-topology on $\left[L^{1}\left(0, T ; L^{\beta^{\prime}}\right)\right]^{*} \widehat{=} L^{\infty}\left(0, T ; L^{\beta}\right)$ where $1 / \beta+1 / \beta^{\prime}=1$. Because of uniqueness of the limit it holds that $D_{z}^{\alpha} f=D_{z}^{\alpha} \bar{f}$ and thus

$$
f=\bar{f} \in W^{1,2}\left(0, T ; C_{b}\right) \cap C\left([0, T] ; C_{b}^{1}\right) \cap L^{\infty}\left(0, T ; W^{2, \beta}\right) .
$$

To show that $f$ is a strong solution to the field $B$, we have to verify the conditions from Definition 17. Uniqueness then follows directly from Proposition 18.

Condition (ii): For any measurable subset $M \subset[0, T] \times \mathbb{R}^{n}$,

$$
\begin{aligned}
\int_{M} \mid & \partial_{t} f+v \cdot \partial_{x} f-\partial_{x} \psi_{f} \cdot \partial_{v} f+(v \times B) \cdot \partial_{v} f \mid \mathrm{d}(t, x, v) \\
= & \int_{M} \mid\left(\partial_{t} f+v \cdot \partial_{x} f-\partial_{x} \psi_{f} \cdot \partial_{v} f+(v \times B) \cdot \partial_{v} f\right) \\
& -\left(\partial_{t} f_{B_{k}}+v \cdot \partial_{x} f_{B_{k}}-\partial_{x} \psi_{f_{B_{k}}} \cdot \partial_{v} f_{B_{k}}+\left(v \times B_{k}\right) \cdot \partial_{v} f_{B_{k}}\right) \mid \mathrm{d}(t, x, v) \\
\leq & C\left\|\partial_{t} f-\partial_{t} f_{B_{k}}\right\|_{\infty}+C\left\|\partial_{x} f-\partial_{x} f_{B_{k}}\right\|_{\infty} \\
& +C \int_{0}^{T}\left(\left\|\partial_{x} \psi_{f}(t)\right\|_{\infty}+R\|B(t)\|_{\infty}\right) \mathrm{d} t\left\|\partial_{v} f-\partial_{v} f_{B_{k}}\right\|_{\infty} \\
& +C\left\|\partial_{v} f_{B_{k}}\right\|_{\infty} \int_{0}^{T}\left(\left\|\partial_{x} \psi_{f_{B_{k}}-f}(t)\right\|_{\infty}+R\left\|B(t)-B_{k}(t)\right\|_{\infty}\right) \mathrm{d} t \\
\leq & C\left\|f-f_{B_{k}}\right\|_{W^{1,2}\left(0, T ; C_{b}\right)}+C\left\|f-f_{B_{k}}\right\|_{C\left([0, T] ; C_{b}^{1}\right)}+C\left\|_{B}-B_{k}\right\|_{L^{2}\left(0, T ; W^{2, \beta}\right)} \\
\leq & C\left\|B-B_{k}\right\|_{L^{2}\left(0, T ; W^{2, \beta}\right)}^{\gamma} \rightarrow 0,
\end{aligned}
$$

if $k \rightarrow \infty$. This means that $\partial_{t} f+v \cdot \partial_{x} f-\partial_{x} \psi_{f} \cdot \partial_{v} f+(v \times B) \cdot \partial_{v} f=0$ for almost every $(t, x, v) \in[0, T] \times \mathbb{R}^{6}$ that is condition (ii).

Item (b): Obviously,

$$
|f(0)-\stackrel{\circ}{f}|=\left|f(0)-f_{B_{k}}(0)\right| \leq C\left\|f-f_{B_{k}}\right\|_{C\left([0, T] ; C_{b}\right)} \rightarrow 0, \quad k \rightarrow \infty,
$$

so $f(0)=\stackrel{\circ}{f}$ everywhere on $\mathbb{R}^{6}$ that is $(\mathrm{c})$.

Condition (iii): Of course (b) directly implies condition (iii) from Definition 17.

Condition (iv): Due to uniform convergence and continuity of $f$, it is evident that $f(t)$ is also compactly supported in $B_{R}(0)$ for every $t \in[0, T]$.

Item (a): For $1 \leq q \leq \infty$ arbitrary, $t \in[0, T]$ and $k \in \mathbb{N}$, we have

$$
\begin{aligned}
& \left|\|f(t)\|_{q}-\|\stackrel{\circ}{f}\|_{q}\right|=\left|\|f(t)\|_{q}-\left\|f_{B_{k}}(t)\right\|_{q}\right| \leq\left\|f(t)-f_{B_{k}}(t)\right\|_{q} \\
& \quad \leq\left(\lambda\left(B_{R}(0)\right)\right)^{\frac{1}{q}}\left\|f(t)-f_{B_{k}}(t)\right\|_{\infty} \leq\left(1+\lambda\left(B_{R}(0)\right)\right)\left\|f-f_{B_{k}}\right\|_{C\left([0, T] ; C_{b}\right)} \rightarrow 0,
\end{aligned}
$$


if $k \rightarrow \infty$ where $\frac{1}{q} \widehat{=} 0$ if $q=\infty$. This means that $\|f(t)\|_{q}=\left\|f^{\circ}\right\|_{q}$ for every $t \in[0, T]$ and $1 \leq q \leq \infty$. Moreover we can choose some fixed $k \in \mathbb{N}$ such that

$$
\left\|f-f_{B_{k}}\right\|_{W^{1,2}\left(0, T ; C_{b}\right)}+\left\|f-f_{B_{k}}\right\|_{C\left([0, T] ; C_{b}^{1}\right)} \leq 1 .
$$

Also note that $\left\|f_{B_{k}}\right\|_{C\left([0, T] ; C_{b}^{1}\right)} \leq C,\left\|f_{B_{k}}\right\|_{W^{1,2}\left(0, T ; C_{b}\right)} \leq C$ and $\left\|D_{z}^{2} f_{B_{k}}\right\|_{L^{2}\left(0, T ; L^{2}\right)} \leq C$ by Lemma 15. It holds that

$$
\begin{aligned}
& \|f\|_{W^{1,2}\left(0, T ; C_{b}\right)}+\|f\|_{C\left([0, T] ; C_{b}^{1}\right)} \\
& \quad \leq\left\|f-f_{B_{k}}\right\|_{W^{1,2}\left(0, T ; C_{b}\right)}+\left\|f-f_{B_{k}}\right\|_{C\left([0, T] ; C_{b}^{1}\right)}+\left\|f_{B_{k}}\right\|_{W^{1,2}\left(0, T ; C_{b}\right)}+\left\|f_{B_{k}}\right\|_{C\left([0, T] ; C_{b}^{1}\right)} \\
& \quad \leq C .
\end{aligned}
$$

Moreover by the weak-* lower semicontinuity of the norm,

$$
\|f\|_{L^{\infty}\left(0, T ; W^{2, \beta}\right)} \leq \liminf _{k \rightarrow \infty}\left\|f_{B_{k}}\right\|_{L^{\infty}\left(0, T ; W^{2, \beta}\right)} \leq C
$$

This proves (a).

Condition (i): The condition (i) from Definition 17 follows directly from (a).

Thus the proof of Theorem 19 is complete.

Now that we have showed that any magnetic field $B \in \mathbb{B}_{K}$ yields a unique strong solution of the initial value problem (3.4), we can define an operator mapping every admissible field onto its induced state.

Definition 20 The operator

$$
f .: \mathbb{B}_{K} \rightarrow C\left([0, T] ; L^{2}\left(\mathbb{R}^{6}\right)\right), B \mapsto f_{B}
$$

is called the field-state operator. At this point $f_{B}$ denotes the unique strong solution of (3.4) that is induced by the field $B \in \mathbb{B}_{K}$.

From now on the notation $f_{B}$ is to be understood as the value of the field-state operator at point $B \in \mathbb{B}_{K}$. 


\section{Chapter 4}

\section{Continuity and compactness of the field-state operator}

Obviously the Lipschitz estimates of Lemma 16 hold true for the strong solutions by approximation.

Corollary 21 Let $L_{1}, L_{2}, L_{3}$ and $c_{5}$ be the constants from Lemma 15 and Lemma 16. Then for all $B, H \in \mathbb{B}_{K}$,

$$
\begin{aligned}
&\left\|f_{B}-f_{H}\right\|_{C\left([0, T] ; C_{b}\right)} \leq L_{1}\|B-H\|_{L^{2}\left(0, T ; W^{1, \beta}\left(B_{R_{Z}}(0)\right)\right)} \\
&\left\|\partial_{z} f_{B}-\partial_{z} f_{H}\right\|_{C\left([0, T] ; C_{b}\right)} \leq L_{2}\|B-H\|_{L^{2}\left(0, T ; W^{2, \beta}\left(B_{R_{Z}}(0)\right)\right)}^{\gamma} \\
&\left\|\partial_{t} f_{B}-\partial_{t} f_{H}\right\|_{L^{2}\left(0, T ; C_{b}\right)} \leq L_{3}\|B-H\|_{L^{2}\left(0, T ; W^{2, \beta}\left(B_{R_{Z}}(0)\right)\right)}^{\gamma} \\
&\left\|D_{z}^{2} f_{B}\right\|_{L^{\infty}\left(0, T ; W^{2, \beta}\right)} \leq c_{5} .
\end{aligned}
$$

This means that the field-state operator is globally Lipschitz-continuous with respect to the norm on $C\left([0, T] ; C_{b}\left(\mathbb{R}^{6}\right)\right)$ and globally Hölder-continuous with exponent $\gamma=\gamma(\beta)$ with respect to the norm on $W^{1,2}\left(0, T ; C_{b}\left(\mathbb{R}^{6}\right)\right)$ and the norm on $C\left([0, T] ; C_{b}^{1}\left(\mathbb{R}^{6}\right)\right)$.

The following proposition provides (weak) compactness of the field-state operator that will be very useful in terms of variational calculus.

Proposition 22 Let $\left(B_{k}\right)_{k \in \mathbb{N}} \subset \mathbb{B}_{K}$ be a sequence that is converging weakly in $L^{2}\left(0, T ; W^{2, \beta}\right)$ to some limit $B \in \mathbb{B}_{K}$. Then there exists a subsequence $\left(B_{k_{j}}\right)$ of $\left(B_{k}\right)$ such that

$f_{B_{k_{j}}} \rightarrow f_{B} \quad$ in $\quad W^{1,2}\left(0, T ; L^{p}\right) \cap L^{2}\left(0, T ; W^{1, p}\right) \cap L^{2}\left(0, T ; W^{2, \beta}\right) \quad$ for every $1 \leq p<\infty$, $f_{B_{k_{j}}} \rightarrow f_{B} \quad$ in $\quad L^{2}\left([0, T] \times \mathbb{R}^{6}\right)$

if $j$ tends to infinity.

Proof Let $\left(B_{k}\right)_{k \in \mathbb{N}} \subset \mathbb{B}_{K}$ and $B \in \mathbb{B}_{K}$ such that $B_{k} \rightarrow B$ in $L^{2}\left(0, T ; W^{2, \beta}\right)$. According to Theorem 19, $f_{k}:=f_{B_{k}}$ is bounded in $W^{1,2}\left(0, T ; L^{p}\right) \cap L^{2}\left(0, T ; W^{1, p} \cap W^{2, \beta}\right)$ for every $1 \leq p \leq \infty$. Note that this bound can be chosen independent of $p$. Hence the BanachAlaoglu theorem and Cantor's diagonal argument imply that, after extraction of a subsequence, $\left(f_{k}\right)$ is converging weakly in $W^{1,2}\left(0, T ; L^{m}\right) \cap L^{2}\left(0, T ; W^{1, m}\right) \cap L^{2}\left(0, T ; W^{2, \beta}\right)$ for every $m \in \mathbb{N}$ with $m \geq 2$. 
Thus there exists some function $f \in W^{1,2}\left(0, T ; L^{m}\right) \cap L^{2}\left(0, T ; W^{1, m}\right) \cap L^{2}\left(0, T ; W^{2, \beta}\right)$ for every $m \in \mathbb{N}, m \geq 2$ such that

$f_{k} \rightarrow f \quad$ in $\quad W^{1,2}\left(0, T ; L^{m}\right) \cap L^{2}\left(0, T ; W^{1, m}\right) \cap L^{2}\left(0, T ; W^{2, \beta}\right)$ for every $m \in \mathbb{N}, m \geq 2$

if $k \rightarrow \infty$. Thus, by interpolation, $f \in W^{1,2}\left(0, T ; L^{p}\right) \cap L^{2}\left(0, T ; W^{1, p}\right) \cap L^{2}\left(0, T ; W^{2, \beta}\right)$ for every $2 \leq p<\infty$. We will now show that $f$ is a strong solution to the field $B$ by verifying the conditions from Definition 17 .

Condition (iv): Let $\varepsilon>0$ be arbitrary. We will now assume that there exists some measurable set $M \subset[0, T] \times\left(\mathbb{R}^{6} \backslash B_{R}(0)\right)$ with Lebesgue-measure $\lambda(M)>0$ such that $f>\varepsilon$ almost everywhere on $M$. Then

$$
0<\varepsilon \lambda(M)<\int_{M} f \mathrm{~d}(t, z)=\int_{M} f-f_{k} \mathrm{~d}(t, z)=\int\left(f-f_{k}\right) \mathbb{1}_{M} \mathrm{~d}(t, z) \rightarrow 0, \quad k \rightarrow \infty
$$

which is a contradiction. The case $f<-\varepsilon$ can be treated analogously. Hence $-\varepsilon<$ $f<\varepsilon$ almost everywhere on $[0, T] \times\left(\mathbb{R}^{6} \backslash B_{R}(0)\right)$ which immediately yields $f=0$ almost everywhere on $[0, T] \times\left(\mathbb{R}^{6} \backslash B_{R}(0)\right)$ because $\varepsilon$ was arbitrary. Since $W^{1,2}\left(0, T ; L^{p}\right)$ is continuously embedded in $C\left([0, T] ; L^{p}\right)$ by Sobolev's embedding theorem, we have $\operatorname{supp} f(t) \subset B_{R}(0)$ even for all $t \in[0, T]$.

Condition (i): The fact that $\operatorname{supp} f(t) \subset B_{R}(0)$ for all $t \in[0, T]$ directly implies that $f \in W^{1,2}\left(0, T ; L^{p}\right) \cap L^{2}\left(0, T ; W^{1, p}\right)$ for every $1 \leq p<\infty$ by interpolation. Then we can easily conclude that

$f_{k} \rightarrow f \quad$ in $\quad W^{1,2}\left(0, T ; L^{p}\right) \cap L^{2}\left(0, T ; W^{1, p}\right) \cap L^{2}\left(0, T ; W^{2, \beta}\right) \quad$ for every $\quad 1 \leq p<\infty$.

The inequality

$$
\|f\|_{W^{1,2}\left(0, T ; L^{p}\left(\mathbb{R}^{6}\right)\right)}+\|f\|_{L^{2}\left(0, T ; W^{1, p}\right)} \leq C
$$

where $C>0$ depends only on $f, T, K$ and $\beta$ follows directly from the weak convergence and the weak lower semicontinuity of the norm. Since $C$ does not depend on $p$ this inequality holds true for $p=\infty$.

Condition (iii): It holds that $f_{k} \rightarrow f$ in $W^{1,2}\left(0, T ; L^{2}\right)$ with $f_{k}(0)=\stackrel{\circ}{f}$ almost everywhere on $\mathbb{R}^{6}$ for all $k \in \mathbb{N}$. By Mazur's lemma we can construct some sequence $\left(f_{k}^{*}\right)_{k \in \mathbb{N}}$ such that $f_{k}^{*} \rightarrow f$ in $W^{1,2}\left(0, T ; L^{2}\right)$ where for any $k \in \mathbb{N}, f_{k}^{*}$ is a convex combination of $f_{1}, \ldots, f_{k}$. Then of course $f_{k}^{*}(0)=\stackrel{\circ}{f}$ almost everywhere on $\mathbb{R}^{6}$ as well and hence

$$
\|f(0)-\stackrel{\circ}{f}\|_{L^{2}}=\left\|f(0)-f_{k}^{*}(0)\right\|_{L^{2}} \leq C\left\|f-f_{k}^{*}\right\|_{W^{1,2}\left(0, T ; L^{2}\right)} \rightarrow 0, \quad k \rightarrow \infty .
$$

Thus $f(0)=\stackrel{\circ}{f}$ almost everywhere on $\mathbb{R}^{6}$.

Condition (ii): We know that $f_{k} \rightarrow f$ in $W^{1,2}\left(0, T ; L^{2}\right) \cap L^{2}\left(0, T ; W^{1,2}\right)=H^{1}(] 0, T\left[\times \mathbb{R}^{6}\right)$ by Lemma 5 . Then, because of the compact support, the Rellich-Kondrachov theorem implies that $f_{k} \rightarrow f$ in $L^{2}\left([0, T] \times \mathbb{R}^{6}\right)$, up to a subsequence. From Proposition 8 we can conclude that for any $t \in[0, T]$,

$$
\left\|\partial_{x} \psi_{f}(t)-\partial_{x} \psi_{f_{k}}(t)\right\|_{L^{2}\left(B_{R}(0)\right)} \leq C\left\|f(t)-f_{k}(t)\right\|_{L^{2}} \rightarrow 0, \quad k \rightarrow \infty
$$


For brevity, we will now use the notation

$$
\mathcal{V}(\varphi, f, B):=\partial_{t} \varphi+v \cdot \partial_{x} \varphi-\partial_{x} \psi_{f} \cdot \partial_{v} \varphi+(v \times B) \cdot \partial_{v} \varphi
$$

Let $\left.\varphi \in C_{c}^{\infty}(] 0, T\left[\times \mathbb{R}^{6}\right)\right)$ be an arbitrary test function. Then $\mathcal{V}\left(\varphi, f_{k}, B_{k}\right)$ is bounded in $L^{2}(] 0, T\left[\times B_{R}(0)\right)$ uniformely in $k$ (the bound may depend on $\varphi$ ). It also holds that

$$
\mathcal{V}\left(\varphi, f, B_{k}\right)-V\left(\varphi, f_{k}, B_{k}\right) \rightarrow 0, \quad k \rightarrow \infty \quad \text { in } L^{2}(] 0, T\left[\times B_{R}(0)\right)
$$

since $\psi_{f_{k}} \rightarrow \psi_{f}$ in $L^{2}(] 0, T\left[\times B_{R}(0)\right)$. Moreover,

$$
\mathcal{V}(\varphi, f, B)-\mathcal{V}\left(\varphi, f, B_{k}\right) \rightarrow 0, \quad k \rightarrow \infty \quad \text { in } L^{2}(] 0, T\left[\times B_{R}(0)\right)
$$

Hence by integration by parts,

$$
\begin{aligned}
\mid \int_{0}^{T} \int & \mathcal{V}(f, f, B) \varphi \mathrm{d} z \mathrm{~d} t|=| \int_{0}^{T} \int f \mathcal{V}(\varphi, f, B) \mathrm{d} z \mathrm{~d} t \mid \\
= & \left|\int_{0}^{T} \int f \mathcal{V}(\varphi, f, B)-f_{k} \mathcal{V}\left(\varphi, f_{k}, B_{k}\right) \mathrm{d} z \mathrm{~d} t\right| \\
\leq & \left|\int_{0}^{T} \int f\left(\mathcal{V}(\varphi, f, B)-\mathcal{V}\left(\varphi, f, B_{k}\right)\right) \mathrm{d} z \mathrm{~d} t\right| \\
& +\left|\int_{0}^{T} \int f\left(\mathcal{V}\left(\varphi, f, B_{k}\right)-\mathcal{V}\left(\varphi, f_{k}, B_{k}\right)\right) \mathrm{d} z \mathrm{~d} t\right| \\
& +\left|\int_{0}^{T} \int\left(f-f_{k}\right) \mathcal{V}\left(\varphi, f_{k}, B_{k}\right) \mathrm{d} z \mathrm{~d} t\right| \\
\rightarrow & 0, k \rightarrow \infty .
\end{aligned}
$$

As $\varphi$ was arbitrary this implies that $\mathcal{V}(f, f, B)=0$ almost everywhere on $[0, T] \times \mathbb{R}^{6}$ that is (ii).

Consequently $f$ is a strong solution to the field $B$ and thus $f=f_{B}$ because of uniqueness. Furthermore we have showed that there exists a subsequence $\left(B_{k_{j}}\right)$ of $\left(B_{k}\right)$ such that $\left(f_{B_{k_{j}}}\right)$ is converging in the demanded fashion. 


\section{Chapter 5}

\section{Fréchet differentiability of the field-state operator}

Since the Fréchet derivative is a linear approximation of the field-state operator at some certain point $B \in \mathbb{B}_{K}$ it turns out that this derivative is determined by an inhomogenous linear Vlasov equation. In the following section we will analyze those linear Vlasov equations in general, i.e., we will establish some existence and uniqueness results. The type and the regularity of the solution will depend on the regularity of the coefficients.

\subsection{A general inhomogenous linear Vlasov equation}

Let $r_{0} \geq 0$ and $r_{2}>r_{1} \geq 0$ be arbitrary. We consider the following inhomogenous linear version of the Vlasov equation:

$$
\left\{\begin{array}{l}
\partial_{t} f+v \cdot \partial_{x} f+\mathbf{A} \cdot \partial_{v} f+(v \times \mathbf{B}) \cdot \partial_{v} f=\partial_{x} \psi_{f} \cdot \mathbf{C}+\chi \Phi_{\mathbf{a}, f}+\mathbf{b} \\
\left.f\right|_{t=0}=\AA
\end{array}\right.
$$

The coefficients are supposed to have the following regularity:

$$
\begin{cases}\mathbf{a}=\mathbf{a}(t, x, v) \in C\left([0, T] ; C_{b}^{1}\left(\mathbb{R}^{6}\right)\right), & \operatorname{supp} \mathbf{a}(t) \subset B_{r_{0}}(0), t \in[0, T], \\ \mathbf{b}=\mathbf{b}(t, x, v) \in C\left([0, T] ; C_{b}^{1}\left(\mathbb{R}^{6}\right)\right), & \text { supp } \mathbf{b}(t) \subset B_{r_{0}}(0), t \in[0, T], \\ \stackrel{\circ}{\mathbf{f}}=\stackrel{\circ}{\mathbf{f}}(x, v) \in C_{c}^{2}\left(\mathbb{R}^{6}\right), & \text { supp } \mathbf{f} \subset B_{r_{0}}(0), \\ \mathbf{A}=\mathbf{A}(t, x) \in C\left([0, T] ; C^{1, \gamma}\left(\mathbb{R}^{3} ; \mathbb{R}^{3}\right)\right), & \\ \mathbf{B}=\mathbf{B}(t, x) \in C\left([0, T] ; C^{1, \gamma}\left(\mathbb{R}^{3} ; \mathbb{R}^{3}\right)\right), & \\ \mathbf{C}=\mathbf{C}(t, x, v) \in C\left(0, T ; C_{b}^{1}\left(\mathbb{R}^{6} ; \mathbb{R}^{3}\right)\right), & \operatorname{supp} \mathbf{C}(t) \subset B_{r_{0}}(0), t \in[0, T], \\ \chi=\chi(x, v) \in C_{c}^{1}\left(\mathbb{R}^{6} ;[0,1]\right), & \chi=1 \text { on } B_{r_{1}}(0), \operatorname{supp} \chi \subset B_{r_{2}}(0)\end{cases}
$$

Moreover $\Phi_{\mathbf{a}, f}$ is given by

$$
\Phi_{\mathbf{a}, f}(t, x):=-\iint \frac{x-y}{|x-y|^{3}} \cdot \partial_{v} \mathbf{a}(t, y, w) f(t, y, w) \mathrm{d} w \mathrm{~d} y, \quad(t, x) \in[0, T] \times \mathbb{R}^{3} .
$$


For $(t, x) \in[0, T] \times \mathbb{R}^{3}$ we will also use the notation

$$
\Phi_{\mathbf{a}, f}^{\prime}(t, x):=-\iint \frac{x-y}{|x-y|^{3}} \cdot\left(\partial_{v} \mathbf{a} \partial_{x} f-\partial_{v} f \partial_{x} \mathbf{a}\right)(t, y, w) \mathrm{d} w \mathrm{~d} y .
$$

Note that

$$
\Phi_{\mathbf{a}, f}=\sum_{i=1}^{3} \partial_{x_{i}} \psi_{\partial_{v_{i}}} \mathbf{a} f \quad \text { and } \quad\left[\Phi_{\mathbf{a}, f}^{\prime}\right]_{j}=\sum_{i=1}^{3} \partial_{x_{i}} \psi_{\partial_{v_{i}} \mathbf{a} \partial_{x_{j}} f-\partial_{v_{i}} f \partial_{x_{j}} \mathbf{a}}, \quad j=1,2,3 .
$$

As $\mathbf{a} \in C\left([0, T] ; C_{b}^{1}\left(\mathbb{R}^{6}\right)\right)$ with compact support supp a $(t) \subset B_{r_{0}}(0)$ for all $t \in[0, T]$, Proposition 8 provides the following inequalities: For any $r>0$ there exists some constant $c>0$ that may depend only on $r$ and $r_{0}$ such that for almost all $t \in[0, T]$,

$$
\begin{array}{ll}
\left\|\Phi_{\mathbf{a}, f}(t)\right\|_{L^{2}\left(B_{r}(0)\right)} \leq c\left\|\partial_{v} a(t)\right\|_{\infty}\|f(t)\|_{L^{2}\left(B_{r_{0}}(0)\right)}, & f \in L^{2}\left(0, T ; L^{2}\right), \\
\left\|\Phi_{\mathbf{a}, f}^{\prime}(t)\right\|_{L^{2}\left(B_{r}(0)\right)} \leq c\left\|\partial_{z} a(t)\right\|_{\infty}\left\|\partial_{z} f(t)\right\|_{L^{2}\left(B_{r_{0}}(0)\right),}, & f \in L^{2}\left(0, T ; H^{1}\right), \\
\left\|\Phi_{\mathbf{a}, f}(t)\right\|_{L^{\infty}} \leq c\left\|\partial_{v} a(t)\right\|_{\infty}\|f(t)\|_{L^{\infty}\left(B_{r_{0}}(0)\right)}, & f \in L^{2}\left(0, T ; L^{\infty}\right), \\
\left\|\Phi_{\mathbf{a}, f}^{\prime}(t)\right\|_{L^{\infty}} \leq c\left\|\partial_{z} a(t)\right\|_{\infty}\left\|\partial_{z} f(t)\right\|_{L^{\infty}\left(B_{r_{0}}(0)\right),}, & f \in L^{2}\left(0, T ; W^{1, \infty}\right) .
\end{array}
$$

If $\mathbf{a} \in C\left([0, T] ; C_{b}^{2}\left(\mathbb{R}^{6}\right)\right)$ and $f \in C\left([0, T] ; C_{b}^{1}\left(\mathbb{R}^{6}\right)\right)$ then $\Phi_{\mathbf{a}, f}$ is continuously differentiable with respect to $x$ with

$$
\begin{aligned}
\partial_{x_{j}} \Phi_{\mathbf{a}, f}(t, x) & =\sum_{i=1}^{3} \partial_{x_{j}} \partial_{x_{i}} \psi_{\partial_{v_{i}} \mathbf{a} f}=\sum_{i=1}^{3} \partial_{x_{i}} \psi_{\partial_{v_{i}} \mathbf{a} \partial_{x_{j}} f+\partial_{v_{i}} \partial_{x_{j}} \mathbf{a} f} \\
& =\sum_{i=1}^{3} \partial_{x_{i}} \psi_{\partial_{v_{i}}} \mathbf{a} \partial_{x_{j}} f-\partial_{x_{j}} \mathbf{a} \partial_{v_{i}} f=\left[\Phi_{\mathbf{a}, f}^{\prime}\right]_{j}(t, x)
\end{aligned}
$$

for all $(t, x) \in[0, T] \times \mathbb{R}^{3}$. Because of density this result holds true if $\mathbf{a} \in C\left([0, T] ; C_{b}^{1}\left(\mathbb{R}^{6}\right)\right)$. If merely $f \in L^{2}\left(0, T ; H^{1}\right)$ the result holds true in the weak sense.

Lemma 23 Let $A, B \in C\left([0, T] ; C_{b}^{1}\left(\mathbb{R}^{3} ; \mathbb{R}^{3}\right)\right)$ be arbitrary. Then for any $t \in[0, T]$ and $z \in \mathbb{R}^{6}$ the characteristic system

$$
\left\{\begin{array}{l}
\dot{x}=v \\
\dot{v}=\mathbf{A}(s, x)+v \times \mathbf{B}(t, x)
\end{array}\right.
$$

has a unique solution $Z \in C^{1}\left([0, T] \times[0, T] \times \mathbb{R}^{6} ; \mathbb{R}^{6}\right)$ with $Z(s, t, z)=(X, V)(s, t, z)$ to the initial value condition $Z(t, t, z)=z$.

For any $r>0$ and all $s, t \in[0, T], Z\left(s, t, B_{r}(0)\right) \subset B_{\zeta(r)}(0)$ where

$$
\zeta(r):=\mathrm{e}^{2 T}\left(r+\sqrt{T}\|\mathbf{A}\|_{L^{2}\left(0, T ; L^{\infty}\right)}\right)
$$

Moreover there exists some positive constant $C(r)$ depending only on $r,\|\mathbf{A}\|_{L^{2}\left(0, T ; C_{b}^{1}\right)}$, $\|\mathbf{B}\|_{L^{2}\left(0, T ; C_{b}^{1}\right)}$ such that for all $s, t \in[0, T]$,

$$
\left\|\partial_{z} Z(s, t, \cdot)\right\|_{L^{\infty}\left(B_{r}(0)\right)} \leq C(r) \quad \text { and } \quad\left\|\partial_{t} Z(s, t, \cdot)\right\|_{L^{\infty}\left(B_{r}(0)\right)} \leq C(r) .
$$


Proof The existence of a unique solution $Z \in C^{1}\left([0, T] \times[0, T] \times \mathbb{R}^{6} ; \mathbb{R}^{6}\right)$ to the initial value condition $Z(t, t, z)=z$ is obvious. Let now $s, t \in[0, T]$ be arbitrary. Without loss of generality, $s \leq t$. If $z \in B_{r}(0)$,

$$
\begin{aligned}
|Z(s, t, z)|^{2} & \leq r^{2}+2 \int_{s}^{t}|X(\tau, t, z)||V(\tau, t, z)|+|V(\tau, t, z)||\mathbf{A}(\tau, X(\tau, t, z))| \mathrm{d} \tau \\
& \leq r^{2}+2 \int_{s}^{t}|Z(\tau, t, z)|\|\mathbf{A}(\tau)\|_{\infty} \mathrm{d} \tau+2 \int_{s}^{t}|Z(\tau, t, z)|^{2} \mathrm{~d} \tau
\end{aligned}
$$

Thus by Gronwall's lemma

$$
|Z(s, t, z)|^{2} \leq \mathrm{e}^{2 T} r^{2}+2 \mathrm{e}^{2 T} \int_{s}^{t}|Z(\tau, t, z)|\|\mathbf{A}(\tau)\|_{\infty} \mathrm{d} \tau
$$

and then the quadratic version of Gronwall's lemma yields

$$
\|Z(s, t, \cdot)\|_{L^{\infty}\left(B_{r}(0)\right)} \leq \sqrt{\mathrm{e}^{2 T} r^{2}}+\mathrm{e}^{2 T} \sqrt{T}\|\mathbf{A}\|_{L^{2}\left(0, T ; L^{\infty}\right)}<\zeta(r) .
$$

Now let $C(r)$ denote a generic positive constant depending only on $r,\|\mathbf{A}\|_{L^{2}\left(0, T ; C_{b}^{1}\right)}$, $\|\mathbf{B}\|_{L^{2}\left(0, T ; C_{b}^{1}\right)}$. For any $i \in\{1, \ldots, 6\}$ and $z \in B_{r}(0)$,

$$
\begin{aligned}
&\left|\partial_{z_{i}} Z(s, t, z)\right| \leq 1+\int_{s}^{t}\left|\partial_{z_{i}} V(\tau)\right|+\left|D_{z} \mathbf{A}(\tau, X(\tau)) \cdot \partial_{z_{i}} X(\tau)\right| \\
&+|V(\tau)|\left|D_{z} \mathbf{B}(\tau, X(\tau)) \cdot \partial_{z_{i}} X(\tau)\right|+\left|\partial_{z_{i}} V(\tau)\right||\mathbf{B}(\tau, X(\tau))| \mathrm{d} \tau \\
& \leq C(r)+C(r) \int_{s}^{t}\left(1+\|\mathbf{A}(\tau)\|_{C_{b}^{1}}+\|\mathbf{B}(\tau)\|_{C_{b}^{1}}\right)\left|\partial_{z_{i}} Z(\tau, t, z)\right| \mathrm{d} \tau
\end{aligned}
$$

for all $i \in\{1, \ldots, 6\}$ and hence

$$
\left\|\partial_{z} Z(s, t,)\right\|_{L^{\infty}\left(B_{r}(0)\right)} \leq C(r) \int_{s}^{t}\|\mathbf{A}(\tau)\|_{C_{b}^{1}}+\|\mathbf{B}(\tau)\|_{C_{b}^{1}} \mathrm{~d} \tau \leq C(r) .
$$

One can easily show that $\partial_{t} Z(s, t, z)$ is given by

$$
\partial_{t} Z(s, t, z)=-D_{z} Z(s, t, z)\left(\begin{array}{c}
v \\
\mathbf{A}(t, z)+v \times \mathbf{B}(t, z)
\end{array}\right) .
$$

For more detail confer [9, p. 14]. Thus we also have $\left\|\partial_{t} Z(s, t,)\right\|_{L^{\infty}\left(B_{r}(0)\right)} \leq C(r)$ for all $i \in\{1, \ldots, 6\}$.

Now we can establish an existence and uniqueness result for classical solutions of the system (5.1) if the regularity conditions (5.2) hold. Unfortunately the coefficients of the systems that will occur in this paper do not satisfy those strong conditions. However, we will still be able to prove an existence and uniqueness result for strong solutions of (5.1) if the regularity conditions are slightly weaker. 
Proposition 24 Suppose that the coefficients of the system (5.1) satisfy the regularity condition (5.2). Then the initial value problem (5.1) has a unique classical solution $f \in C^{1}\left([0, T] \times \mathbb{R}^{6}\right)$. Moreover for all $t \in[0, T]$, supp $f(t) \subset B_{\zeta(r+1)}(0)$ with $r=\max \left\{r_{0}, r_{2}\right\}$ and $f$ is implicitely given by

$$
f(t, z)=\stackrel{\circ}{\mathbf{f}}(Z(0, t, z))+\int_{0}^{t}\left[\partial_{x} \psi_{f} \cdot \mathbf{C}+\chi \Phi_{\mathbf{a}, f}+\mathbf{b}\right](s, Z(s, t, z)) \mathrm{d} s
$$

for any $t \in[0, T], z \in \mathbb{R}^{6}$. Moreover there exists some constant $C>0$ depending only on $T, r_{0}, r_{2},\|\mathbf{a}\|_{C\left([0, T] ; C_{b}^{1}\right)},\|\mathbf{b}\|_{C\left([0, T] ; C_{b}^{1}\right)},\|\stackrel{f}{\mathbf{f}}\|_{C_{b}^{2}},\|\mathbf{A}\|_{C\left([0, T] ; C^{1, \gamma}\right)},\|\mathbf{B}\|_{C\left([0, T] ; C^{1, \gamma}\right)}$, $\|\mathbf{C}\|_{C\left([0, T] ; C_{b}^{1}\right)}$ and $\|\chi\|_{C_{b}^{1}}$ such that $\|f\|_{C_{b}^{1}\left([0, T] \times \mathbb{R}^{6}\right)} \leq C$.

The proof of Proposition 24 is very technical and is outsourced to the appendix.

\section{Comment}

(a) If we use a final value condition $\left.f\right|_{t=T}=\stackrel{\circ}{\mathbf{f}}$ instead of the initial value condition $\left.f\right|_{t=0}=\stackrel{\mathrm{f}}{ }$ the problem can be treated completely analogously. The results of Proposition 24 and Corollary 26 hold true in this case. Only the implicit depiction of a classical solution must be replaced by

$$
f(t, z)=\stackrel{\circ}{\mathbf{f}}(Z(T, t, z))-\int_{t}^{T}\left[\partial_{x} \psi_{f} \cdot \mathbf{C}+\chi \Phi_{\mathbf{a}, f}+\mathbf{b}\right](s, Z(s, t, z)) \mathrm{d} s
$$

(b) Suppose that $\mathbf{C}=0$ and recall that $\Phi_{\mathbf{a}, f}$ depends only on $\left.f\right|_{B_{r_{0}}(0)}$. Hence, if we choose $r_{1}=\zeta\left(r_{0}\right)$ then for all $t \in[0, T]$ and $z \in B_{r_{0}}(0)$,

$$
f(t, z)=\stackrel{\circ}{\mathbf{f}}(Z(0, t, z))+\int_{0}^{t}\left[\Phi_{\mathbf{a}, f}+\mathbf{b}\right](s, Z(s, t, z)) \mathrm{d} s
$$

because in this case $\chi(Z(s, t, z))=1$ as $Z\left(s, t, B_{r_{0}}(0)\right) \subset B_{r_{1}}(0)$. This means that the values of $\left.f\right|_{B_{r_{0}}(0)}$ do not depend on the choice of $\chi$ as long as $\chi=1$ on $B_{r_{1}}(0)$.

Definition 25 We call $f$ a strong solution of the initial value problem (5.1) iff the following holds:

(i) $f \in H^{1}(] 0, T\left[\times \mathbb{R}^{6}\right) \subset C\left([0, T] ; L^{2}\right)$.

(ii) $f$ satisfies

$$
\partial_{t} f+v \cdot \partial_{x} f+\mathbf{A} \cdot \partial_{v} f+(v \times \mathbf{B}) \cdot \partial_{v} f=\partial_{x} \psi_{f} \cdot \mathbf{C}+\Phi_{\mathbf{a}, f}+\mathbf{b}
$$

almost everywhere on $[0, T] \times \mathbb{R}^{6}$.

(iii) $f$ satisfies the initial condition $\left.f\right|_{t=0}=\stackrel{\circ}{\mathbf{f}}$ almost everywhere on $\mathbb{R}^{6}$.

(iv) There exists some radius $r>0$ such that for all $t \in[0, T]$, supp $f(t) \subset B_{r}(0)$. 


\section{Corollary 26}

(a) Suppose that the coefficients satisfy the following conditions:

$$
\begin{cases}\mathbf{a}=\mathbf{a}(t, x, v) \in C\left([0, T] ; C_{b}^{1}\left(\mathbb{R}^{6}\right)\right), & \operatorname{supp} \mathbf{a}(t) \subset B_{r_{0}}(0), t \in[0, T], \\ \mathbf{b}=\mathbf{b}(t, x, v) \in L^{2}\left(0, T ; C_{b} \cap H^{1}\left(\mathbb{R}^{6}\right)\right), & \operatorname{supp} \mathbf{b}(t) \subset B_{r_{0}}(0), t \in[0, T], \\ \stackrel{\circ}{\mathbf{f}} \mathbf{\mathbf { f }}(x, v) \in C_{c}^{1}\left(\mathbb{R}^{6}\right), & \operatorname{supp} \mathbf{f} \subset B_{r_{0}}(0), \\ \mathbf{A}=\mathbf{A}(t, x) \in C\left([0, T] ; C^{1, \gamma}\left(\mathbb{R}^{3} ; \mathbb{R}^{3}\right)\right), & \\ \mathbf{B}=\mathbf{B}(t, x) \in L^{2}\left(0, T ; C^{1, \gamma}\left(\mathbb{R}^{3} ; \mathbb{R}^{3}\right)\right), & \\ \mathbf{C}=\mathbf{C}(t, x, v) \in L^{2}\left(0, T ; H^{1} \cap C_{b}\left(\mathbb{R}^{6} ; \mathbb{R}^{3}\right)\right), \operatorname{supp} \mathbf{C}(t) \subset B_{r_{0}}(0), t \in[0, T], \\ \chi=\chi(x, v) \in C_{c}^{1}\left(\mathbb{R}^{6} ;[0,1]\right), & \chi=1 \text { on } B_{r_{1}}(0), \operatorname{supp} \chi \subset B_{r_{2}}(0)\end{cases}
$$

Then the following holds:

(A) There exist sequences

$$
\begin{array}{lll}
\left(\mathbf{b}_{k}\right) \subset C\left([0, T] ; C_{b}^{1}\right) & \text { with } & \mathbf{b}_{k} \rightarrow \mathbf{b} \text { in } L^{2}\left(0, T ; C_{b} \cap H^{1}\right), \\
\left(\stackrel{\mathbf{f}}{k}_{k}\right) \subset C_{c}^{2}\left(\mathbb{R}^{6}\right) & \text { with } & \mathbf{f}_{k} \rightarrow \mathbf{f} \text { in } C_{b}^{1}\left(\mathbb{R}^{6}\right), \\
\left(\mathbf{B}_{k}\right) \subset C\left([0, T] ; C^{1, \gamma}\right) & \text { with } & \mathbf{B}_{k} \rightarrow \mathbf{B} \text { in } L^{2}\left(0, T ; C^{1, \gamma}\right), \\
\left(\mathbf{C}_{k}\right) \subset C\left([0, T] ; C_{b}^{1}\right) & \text { with } & \mathbf{C}_{k} \rightarrow \mathbf{C} \text { in } L^{2}\left(0, T ; C_{b} \cap H^{1}\right)
\end{array}
$$

such that

$$
\operatorname{supp} \mathbf{b}_{k}(t), \operatorname{supp} \stackrel{\circ}{\mathbf{f}}_{k}, \operatorname{supp} \mathbf{C}_{k}(t) \subset B_{r_{0}+1}(0)
$$

for almost all $t \in[0, T]$ and all $k \in \mathbb{N}$.

For any $k \in \mathbb{N}$, there exists a unique classical solution $f_{k}$ of (5.1) to the coefficients $\mathbf{a}, \mathbf{b}_{k}, \stackrel{\circ}{f}_{k}, \mathbf{A}, \mathbf{B}_{k}, \mathbf{C}_{k}$ and $\boldsymbol{\chi}$. Moreover, there exists some constant $C>0$ depending only on $T, r_{0}, r_{2},\|\mathbf{a}\|_{C\left([0, T] ; C_{b}^{1}\right)},\|\mathbf{b}\|_{L^{2}\left(0, T ; C_{b}\right)},\|\mathbf{b}\|_{L^{2}\left(0, T ; H^{1}\right)}$, $\|\stackrel{f}{\mathbf{f}}\|_{C_{b}^{1}},\|\mathbf{A}\|_{C\left([0, T] ; C^{1, \gamma}\right)},\|\mathbf{B}\|_{L^{2}\left(0, T ; C^{1, \gamma}\right)},\|\chi\|_{C_{b}^{1}}$ and $\|\mathbf{C}\|_{L^{2}\left(0, T ; C_{b} \cap H^{1}\right)}$ such that

$$
\left\|f_{k}\right\|_{L^{\infty}(] 0, T\left[\times \mathbb{R}^{6}\right)}+\left\|f_{k}\right\|_{H^{1}(] 0, T\left[\times \mathbb{R}^{6}\right)} \leq C .
$$

(B) There exists some function $f \in L^{\infty} \cap H^{1}(] 0, T\left[\times \mathbb{R}^{6}\right)$ such that

$$
\|f\|_{L^{\infty}(] 0, T\left[\times \mathbb{R}^{6}\right)}+\|f\|_{H^{1}(] 0, T\left[\times \mathbb{R}^{6}\right)} \leq C
$$

and

$$
f_{k} \stackrel{*}{\rightarrow} f \in L^{\infty}(] 0, T\left[\times \mathbb{R}^{6}\right), \quad f_{k} \rightarrow f \in H^{1}(] 0, T\left[\times \mathbb{R}^{6}\right)
$$

if $k \rightarrow \infty$ up to a subsequence.

(C) The function $f$ is a unique strong solution of the initial value problem (5.1). For almost all $t \in[0, T]$, supp $f(t) \subset B_{\zeta(3+r)}(0)$ with $r=\max \left\{r_{0}, r_{2}\right\}$. 
(b) Suppose that the coefficients satisfy the following conditions:

$$
\begin{cases}\mathbf{a}=\mathbf{a}(t, x, v) \in C\left([0, T] ; C_{b}^{1}\left(\mathbb{R}^{6}\right)\right), & \text { supp } \mathbf{a}(t) \subset B_{r_{0}}(0), t \in[0, T], \\ \mathbf{b}=0, & \\ \stackrel{\circ}{\mathbf{f}}=\mathbf{f}(x, v) \in C_{c}^{2}\left(\mathbb{R}^{6}\right), & \\ \mathbf{A}=\mathbf{A}(t, x) \in C\left([0, T] ; C^{1, \gamma}\left(\mathbb{R}^{3} ; \mathbb{R}^{3}\right)\right), & \\ \mathbf{B}=\mathbf{B}(t, x) \in L^{2}\left(0, T ; C^{1, \gamma}\left(\mathbb{R}^{3} ; \mathbb{R}^{3}\right)\right), & \\ \mathbf{C}=0, & \chi=1 \text { on } B_{r_{1}}(0), \operatorname{supp} \chi \subset B_{r_{2}}(0) \\ \chi=\chi(x, v) \in C_{c}^{1}\left(\mathbb{R}^{6} ;[0,1]\right), & \end{cases}
$$

Then the following holds:

(A) There exists a sequence

$$
\left(\mathbf{B}_{k}\right) \subset C\left([0, T] ; C^{1, \gamma}\right) \quad \text { with } \quad \mathbf{B}_{k} \rightarrow \mathbf{B} \text { in } L^{2}\left(0, T ; C^{1, \gamma}\right) .
$$

For any $k \in \mathbb{N}$, there exists a unique classical solution $f_{k}$ of (5.1) to the coeffcients $\mathbf{a}, \mathbf{b}=0, \stackrel{\mathbf{f}}{\mathbf{A}} \mathbf{A}, \mathbf{B}_{k}, \mathbf{C}=0$ and $\boldsymbol{\chi}$. Moreover, there exists some constant $C>0$ depending only on $T, r_{0}, r_{2},\|\mathbf{a}\|_{C\left([0, T] ; C_{b}^{1}\right)},\|\mathbf{f}\|_{C_{b}^{2}},\|\mathbf{A}\|_{C\left([0, T] ; C^{1, \gamma}\right)}$, $\|\mathbf{B}\|_{L^{2}\left(0, T ; C^{1, \gamma}\right)}$ and $\|\chi\|_{C_{b}^{1}}$ such that for all $k, j \in \mathbb{N}$,

$$
\begin{gathered}
\left\|f_{k}-f_{j}\right\|_{C\left([0, T] ; C_{b}\right)} \leq C\left\|\mathbf{B}_{k}-\mathbf{B}_{j}\right\|_{L^{2}\left(0, T ; C^{1, \gamma}\right)}, \\
\left\|\partial_{z} f_{k}-\partial_{z} f_{j}\right\|_{C\left([0, T] ; C_{b}\right)} \leq C\left\|\mathbf{B}_{k}-\mathbf{B}_{j}\right\|_{L^{2}\left(0, T ; C^{1, \gamma}\right)}^{\gamma}, \\
\left\|\partial_{t} f_{k}-\partial_{t} f_{j}\right\|_{L^{2}\left(0, T ; C_{b}\right)} \leq C\left\|\mathbf{B}_{k}-\mathbf{B}_{j}\right\|_{L^{2}\left(0, T ; C^{1, \gamma}\right)}^{\gamma}
\end{gathered}
$$

(B) There exists some function $f \in W^{1,2}\left(0, T ; C_{b}\right) \cap C\left([0, T] ; C_{b}^{1}\right)$ such that

$$
f_{k} \rightarrow f \in W^{1,2}\left(0, T ; C_{b}\right), \quad f_{k} \rightarrow f \in C\left([0, T] ; C_{b}^{1}\right)
$$

if $k \rightarrow \infty$ up to a subsequence. Moreover, there exists some constant $C>0$ depending only on $T, r_{0}, r_{2},\|\mathbf{a}\|_{C\left([0, T] ; C_{b}^{1}\right)},\|\stackrel{\mathbf{f}}{ }\|_{C_{b}^{2}},\|\mathbf{A}\|_{C\left([0, T] ; C^{1, \gamma}\right)},\|\chi\|_{C_{b}^{1}}$ and $\|\mathbf{B}\|_{L^{2}\left(0, T ; C^{1, \gamma}\right)}$ such that

$$
\|f\|_{W 1,2\left(0, T ; C_{b}\right)}+\|f\|_{C\left([0, T] ; C_{b}^{1}\right)} \leq C .
$$

(C) The function $f$ is a unique strong solution of the initial value problem (5.1). For almost all $t \in[0, T], \operatorname{supp} f(t) \subset B_{\zeta(2+r)}(0)$ with $r=\max \left\{r_{0}, r_{2}\right\}$.

(D) If we fix $r_{1}:=\zeta\left(r_{0}\right)$ then $\left.f\right|_{B_{r_{0}}(0)}$ does not depend on the choice of $\chi$ as long as $\chi=1$ on $B_{r_{1}}(0)$.

The proof of this Corollary can also be found in the appendix. 


\subsection{The Fréchet derivative of the field-state operator}

Again, let $K>0$ be arbitrary. We can now use the results of Section 5.1 to establish Fréchet differentiability of the control state operator on $\mathbb{B}_{K}$ (that is the interior of $\mathbb{B}_{K}$ ).

Theorem 27 Let $f$. be the field-state operator as defined in Definition 20. For $B \in \mathbb{B}_{K}$ and $H \in \mathcal{V}$ there exists a unique strong solution $f_{B}^{H} \in L^{\infty} \cap H^{1}(] 0, T[\times \mathbb{R}) \subset C\left([0, T] ; L^{2}\right)$ of the initial value problem

$$
\left\{\begin{array}{l}
\partial_{t} f+v \cdot \partial_{x} f-\partial_{x} \psi_{f_{B}} \cdot \partial_{v} f-\partial_{x} \psi_{f} \cdot \partial_{v} f_{B}+(v \times B) \cdot \partial_{v} f+(v \times H) \cdot \partial_{v} f_{B}=0 \\
\left.f\right|_{t=0}=0
\end{array}\right.
$$

with supp $f(t) \subset B_{\varrho}(0)$ for all $t \in[0, T]$ and some radius $\varrho>0$ depending only on $T, K, \stackrel{\circ}{f}$ and $\beta$. Then the following holds:

(a) Let $t \in[0, T]$ be arbitrary. Then $f .(t)$ is Fréchet differentiable on $\stackrel{\circ}{\mathbb{B}}_{K}$ with respect to the $L^{2}\left(\mathbb{R}^{6}\right)$-norm, i.e., for any $B \in \stackrel{\circ}{B}_{K}$ there exists a unique linear operator $f_{B}^{\prime}(t): \mathcal{V} \rightarrow L^{2}\left(\mathbb{R}^{6}\right)$ such that

$$
\begin{gathered}
\forall \varepsilon>0 \exists \delta>0 \forall H \in \mathcal{V} \text { with }\|H\|_{\mathcal{V}}<\delta: \\
B+H \in \stackrel{\circ}{\mathbb{B}}_{K} \quad \text { and } \quad \frac{\left\|f_{B+H}(t)-f_{B}(t)-f_{B}^{\prime}(t)[H]\right\|_{L^{2}}}{\|H\|_{\mathcal{V}}}<\varepsilon .
\end{gathered}
$$

The Fréchet derivative is given by

$$
f_{B}^{\prime}(t)[H]=f_{B}^{H}(t), \quad H \in \mathcal{V} .
$$

(b) The field-state operator $f$. is Fréchet differentiable on $\stackrel{\circ}{\mathbb{B}}_{K}$ with respect to the $C\left([0, T] ; L^{2}\left(\mathbb{R}^{6}\right)\right)$-norm, i.e., for any $B \in \stackrel{\circ}{\mathbb{B}}_{K}$ there exists a unique linear operator $f_{B}^{\prime}: \mathcal{V} \rightarrow C\left([0, T] ; L^{2}\left(\mathbb{R}^{6}\right)\right)$ such that

$$
\begin{gathered}
\forall \varepsilon>0 \exists \delta>0 \forall H \in \mathcal{V} \text { with }\|H\|_{\mathcal{V}}<\delta: \\
B+H \in \stackrel{\circ}{\mathbb{B}}_{K} \quad \text { and } \quad \frac{\left\|f_{B+H}-f_{B}-f_{B}^{\prime}[H]\right\|_{C\left([0, T] ; L^{2}\right)}}{\|H\|_{\mathcal{V}}}<\varepsilon .
\end{gathered}
$$

The Fréchet derivative is given by

$$
f_{B}^{\prime}[H]=f_{B}^{H}, \quad H \in \mathcal{V} .
$$

(c) For all $B, H \in \stackrel{\circ}{B}_{K}$, the solution $f_{B}^{H}$ depends Hölder-continuously on $B$ in such a way that there exists some constant $C>0$ depending only on $\stackrel{f}{f}, T, K$ and $\beta$ such that

$$
\sup _{\|H\|_{\mathcal{V}} \leq 1}\left\|f_{A}^{\prime}[H]-f_{B}^{\prime}[H]\right\|_{L^{2}\left(0, T ; L^{2}\right)} \leq C\|A-B\|_{L^{2}\left(0, T ; W^{2, \beta}\right)}^{\gamma}, A, B \in \stackrel{\circ}{\mathbb{B}}_{K}
$$

Comment As $K>0$ was arbitrary the obove results hold true on $\stackrel{\circ}{B}_{2 K}$ instead of $\stackrel{\circ}{\mathbb{B}}_{K}$. Hence they are especially true for $B \in \mathbb{B}_{K}$. 
Proof Let $C$ denote some generic positive constant depending only on $f, K, T$ and $\beta$. First note that the system (5.12) is of the type (5.1) where the quantities correspond in the following way:

\begin{tabular}{cccl} 
System (5.1) & \multicolumn{3}{c}{ System (5.12) } \\
\hline$r_{0}$ & $\widehat{=}$ & $R$ & $\geq 0$ \\
$r_{1}$ & $\widehat{=}$ & 0 & $\geq 0$ \\
$r_{2}$ & $\widehat{=}$ & $R$ & $>r_{1}$ \\
$\mathbf{a}$ & $\widehat{=}$ & 0 & $\in C\left([0, T] ; C_{b}^{1}\right)$ \\
$\mathbf{b}$ & $\widehat{=}$ & $-(v \times H) \cdot \partial_{v} f_{B}$ & $\in L^{2}\left(0, T ; C_{b} \cap H^{1}\right)$ \\
$\stackrel{\circ}{\mathbf{f}}$ & $\widehat{=}$ & 0 & $\in C_{c}^{2}\left(\mathbb{R}^{6}\right)$ \\
$\mathbf{A}$ & $\widehat{=}$ & $-\partial_{x} \psi_{f_{B}}$ & $\in C\left([0, T] ; C^{1, \gamma}\right)$ \\
$\mathbf{B}$ & $\widehat{=}$ & $B$ & $\in L^{2}\left(0, T ; C^{1, \gamma}\right)$ \\
$\mathbf{C}$ & $\widehat{=}$ & $\partial_{v} f_{B}$ & $\in L^{2}\left(0, T ; H^{1} \cap C_{b}\right)$ \\
$\boldsymbol{\chi}$ & $\widehat{=}$ & 0 & $\in C_{c}^{1}\left(\mathbb{R}^{6}\right)$
\end{tabular}

This means that the coefficients of (5.12) satisfy the regularity conditions (5.10) of Corollary 26. Hence (5.12) has a strong solution $f_{B}^{H} \in L^{\infty} \cap H^{1}(] 0, T\left[\times \mathbb{R}^{6}\right)$.

To prove Fréchet differentiability of the field-state operator we must consider the difference $f_{B+H}-f_{B}$ with $B \in \stackrel{\circ}{\mathbb{B}}_{K}$ and $H \in \mathcal{V}$ such that $B+H \in \stackrel{\circ}{\mathbb{B}}_{K}$. Therefore we will assume that $\|H\|_{\mathcal{V}}<\delta$ for some sufficiently small $\delta>0$. Now we expand the nonlinear terms in the Vlasov equation (3.4) to pick out the linear parts. We have

$$
\begin{aligned}
& \partial_{x} \psi_{f_{B+H}} \cdot \partial_{v} f_{B+H}-\partial_{x} \psi_{f_{B}} \cdot \partial_{v} f_{B} \\
& \quad=\partial_{x} \psi_{f_{B}} \cdot \partial_{v}\left(f_{B+H}-f_{B}\right)+\partial_{x} \psi_{\left(f_{B+H}-f_{B}\right)} \cdot \partial_{v} f_{B}+\mathcal{R}_{1}, \\
& \quad(v \times(B+H)) \cdot \partial_{v} f_{B+H}-(v \times B) \cdot \partial_{v} f_{B} \\
& \quad=(v \times B) \cdot \partial_{v}\left(f_{B+H}-f_{B}\right)+(v \times H) \cdot \partial_{v} f_{B}+\mathcal{R}_{2}
\end{aligned}
$$

where

$$
\mathcal{R}_{1}:=\partial_{x} \psi_{\left(f_{B+H}-f_{B}\right)} \cdot \partial_{v}\left(f_{B+H}-f_{B}\right), \quad \mathcal{R}_{2}:=(v \times H) \cdot \partial_{v}\left(f_{B+H}-f_{B}\right)
$$

are nonlinear remainders. Then $\mathcal{R}:=\mathcal{R}_{1}-\mathcal{R}_{2} \in L^{2}\left(0, T ; H^{1} \cap C_{b}\right)$ and Corollary 21 implies that

$$
\begin{aligned}
\|\mathcal{R}\|_{L^{2}} & \leq C\left\|\partial_{v} f_{B+H}-\partial_{v} f_{B}\right\|_{L^{\infty}\left(0, T ; L^{\infty}\right)}\left(\left\|\partial_{x} \psi_{f_{B+H}-f_{B}}\right\|_{L^{2}\left(0, T ; L^{2}\right)}+\|H\|_{L^{2}\left(0, T ; L^{2}\right)}\right) \\
& \leq C\left\|\partial_{v} f_{B+H}-\partial_{v} f_{B}\right\|_{L^{\infty}\left(0, T ; L^{\infty}\right)}\left(\left\|f_{B+H}-f_{B}\right\|_{L^{2}\left(0, T ; L^{2}\right)}+\|H\|_{L^{2}\left(0, T ; L^{2}\right)}\right) \\
& \leq C\|H\|_{\mathcal{V}}^{1+\gamma} .
\end{aligned}
$$

Obviously $f_{B+H}-f_{B}$ solves the initial value problem

$$
\left\{\begin{array}{l}
\partial_{t} f+v \cdot \partial_{x} f-\partial_{x} \psi_{f_{B}} \cdot \partial_{v} f-\partial_{x} \psi_{f} \cdot \partial_{v} f_{B}+(v \times B) \cdot \partial_{v} f+(v \times H) \cdot \partial_{v} f_{B}=\mathcal{R} \\
\left.f\right|_{t=0}=0
\end{array}\right.
$$

almost everywhere on $[0, T] \times \mathbb{R}^{6}$. 
From Corollary 26 (a) we know that this solution is unique. Also according to Corollary 26 (a) the system

$$
\left\{\begin{array}{l}
\partial_{t} f+v \cdot \partial_{x} f-\partial_{x} \psi_{f_{B}} \cdot \partial_{v} f-\partial_{x} \psi_{f} \cdot \partial_{v} f_{B}+(v \times B) \cdot \partial_{v} f=\mathcal{R} \\
\left.f\right|_{t=0}=0
\end{array}\right.
$$

has a unique strong solution $f_{\mathcal{R}}$. Then $f_{B}^{H}+f_{\mathcal{R}}$ is a solution of (5.13) due to linearity and thus

$$
f_{B+H}-f_{B}=f_{B}^{H}+f_{\mathcal{R}}
$$

because of uniqueness. It holds that

$$
\begin{aligned}
& \left\|f_{\mathcal{R}}(t)\right\|_{L^{2}}^{2}=2 \int_{0}^{t} \int f_{\mathcal{R}}(s) \partial_{t} f_{\mathcal{R}}(s) \mathrm{d} z \mathrm{~d} s \\
& =-2 \int_{0}^{t} \int f_{\mathcal{R}}\left(v \cdot \partial_{x} f_{\mathcal{R}}-\partial_{x} \psi_{f_{B}} \cdot \partial_{v} f_{\mathcal{R}}-\partial_{x} \psi_{f_{\mathcal{R}}} \cdot \partial_{v} f_{B}+(v \times B) \cdot \partial_{v} f_{\mathcal{R}}-\mathcal{R}\right) \mathrm{d} z \mathrm{~d} s \\
& =2 \int_{0}^{t} \int f_{\mathcal{R}}\left(\partial_{x} \psi_{f_{\mathcal{R}}} \cdot \partial_{v} f_{B}+\mathcal{R}\right) \mathrm{d} z \mathrm{~d} s \\
& \leq C \int_{0}^{t}\left\|f_{\mathcal{R}}(s)\right\|_{L^{2}}^{2}+\left\|f_{\mathcal{R}}(s)\right\|_{L^{2}}\|\mathcal{R}(s)\|_{L^{2}} \mathrm{~d} s
\end{aligned}
$$

Applying first the standard version and then the quadratic version of Gronwall's lemma yields

$$
\left\|f_{\mathcal{R}}(t)\right\|_{L^{2}} \leq C\|\mathcal{R}\|_{L^{2}\left(0, T ; L^{2}\right)} \leq C\|H\|_{\mathcal{V}}^{1+\gamma}
$$

Let now $\varepsilon>0$ be arbitrary. Then for all $t \in[0, T]$,

$$
\frac{\left\|f_{B+H}(t)-f_{B}(t)-f_{B}^{H}(t)\right\|_{L^{2}}}{\|H\|_{\mathcal{V}}}=\frac{\left\|f_{\mathcal{R}}(t)\right\|_{L^{2}}}{\|H\|_{\mathcal{V}}} \leq C\|H\|_{\mathcal{V}}^{\gamma}<\varepsilon
$$

if $\delta$ is sufficiently small. Since the inequality holds for all $t \in[0, T]$ and all the terms depend continuously on time this also means that

$$
\frac{\left\|f_{B+H}-f_{B}-f_{B}^{H}\right\|_{C\left([0, T] ; L^{2}\right)}}{\|H\|_{\mathcal{V}}}=\max _{t \in[0, T]} \frac{\left\|f_{B+H}(t)-f_{B}(t)-f_{B}^{H}(t)\right\|_{L^{2}}}{\|H\|_{\mathcal{V}}}<\varepsilon
$$

Hence the assertions (a) and (b) are proved and the Fréchet derivative is determined by the system (5.12).

To prove (c) let $A, B, H \in \stackrel{\circ}{\mathbb{B}}_{K}$ be arbitrary and suppose that $\|H\|_{\mathcal{V}} \leq 1$. According to Lemma $10(\mathrm{~d})$, we can choose sequences $\left(A_{k}\right),\left(B_{k}\right),\left(H_{k}\right) \subset \mathbb{M}$ such that

$$
\left\|A_{k}-A\right\|_{L^{2}\left(0, T ; W^{2, \beta}\right)} \rightarrow 0, \quad\left\|B_{k}-B\right\|_{L^{2}\left(0, T ; W^{2, \beta}\right)} \quad \rightarrow 0, \quad\left\|H_{k}-H\right\|_{L^{2}\left(0, T ; W^{2, \beta}\right)} \rightarrow 0
$$

if $k$ tends to infinity. 
From Corollary 26 we can conclude that

$$
\left\|f_{A_{k}}^{H_{k}}\right\|_{H^{1}(] 0, T\left[\times \mathbb{R}^{6}\right)} \leq C \quad \text { and } \quad\left\|f_{B_{k}}^{H_{k}}\right\|_{H^{1}(] 0, T\left[\times \mathbb{R}^{6}\right)} \leq C
$$

and also

$$
f_{A_{k}}^{H_{k}} \rightarrow f_{A}^{H} \quad \text { and } \quad f_{B_{k}}^{H_{k}} \rightarrow f_{B}^{H} \quad \text { in } H^{1}(] 0, T\left[\times \mathbb{R}^{6}\right) .
$$

Since the $(x, v)$-supports of all occurring functions are contained in some ball $B_{\varrho}(0)$ whose radius $r$ depends only on $f, K, T$ and $\beta$ but not on $k$, we can apply the RellichKondrachov theorem to obtain

$$
f_{A_{k}}^{H_{k}} \rightarrow f_{A}^{H} \quad \text { and } \quad f_{B_{k}}^{H_{k}} \rightarrow f_{B}^{H} \quad \text { in } L^{2}\left([0, T] \times \mathbb{R}^{6}\right)
$$

up to a subsequence. As $A_{k}, B_{k}$ and $H_{k}$ satisfy the regularity assumptions (5.2), $f_{A_{k}}^{H_{k}}$ and $f_{B_{k}}^{H_{k}}$ are classical solutions and can be described implicitely by the representation formula (5.7). Note that Lemma 15 holds true for $\varrho$ instead of $R$. Hence for all $s, t \in[0, T]$,

$$
\left\|Z_{F}(s, t, \cdot)\right\|_{L^{\infty}\left(B_{\varrho}(0)\right)} \leq C, \quad\left\|\partial_{z} f_{F}(s)\right\|_{\infty} \leq C, \quad\left\|D_{z}^{2} f_{F}\right\|_{L^{2}\left(0, T ; L^{2}\right)} \leq C
$$

for all $F \in\left\{A_{k}, B_{k} \mid k \in \mathbb{N}\right\}$. Also recall that we know from Lemma 16 (with $\varrho$ instead of $R$ ) that for all $s, t \in[0, T]$,

$$
\begin{gathered}
\left\|f_{A_{k}}(s)-f_{B_{k}}(s)\right\|_{\infty} \leq C\left\|A_{k}-B_{k}\right\|_{L^{2}\left(0, T ; W^{2, \beta}\right)}, \\
\left\|\partial_{z} f_{A_{k}}(s)-\partial_{z} f_{B_{k}}(s)\right\|_{\infty} \leq C\left\|A_{k}-B_{k}\right\|_{L^{2}\left(0, T ; W^{2, \beta}\right)}^{\gamma}, \\
\left\|Z_{A_{k}}(s, t, \cdot)-Z_{B_{k}}(s, t, \cdot)\right\|_{L^{\infty}\left(B_{\varrho}(0)\right)} \leq C\left\|A_{k}-B_{k}\right\|_{L^{2}\left(0, T ; W^{2, \beta}\right)} .
\end{gathered}
$$

Then it holds that

$$
\begin{aligned}
& \left\|f_{A_{k}}^{H_{k}}(t)-f_{B_{k}}^{H_{k}}(t)\right\|_{L^{2}} \\
& \leq \int_{0}^{t}\left\|\left(\partial_{x} \psi_{f_{A_{k}}^{H_{k}}} \cdot \partial_{v} f_{A_{k}}\right)\left(s, Z_{A_{k}}(s, t, \cdot)\right)-\left(\partial_{x} \psi_{f_{B_{k}}^{H_{k}}} \cdot \partial_{v} f_{B_{k}}\right)\left(s, Z_{B_{k}}(s, t, \cdot)\right)\right\|_{L^{2}} \mathrm{~d} s \\
& \quad+\int_{0}^{t}\left\|\left(V_{A_{k}} \times H_{k}\left(s, X_{A_{k}}\right)\right) \cdot \partial_{v} f_{A_{k}}\left(s, Z_{A_{k}}\right)-\left(V_{B_{k}} \times H_{k}\left(s, X_{B_{k}}\right)\right) \cdot \partial_{v} f_{B_{k}}\left(s, Z_{B_{k}}\right)\right\|_{L^{2}} \mathrm{~d} s \\
& \leq C \int_{0}^{t}\left(1+\left\|D_{z}^{2} f_{A_{k}}(s)\right\|_{L^{2}}+\left\|H_{k}(s)\right\|_{W^{2, \beta}}\right)\left\|Z_{A_{k}}(s)-Z_{B_{k}}(s)\right\|_{L^{\infty}\left(B_{\varrho}(0)\right)} \mathrm{d} s \\
& \quad+C \int_{0}^{t}\left(1+\left\|H_{k}(s)\right\|_{W^{2, \beta}}\right)\left\|\partial_{v} f_{A_{k}}(s)-\partial_{v} f_{B_{k}}(s)\right\|_{L^{\infty} \mathrm{d} s} \\
& \quad+C \int_{0}^{t}\left\|f_{A_{k}}^{H_{k}}(s)-f_{B_{k}}^{H_{k}}(s)\right\|_{L^{2}} \mathrm{~d} s
\end{aligned}
$$

and thus by Gronwall's lemma,

$$
\left\|f_{A_{k}}^{H_{k}}-f_{B_{k}}^{H_{k}}\right\|_{L^{2}\left(0, T ; L^{2}\right)} \leq C\left\|f_{A_{k}}^{H_{k}}-f_{B_{k}}^{H_{k}}\right\|_{L^{\infty}\left(0, T ; L^{2}\right)} \leq C\left\|A_{k}-B_{k}\right\|_{L^{2}\left(0, T ; W^{2, \beta}\right)}^{\gamma} .
$$


If $k \rightarrow \infty$ we obtain

$$
\left\|f_{A}^{H}-f_{B}^{H}\right\|_{L^{2}\left(0, T ; L^{2}\right)} \leq C\|A-B\|_{L^{2}\left(0, T ; W^{2, \beta}\right)}^{\gamma}
$$

that is (c). 


\section{Chapter 6}

\section{The tracking problem}

\subsection{Optimal control with $\mathbb{B}_{K}$-fields}

\subsubsection{The model}

We will now consider the Vlasov-Poisson system equipped with an external magnetic field $B \in \mathbb{B}_{K}$ on the time interval $[0, T]$ with initial state $f \in C_{c}^{2}\left(\mathbb{R}^{6}\right)$. Our aim is to control the time evolution of the distribution function $f=f(t, x, v)$ in such a way that its value at time $T$ matches a desired distribution function $f_{d} \in C_{c}^{2}\left(\mathbb{R}^{6}\right)$ as closely as possible. More precisely we want to find a magnetic field $B$ such that the $L^{2}$-difference $\left\|f(T)-f_{d}\right\|_{L^{2}}$ becomes as small as possible. Therefore we intend to minimze the cost functional

$$
I(f, B)=\frac{1}{2}\left\|f(T)-f_{d}\right\|_{L^{2}\left(\mathbb{R}^{6}\right)}^{2}+\frac{\lambda}{2}\left\|D_{x} B\right\|_{L^{2}\left([0, T] \times \mathbb{R}^{3} ; \mathbb{R}^{3}\right)}^{2}
$$

where $\lambda$ is a nonnegative parameter. In this section the field $B$ is the control in our model and thus the field-state operator can also be referred to as the control-state operator. Since $\|f(t)\|_{p}=\|\stackrel{\circ}{f}\|_{p}$ for all $1 \leq p \leq \infty, t \in[0, T]$ it makes sense to choose $f_{d}$ in such a way that $\left\|f_{d}\right\|_{p}=\left\|f^{\circ}\right\|_{p}$ for all $1 \leq p \leq \infty$ because otherwise the exact matching $f(T)=f_{d}$ would be impossible from the outset. Note that the $L^{2}$-Norm of the functional matrix is given by

$$
\left\|D_{x} B\right\|_{L^{2}}^{2}=\sum_{i=1}^{3}\left\|\partial_{x_{i}} B\right\|_{L^{2}}^{2}=\sum_{i=1}^{3} \sum_{j=1}^{3}\left\|\partial_{x_{i}} B_{j}\right\|_{L^{2}}^{2} .
$$

We will also use the notation

$$
\left\langle D_{x} B, D_{x} H\right\rangle_{L^{2}}=\sum_{i=1}^{3}\left\langle\partial_{x_{i}} B, \partial_{x_{i}} H\right\rangle_{L^{2}}=\sum_{i=1}^{3} \sum_{j=1}^{3}\left\langle\partial_{x_{i}} B_{j}, \partial_{x_{i}} H_{j}\right\rangle_{L^{2}} .
$$

At first appearance it seems that the term $\frac{\lambda}{2}\left\|D_{x} B\right\|_{L^{2}}^{2}$ is useless or even counterproductive as we actually want to minimize the expression $\left\|f(T)-f_{d}\right\|_{L^{2}}$. However this term grants some crucial advantages in terms of variational calculus if $\lambda>0$. In this case a magnetic field is "punished" by high values of the cost functional if its derivatives become large. Of course the amount of punishment depends on the size of $\lambda$. Thus the 
term has a smoothing effect on the optimal magnetic field and is hence referred to as the regularization term. Now the optimization problem is to minimize the functional $I$ under the following constraints:

- $B$ is an admissible field, i.e., $B \in \mathbb{B}_{K}$

- $f$ is a strong solution of the Vlasov-Poisson system

$$
\partial_{t} f+v \cdot \partial_{x} f-\partial_{x} \psi_{f} \cdot \partial_{v} f+(v \times B) \cdot \partial_{v} f=0,\left.\quad f\right|_{t=0}=\stackrel{\circ}{f}
$$

to the control $B$.

Recalling the definition of the control-state operator we can alternatively consider the optimization problem

$$
\begin{aligned}
\text { Minimize } & J(B)=\frac{1}{2}\left\|f_{B}(T)-f_{d}\right\|_{L^{2}}^{2}+\frac{\lambda}{2}\left\|D_{x} B\right\|_{L^{2}\left([0, T] \times \mathbb{R}^{3}\right)}^{2} \\
\text { s.t. } & B \in \mathbb{B}_{K}
\end{aligned}
$$

which is equivalent to the first one. The advantage is that the cost functional $J$ depends only on $B$ as the side condition is implemented by the control-state operator.

\subsubsection{Existence of a globally optimal solution}

Of course such an optimization problem does only make sense if there actually exists at least one globally optimal solution. This fact will be established in the next Theorem. The proof is quite short as most of the work was already done in the Chapters 3 and 4 .

Theorem 28 The optimization problem (6.2) possesses a globally optimal solution $\bar{B}$, i.e., for all $B \in \mathbb{B}_{K}, J(\bar{B}) \leq J(B)$. Then $\bar{B}$ is also called the optimal control and $f_{\bar{B}}$ is called its optimal state.

Proof Suppose that $\lambda>0$ (if $\lambda=0$ the proof is similar but even easier). The cost functional $J$ is bounded from below since $J(B) \geq 0$ for all $B \in \mathbb{B}_{K}$. Hence $M:=\inf _{B \in \mathbb{B}_{K}} J(B)$ exists and there also exists a minimizing sequence $\left(B_{k}\right)_{k \in \mathbb{N}}$ such that $J\left(B_{k}\right) \rightarrow M$ if $k \rightarrow \infty$. As $\mathbb{B}_{K} \subset \mathcal{V}$ is weakly compact according to Lemma 10 it holds that $B_{k} \rightarrow \bar{B}$ in $\mathcal{V}$ for some weak limit $\bar{B} \in \mathbb{B}_{K}$ after extraction of a subsequence. Then Proposition 22 yields $f_{B_{k}} \rightarrow f_{\bar{B}}$ in $W^{1,2}\left(0, T ; L^{2}\right)$ after subsequence extraction. Now for any $\varphi \in L^{2}\left(\mathbb{R}^{6}\right)$,

$$
\begin{aligned}
& \int\left(f_{B_{k}}(T, z)-f_{\bar{B}}(T, z)\right) \varphi(z) \mathrm{d} z=\int_{0}^{T} \frac{\mathrm{d}}{\mathrm{d} t} \int\left(f_{B_{k}}(t, z)-f_{\bar{B}}(t, z)\right) \varphi(z) \mathrm{d} z \mathrm{~d} t \\
& =\int_{0}^{T} \int\left(\partial_{t} f_{B_{k}}(t, z)-\partial_{t} f_{\bar{B}}(t, z)\right) \varphi(z) \mathrm{d} z \mathrm{~d} t \rightarrow 0, \quad k \rightarrow \infty
\end{aligned}
$$

which means that $f_{B_{k}}(T) \rightarrow f_{\bar{B}}(T)$ in $L^{2}\left(\mathbb{R}^{6}\right)$. Together with the weak lower semicontinuity of the $L^{2}$-norm this implies that 


$$
\begin{aligned}
J(\bar{B}) & =\frac{1}{2}\left\|f_{\bar{B}}(T)-f_{d}\right\|_{L^{2}}^{2}+\frac{\lambda}{2}\left\|D_{x} \bar{B}\right\|_{L^{2}}^{2} \\
& \leq \liminf _{k \rightarrow \infty}\left[\frac{1}{2}\left\|f_{B_{k}}(T)-f_{d}\right\|_{L^{2}}^{2}\right]+\liminf _{k \rightarrow \infty}\left[\frac{\lambda}{2}\left\|D_{x} B_{k}\right\|_{L^{2}}^{2}\right] \\
& \leq \liminf _{k \rightarrow \infty}\left[\frac{1}{2}\left\|f_{B_{k}}(T)-f_{d}\right\|_{L^{2}}^{2}+\frac{\lambda}{2}\left\|D_{x} B_{k}\right\|_{L^{2}}^{2}\right]=\lim _{k \rightarrow \infty} J\left(B_{k}\right)=M .
\end{aligned}
$$

By the definition of infimum this yields $J(\bar{B})=M$.

Of course this theorem does not provide uniqueness of a globally optimal solution.

\subsubsection{Necessary conditions for local optimality}

Since the control-state operator $f$. is nonlinear we cannot expect $J$ to be convex. Of course the regularization term is strictly convex with respect to $B$ if $\lambda>0$ but if $\lambda$ is rather small (which makes sense in this model) there is no chance that this property can be transferred to $J$. For that reason it is possible that $J$ has more than one locally optimal solution. They are defined as follows:

Definition $29 A$ control $\bar{B} \in \mathbb{B}_{K}$ is called a locally optimal solution of the optimization problem (6.2) iff there exists $\delta>0$ such that

$$
J(\bar{B}) \leq J(B) \quad \text { for all } \quad B \in B_{\delta}(\bar{B}) \cap \mathbb{B}_{K}
$$

where $B_{\delta}(\bar{B})$ is the open ball in $L^{2}\left(0, T ; W^{2, \beta}\left(\mathbb{R}^{3} ; \mathbb{R}^{3}\right)\right)$ with radius $\delta$ and center $\bar{B}$.

To get an idea of necessary conditions let us at first consider some differentiable function $\varphi: \mathbb{R}^{d} \rightarrow \mathbb{R}$, let $U$ be any convex open subset of $\mathbb{R}^{d}$ and suppose that $\left.\varphi\right|_{\bar{U}}$ has a local minimum at the point $x \in \bar{U}$. For all $h \in \mathbb{R}^{d}$ with $x+h \in \bar{U}$ we have $x+t h \in \bar{U}$ for all $t \in[0,1]$ because of convexity. Then the function $[0,1] \ni t \mapsto \varphi(x+t h)$ is differentiable with

$$
0 \leq \lim _{\substack{t \rightarrow 0 \\ \geq}} \frac{\varphi(x+t h)-\varphi(x)}{t}=\left.\frac{\mathrm{d}}{\mathrm{d} t} \varphi(x+t h)\right|_{t=0}=\nabla \varphi(x) \cdot h .
$$

Of course $\nabla \varphi(x)=0$ if $x \in U$. This fact can be generalized to functionals on Banach spaces if the total gradient is replaced by the Fréchet derivative. Therefore we can establish the following necessary optimality condition: 
Lemma 30 The cost functional $J$ is Fréchet differentiable on $\mathbb{B}_{K}$ with Fréchet derivative

$$
J^{\prime}(B)[H]=\left\langle f_{B}(T)-f_{d}, f_{B}^{\prime}(T)[H]\right\rangle_{L^{2}\left(\mathbb{R}^{6}\right)}+\lambda\left\langle D_{x} B, D_{x} H\right\rangle_{L^{2}\left([0, T] \times \mathbb{R}^{3} ; \mathbb{R}^{3 \times 3}\right)}, \quad H \in \mathcal{V} .
$$

Let $\bar{B} \in \mathbb{B}_{K}$ be a locally optimal solution of the optimization problem (6.2). Then

$$
J^{\prime}(\bar{B})[H]\left\{\begin{array}{ll}
=0, & \text { if } \bar{B} \in \mathbb{B}_{K} \\
\geq 0, & \text { if } \bar{B} \in \partial \mathbb{B}_{K}
\end{array}, \quad H \in \mathcal{V} \text { with } \bar{B}+H \in \mathbb{B}_{K}\right.
$$

Proof As the control-state operator is Fréchet differentiable on $\mathbb{B}_{K}$ so is the cost functional $J$ by chain rule. If $\bar{B}+H \in \mathbb{B}_{K}$ the function $[0,1] \ni t \mapsto J(B+t H) \in \mathbb{R}$ is differentiable with respect to $t$ and since $\bar{B}$ is a local minimizer,

$$
0 \leq\left.\frac{\mathrm{d}}{\mathrm{d} t} J(\bar{B}+t H)\right|_{t=0}=\left.\left(J^{\prime}(\bar{B}+t H)\left[\frac{\mathrm{d}}{\mathrm{d} t}(\bar{B}+t H)\right]\right)\right|_{t=0}=J^{\prime}(\bar{B})[H]
$$

for any $H \in \mathcal{V}$ with $B+H \in \mathbb{B}_{K}$.

If we consider $\mathbb{B}_{K}$ as a subset of $L^{2}\left([0, T] \times \mathbb{R}^{3} ; \mathbb{R}^{3}\right)$ it might be possible to find an adjoint operator $\left(f_{B}^{\prime}(T)\right)^{*}$ of $f_{B}^{\prime}(T)$ such that

$$
\begin{aligned}
J^{\prime}(B)[H] & =\left\langle f_{B}(T)-f_{d}, f_{B}^{\prime}(T)[H]\right\rangle_{L^{2}\left(\mathbb{R}^{6}\right)}+\lambda \sum_{i=1}^{3}\left\langle\partial_{x_{i}} B, \partial_{x_{i}} H\right\rangle_{L^{2}\left([0, T] \times \mathbb{R}^{3}\right)} \\
& =\left\langle\left(f_{B}^{\prime}(T)\right)^{*}\left[f_{B}(T)-f_{d}\right], H\right\rangle_{L^{2}\left([0, T] \times \mathbb{R}^{3}\right)}-\lambda \sum_{i=1}^{3}\left\langle\partial_{x_{i}}^{2} B, H\right\rangle_{L^{2}\left([0, T] \times \mathbb{R}^{3}\right)} \\
& =\left\langle\left(f_{B}^{\prime}(T)\right)^{*}\left[f_{B}(T)-f_{d}\right]-\lambda \Delta_{x} B, H\right\rangle_{L^{2}\left([0, T] \times \mathbb{R}^{3}\right)}, \quad H \in \mathbb{B}_{K} .
\end{aligned}
$$

This means that $J^{\prime}$ has the explicit description

$$
J^{\prime}(B)=\left(f_{B}^{\prime}(T)\right)^{*}\left[f_{B}(T)-f_{d}\right]-\lambda \Delta_{x} B .
$$

If now $\bar{B} \in \operatorname{int} \mathbb{B}_{K}$ is a locally optimal solution it satisfies the semilinear Poisson equation

$$
-\Delta_{x} B=-\frac{1}{\lambda}\left(f_{B}^{\prime}(T)\right)^{*}\left[f_{B}(T)-f_{d}\right] .
$$

In general such an adjoint operator is not uniquely determined. This means that we cannot deduce uniqueness of our optimal solution. A common technique to find an adjoint operator is the Lagrangian technique. For $B \in \mathcal{V}$ and $f, g \in H^{1}(] 0, T\left[\times \mathbb{R}^{6}\right)$ with supp $f(t) \subset B_{R}(0)$ for all $t \in[0, T]$ we define

$$
\begin{aligned}
\mathcal{L}(f, B, g) & :=I(f, B)-\int_{[0, T] \times \mathbb{R}^{6}}\left(\partial_{t} f+v \cdot \partial_{x} f-\partial_{x} \psi_{f} \cdot \partial_{v} f+(v \times B) \cdot \partial_{v} f\right) g \mathrm{~d}(t, x, v) \\
= & \frac{1}{2}\left\|f(T)-f_{d}\right\|_{L^{2}}+\frac{\lambda}{2}\left\|D_{x} B\right\|_{L^{2}}^{2} \\
& \quad-\int_{[0, T] \times \mathbb{R}^{6}}\left(\partial_{t} f+v \cdot \partial_{x} f-\partial_{x} \psi_{f} \cdot \partial_{v} f+(v \times B) \cdot \partial_{v} f\right) g \mathrm{~d}(t, x, v) .
\end{aligned}
$$


$\mathcal{L}$ is called the Lagrangian. Obviously by integration by parts,

$$
\begin{aligned}
\mathcal{L}(f, B, g)=\frac{1}{2}\left\|f(T)-f_{d}\right\|_{L^{2}}+\frac{\lambda}{2}\left\|D_{x} B\right\|_{L^{2}}^{2}+\langle g(0), f(0)\rangle_{L^{2}}-\langle g(T), f(T)\rangle_{L^{2}} \\
+\int_{[0, T] \times \mathbb{R}^{6}}\left(\partial_{t} g+v \cdot \partial_{x} g-\partial_{x} \psi_{f} \cdot \partial_{v} g+(v \times B) \cdot \partial_{v} g\right) f \mathrm{~d}(t, x, v) .
\end{aligned}
$$

In the definition of the Lagrangian $f, B$ and $g$ are independent functions. However inserting $f=f_{B}$ yields

$$
J(B)=\mathcal{L}\left(f_{B}, B, g\right), \quad B \in \mathbb{B}_{K}, g \in H^{1}(] 0, T\left[\times \mathbb{R}^{6}\right) .
$$

It is important that this equality does not depend on the choice of $g$. Since $\mathcal{L}$ is Fréchet differentiable with respect to $f$ in the $H^{1}(] 0, T\left[\times \mathbb{R}^{6}\right)$-sense and with respect to $B$ in the $L^{2}\left(0, T ; W^{2, \beta}\right)$-sense we can use this fact to compute the derivative of $J$ alternatively. By chain rule,

$$
J^{\prime}(B)[H]=\left(\partial_{f} \mathcal{L}\right)\left(f_{B}, B, g\right)\left[f_{B}^{\prime}[H]\right]+\left(\partial_{B} \mathcal{L}\right)\left(f_{B}, B, g\right)[H], \quad B \in \mathbb{B}_{K}, H \in \mathcal{V}
$$

for any $g \in H^{1}(] 0, T\left[\times \mathbb{R}^{6}\right)$. Here $\partial_{f} \mathcal{L}$ and $\partial_{B} \mathcal{L}$ denote the partial Fréchet derivative of $\mathcal{L}$ with respect to $f$ and $B$. We will now fix $f, g$ and $B$. Then

$$
\begin{aligned}
\left(\partial_{f} \mathcal{L}\right)(f, B, g)[h]= & \left\langle f(T)-f_{d}, h(T)\right\rangle_{L^{2}}-\langle g(T), h(T)\rangle_{L^{2}}+\langle g(0), h(0)\rangle_{L^{2}} \\
& +\int_{[0, T] \times \mathbb{R}^{6}}\left(\partial_{t} g+v \cdot \partial_{x} g-\partial_{x} \psi_{f} \cdot \partial_{v} g+(v \times B) \cdot \partial_{v} g\right) h \mathrm{~d}(t, x, v) \\
& +\int_{[0, T] \times \mathbb{R}^{6}} \partial_{x} \psi_{h} \cdot \partial_{v} f g \mathrm{~d}(t, x, v) \\
= & \left\langle f(T)-f_{d}, h(T)\right\rangle_{L^{2}}-\langle g(T), h(T)\rangle_{L^{2}}+\langle g(0), h(0)\rangle_{L^{2}} \\
& +\int_{[0, T] \times \mathbb{R}^{6}}\left(\partial_{t} g+v \cdot \partial_{x} g-\partial_{x} \psi_{f} \cdot \partial_{v} g+(v \times B) \cdot \partial_{v} g\right) h \mathrm{~d}(t, x, v) \\
& -\int_{[0, T] \times \mathbb{R}^{6}} \Phi_{f, g}(t, x, v) h \mathrm{~d}(t, x, v)
\end{aligned}
$$

for all $h \in H^{1}(] 0, T\left[\times \mathbb{R}^{6}\right)$ where

$$
\Phi_{f, g}(t, x)=-\int \frac{x-y}{|x-y|^{3}} \cdot \partial_{v} f(t, y, w) g(t, y, w) \mathrm{d}(y, w)
$$

as defined in (5.3) and

$$
\begin{gathered}
\left(\partial_{B} \mathcal{L}\right)(f, B, g)[H]=\lambda\left\langle D_{x} B, D_{x} H\right\rangle_{L^{2}}-\int_{[0, T] \times \mathbb{R}^{6}}(v \times H) \cdot \partial_{v} f g \mathrm{~d}(t, x, v) \\
\quad=\int_{[0, T] \times \mathbb{R}^{3}}-\lambda \Delta_{x} B \cdot H \mathrm{~d}(t, x)+\int_{[0, T] \times \mathbb{R}^{3}}\left(\int_{\mathbb{R}^{3}} v \times \partial_{v} f g \mathrm{~d} v\right) \cdot H \mathrm{~d}(t, x) .
\end{gathered}
$$

for all $H \in \mathcal{V}$. 
Apparently the derivative with respect to $B$ looks pretty nice while the derivative with respect to $f$ is rather complicated. However if we insert those terms in (6.3) we can still choose $g$. Now the idea of the Lagrangian technique is to choose $g$ in such a way that the term $\left(\partial_{f} \mathcal{L}\right)\left(f_{B}, B, g\right)\left[f_{B}^{\prime}[H]\right]$ vanishes.

We consider the following final value problem which we will call the costate equation:

$$
\left\{\begin{array}{l}
\partial_{t} g+v \cdot \partial_{x} g-\partial_{x} \psi_{f_{B}} \cdot \partial_{v} g+(v \times B) \cdot \partial_{v} g=\Phi_{f_{B}, g} \chi \\
\left.g\right|_{t=T}=f_{B}(T)-f_{d}
\end{array}\right.
$$

where $\chi \in C_{c}^{2}\left(\mathbb{R}^{6} ;[0,1]\right)$ with $\chi=1$ on $B_{R_{Z}}(0)$ and supp $\chi \in B_{2 R_{Z}}(0)$ denotes an arbitrary but fixed cut-off function. Here $R_{Z}$ is the constant from Lemma 15. Existence and uniqueness of a strong solution to this system will be established in the following theorem:

Theorem 31 Let $B \in \mathbb{B}_{K}$ be arbitrary and let $f_{B}$ be its strong solution as given by the control-state operator. Then the costate equation (6.6) possesses a unique strong solution $g_{B} \in W^{1,2}\left(0, T ; C_{b}\left(\mathbb{R}^{6}\right)\right) \cap C\left([0, T] ; C_{b}^{1}\left(\mathbb{R}^{6}\right)\right) \cap L^{\infty}\left(0, T ; H^{2}\left(\mathbb{R}^{6}\right)\right)$ with compact support $\operatorname{supp} g_{B}(t) \subset B_{R^{*}}(0)$ for all $t \in[0, T]$ and some radius $R^{*}>0$ depending only on $\stackrel{\circ}{f}, f_{d}, T, K$ and $\beta$.

In this case $\left.g_{B}\right|_{B_{R}(0)}$ does not depend on the choice of $\chi$ as long as $\chi=1$ on $B_{R_{Z}}(0)$.

Moreover $g_{B}$ depends Hölder-continuously on $B$ in such a way that there exists some constant $C \geq 0$ depending only on $\stackrel{f}{f} f_{d}, T, K, \beta$ and $\|\chi\|_{C_{b}^{1}}$ such that

$$
\left\|g_{B}-g_{H}\right\|_{W^{1,2}\left(0, T ; C_{b}\right)}+\left\|g_{B}-g_{H}\right\|_{C\left([0, T] ; C_{b}^{1}\right)} \leq C\|B-H\|_{L^{2}\left(0, T ; W^{2, \beta}\right)}^{\gamma}, \quad B, H \in \mathbb{B}_{K} .
$$

The very technical proof is outsourced to the appendix.

Now inserting the state $f_{B}$ and its costate $g_{B}$ in (6.3) yields

$$
J^{\prime}(B)[H]=\left(\partial_{B} \mathcal{L}\right)\left(f_{B}, B, g_{B}\right)[H], \quad H \in \mathcal{V}
$$

since $\left.f_{B}^{\prime}[H]\right|_{t=0}=0$. This provides a necessary optimality condition:

\section{Theorem 32}

(a) The Fréchet derivative of $J$ at the point $B \in \mathbb{B}_{K}$ is given by

$$
J^{\prime}(B)[H]=\int_{[0, T] \times \mathbb{R}^{3}}\left(-\lambda \Delta_{x} B+\int_{\mathbb{R}^{3}} v \times \partial_{v} f_{B} g_{B} \mathrm{~d} v\right) \cdot H \mathrm{~d}(t, x), H \in \mathcal{V} .
$$

(b) Let us assume that $\bar{B} \in \mathbb{B}_{K}$ is a locally optimal solution of the optimization problem (6.2). Then for all $B \in \mathbb{B}_{K}$,

$$
\int_{[0, T] \times \mathbb{R}^{3}}\left(-\lambda \Delta_{x} \bar{B}+\int_{\mathbb{R}^{3}} v \times \partial_{v} f_{\bar{B}} g_{\bar{B}} \mathrm{~d} v\right) \cdot(B-\bar{B}) \mathrm{d}(t, x)=\left\{\begin{array}{ll}
=0, & \text { if } \bar{B} \in \mathbb{B}_{K} \\
\geq 0, & \text { if } \bar{B} \in \partial \mathbb{B}_{K}
\end{array} .\right.
$$


(c) If we additionally assume that $\bar{B} \in \stackrel{\circ}{\mathbb{B}}_{K}$ then $\bar{B}$ satisfies the semilinear Poisson equation

$$
-\Delta_{x} \bar{B}=-\frac{1}{\lambda} \int_{\mathbb{R}^{3}} v \times \partial_{v} f_{\bar{B}} g_{\bar{B}} \mathrm{~d} v .
$$

In this case $\bar{B} \in C\left([0, T] ; C_{b}^{2}\left(\mathbb{R}^{3}\right)\right)$ with

$$
\bar{B}(t, x)=-\frac{1}{4 \pi \lambda} \iint \frac{1}{|x-y|} w \times \partial_{v} f_{\bar{B}}(t, y, w) g_{\bar{B}}(t, y, w) \mathrm{d}(y, w)
$$

for all $t \in[0, T]$ and $x \in \mathbb{R}^{3}$. Thus $\bar{B}$ does not depend on the choice of $\chi$ as long as $\chi=1$ on $B_{R_{Z}}(0)$ as it only depends on $\left.g_{\bar{B}}\right|_{B_{R}(0)}$.

Proof (a) follows immediately from (6.5) and (6.7). Then (b) is a direct consequence of Lemma 30 and (a) with $H:=B-\bar{B}$. Now, (b) implies (6.8) and then (6.9) follows from Lemma 6 . We must still prove that $\bar{B} \in C\left([0, T] ; C_{b}^{2}\left(\mathbb{R}^{3}\right)\right)$. First note that $f_{\bar{B}}, g_{\bar{B}} \in W^{1,2}\left(0, T ; C_{b}\left(\mathbb{R}^{6}\right)\right) \cap C\left([0, T] ; C_{b}^{1}\left(\mathbb{R}^{6}\right)\right)$ since $\bar{B} \in \mathbb{B}_{K}$. Hence

$$
p:[0, T] \times \mathbb{R}^{3} \rightarrow \mathbb{R}^{3}, \quad(t, x) \mapsto \int_{\mathbb{R}^{3}} v \times \partial_{v} f_{\bar{B}} g_{\bar{B}} \mathrm{~d} v
$$

is continuous. Let $\left(f_{k}\right)_{k \in \mathbb{N}} \in C\left([0, T] ; C_{b}^{2}\left(\mathbb{R}^{6}\right)\right)$ be a sequence with supp $f_{k}(t) \subset B_{R}(0)$ for all $t \in[0, T]$ and $k \in \mathbb{N}$ and $f_{k} \rightarrow f_{\bar{B}}$ in $C\left([0, T] ; C_{b}^{1}\right)$ if $k \rightarrow \infty$. For any $k \in \mathbb{N}$ let $p_{k}$ be defined just as $p$ but with $f_{k}$ instead of $f_{\bar{B}}$. Then for any $i \in\{1,2,3\}, p_{k}$ is continuously partially differentiable with respect to $x_{i}$ with

$$
\begin{aligned}
& \partial_{x_{i}} p_{k}=\int_{\mathbb{R}^{3}}\left(v \times \partial_{v} f_{k}\right) \partial_{x_{i}} g_{\bar{B}}+\partial_{x_{i}}\left(v \times \partial_{v} f_{k}\right) g_{\bar{B}} \mathrm{~d} v \\
& =\int_{\mathbb{R}^{3}}\left(v \times \partial_{v} f_{k}\right) \partial_{x_{i}} g_{\bar{B}}+\left(v \times \partial_{v} \partial_{x_{i}} f_{k}\right) g_{\bar{B}} \mathrm{~d} v=\int_{\mathbb{R}^{3}}\left(v \times \partial_{v} f_{k}\right) \partial_{x_{i}} g_{\bar{B}}-\left(v \times \partial_{v} g_{\bar{B}}\right) \partial_{x_{i}} f_{k} \mathrm{~d} v \\
& \rightarrow \int_{\mathbb{R}^{3}}\left(v \times \partial_{v} f_{\bar{B}}\right) \partial_{x_{i}} g_{\bar{B}}-\left(v \times \partial_{v} g_{\bar{B}}\right) \partial_{x_{i}} f_{\bar{B}} \mathrm{~d} v
\end{aligned}
$$

in $C\left([0, T] ; C_{b}\right)$ if $k \rightarrow \infty$. On the other hand $p_{k} \rightarrow p, k \rightarrow \infty$ in $C\left([0, T] ; C_{b}\right)$. Since $i$ was arbitrary this implies that $p \in C\left([0, T] ; C_{b}^{1}\left(\mathbb{R}^{3} ; \mathbb{R}^{3}\right)\right)$ with

$$
\partial_{x_{i}} p=-\int_{\mathbb{R}^{3}}\left(v \times \partial_{v} f_{\bar{B}}\right) \partial_{x_{i}} g_{\bar{B}}-\left(v \times \partial_{v} g_{\bar{B}}\right) \partial_{x_{i}} f_{\bar{B}} \mathrm{~d} v
$$

for any $i \in\{1,2,3\}$. Consequently $\bar{B} \in C\left([0, T] ; C_{b}^{2}\left(\mathbb{R}^{3} ; \mathbb{R}^{3}\right)\right)$. Since $g_{\bar{B}}$ does not depend on $\chi$ as long as $\chi=1$ on $B_{R_{Z}}(0)$ the same holds for $\bar{B}$.

Note that Theorem 32 provides only a necessary but not a sufficient condition for local optimality. If a control $B$ satisfies the above condition it could still be a saddle point or even a local maximum point. Theorem 32 does also not provide uniqueness of the locally optimal solution. However the globally optimal solution that is predicted by Theorem 
28 is also locally optimal. Thus we have at least one control to satisfy the necessary optimality condition of Theorem 32 .

Assuming that there exists some locally optimal solution $\bar{B} \in \mathbb{B}_{K}$ we can easily deduce from Theorem 32 that the triple $\left(f_{\bar{B}}, g_{\bar{B}}, \bar{B}\right)$ is a strong solution of some certain system of equations.

Corollary 33 Suppose that $\bar{B} \in \stackrel{\circ}{B}_{K}$ is a locally optimal solution of the optimization problem (6.2). Let $f_{\bar{B}}$ and $g_{\bar{B}}$ be its induced state and costate.

Then $f_{\bar{B}}, g_{\bar{B}} \in C^{1}\left([0, T] \times \mathbb{R}^{6}\right)$ and the triple $\left(f_{\bar{B}}, g_{\bar{B}}, \bar{B}\right)$ is a classical solution of the optimality system

$$
\begin{cases}\partial_{t} f+v \cdot \partial_{x} f-\partial_{x} \psi_{f} \cdot \partial_{v} f+(v \times B) \cdot \partial_{v} f=0, & \left.f\right|_{t=0}=\AA \\ \partial_{t} g+v \cdot \partial_{x} g-\partial_{x} \psi_{f} \cdot \partial_{v} g+(v \times B) \cdot \partial_{v} g=\Phi_{f, g} \chi, & \left.g\right|_{t=T}=f(T)-f_{d} \\ B(t, x)=-\frac{1}{4 \pi \lambda} \iint \frac{1}{|x-y|} w \times \partial_{v} f(t, y, w) g(t, y, w) \mathrm{d}(y, w) .\end{cases}
$$

For all $t \in[0, T]$, supp $f_{\bar{B}}(t) \subset B_{R}(0)$ and $\operatorname{supp} g_{\bar{B}}(t) \subset B_{R^{*}}(0)$.

Proof From Theorem 32 we know that $\bar{B} \in C\left([0, T] ; C_{b}^{2}\right)$. Thus by Theorem 13 the solution $f_{\bar{B}}$ is classical, i.e., $f_{\bar{B}} \in C^{1}\left([0, T] \times \mathbb{R}^{6}\right)$ with supp $f_{\bar{B}}(t) \subset B_{R}(0)$ for all $t \in[0, T]$. We can use the decomposition $g_{\bar{B}}=f_{\bar{B}}+h_{\bar{B}}$ from the proof of Theorem 31 and from Proposition 24 we can easily deduce that $g_{\bar{B}}$ is classical, i.e., $g_{\bar{B}} \in C^{1}\left([0, T] \times \mathbb{R}^{6}\right)$ with supp $g_{\bar{B}}(t) \subset B_{R^{*}}(0)$ for all $t \in[0, T]$. The rest is obvious due to the construction of $f_{\bar{B}}, g_{\bar{B}}$ and Theorem 32 .

\subsubsection{A sufficient condition for local optimality}

To motivate the following approach let us at first consider the following example: Suppose that $\varphi: \mathbb{R}^{d} \rightarrow \mathbb{R}$ is a twice continuously differentiable function and let $U$ be a convex open subset of $\mathbb{R}^{d}$. Now, if there exists some point $x \in \bar{U}$ such that $\nabla \varphi(x) \cdot h \geq 0$ for all $h \in \mathbb{R}^{d}$ with $x+h \in \bar{U}$ and $D_{x}^{2} \varphi(x)$ is strictly positive definit, we can conclude that $x$ is a strict local minimum of $\varphi$. Again, this fact can be generalized to functionals on Banach spaces using the Fréchet derivatives of first and second order.

To prove that our cost-functional is twice continuously Fréchet differentiable we will need Fréchet differentiability of first order of the costate.

Lemma 34 Let $g .: \mathbb{B}_{K} \rightarrow C\left([0, T] ; L^{2}\left(\mathbb{R}^{6}\right)\right), B \mapsto g_{B}$ denote the field-costate operator. For any field $B \in \mathbb{B}_{K}$ and any direction $H \in \mathcal{V}$ there exists a unique strong solution $g_{B}^{H} \in H^{1}(] 0, T\left[\times \mathbb{R}^{6}\right)$ of the final value problem

$$
\left\{\begin{array}{l}
\partial_{t} g+v \cdot \partial_{x} g-\partial_{x} \psi_{f_{B}^{\prime}[H]} \cdot \partial_{v} g_{B}-\partial_{x} \psi_{f_{B}} \cdot \partial_{v} g+(v \times B) \cdot \partial_{v} g+(v \times H) \cdot \partial_{v} g_{B} \\
\quad=\Phi_{f_{B}, g} \chi-\Phi_{g_{B}, f_{B}^{\prime}[H]} \chi \\
\left.g\right|_{t=T}=0 .
\end{array}\right.
$$


Then the following holds:

(a) Let $t \in[0, T]$ be arbitrary. Then $g .(t)$ is Fréchet differentiable on $\stackrel{\circ}{\mathbb{B}}_{K}$ with respect to the $L^{2}\left(\mathbb{R}^{6}\right)$-norm, i.e., for any $B \in \stackrel{\circ}{B}_{K}$ there exists a unique linear operator $g_{B}^{\prime}(t): \mathcal{V} \rightarrow L^{2}\left(\mathbb{R}^{6}\right)$ such that

$$
\begin{gathered}
\forall \varepsilon>0 \exists \delta>0 \forall H \in \mathcal{V} \text { with }\|H\|_{\mathcal{V}}<\delta: \\
B+H \in \stackrel{\circ}{B}_{K} \quad \text { and } \quad \frac{\left\|g_{B+H}(t)-g_{B}(t)-g_{B}^{\prime}(t)[H]\right\|_{L^{2}}}{\|H\|_{L^{2}\left(0, T ; W^{2, \beta}\right.}}<\varepsilon .
\end{gathered}
$$

The Fréchet derivative is given by

$$
g_{B}^{\prime}(t)[H]=g_{B}^{H}(t), \quad H \in \mathcal{V} .
$$

(b) The control-costate operator $g$. is Fréchet differentiable on $\mathbb{B}_{K}$ with respect to the $C\left([0, T] ; L^{2}\left(\mathbb{R}^{6}\right)\right)$-norm, i.e., for any $B \in \mathbb{B}_{K}$ there exists a unique linear operator $g_{B}^{\prime}: \mathcal{V} \rightarrow C\left([0, T] ; L^{2}\left(\mathbb{R}^{6}\right)\right)$ such that

$$
\begin{gathered}
\forall \varepsilon>0 \exists \delta>0 \forall H \in \mathcal{V} \text { with }\|H\|_{\mathcal{V}}<\delta: \\
B+H \in \stackrel{\circ}{B}_{K} \quad \text { and } \quad \frac{\left\|g_{B+H}-g_{B}-g_{B}^{\prime}[H]\right\|_{C\left([0, T] ; L^{2}\right)}}{\|H\|_{L^{2}\left(0, T ; W^{2, \beta}\right)}}<\varepsilon .
\end{gathered}
$$

The Fréchet derivative is given by

$$
g_{B}^{\prime}[H]=g_{B}^{H}, \quad H \in \mathcal{V} .
$$

(c) For all $B, H \in \stackrel{\circ}{B}_{K}$, the solution $g_{B}^{H}$ depends Hölder-continuously on $B$ in such a way that there exists some constant $C>0$ depending only on $\stackrel{\circ}{f}, T, K$ and $\beta$ such that

$$
\sup _{\|H\|_{\mathcal{V}} \leq 1}\left\|g_{A}^{\prime}[H]-g_{B}^{\prime}[H]\right\|_{L^{2}\left(0, T ; L^{2}\right)} \leq C\|A-B\|_{L^{2}\left(0, T ; W^{2, \beta}\right)}^{\gamma}, A, B \in \stackrel{\circ}{\mathbb{B}}_{K}
$$

Comment As $K$ was arbitrary the above results hold true if $\mathbb{B}_{K}$ is replaced by $\stackrel{\circ}{B}_{2 K}$. Hence they are especially true for $B \in \mathbb{B}_{K}$.

Proof First note that the system (6.11) is of the type (5.1) where the quantities correspond in the following way:

\begin{tabular}{cccl}
\multicolumn{2}{l}{ System $(5.1)$} & System $(6.11)$ & \\
\hline$r_{0}$ & $\hat{=}$ & $R^{*}$ & $\geq 0$ \\
$r_{1}$ & $\hat{=}$ & 0 & $\geq 0$ \\
$r_{2}$ & $\hat{=}$ & $R^{*}$ & $>r_{1}$ \\
$\mathbf{a}$ & $\hat{=}$ & $f_{B}$ & $\in C\left([0, T] ; C_{b}^{1}\right)$ \\
$\mathbf{b}$ & $\hat{=}$ & $-(v \times H) \cdot \partial_{v} g_{B}+\partial_{x} \psi_{f_{B}^{\prime}[H]} \cdot \partial_{v} g_{B}-\Phi_{g_{B}, f_{B}^{\prime}[H]} \chi$ & $\in L^{2}\left(0, T ; C_{b} \cap H^{1}\right)$ \\
$\mathbf{f}$ & $\hat{=}$ & 0 & $\in C_{c}^{2}\left(\mathbb{R}^{6}\right)$
\end{tabular}



$\begin{array}{ll}\text { A } & \hat{=} \\ \mathrm{B} & \hat{=} \\ \mathrm{C} & \hat{=} \\ \chi & \hat{=}\end{array}$
$-\partial_{x} \psi_{f_{B}}$
$B$
$\in C\left([0, T] ; C^{1, \gamma}\right)$
$\in L^{2}\left(0, T ; C^{1, \gamma}\right)$
$\in C\left([0, T] ; C_{b}^{1}\right)$
$\in C_{c}^{1}\left(\mathbb{R}^{6}\right)$

This means that the coefficients of (6.11) satisfy the regularity conditions (5.10) of Corollary 26 (a). Hence (6.11) has a strong solution $g_{B}^{H} \in L^{\infty} \cap H^{1}(] 0, T\left[\times \mathbb{R}^{6}\right)$.

To prove Fréchet differentiability of the field-costate operator we must consider the difference $g_{B+H}-g_{B}$ with $B \in \stackrel{\circ}{B}_{K}$ and $H \in \mathcal{V}$ such that $B+H \in \stackrel{\circ}{\mathbb{B}}_{K}$. Therefore we will assume that $\|H\|_{L^{2}\left(0, T, W^{2, \beta}\right)}<\delta$ for some sufficiently small $\delta>0$. Again, we will expand the nonlinear terms in the Vlasov equation (6.6) to pick out the linear parts. Recall the decomposition $f_{B+H}-f_{B}=f_{B}^{\prime}[H]+f_{\mathcal{R}}$ from the proof of Theorem 27. Then

$$
\begin{aligned}
\partial_{x} \psi_{f_{B+H}} \cdot \partial_{v} g_{B+H}-\partial_{x} \psi_{f_{B}} \cdot \partial_{v} g_{B} \\
=\partial_{x} \psi_{f_{B}} \cdot \partial_{v}\left(g_{B+H}-g_{B}\right)+\partial_{x} \psi_{\left(f_{B+H}-f_{B}\right)} \cdot \partial_{v} g_{B}+\partial_{x} \psi_{\left(f_{B+H}-f_{B}\right)} \cdot \partial_{v}\left(g_{B+H}-g_{B}\right), \\
=\partial_{x} \psi_{f_{B}} \cdot \partial_{v}\left(g_{B+H}-g_{B}\right)+\partial_{x} \psi_{f_{B}^{\prime}[H]} \cdot \partial_{v} g_{B} \\
\quad+\partial_{x} \psi_{f_{R}} \cdot \partial_{v} g_{B}+\partial_{x} \psi_{\left(f_{B+H}-f_{B}\right)} \cdot \partial_{v}\left(g_{B+H}-g_{B}\right) \\
=\partial_{x} \psi_{f_{B}} \cdot \partial_{v}\left(g_{B+H}-g_{B}\right)+\partial_{x} \psi_{f_{B}^{\prime}[H]} \cdot \partial_{v} g_{B}+\mathcal{R}_{1}, \\
(v \times(B+H)) \cdot \partial_{v} g_{B+H}-(v \times B) \cdot \partial_{v} g_{B} \\
=(v \times B) \cdot \partial_{v}\left(g_{B+H}-g_{B}\right)+(v \times H) \cdot \partial_{v} g_{B}+(v \times H) \cdot \partial_{v}\left(g_{B+H}-g_{B}\right) \\
=(v \times B) \cdot \partial_{v}\left(g_{B+H}-g_{B}\right)+(v \times H) \cdot \partial_{v} g_{B}+\mathcal{R}_{2}, \\
\Phi_{f_{B+H}}, g_{B+H} \chi-\Phi_{f_{B}, g_{B}} \chi \\
=\Phi_{f_{B+H}-f_{B}, g_{B}} \chi+\Phi_{f_{B}, g_{B+H}-g_{B}} \chi+\Phi_{f_{B+H}-f_{B}, g_{B+H}-g_{B}} \chi \\
=\Phi_{f_{B}^{\prime}[H], g_{B}} \chi+\Phi_{f_{B}, g_{B+H}-g_{B}} \chi+\Phi_{f_{\mathcal{R}}, g_{B}} \chi+\Phi_{f_{B+H}-f_{B}, g_{B+H}-g_{B}} \chi \\
=-\Phi_{g_{B}, f_{B}^{\prime}[H]} \chi+\Phi_{f_{B}, g_{B+H}-g_{B}} \chi+\mathcal{R}_{3}
\end{aligned}
$$

where

$$
\begin{gathered}
\mathcal{R}_{1}:=\partial_{x} \psi_{f_{\mathcal{R}}} \cdot \partial_{v} g_{B}+\partial_{x} \psi_{\left(f_{B+H}-f_{B}\right)} \cdot \partial_{v}\left(g_{B+H}-g_{B}\right) \\
\mathcal{R}_{2}:=(v \times H) \cdot \partial_{v}\left(g_{B+H}-g_{B}\right), \quad \mathcal{R}_{3}:=\Phi_{f_{\mathcal{R}}, g_{B}} \chi+\Phi_{f_{B+H}-f_{B}, g_{B+H}-g_{B}} \chi
\end{gathered}
$$

are nonlinear remainders. We already know that

$$
\begin{gathered}
\left\|f_{\mathcal{R}}\right\|_{L^{2}} \leq C\|H\|_{L^{2}\left(0, T ; W^{2, \beta}\right)}^{1+\gamma}, \\
\left\|f_{B+H}-f_{B}\right\|_{C\left([0, T] ; C_{b}\right)} \leq C\|H\|_{L^{2}\left(0, T ; W^{2, \beta}\right)}, \\
\left\|g_{B+H}-g_{B}\right\|_{C\left([0, T] ; C_{b}\right)} \leq C\|H\|_{L^{2}\left(0, T ; W^{2, \beta}\right)}, \\
\left\|f_{B+H}-f_{B}\right\|_{C\left([0, T] ; C_{b}^{1}\right)} \leq C\|H\|_{L^{2}\left(0, T ; W^{2, \beta}\right)}^{\gamma}, \\
\left\|g_{B+H}-g_{B}\right\|_{C\left([0, T] ; C_{b}^{1}\right)} \leq C\|H\|_{L^{2}\left(0, T ; W^{2, \beta}\right)}^{\gamma}
\end{gathered}
$$

and hence, using Proposition 8 and (5.5), the term $\mathcal{R}:=\mathcal{R}_{1}-\mathcal{R}_{2}+\mathcal{R}_{3}$ can be bounded by $\|\mathcal{R}\|_{L^{2}\left(0, T ; L^{2}\right)} \leq C\|H\|_{L^{2}\left(0, T ; W^{2, \beta}\right)}^{1+\gamma}$. 
Obviously, $g_{B+H}-g_{B}$ is a strong solution of the initial value problem

$$
\left\{\begin{array}{l}
\begin{array}{rl}
\partial_{t} g & +v \cdot \partial_{x} g-\partial_{x} \psi_{f_{B}^{\prime}[H]} \cdot \partial_{v} g_{B}-\partial_{x} \psi_{f_{B}} \cdot \partial_{v} g+(v \times B) \cdot \partial_{v} g+(v \times H) \cdot \partial_{v} g_{B} \\
\quad & =\Phi_{f_{B}, g} \chi-\Phi_{g_{B}, f_{B}^{\prime}[H]} \chi+\mathcal{R}
\end{array} \\
\left.g\right|_{t=T}=0 .
\end{array}\right.
$$

As the coefficients of this system satisfy the conditions (5.10) of Corollary 26 (a), we can conclude that this solution is unique. Corollary 26 (a) also implies that the system

$$
\left\{\begin{array}{l}
\partial_{t} g+v \cdot \partial_{x} g-\partial_{x} \psi_{f_{B}} \cdot \partial_{v} g+(v \times B) \cdot \partial_{v} g=\Phi_{f_{B}, g} \chi+\mathcal{R} \\
\left.g\right|_{t=T}=0
\end{array}\right.
$$

has a unique strong solution that will be denoted by $g_{\mathcal{R}}$. Then $g_{B}^{H}+g_{\mathcal{R}}$ is a solution of (6.12) and hence $g_{B+H}-g_{B}=g_{B}^{H}+g_{\mathcal{R}}$ because of uniqueness. Moreover

$$
\begin{aligned}
\left\|g_{\mathcal{R}}(t)\right\|_{L^{2}}^{2} & =2 \int_{t}^{T} g_{\mathcal{R}}(s)\left(\Phi_{f_{B}, g_{\mathcal{R}}}(s) \chi+\mathcal{R}(s)\right) \mathrm{d} s \\
& \leq C \int_{t}^{T}\left\|g_{\mathcal{R}}(s)\right\|_{L^{2}}^{2}+\left\|g_{\mathcal{R}}(s)\right\|_{L^{2}}\|\mathcal{R}(s)\|_{L^{2}} \mathrm{~d} s
\end{aligned}
$$

and thus applying at first the standard version and then the quadratic version of Gronwall's lemma yields

$$
\left\|g_{\mathcal{R}}(t)\right\|_{L^{2}} \leq C \int_{t}^{T}\|\mathcal{R}(s)\|_{L^{2}} \mathrm{~d} s \leq C\|H\|_{L^{2}\left(0, T ; W^{2, \beta}\right)}^{1+\gamma} .
$$

Let now $\varepsilon>0$ be arbitrary. Then for all $t \in[0, T]$,

$$
\frac{\left\|g_{B+H}(t)-g_{B}(t)-g_{B}^{H}(t)\right\|_{L^{2}}}{\|H\|_{L^{2}\left(0, T ; W^{2, \beta}\right)}}=\frac{\left\|g_{\mathcal{R}}(t)\right\|_{L^{2}}}{\|H\|_{L^{2}\left(0, T ; W^{2, \beta}\right)}} \leq C\|H\|_{L^{2}\left(0, T ; W^{2, \beta}\right)}^{\gamma}<\varepsilon
$$

if $\delta$ is sufficiently small. Since the inequality holds for all $t \in[0, T]$ and all the terms depend continuously on time this also means that

$$
\frac{\left\|g_{B+H}-g_{B}-g_{B}^{H}\right\|_{C\left([0, T] ; L^{2}\right)}}{\|H\|_{L^{2}\left(0, T ; W^{2, \beta}\right)}}=\max _{t \in[0, T]} \frac{\left\|g_{B+H}(t)-g_{B}(t)-g_{B}^{H}(t)\right\|_{L^{2}}}{\|H\|_{L^{2}\left(0, T ; W^{2, \beta}\right)}}<\varepsilon
$$

Hence the assertions (a) and (b) are proved and the Fréchet derivative is determined by the system (6.11).

The proof of (c) is very similar to the proof of Theorem 27 as we already know that $f_{B}$, $g_{B}$ and $f_{B}^{\prime}[H]$ are Hölder continuous with respect to $B$. 
Continuous differentiability of the cost functional then follows:

Corollary 35 The cost functional $J$ of the optimization problem (6.2) is twice Fréchet differentiable on $\stackrel{\circ}{B}_{K}$. The Fréchet derivative of second order at the point $B \in \stackrel{\circ}{B}_{K}$ can be described as a bilinear operator $J^{\prime \prime}(B): \mathcal{V}^{2} \rightarrow \mathbb{R}$ that is given by

$$
\begin{aligned}
J^{\prime \prime}(B)\left[H_{1}, H_{2}\right]= & \lambda\left\langle D_{x} H_{1}, D_{x} H_{2}\right\rangle_{L^{2}\left([0, T] \times \mathbb{R}^{3}\right)} \\
& -\int_{[0, T] \times \mathbb{R}^{6}}\left(v \times H_{1}\right) \cdot\left(\partial_{v} f_{B} g_{B}^{\prime}\left[H_{2}\right]-\partial_{v} g_{B} f_{B}^{\prime}\left[H_{2}\right]\right) \mathrm{d}(t, x, v)
\end{aligned}
$$

for all $H_{1}, H_{2} \in \mathcal{V}$. Moreover there exists some constant $C>0$ depending only on $\stackrel{\circ}{f}$, $f_{d}, T, K$ and $\beta$ such that for all $B, \tilde{B} \in \stackrel{\circ}{B}_{K}$,

$$
\left\|J^{\prime \prime}(B)-J^{\prime \prime}(\tilde{B})\right\| \leq C\|B-\tilde{B}\|_{L^{2}\left(0, T ; W^{2, \beta}\right)}^{\gamma}
$$

where

$$
\left\|J^{\prime \prime}(B)\right\|=\sup \left\{\left|J^{\prime \prime}(B)\left[H_{1}, H_{2}\right]\right| \mid\left\|H_{1}\right\|_{L^{2}\left(0, T ; W^{2, \beta}\right)}=1,\left\|H_{2}\right\|_{L^{2}\left(0, T ; W^{2, \beta}\right)}=1\right\}
$$

denotes the operator norm. This means that $J$ is twice continuously differentiable.

Comment By definition the Fréchet derivative of second order is the Fréchet derivative of the Fréchet derivative of first order. This means that, in the proper sense, it is an operator $J^{\prime \prime}(B): \mathcal{V} \rightarrow \mathcal{L}(\mathcal{V} ; \mathcal{L}(\mathcal{V} ; \mathbb{R}))$. Because of the two linear dependences we can equivalently consider the Fréchet derivative of second order as a bilinear operator $J^{\prime \prime}(B): \mathcal{V}^{2} \rightarrow \mathbb{R}$ as it was done in the above proposition. Since $K$ was arbitrary, $\mathbb{B}_{K}$ can be replaced by $\mathbb{B}_{2 K}$ and hence all results of this proposition are also true on $\mathbb{B}_{K}$ instead of $\stackrel{\circ}{\mathbb{B}}_{K}$.

Proof Theorem 27 and Theorem 34 provide the decompositions

$$
f_{B+H}-f_{B}=f_{B}^{\prime}[H]+f_{R}[H], \quad g_{B+H}-g_{B}=g_{B}^{\prime}[H]+g_{R}[H]
$$

for $B \in \mathbb{B}_{K}, H \in \mathcal{V}$ with $B+H \in \mathbb{B}_{K}$ where

$$
\left\|f_{R}[H]\right\|_{C\left([0, T] ; L^{2}\right)}=\mathrm{o}\left(\|H\|_{L^{2}\left(0, T ; W^{2, \beta}\right)}\right) \quad \text { and } \quad\left\|g_{R}[H]\right\|_{C\left([0, T] ; L^{2}\right)}=\mathrm{o}\left(\|H\|_{L^{2}\left(0, T ; W^{2, \beta}\right)}\right)
$$

if $\|H\|_{L^{2}\left(0, T ; W^{2, \beta}\right)}$ tends to 0 . We already know from Theorem 32 (a) that

$$
J^{\prime}(B)[H]=\lambda\left\langle D_{x} H, D_{x} B\right\rangle_{L^{2}}-\int_{[0, T] \times \mathbb{R}^{6}}(v \times H) \cdot \partial_{v} f_{B} g_{B} \mathrm{~d}(t, x, v)
$$

for all $B \in \mathbb{B}_{K}, H \in \mathcal{V}$. Let now $B \in \mathbb{B}_{K}$ and $H_{1}, H_{2} \in \mathcal{V}$ with $B+H_{2} \in \mathbb{B}_{K}$ be arbitrary . 
Then it holds that

$$
\begin{aligned}
J^{\prime}(B & \left.+H_{2}\right)\left[H_{1}\right]-J^{\prime}(B)\left[H_{1}\right] \\
= & \lambda\left\langle D_{x} H_{1}, D_{x} H_{2}\right\rangle_{L^{2}}-\int_{[0, T] \times \mathbb{R}^{6}}\left(v \times H_{1}\right) \cdot\left(\partial_{v} f_{B+H_{2}} g_{B+H_{2}}-\partial_{v} f_{B} g_{B}\right) \mathrm{d}(t, x, v) \\
= & \lambda\left\langle D_{x} H_{1}, D_{x} H_{2}\right\rangle_{L^{2}} \\
& -\int_{[0, T] \times \mathbb{R}^{6}}\left(v \times H_{1}\right) \cdot\left(\partial_{v} f_{B}\left(g_{B+H_{2}}-g_{B}\right)-\partial_{v} g_{B}\left(f_{B+H_{2}}-f_{B}\right)\right) \mathrm{d}(t, x, v) \\
& -\int_{[0, T] \times \mathbb{R}^{6}}\left(v \times H_{1}\right) \cdot\left(\partial_{v} f_{B+H_{2}}-\partial_{v} f_{B}\right)\left(g_{B+H_{2}}-g_{B}\right) \mathrm{d}(t, x, v) \\
= & \lambda\left\langle D_{x} H_{1}, D_{x} H_{2}\right\rangle_{L^{2}}-\int_{[0, T] \times \mathbb{R}^{6}}\left(v \times H_{1}\right) \cdot\left(\partial_{v} f_{B} g_{B}^{\prime}\left[H_{2}\right]-\partial_{v} g_{B} f_{B}^{\prime}\left[H_{2}\right]\right) \mathrm{d}(t, x, v)+\mathcal{R}
\end{aligned}
$$

where

$$
\begin{aligned}
\mathcal{R}:= & -\int_{[0, T] \times \mathbb{R}^{6}}\left(v \times H_{1}\right) \cdot\left(\partial_{v} f_{B} g_{R}\left[H_{2}\right]-\partial_{v} g_{B} f_{R}\left[H_{2}\right]\right) \mathrm{d}(t, x, v) \\
& -\int_{[0, T] \times \mathbb{R}^{6}}\left(v \times H_{1}\right) \cdot\left(\partial_{v} f_{B+H_{2}}-\partial_{v} f_{B}\right)\left(g_{B+H_{2}}-g_{B}\right) \mathrm{d}(t, x, v) .
\end{aligned}
$$

Now

$$
\begin{aligned}
|\mathcal{R}| \leq & R \sqrt{T}\left\|H_{1}\right\|_{L^{2}\left(0, T ; L^{2}\right)}\left(\left\|\partial_{v} f_{B}\right\|_{\infty}\left\|g_{R}\left[H_{2}\right]\right\|_{C\left([0, T] ; L^{2}\right)}+\left\|\partial_{v} g_{B}\right\|_{\infty}\left\|f_{R}\left[H_{2}\right]\right\|_{C\left([0, T] ; L^{2}\right)}\right) \\
& +R\left\|H_{1}\right\|_{L^{2}\left(0, T ; L^{2}\right)}\left(\left\|\partial_{v} f_{B+H_{2}}-\partial_{v} f_{B}\right\|_{\infty}\left\|g_{B+H_{2}}-g_{B}\right\|_{L^{2}\left(0, T ; L^{2}\right)}\right) \\
= & \left\|H_{1}\right\|_{L^{2}\left(0, T ; L^{2}\right)} o\left(\left\|H_{2}\right\|_{L^{2}\left(0, T ; W^{2, \beta}\right)}\right),
\end{aligned}
$$

and hence $J$ is twice Fréchet differentiable at the point $B$ and the Fréchet derivative is given by

$$
\begin{aligned}
J^{\prime \prime}(B)\left[H_{1}, H_{2}\right]= & \lambda\left\langle D_{x} H_{1}, D_{x} H_{2}\right\rangle_{L^{2}\left([0, T] \times \mathbb{R}^{3}\right)} \\
& -\int_{[0, T] \times \mathbb{R}^{6}}\left(v \times H_{1}\right) \cdot\left(\partial_{v} f_{B} g_{B}^{\prime}\left[H_{2}\right]-\partial_{v} g_{B} f_{B}^{\prime}\left[H_{2}\right]\right) \mathrm{d}(t, x, v) .
\end{aligned}
$$

This means that $J$ is twice Fréchet differentiable and its Fréchet derivative of second order at the point $B$ with directions $H_{1}$ and $H_{2}$ is given by the above expression.

To prove continuity let $B, \tilde{B} \in \mathbb{B}_{K}$ and $H_{1}, H_{2} \in \mathcal{V}$ be arbitrary and suppose that $\left\|H_{i}\right\|_{\mathcal{V}} \leq 1$ for $i=1,2$. Then

$$
\begin{aligned}
& \left|J^{\prime \prime}(B)\left[H_{1}, H_{2}\right]-J^{\prime \prime}(\tilde{B})\left[H_{1}, H_{2}\right]\right| \\
& \quad=\left|\int_{0}^{T} \int\left(v \times H_{1}\right) \cdot\left(\partial_{v} f_{B} g_{B}^{\prime}\left[H_{2}\right]-\partial_{v} f_{\tilde{B}} g_{\tilde{B}}^{\prime}\left[H_{2}\right]-\partial_{v} g_{B} f_{B}^{\prime}\left[H_{2}\right]+\partial_{v} g_{\tilde{B}} f_{\tilde{B}}^{\prime}\left[H_{2}\right]\right) \mathrm{d} z \mathrm{~d} t\right|
\end{aligned}
$$




$$
\begin{gathered}
\leq R \int_{0}^{T}\left\|H_{1}(t)\right\|_{\infty}\left[\left\|\partial_{v} f_{B}(t)-\partial_{v} f_{\tilde{B}}(t)\right\|_{L^{2}}\left\|g_{\tilde{B}}^{\prime}\left[H_{2}\right](t)\right\|_{L^{2}}\right. \\
+\left\|\partial_{v} f_{B}(t)\right\|_{L^{2}}\left\|g_{B}^{\prime}\left[H_{2}\right](t)-g_{\tilde{B}}^{\prime}\left[H_{2}\right](t)\right\|_{L^{2}} \\
+\left\|\partial_{v} g_{B}(t)-\partial_{v} g_{\tilde{B}}(t)\right\|_{L^{2}}\left\|f_{B}^{\prime}\left[H_{2}\right](t)\right\|_{L^{2}} \\
\left.+\left\|\partial_{v} g_{\tilde{B}}(t)\right\|_{L^{2}}\left\|f_{B}^{\prime}\left[H_{2}\right](t)-f_{\tilde{B}}^{\prime}\left[H_{2}\right](t)\right\|_{L^{2}}\right] \mathrm{d} t \\
\leq R K\left[\left\|g_{\tilde{B}}^{\prime}\left[H_{2}\right]\right\|_{L^{2}\left(0, T ; L^{2}\right)}+\left\|\partial_{v} f_{B}\right\|_{C\left(0, T ; L^{2}\right)}+\left\|f_{B}^{\prime}\left[H_{2}\right]\right\|_{L^{2}\left(0, T ; L^{2}\right)}\right. \\
\left.+\left\|\partial_{v} g_{\tilde{B}}(t)\right\|_{C\left(0, T ; L^{2}\right)}\right]\|B-\tilde{B}\|_{L^{2}\left(0, T ; W^{2, \beta}\right)}^{\gamma} \\
\leq C\|B-\tilde{B}\|_{L^{2}\left(0, T ; W^{2, \beta}\right)}^{\gamma}
\end{gathered}
$$

where the constant $C>0$ depends only on $\stackrel{\circ}{f}, f_{d}, T, K$ and $\beta$. This directly yields continuity of the second order derivative with respect to the operator norm.

The following theorem provides a sufficient condition for local optimality:

Theorem 36 Suppose that $\bar{B} \in \mathbb{B}_{K}$ and let $f_{\bar{B}}$ and $g_{\bar{B}}$ be its induced state and costate. Let $0<\alpha<2+\gamma$ be any real number. We assume that the variation inequality

$$
\int_{[0, T] \times \mathbb{R}^{3}}\left(-\lambda \Delta_{x} \bar{B}+\int_{\mathbb{R}^{3}} v \times \partial_{v} f_{\bar{B}} g_{\bar{B}} \mathrm{~d} v\right) \cdot(B-\bar{B}) \mathrm{d}(t, x)=J^{\prime}(\bar{B})[B-\bar{B}] \geq 0
$$

holds for all $B \in \mathbb{B}_{K}$ and that there exists some constant $\varepsilon>0$ such that

$$
\begin{aligned}
& \lambda\left\|D_{x} H\right\|_{L^{2}\left([0, T] \times \mathbb{R}^{3}\right)}^{2}-\int_{[0, T] \times \mathbb{R}^{6}}(v \times H) \cdot\left(\partial_{v} f_{\bar{B}} g_{\bar{B}}^{\prime}[H]-\partial_{v} g_{\bar{B}} f_{\bar{B}}^{\prime}[H]\right) \mathrm{d}(t, x, v) \\
& =J^{\prime \prime}(\bar{B})[H, H] \geq \varepsilon\|H\|_{L^{2}\left([0, T] \times \mathbb{R}^{3}\right)}^{\alpha}
\end{aligned}
$$

holds for all $H \in \mathcal{V}$.

Then $J$ satisfies the following growth condition: There exists $\delta>0$ such that for all $B \in \mathbb{B}_{K}$ with $\|B-\bar{B}\|_{L^{2}\left(0, T ; W^{2, \beta}\right)}<\delta$,

$$
J(B) \geq J(\bar{B})+\frac{\varepsilon}{4}\|B-\bar{B}\|_{L^{2}\left(0, T ; W^{2, \beta}\right)}^{\alpha}
$$

and hence $\bar{B}$ is a strict local minimizer of $J$ on the set $\mathbb{B}_{K}$.

Proof Let $B \in \mathbb{B}_{K}$ be arbitrary. We define the auxillary function $F:[0,1] \rightarrow \mathbb{R}_{0}^{+}$, $s \mapsto J(\bar{B}+s(B-\bar{B}))$. Then $F$ is twice continuously differentiable by chain rule and Taylor expansion yields

$$
F(1)=F(0)+F^{\prime}(0)+\frac{1}{2} F^{\prime \prime}(\vartheta)
$$


for some $\vartheta \in] 0,1[$. This yields

$$
\begin{aligned}
J(B)= & J(\bar{B})+J^{\prime}(\bar{B})[B-\bar{B}]+\frac{1}{2} J^{\prime \prime}(\bar{B}+\vartheta(B-\bar{B}))[B-\bar{B}, B-\bar{B}] \\
\geq & J(\bar{B})+\frac{1}{2} J^{\prime \prime}(\bar{B}+\vartheta(B-\bar{B}))[B-\bar{B}, B-\bar{B}] \\
= & J(\bar{B})+\frac{1}{2} J^{\prime \prime}(\bar{B})[B-\bar{B}, B-\bar{B}] \\
& +\frac{1}{2}\left(J^{\prime \prime}(\bar{B}+\vartheta(B-\bar{B}))-J^{\prime \prime}(\bar{B})\right)[B-\bar{B}, B-\bar{B}]
\end{aligned}
$$

Now, according to Corollary 35,

$$
\begin{aligned}
& \left|\left(J^{\prime \prime}(\bar{B}+\vartheta(B-\bar{B}))-J^{\prime \prime}(\bar{B})\right)[B-\bar{B}, B-\bar{B}]\right| \\
& \quad \leq\left\|J^{\prime \prime}(\bar{B}+\vartheta(B-\bar{B}))-J^{\prime \prime}(\bar{B})\right\|\|B-\bar{B}\|_{L^{2}\left(0, T ; W^{2, \beta}\right)}^{2} \\
& \quad \leq C\|B-\bar{B}\|_{L^{2}\left(0, T ; W^{2, \beta}\right)}^{2+\gamma}
\end{aligned}
$$

Suppose now that $\|B-\bar{B}\|_{L^{2}\left(0, T ; W^{2, \beta}\right)}<\delta$ for some $\delta>0$. Then

$$
\begin{aligned}
& \left|\left(J^{\prime \prime}(\bar{B}+\vartheta(B-\bar{B}))-J^{\prime \prime}(\bar{B})\right)[B-\bar{B}, B-\bar{B}]\right| \\
& \quad \leq C \delta^{2+\gamma-\alpha}\|B-\bar{B}\|_{L^{2}\left(0, T ; W^{2, \beta}\right)}^{\alpha} \\
& \quad \leq \frac{\varepsilon}{2}\|B-\bar{B}\|_{L^{2}\left(0, T ; W^{2, \beta}\right)}^{\alpha}
\end{aligned}
$$

if $\delta$ is sufficiently small. In this case

$$
J(B) \geq J(\bar{B})+\frac{\varepsilon}{4}\|B-\bar{B}\|_{L^{2}\left(0, T ; W^{2, \beta}\right)}^{\alpha} .
$$

This especially means that $J(B)>J(\bar{B})$ for all $B \in B_{\delta}(\bar{B}) \cap \mathbb{B}_{K}$ and consequently $\bar{B}$ is a strict local minimizer of $J$.

\subsubsection{Uniqueness of the optimal solution on small time intervals}

We know from Corollary 33 that for any locally optimal solution $\bar{B} \in \stackrel{\circ}{\mathbb{B}}_{K}$ the triple $\left(f_{\bar{B}}, g_{\bar{B}}, \bar{B}\right)$ is a classical solution of the optimality system

$$
\begin{cases}\partial_{t} f+v \cdot \partial_{x} f-\partial_{x} \psi_{f} \cdot \partial_{v} f+(v \times B) \cdot \partial_{v} f=0, & \left.f\right|_{t=0}=\AA \\ \partial_{t} g+v \cdot \partial_{x} g-\partial_{x} \psi_{f} \cdot \partial_{v} g+(v \times B) \cdot \partial_{v} g=\Phi_{f, g}, & \left.g\right|_{t=T}=f(T)-f_{d} \\ B(t, x)=-\frac{1}{4 \pi \lambda} \iint \frac{1}{|x-y|} w \times \partial_{v} f(t, y, w) g(t, y, w) \mathrm{d}(y, w)\end{cases}
$$

The following theorem states that the solution of this system of equations is unique if the final time $T$ is small compared to $\lambda$. As we will have to adjust $\frac{T}{\lambda}$ it is necessary to assume that $0<\lambda \leq \lambda_{0}$ for some constant $\lambda_{0}>0$. Of course large regularaization parameters $\lambda$ do not make sense in our model, so we will just assume that $\lambda_{0}=1$. 
Theorem 37 Suppose that $\lambda \in] 0,1]$ and let us assume that there exists a classical solution $(f, g, B)$ of the optimality system (6.18), i.e., $B \in C\left([0, T] ; C_{b}^{1}\left(\mathbb{R}^{3} ; \mathbb{R}^{3}\right)\right)$ and $f, g \in C^{1}\left([0, T] \times \mathbb{R}^{6}\right)$ with supp $f(t)$, supp $g(t) \subset B_{r}(0)$ for some radius $r>0$.

Then this solution is unique if the quotient $\frac{T}{\lambda}$ is sufficiently small.

Proof Suppose that the triple $(\tilde{f}, \tilde{g}, \tilde{B})$ is another classical solution that is satisfying the support condition with radius $\tilde{r}$. Without loss of generality we assume that $r=\tilde{r}$. Let $C=C(T) \geq 0$ denote some generic constant that may depend on $T, \stackrel{f}{f}, f_{d}, r$, $\|\chi\|_{C_{b}^{1}}$ and the $C\left([0, T] ; C_{b}^{1}\right)$-norm of $f, \tilde{f}, g$ and $\tilde{g}$. We can assume that $C=C(T)$ is monotonically increasing in $T$. First of all, by integration by parts,

$$
\|B(t)-\tilde{B}(t)\|_{\infty} \leq \frac{C}{\lambda}\|g(t)-\tilde{g}(t)\|_{\infty}+\frac{C}{\lambda}\|f(t)-\tilde{f}(t)\|_{\infty}, \quad t \in[0, T]
$$

Let now $Z$ and $\tilde{Z}$ denote the solutions of the characteristic system of the Vlasov equation to the fields $B$ and $\tilde{B}$ satisfying $Z(t, t, z)=z$ and $\tilde{Z}(t, t, z)=z$ for any $t \in[0, T]$ and $z \in \mathbb{R}^{6}$. Then for any $s, t \in[0, T]$ (where $s \leq t$ without loss of generality) and $z \in \mathbb{R}^{6}$,

$$
\begin{aligned}
& |Z(s, t, z)-\tilde{Z}(s, t, z)| \\
& \quad \leq \int_{s}^{t} C|Z(\tau, t, z)-\tilde{Z}(\tau, t, z)|+C\left\|\partial_{x} \psi_{f}(\tau)-\partial_{x} \psi_{\tilde{f}}(\tau)\right\|_{\infty}+C\|B(\tau)-\tilde{B}(\tau)\|_{\infty} \mathrm{d} \tau \\
& \quad \leq \int_{s}^{t} C|Z(\tau, t, z)-\tilde{Z}(\tau, t, z)|+\frac{C}{\lambda}\|f(\tau)-\tilde{f}(\tau)\|_{\infty}+\frac{C}{\lambda}\|g(\tau)-\tilde{g}(\tau)\|_{\infty} \mathrm{d} \tau
\end{aligned}
$$

and hence

$$
|Z(s, t, z)-\tilde{Z}(s, t, z)| \leq C \int_{s}^{t} \frac{1}{\lambda}\|f(\tau)-\tilde{f}(\tau)\|_{\infty}+\frac{1}{\lambda}\|g(\tau)-\tilde{g}(\tau)\|_{\infty} \mathrm{d} \tau
$$

by Gronwall's lemma. Consequently

$$
\begin{aligned}
\|f(t)-\tilde{f}(t)\|_{\infty} & \leq C\|Z(0, t, \cdot)-\tilde{Z}(0, t, \cdot)\|_{\infty} \\
& \leq C \int_{0}^{t} \frac{1}{\lambda}\|f(\tau)-\tilde{f}(\tau)\|_{\infty}+\frac{1}{\lambda}\|g(\tau)-\tilde{g}(\tau)\|_{\infty} \mathrm{d} \tau
\end{aligned}
$$

which yields

$$
\|f(t)-\tilde{f}(t)\|_{\infty} \leq C \frac{1}{\lambda} \exp \left(C \frac{T}{\lambda}\right) \int_{0}^{t}\|g(\tau)-\tilde{g}(\tau)\|_{\infty} \mathrm{d} \tau
$$

and thus

$$
\|f-\tilde{f}\|_{C\left([0, T] ; C_{b}\right)} \leq C \frac{T}{\lambda} \exp \left(C \frac{T}{\lambda}\right)\|g-\tilde{g}\|_{C\left([0, T] ; C_{b}\right)} .
$$


For $z \in B_{r}(0)$ and $t \in[0, T]$ the representation formula of Proposition 24 yields

$$
\begin{aligned}
|g(t, z)-\tilde{g}(t, z)| & \\
\leq & \left|\left(f(T)-f_{d}\right)(Z(T, t, z))-\left(\tilde{f}(T)-f_{d}\right)(\tilde{Z}(T, t, z))\right| \\
& +\int_{t}^{T}\left|\left[\Phi_{f, g} \chi\right](\tau, Z(\tau, t, z))-\left[\Phi_{\tilde{f}, \tilde{g}} \chi\right](\tau, \tilde{Z}(\tau, t, z))\right| \mathrm{d} \tau \\
\leq & C\|Z(T, t, \cdot)-\tilde{Z}(T, t, \cdot)\|_{\infty} \\
& +\int_{t}^{T}\left|\Phi_{f, g}(\tau, X(\tau, t, z))-\Phi_{\tilde{f}, \tilde{g}}(\tau, X(\tau, t, z))\right| \mathrm{d} \tau \\
& +\int_{t}^{T}\left|\left[\Phi_{\tilde{f}, \tilde{g}} \chi\right](\tau, Z(\tau, t, z))-\left[\Phi_{\tilde{f}, \tilde{g}} \chi\right](\tau, \tilde{Z}(\tau, t, z))\right| \mathrm{d} \tau \\
\leq & C\left\|^{T} Z(T, t, \cdot)-\tilde{Z}(T, t, \cdot)\right\|_{\infty}+\int_{t}^{T}\left\|\Phi_{f, g}(\tau)-\Phi_{\tilde{f}, \tilde{g}}(\tau)\right\|_{L^{\infty}\left(B_{r}(0)\right)} \mathrm{d} \tau \\
& +C \int_{t}^{T}\left\|\Phi_{\tilde{f}, \tilde{g}}(\tau)\right\|_{W^{1, \infty}}\|Z(\tau, t, \cdot)-\tilde{Z}(\tau, t, \cdot)\|_{\infty} \mathrm{d} \tau .
\end{aligned}
$$

We already know from inequality (6.20) that for $t \leq \tau \leq T$,

$$
\|Z(\tau, t, \cdot)-\tilde{Z}(\tau, t, \cdot)\|_{\infty} \leq C \int_{t}^{\tau} \frac{1}{\lambda}\|f(\sigma)-\tilde{f}(\sigma)\|_{\infty}+\frac{1}{\lambda}\|g(\sigma)-\tilde{g}(\sigma)\|_{\infty} \mathrm{d} \sigma .
$$

Also recall that

$$
\left\|\Phi_{f, g}(\tau)\right\|_{W^{1, \infty}} \leq\left\|\Phi_{f, g}(\tau)\right\|_{\infty}+\left\|\Phi_{f, g}^{\prime}(\tau)\right\|_{\infty} \leq C\|f\|_{C\left([0, T] ; C_{b}^{1}\right)}\|g\|_{C\left([0, T] ; C_{b}^{1}\right)} \leq C
$$

for every $\tau \in[0, T]$. Moreover by Proposition 8,

$$
\begin{aligned}
\left\|\Phi_{f, g}(\tau)-\Phi_{\tilde{f}, \tilde{g}}(\tau)\right\|_{L^{\infty}\left(B_{r}(0)\right)} & \leq C\left\|\partial_{z} \tilde{g}\right\|_{\infty}\|f(\tau)-\tilde{f}(\tau)\|_{\infty}+C\left\|\partial_{z} f\right\|_{\infty}\|g(\tau)-\tilde{g}(\tau)\|_{\infty} \\
& \leq C\|f(\tau)-\tilde{f}(\tau)\|_{\infty}+C\|g(\tau)-\tilde{g}(\tau)\|_{\infty}
\end{aligned}
$$

for all $\tau \in[0, T]$. This implies that for all $t \in[0, T]$,

$$
\|g(t)-\tilde{g}(t)\|_{\infty} \leq C \int_{t}^{T} \frac{1}{\lambda}\|g(\tau)-\tilde{g}(\tau)\|_{\infty}+\frac{1}{\lambda}\|f(\tau)-\tilde{f}(\tau)\|_{\infty} \mathrm{d} \tau
$$

and hence

$$
\|g-\tilde{g}\|_{C\left([0, T] ; C_{b}\right)} \leq C \frac{T}{\lambda} \exp \left(C \frac{T}{\lambda}\right)\|f-\tilde{f}\|_{C\left([0, T] ; C_{b}\right)}
$$

by Gronwall's lemma. Inserting (6.22) in (6.21) yields

$$
\|f-\tilde{f}\|_{C\left([0, T] ; C_{b}\right)} \leq C\left(\frac{T}{\lambda}\right)^{2} \exp \left(C \frac{T}{\lambda}\right)\|f-\tilde{f}\|_{C\left([0, T] ; C_{b}\right)} .
$$


If now $\frac{T}{\lambda}$ is sufficiently small we have $C\left(\frac{T}{\lambda}\right)^{2} \exp \left(C \frac{T}{\lambda}\right)<1$ and we can conclude that $f=\tilde{f}$ on $[0, T] \times \mathbb{R}^{6}$. Then obviously $g=\tilde{g}$ by $(6.22)$ and $B=\tilde{B}$ by (6.19) which means uniqueness of the solution $(f, g, B)$.

If $\bar{B} \in \stackrel{\circ}{B}_{K}$ is a locally optimal solution, the following uniqueness result holds:

Corollary 38 Suppose that $\lambda \in] 0,1]$ and let $\bar{B} \in \stackrel{\circ}{B}_{K}$ be a locally optimal solution of the optimization problem (6.2). Then the tripel $\left(f_{\bar{B}}, g_{\bar{B}}, \bar{B}\right)$ is a classical solution of the optimality system (6.18) according to Corollary 33.

If now $\lambda \in] 0,1]$ and $\frac{T}{\lambda}$ is sufficiently small then $\bar{B}$ is the only locally optimal solution of the optimization problem $(6.2)$ in $\stackrel{\circ}{B}_{K}$.

Suppose that there is a globally optimal solution $B \in \stackrel{\circ}{B}_{K}$. Then $B=\bar{B}$ is the unique globally optimal solution in $\mathbb{B}_{K}$. However it is still possible that there are other globally optimal solutions in $\partial \mathbb{B}_{K}$.

Proof If $\lambda \in] 0,1]$ and $\frac{T}{\lambda}$ is sufficiently small then Proposition 37 ensures that $\bar{B}$ is the only locally optimal solution. Recall that there exists at least one globally optimal solution according to Theorem 28. Let us assume that there is a globally optimal solution $B \in \stackrel{\circ}{B}_{K}$. As any globally optimal solution is also locally optimal it follows that there is only one globally optimal solution in $\stackrel{\circ}{B}_{K}$ and thus $B=\bar{B}$. 


\subsection{Optimal control by a finite number of field coils}

\subsubsection{The model}

In real applications, for example in fusion research, the external magnetic field is to be generated by a finite number $N$ of field coils. Each coil generates a magnetic field of a certain shape $m_{i}=m_{i}(x)$ and its intensity at time $t$ is determined by a multiplier $u_{i}(t)$. This means that the magnetic field of the $i$-th field coil is given by $B_{i}(t, x)=u_{i}(t) m_{i}(t)$ and the complete external magnetic field is given by

$$
B(u)(t, x)=\sum_{i=1}^{N} u_{i}(t) m_{i}(x) .
$$

We will suppose that $m_{i} \in W^{2, \beta} \cap H^{1}\left(\mathbb{R}^{3} ; \mathbb{R}^{3}\right)$ for every index $i \in\{1, \ldots, N\}$ and, since real magnetic fields are always source-free, we may also assume that $\operatorname{div} m_{i}=0$. The intensity function $u_{i}$ is directly proportional to the intensity of the current that flows through the $i$-th coil. Now the vector $u=\left(u_{1}, \ldots, u_{N}\right)^{T}$ will be the control in our model. Therefore we will assume $u$ to be a $L^{2}\left([0, T] ; \mathbb{R}^{N}\right)$-function in order to ensure that the field $B(u)$ has the desired regularity. All of this is specified in the following definition:

Definition 39 Let $N$ be a fixed positive integer and $M>0$ be a real number. For every $i \in\{1, \ldots, N\}$ let $m_{i}=\left(m_{i 1}, m_{i 2}, m_{i 3}\right)^{T}$ be a fixed vector-valued function in $W^{2, \beta} \cap H^{1}\left(\mathbb{R}^{3} ; \mathbb{R}^{3}\right) \subset C^{1, \gamma}\left(\mathbb{R}^{3} ; \mathbb{R}^{3}\right)$ with $\left\|m_{i}\right\|_{W^{2, \beta}} \leq M$ and $\operatorname{div} m_{i}=0$ on $\mathbb{R}^{3}$ for all $i \in\{1,2,3\}$. Moreover let $a=\left(a_{1}, \ldots, a_{N}\right)^{T}$ and $b=\left(b_{1}, \ldots, b_{N}\right)^{T}$ be fixed functions in $L^{2}\left([0, T] ; \mathbb{R}^{N}\right)$ with $a_{i} \leq 0 \leq b_{i}$ almost everywhere on $[0, T]$ for all $i \in\{1, \ldots, N\}$. We define

$$
\begin{aligned}
& \mathbb{U}_{i}:=\left\{u \in L^{2}([0, T]) \mid a_{i} \leq u \leq b_{i} \text { a.e. on }[0, T]\right\}, \\
& \mathbb{U}:=\mathbb{U}_{1} \times \ldots \times \mathbb{U}_{N} .
\end{aligned}
$$

The set $\mathbb{U}$ will be referred to as the set of admissible controls. Moreover we define the operator

$$
B(\cdot): L^{2}\left([0, T] ; \mathbb{R}^{N}\right) \rightarrow L^{2}\left(0, T ; W^{2, \beta}\left(\mathbb{R}^{3} ; \mathbb{R}^{3}\right)\right), u \mapsto B(u)
$$

where

$$
B(u)(t, x):=\sum_{i=1}^{N} u_{i}(t) m_{i}(x) .
$$

The operator $B(\cdot)$ is referred to as the control-field operator.

This definition does only make sense if the fields that are generated by the control-field operator are admissible in the sense of Definition 9, i.e., we must find some constant $K>0$ such that $B(u) \in \mathbb{B}_{K}$ for all $u \in \mathbb{U}$. In this case the state $f_{B(u)}$ is well-defined but we have to know how it depends on the control $u$. Therefore we introduce another lemma: 


\section{Lemma 40}

(a) For any $i \in\{1, \ldots, N\}$ the set $\mathbb{U}_{i}$ is a bounded, convex and closed subset of $L^{2}([0, T])$ and thus it is weakly compact. The same holds for $\mathbb{U}$ as a subset of $L^{2}\left([0, T] ; \mathbb{R}^{N}\right)$.

(b) The operator $B(\cdot)$ is linear and continuous and there exists some constant $K>0$ depending only on $N, a, b$ and $M$ such that $B(\mathbb{U}) \subset \stackrel{\circ}{B}_{K / 2} \subset \mathbb{B}_{K}$, i.e., the controlfield operator provides only admissible fields.

(c) The control-field operator $B(\cdot)$ is continuously Fréchet-differentiable on $\mathbb{U}$ and its Fréchet derivative at the point $u \in \mathbb{U}$ is given by

$$
B^{\prime}(u)[h]=B(h) \quad \text { for all } \quad h \in L^{2}\left([0, T] ; \mathbb{R}^{N}\right) .
$$

(d) The control-state operator $f_{B(\cdot)}=f . \circ B(\cdot)$ is Fréchet-differentiable on $\mathbb{U}$ and its Fréchet-derivative at the point $u \in \mathbb{U}$ is given by

$$
\frac{\mathrm{d} f_{B(u)}}{\mathrm{d} u}[h]=f_{B(u)}^{\prime}[B(h)] \quad \text { for all } \quad h \in L^{2}\left([0, T] ; \mathbb{R}^{N}\right) .
$$

The Fréchet-derivative depends Hölder-continuously on u, i.e., there exists some constant $C>0$ depending only on $\stackrel{\circ}{f}, T, K$ and $\beta$ such that

$$
\left\|f_{B\left(u_{1}\right)}^{\prime}[B(h)]-f_{B\left(u_{2}\right)}^{\prime}[B(h)]\right\|_{L^{2}\left(0, T ; L^{2}\right)} \leq C\left\|u_{1}-u_{2}\right\|_{L^{2}\left([0, T] ; \mathbb{R}^{N}\right)}^{\gamma}
$$

for all $u_{1}, u_{2} \in \mathbb{U}$ and $h \in L^{2}\left([0, T] ; \mathbb{R}^{N}\right)$ where $\gamma$ is the constant from Lemma 10.

For brevity we will use the notation $f_{u}:=f_{B(u)}$ and $f_{u}^{\prime}[h]:=f_{B(u)}^{\prime}[B(h)]$ for any $u \in \mathbb{U}$ and $h \in L^{2}\left([0, T] ; \mathbb{R}^{N}\right)$.

Proof For any $i \in\{1, \ldots, N\}$ the set $\mathbb{U}_{i} \subset L^{2}([0, T])$ is evidently bounded, convex and closed. Thus weak compactness follows directly from the theorems of Banach-Alaoglu and Mazur. The same holds for $\mathbb{U} \subset L^{2}\left([0, T] ; \mathbb{R}^{N}\right)$ which proves (a). The operator $B(\cdot)$ is obviously linear and for all $u \in L^{2}\left([0, T] ; \mathbb{R}^{N}\right)$,

$$
\begin{aligned}
\|B(u)\|_{L^{2}\left(0, T ; W^{2, \beta}\right)} & \leq \sum_{i=1}^{N}\left\|u_{i}\right\|_{L^{2}([0, T])}\left\|m_{i}\right\|_{W^{2, \beta}} \leq M \sum_{i=1}^{N}\left\|u_{i}\right\|_{L^{2}([0, T])} \\
& \leq M \sqrt{N}\left(\sum_{i=1}^{N}\left\|u_{i}\right\|_{L^{2}([0, T])}^{2}\right)^{1 / 2}=M \sqrt{N}\|u\|_{L^{2}\left([0, T] ; \mathbb{R}^{N}\right)} .
\end{aligned}
$$

Hence $B(\cdot)$ is continuous. Moreover this yields

$$
\|B(u)\|_{L^{2}\left(0, T ; W^{2, \beta}\right)}<M \sqrt{N}\left(\|a\|_{L^{2}\left([0, T] ; \mathbb{R}^{N}\right)}+\|b\|_{L^{2}\left([0, T] ; \mathbb{R}^{N}\right)}\right)=: \frac{K}{2}, \quad u \in \mathbb{U}
$$

and thus $B(\mathbb{U}) \subset \stackrel{\circ}{\mathbb{B}}_{K / 2}$. This proves (b) which directly implies (c). Finally (d) follows directly from Theorem 27, (b), (c) and the chain rule. 
We will now consider the following optimization problem with $\lambda_{i} \geq 0, i=1, \ldots, N$ and $\stackrel{\circ}{f}, f_{d} \in C_{c}^{2}\left(\mathbb{R}^{6}\right)$ such that $\|\stackrel{\circ}{f}\|_{p}=\left\|f_{d}\right\|_{p}$ for all $p \in[1, \infty]$ :

$$
\begin{aligned}
\text { Minimize } & I(f, u)=\frac{1}{2}\left\|f(T)-f_{d}\right\|_{L^{2}\left(\mathbb{R}^{6}\right)}^{2}+\sum_{i=1}^{N} \frac{\lambda_{i}}{2}\left\|u_{i}\right\|_{L^{2}([0, T])}^{2} \\
\text { s.t. } & \bullet u \in \mathbb{U} \\
& \bullet \quad B=B(u) \in \mathbb{B}_{K} \\
& -f \text { is a strong solution of the Vlasov-Poisson system } \\
& \partial_{t} f+v \cdot \partial_{x} f-\partial_{x} \psi_{f} \cdot \partial_{v} f+(v \times B) \cdot \partial_{v} f=0,\left.\quad f\right|_{t=0}=f \\
& \text { to the control } B .
\end{aligned}
$$

Using the control-state operator this problem can be reduced to

$$
\begin{array}{ll}
\text { Minimize } & J(u)=\frac{1}{2}\left\|f_{u}(T)-f_{d}\right\|_{L^{2}\left(\mathbb{R}^{6}\right)}^{2}+\sum_{i=1}^{N} \frac{\lambda_{i}}{2}\left\|u_{i}\right\|_{L^{2}([0, T])}^{2} \\
\text { s.t. } & u \in \mathbb{U} .
\end{array}
$$

\subsubsection{Existence of a globally optimal solution}

First we must show that this optimization has at least one solution:

Theorem 41 The optimization problem (6.24) possesses a globally optimal solution $\bar{u}$, i.e., for all $u \in \mathbb{U}, J(\bar{u}) \leq J(u)$. In this case it holds that

$$
\left\|\bar{u}_{i}\right\|_{L^{2}([0, T])} \leq \frac{2}{\sqrt{\lambda_{i}}}\|\stackrel{\circ}{f}\|_{L^{2}\left(\mathbb{R}^{6}\right)}, \quad i=1, \ldots, N
$$

Proof $J$ is bounded from below since $J(u) \geq 0$ for all $u \in \mathbb{U}$. Hence $M:=\inf _{u \in \mathbb{U}} J(u)$ exists and there also exists a minimizing sequence $\left(u_{k}\right)_{k \in \mathbb{N}} \subset \mathbb{U}$ such that $J\left(u_{k}\right) \rightarrow M$ if $k \rightarrow \infty$. As $\mathbb{U}$ is weakly compact this yields $u_{k} \rightarrow \bar{u}$ in $L^{2}\left([0, T] ; \mathbb{R}^{N}\right)$ for some weak limit $\bar{u} \in \mathbb{U}$ after extraction of a subsequence. Thus we also have $\left[u_{k}\right]_{i} \rightarrow \bar{u}_{i}$ in $L^{2}([0, T])$ for every $i \in\{1, \ldots, N\}$ and $B\left(u_{k}\right) \rightarrow B(\bar{u}) \in \mathbb{B}_{K}$ in $L^{2}\left(0, T ; W^{2, \beta}\right)$. From Proposition 22 we can conclude that $f_{u_{k}} \rightarrow f_{\bar{u}}$ in $W^{1,2}\left(0, T ; L^{2}\right)$ up to a subsequence. Then for any $\varphi \in L^{2}\left(\mathbb{R}^{6}\right)$

$$
\begin{gathered}
\int\left(f_{u_{k}}(T, z)-f_{\bar{u}}(T, z)\right) \varphi(z) \mathrm{d} z=\iint_{0}^{T} \frac{\mathrm{d}}{\mathrm{d} t}\left(f_{u_{k}}(t, z)-f_{\bar{u}}(t, z)\right) \mathrm{d} t \varphi(z) \mathrm{d} z \\
=\int_{0}^{T} \int\left(\partial_{t} f_{u_{k}}(t, z)-\partial_{t} f_{\bar{u}}(t, z)\right) \mathbb{1}_{[0, T]}(t) \varphi(z) \mathrm{d} z \mathrm{~d} t \rightarrow 0, \quad k \rightarrow 0,
\end{gathered}
$$

i.e., $f_{u_{k}}(T) \rightarrow f_{\bar{u}}(T)$ in $L^{2}\left(\mathbb{R}^{6}\right)$. 
Hence we can deduce from the weak lower semicontinuity of the $L^{2}$-norm that

$$
\begin{aligned}
J(\bar{u}) & =\frac{1}{2}\left\|f_{\bar{u}}(T)-f_{d}\right\|_{L^{2}}^{2}+\sum_{i=1}^{N} \frac{\lambda_{i}}{2}\left\|\bar{u}_{i}\right\|_{L^{2}}^{2} \\
& \leq \liminf _{k \rightarrow \infty}\left[\frac{1}{2}\left\|f_{u_{k}}(T)-f_{d}\right\|_{L^{2}}^{2}\right]+\sum_{i=1}^{N} \frac{\lambda_{i}}{2} \liminf _{k \rightarrow \infty}\left\|\left[u_{k}\right]_{i}\right\|_{L^{2}}^{2} \\
& \leq \liminf _{k \rightarrow \infty}\left[\frac{1}{2}\left\|f_{u_{k}}(T)-f_{d}\right\|_{L^{2}}^{2}+\sum_{i=1}^{N} \frac{\lambda_{i}}{2}\left\|\left[u_{k}\right]_{i}\right\|_{L^{2}}^{2}\right]=\lim _{k \rightarrow \infty} J\left(u_{k}\right)=M .
\end{aligned}
$$

By the definition of infimum this yields $J(\bar{u})=M$. Let us now assume that there exists some $i \in\{1, \ldots, N\}$ such that $\left\|\bar{u}_{i}\right\|_{L^{2}([0, T])}>\left(2 / \sqrt{\lambda_{i}}\right)\|\stackrel{\circ}{f}\|_{L^{2}\left(\mathbb{R}^{6}\right)}$. Then

$$
\begin{aligned}
J(\bar{u}) & \geq \frac{\lambda_{i}}{2}\left\|\bar{u}_{i}\right\|_{L^{2}([0, T])}^{2}>\frac{1}{2}\left(2\|\stackrel{\circ}{ }\|_{L^{2}\left(\mathbb{R}^{6}\right)}\right)^{2}=\frac{1}{2}\left(\left\|f_{0}(T)\right\|_{L^{2}}+\left\|f_{d}\right\|_{L^{2}}\right)^{2} \\
& \geq \frac{1}{2}\left\|f_{0}(T)-f_{d}\right\|_{L^{2}}^{2}=J(0)
\end{aligned}
$$

where 0 denotes the null function $u=(0, \ldots, 0) \in \mathbb{U}$. This, however, is a contradiction to the global optimality of $\bar{u}$ and thus the asserted inequality follows.

\subsubsection{Necessary conditions for local optimality}

Since our set of admissible controls is a box-restricted subset of $L^{2}\left([0, T] ; \mathbb{R}^{N}\right)$ this provides better possibilities to establish necessary optimality conditions compared to the model in the previous section. As the basic approach will be quite similar we will also have to discuss the costate equation:

Proposition 42 Let $u \in L^{2}\left([0, T] ; \mathbb{R}^{N}\right)$ be arbitrary and let $f_{u}=f_{B(u)}$ be its induced state that is given by the control-state operator. Moreover suppose that $\chi \in C_{c}^{2}\left(\mathbb{R}^{6} ;[0,1]\right)$ with $\chi=1$ on $B_{R_{Z}}(0)$. Then the the costate equation

$$
\left\{\begin{array}{l}
\partial_{t} g+v \cdot \partial_{x} g-\partial_{x} \psi_{f_{u}} \cdot \partial_{v} g+(v \times B(u)) \cdot \partial_{v} g=\Phi_{f_{u}, g} \chi \\
\left.g\right|_{t=T}=f_{\bar{u}}(T)-f_{d}
\end{array}\right.
$$

has a unique strong solution $g_{u} \in W^{1,2}\left(0, T ; C_{b}\right) \cap C\left([0, T] ; C_{b}^{1}\left(\mathbb{R}^{6}\right)\right) \cap L^{\infty}\left(0, T ; H^{2}\left(\mathbb{R}^{6}\right)\right)$ with supp $g_{u}(t) \subset B_{R^{*}}(0), t \in[0, T]$ for some constant $R^{*}>0$ depending only on $\stackrel{\circ}{f}, f_{d}, T, K$ and $\beta$.

In this case $\left.g_{u}\right|_{B_{R}(0)}$ does not depend on the choice of $\chi$ as long as $\chi=1$ on $B_{R_{Z}}(0)$.

Moreover $g_{u}$ depends Hölder-continuously on $u$ in such a way that there exists some constant $C \geq 0$ depending only on $\stackrel{\circ}{f} f_{d}, T, K, \beta$ and $\|\chi\|_{C_{b}^{1}}$ such that

$$
\left\|g_{u_{1}}-g_{u_{2}}\right\|_{W^{1,2}\left(0, T ; C_{b}\right)}+\left\|g_{u_{1}}-g_{u_{2}}\right\|_{C\left([0, T] ; C_{b}^{1}\right)} \leq C\left\|u_{1}-u_{2}\right\|_{L^{2}\left([0, T] ; \mathbb{R}^{N}\right)}^{\gamma}
$$

for all $u_{1}, u_{2} \in L^{2}\left([0, T] ; \mathbb{R}^{N}\right)$. 
Proof Since $u \in L^{2}\left([0, T] ; \mathbb{R}^{N}\right)$ and thus $B(u) \in \mathbb{B}_{K}$ this result follows directly from Theorem 31 and the estimate

$$
\left\|B\left(u_{1}\right)-B\left(u_{2}\right)\right\|_{L^{2}\left(0, T ; W^{2, \beta}\right)} \leq C\left\|u_{1}-u_{2}\right\|_{L^{2}\left([0, T] ; \mathbb{R}^{N}\right)}, \quad u_{1}, u_{2} \in L^{2}\left([0, T] ; \mathbb{R}^{N}\right)
$$

that is a direct consequence of Lemma $40(\mathrm{~b})$.

Of course the costate equation (6.25) does not appear out of thin air. Later, in the proof of Theorem 43, this equation will be deduced by Lagrangian technique.

In the previous section it was only possible to obtain optimality conditions for inner points of the set $\mathbb{B}_{K}$. Here, as $\mathbb{U}$ is a box-restricted subset of $L^{2}$, the optimality conditions can be established on the whole set $\mathbb{U}$. This is essential because a discussion of the inner points of $\mathbb{U}$ would not make any sense as the interior of $\mathbb{U}$ is empty. The following theorem provides a list of equivalent necessary conditions for local optimality:

Theorem 43 Suppose that $\lambda_{i}>0$ for every $i \in\{1, \ldots, N\}$ and let $\bar{u} \in \mathbb{U}$ be any function. According to the definition of the control-state operator $f_{\bar{u}}$ denotes the unique strong solution of the state equation to the field $B(\bar{u}) \in \mathbb{B}_{K}$. Moreover let $g_{\bar{u}}$ denote the unique strong solution of the costate equation (6.25). We define the function $p(\bar{u}):[0, T] \rightarrow \mathbb{R}^{N}$ by $p(\bar{u})=\left(p_{1}(\bar{u}), \ldots, p_{N}(\bar{u})\right)^{T}$ with

$$
p_{i}(u)(t):=\int\left(v \times m_{i}(x)\right) \cdot \partial_{v} f_{u}(t, x, v) g_{u}(t, x, v) \mathrm{d}(x, v), \quad i=1, \ldots, N .
$$

For every $\bar{u} \in L^{2}\left([0, T] ; \mathbb{R}^{N}\right), p(\bar{u}) \in C\left([0, T] ; \mathbb{R}^{N}\right)$.

Then the following items are equivalent:

(i) $\bar{u}$ satisfies the variation inequality, i.e., for all $u=\left(u_{1}, \ldots, u_{N}\right) \in \mathbb{U}$,

$$
\int_{0}^{T}\left(\lambda_{i} \bar{u}_{i}-p_{i}(\bar{u})\right)\left(u_{i}-\bar{u}_{i}\right) \mathrm{d} t \geq 0, \quad i=1, \ldots, N .
$$

(ii) For almost every $t \in[0, T]$ and every $i \in\{1, \ldots, N\}$,

$$
\bar{u}_{i}(t)= \begin{cases}a_{i}(t), & \text { if } \lambda_{i} \bar{u}_{i}(t)-p_{i}(\bar{u})(t)>0 \\ \in\left[a_{i}(t), b_{i}(t)\right], & \text { if } \lambda_{i} \bar{u}_{i}(t)-p_{i}(\bar{u})(t)=0 \\ b_{i}(t), & \text { if } \lambda_{i} \bar{u}_{i}(t)-p_{i}(\bar{u})(t)<0\end{cases}
$$

where $\bar{u}$ is an arbitrary but fixed representative of its equivalence class.

(iii) $\bar{u}$ satisfies the pointwise variation inequality, i.e., for almost all $t \in[0, T]$ and any $i \in\{1, \ldots, N\}$,

$$
\left(\lambda_{i} \bar{u}_{i}(t)-p_{i}(\bar{u})(t)\right)\left(w-\bar{u}_{i}(t)\right) \geq 0, \quad w \in\left[a_{i}(t), b_{i}(t)\right] .
$$


In other words $\bar{u}$ satisfies the weak minimum principle, i.e., for almost all $t \in[0, T] \times \mathbb{R}^{3}$ and any $i \in\{1, \ldots, N\}$,

$$
\min _{w \in\left[a_{i}(t), b_{i}(t)\right]}\left(\lambda_{i} \bar{u}_{i}(t)-p_{i}(\bar{u})(t)\right) w=\left(\lambda_{i} \bar{u}_{i}(t)-p_{i}(\bar{u})(t)\right) \bar{u}_{i}(t) .
$$

(iv) $\bar{u}$ satisfies the (strong) minimum principle, i.e., for almost all $t \in[0, T]$ and any $i \in\{1,2,3\}$,

$$
\min _{w \in\left[a_{i}(t), b_{i}(t)\right]}\left[\frac{1}{2} \lambda_{i} w^{2}-p_{i}(\bar{u})(t) w\right]=\left[\frac{1}{2} \lambda_{i} \bar{u}_{i}(t)^{2}-p_{i}(\bar{u})(t) \bar{u}_{i}(t)\right] .
$$

(v) $\bar{u}$ is given implicitely by the projection formula, i.e., for almost all $t \in[0, T]$ and any $i \in\{1,2,3\}$,

$$
\bar{u}_{i}(t)=\mathbb{P}_{\left[a_{i}(t), b_{i}(t)\right]}\left(\frac{1}{\lambda_{i}} p_{i}(\bar{u})(t)\right)
$$

where $\mathbb{P}_{[a, b]}$ denotes the projection of $\mathbb{R}$ onto the interval $[a, b]$, i.e.,

$$
\mathbb{P}_{[a, b]}(w)=\min \{b, \max \{a, w\}\}, \quad w \in \mathbb{R} .
$$

Now suppose that $\bar{u}$ is a locally optimal solution of the optimization problem (6.24), i.e., there exists $\delta>0$ such that $J(\bar{u}) \leq J(u)$ for every $u \in \mathbb{U}$ with $\|\bar{u}-u\|_{L^{2}}<\delta$. Then $\bar{u}$ satisfies the assertions (i)-(v). This means that these items are necessary conditions for local optimality.

\section{Comment}

(a) We can establish similar results if $\lambda_{i}=0$. Actually the items (i)-(iv) stay true in this case if we just replace $\lambda_{i}$ by zero. Instead of (v) we only have

$$
\bar{u}_{i}(t)= \begin{cases}a_{i}(t), & \text { if } p_{i}(\bar{u})(t)>0 \\ b_{i}(t), & \text { if } p_{i}(\bar{u})(t)<0\end{cases}
$$

but $\bar{u}_{i}$ is undefined if $p_{i}(\bar{u})(t)=0$. This phenomenon is called a bang-bang control as it switches abruptly between the two boundary functions.

(b) If $a_{i}$ and $b_{i}$ are continuous, so is $\bar{u}_{i}$ due to item (v). If this holds for all $i \in\{1, \ldots, N\}$ we know that $\bar{u}$ is continuous and consequently $B(\bar{u}) \in C\left([0, T] ; C^{1, \gamma}\right)$. In this case $f_{\bar{u}}$ and $g_{\bar{u}}$ are classical solutions of their respective systems.

Proof The assertion $p_{i}(\bar{u}) \in C([0, T])$ is obvious since $f_{\bar{u}}$ and $g_{\bar{u}}$ are in $C\left([0, T] ; C_{b}^{1}\right)$. First we will show that item (i) holds if $\bar{u}$ is a locally optimal solution. Therefore we will approach similarly to the previous section and apply the Lagrangian technique: 
For $u \in \mathbb{U}$ and $f, g \in H^{1}(] 0, T\left[\times \mathbb{R}^{6}\right)$ with supp $f(t) \subset B_{R}(0)$ for all $t \in[0, T]$ we define the Lagrangian

$$
\begin{aligned}
\mathcal{L}(f, u, g):= & \frac{1}{2}\left\|f(T)-f_{d}\right\|_{L^{2}}+\sum_{i=1}^{N} \frac{\lambda_{i}}{2}\left\|u_{i}\right\|_{L^{2}}^{2} \\
& -\int_{[0, T] \times \mathbb{R}^{6}}\left(\partial_{t} f+v \cdot \partial_{x} f-\partial_{x} \psi_{f} \cdot \partial_{v} f+(v \times B(u)) \cdot \partial_{v} f\right) g \mathrm{~d}(t, x, v) .
\end{aligned}
$$

Integration by parts yields

$$
\begin{aligned}
\mathcal{L}(f, u, g)= & \frac{1}{2}\left\|f(T)-f_{d}\right\|_{L^{2}}+\sum_{i=1}^{N} \frac{\lambda_{i}}{2}\left\|u_{i}\right\|_{L^{2}}^{2}+\langle g(0), f(0)\rangle_{L^{2}}-\langle g(T), f(T)\rangle_{L^{2}} \\
& +\int_{[0, T] \times \mathbb{R}^{6}}\left(\partial_{t} g+v \cdot \partial_{x} g-\partial_{x} \psi_{f} \cdot \partial_{v} g+(v \times B(u)) \cdot \partial_{v} g\right) f \mathrm{~d}(t, x, v) .
\end{aligned}
$$

The Lagrangian is partially Fréchet differentiable with

$$
\begin{aligned}
\left(\partial_{f} \mathcal{L}\right)(f, u, g)[h]= & \left\langle f(T)-f_{d}, h(T)\right\rangle_{L^{2}}-\langle g(T), h(T)\rangle_{L^{2}}+\langle g(0), h(0)\rangle_{L^{2}} \\
& +\int_{[0, T] \times \mathbb{R}^{6}}\left(\partial_{t} g+v \cdot \partial_{x} g-\partial_{x} \psi_{f} \cdot \partial_{v} g+(v \times B(u)) \cdot \partial_{v} g\right) h \mathrm{~d}(t, x, v) \\
& -\int_{[0, T] \times \mathbb{R}^{6}} \Phi_{f, g}(t, x) h \mathrm{~d}(t, x, v)
\end{aligned}
$$

for any $h \in H^{1}(] 0, T\left[\times \mathbb{R}^{6}\right)$ and

$$
\begin{aligned}
& \left(\partial_{u} \mathcal{L}\right)(f, u, g)[h]=\sum_{i=1}^{N} \lambda_{i}\left\langle u_{i}, h_{i}\right\rangle_{L^{2}}-\int_{[0, T] \times \mathbb{R}^{6}}(v \times B(h)) \cdot \partial_{v} f g \mathrm{~d}(t, x, v) \\
& =\int_{0}^{T} \sum_{i=1}^{N} \lambda_{i} u_{i} h_{i} \mathrm{~d} t-\int_{0}^{T} \sum_{i=1}^{N} \int_{\mathbb{R}^{6}}\left(v \times m_{i}\right) \cdot \partial_{v} f g \mathrm{~d}(x, v) h_{i}(t) \mathrm{d} t \\
& =\sum_{i=1}^{N} \int_{0}^{T}\left(\lambda_{i} u_{i}(t)-p_{i}(\bar{u})(t)\right) h_{i}(t) \mathrm{d} t
\end{aligned}
$$

for any $h \in L^{2}\left([0, T] ; \mathbb{R}^{N}\right)$. Obviously $J(\bar{u})=\mathcal{L}\left(f_{\bar{u}}, \bar{u}, g\right)$ and hence

$$
J^{\prime}(\bar{u})[h]=\left(\partial_{f} \mathcal{L}\right)\left(f_{\bar{u}}, \bar{u}, g\right)\left[f_{\bar{u}}^{\prime}[h]\right]+\left(\partial_{B} \mathcal{L}\right)\left(f_{\bar{u}}, \bar{u}, g\right)[h], \quad h \in L^{2}\left([0, T] ; \mathbb{R}^{N}\right) .
$$

Recall that $J^{\prime}(\bar{u})[h]$ is nonnegative if $\bar{u}$ is a local minimizer of $J$. Thus inserting $g=g_{\bar{u}}$ yields

$$
\begin{aligned}
0 & \leq J^{\prime}(\bar{u}) h=\left(\partial_{f} \mathcal{L}\right)\left(f_{\bar{u}}, \bar{u}, g_{\bar{u}}\right)\left[f_{\bar{u}}^{\prime}[h]\right]+\left(\partial_{u} \mathcal{L}\right)\left(f_{\bar{u}}, \bar{u}, g_{\bar{u}}\right)[h] \\
& =\sum_{i=1}^{N} \int_{0}^{T}\left(\lambda_{i} \bar{u}_{i}(t)-p_{i}(\bar{u})(t)\right) h_{i}(t) \mathrm{d} t
\end{aligned}
$$


for all $h \in L^{2}\left([0, T] ; \mathbb{R}^{N}\right)$. For any fixed $i \in\{1, \ldots, N\}$ we can choose $h_{j}=0$ if $j \neq i$ while $h_{i}$ is still arbitrary. This finally implies that

$$
\int_{0}^{T}\left(\lambda_{i} \bar{u}_{i}-p_{i}(\bar{u})\right) h_{i} \mathrm{~d} t \geq 0, \quad i=1, \ldots, N
$$

for all $h \in L^{2}\left([0, T] ; \mathbb{R}^{N}\right)$ with $u+h \in \mathbb{U}$. For any arbitrary $u \in \mathbb{U}$ we can now choose $h:=u-\bar{u} \in L^{2}\left([0, T] ; \mathbb{R}^{N}\right)$ and hence we can conclude that for all $u \in \mathbb{U}$,

$$
\int_{0}^{T}\left(\lambda_{i} \bar{u}_{i}-p_{i}(\bar{u})\right)\left(u_{i}-\bar{u}_{i}\right) \mathrm{d} t \geq 0, \quad i=1, \ldots, N .
$$

that is (i).

Now we will show that for any $\bar{u} \in \mathbb{U}$ the items (i)-(v) are equivalent.

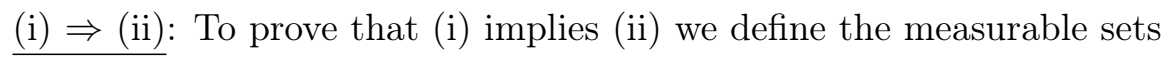

$$
\begin{aligned}
& A_{i}^{+}:=\left\{t \in[0, T] \mid \lambda_{i} \bar{u}_{i}(t)-p_{i}(\bar{u})(t)>0\right\}, \\
& A_{i}^{-}:=\left\{t \in[0, T] \mid \lambda_{i} \bar{u}_{i}(t)-p_{i}(\bar{u})(t)<0\right\}, \\
& A_{i}^{0}:=\left\{t \in[0, T] \mid \lambda_{i} \bar{u}_{i}(t)-p_{i}(\bar{u})(t)=0\right\}
\end{aligned}
$$

for $i=1,2,3$ where $\bar{u}$ denotes an arbitrary but fixed representative of its equivalence class. Let now $i \in\{1,2,3\}$ be arbitrary. We assume that there exists some measurable subset $E^{+} \subset A_{i}^{+}$such that $\bar{u}_{i}>a_{i}$ almost everywhere on $E^{+}$or some measurable subset $E^{-} \subset A_{i}^{-}$such that $\bar{u}_{i}<b_{i}$ on $E^{-}$. In the first case we choose $u \in \mathbb{U}$ such that

$$
u_{i}(t)= \begin{cases}a_{i}(t), & \text { if } t \in E^{+} \\ \bar{u}_{i}(t), & \text { else }\end{cases}
$$

Then

$$
\int_{0}^{T}\left(\lambda_{i} \bar{u}_{i}-p_{i}(\bar{u})\right)\left(u_{i}-\bar{u}_{i}\right) \mathrm{d} t=\int_{E^{+}}\left(\lambda_{i} \bar{u}_{i}-p_{i}(\bar{u})\right)\left(a_{i}-\bar{u}_{i}\right) \mathrm{d} t<0
$$

which is a contradiction to (i). The other case can be treated analogously. Hence $\bar{u}_{i}=a_{i}$ on $A_{i}^{+}(\bar{u})$ and $\bar{u}_{i}=b_{i}$ on $A_{i}^{-}(\bar{u})$ that is (ii).

(ii) $\Rightarrow$ (iii): As $\bar{u}_{i}=a_{i}$ almost everywhere on $A_{i}^{+}(\bar{u})$ we can easily conclude that for $\overline{\text { almost all } t} t \in A_{i}^{+}(\bar{u})$ we have $w-\bar{u}_{i}(t) \geq 0$ for any real number $w \in\left[a_{i}(t, x), b_{i}(t, x)\right]$. Hence the pointwise variation inequality holds almost everywhere on $A_{i}^{+}$. We can show similarly that this inequality also holds almost everywhere on $A_{i}^{-}$. Obviously the inequality remains correct almost everywhere on $A_{i}^{0}$ because $\lambda_{i} \bar{u}_{i}-p_{i}(\bar{u})$ is vanishing almost everywhere on this set. The weak minimum principle is only a reformulation of the pointwise variation inequality.

(iii) $\Rightarrow$ (iv): Let now $t \in[0, T]$ be any point where the pointwise variation inequality 
holds. We consider the continuously differentiable function

$$
j:\left[a_{i}(t), b_{i}(t)\right] \rightarrow \mathbb{R}, \quad w \mapsto \frac{\lambda_{i}}{2} w^{2}-p_{i}(\bar{u})(t) w .
$$

As the interval $\left[a_{i}(t), b_{i}(t)\right]$ is compact there exists $\bar{w} \in\left[a_{i}(t), b_{i}(t)\right]$ such that

$$
\bar{w}=\min _{w \in\left[a_{i}(t), b_{i}(t)\right]} j(w) .
$$

Of course the minimizer $\bar{w}$ is unique since $j$ is strictly convex. This means that $\bar{w}$ is the minimizer of $j$ on $\left[a_{i}(t), b_{i}(t)\right]$ iff

$$
0 \leq j^{\prime}(\bar{w})(w-\bar{w})=\left(\lambda_{i} \bar{w}-p_{i}(\bar{u})(t)\right)(w-\bar{w}), \quad w \in\left[a_{i}(t, x), b_{i}(t, x)\right] .
$$

Hence we can conclude from the pointwise variation inequality that $\bar{w}$ is the unique minimizer of $j$ on the interval $\left[a_{i}(t), b_{i}(t)\right]$ if and only if $\bar{w}=\bar{u}_{i}(t)$. This implies (iv) as $t$ was arbitrary.

(iv) $\Rightarrow(\mathrm{v})$ : Since $j^{\prime}(\bar{w})=\lambda_{i} \bar{w}-p_{i}(\bar{u})(t)$ it holds that

$$
\begin{array}{lll}
\lambda_{i} \bar{w}-p_{i}(\bar{u})(t) \geq 0 & \text { iff } & \bar{w}=a_{i}(t), \\
\lambda_{i} \bar{w}-p_{i}(\bar{u})(t)=0 & \text { iff } & \bar{w} \in] a_{i}(t), b_{i}(t)[ \\
\lambda_{i} \bar{w}-p_{i}(\bar{u})(t) \leq 0 & \text { iff } & \bar{w}=b_{i}(t)
\end{array}
$$

Consequently the minimizer $\bar{w}$ is uniquely determined by

$$
\bar{u}_{i}(t)=\bar{w}=\mathbb{P}_{\left[a_{i}(t), b_{i}(t)\right]}\left(\frac{1}{\lambda_{i}} p_{i}(\bar{u})(t)\right) .
$$

This proves (v).

(v) $\Rightarrow(\mathrm{i})$ : For any $i \in\{1, \ldots, N\}$ we can split the time interval into three disjoint measurable sets, i.e., $[0, T]=I_{+} \cup I_{0} \cup I_{-}$up to a nullset where

$$
\begin{aligned}
& I_{+}:=\left\{t \in[0, T] \mid p_{i}(\bar{u})(t) \leq \lambda_{i} a_{i}(t)\right\}, \\
& I_{0}:=\left\{t \in[0, T] \mid \lambda_{i} a_{i}(t)<p_{i}(\bar{u})(t)<\lambda_{i} b_{i}(t)\right\}, \\
& I_{-}:=\left\{t \in[0, T] \mid p_{i}(\bar{u})(t) \geq \lambda_{i} b_{i}(t)\right\} .
\end{aligned}
$$

Then

$$
\begin{aligned}
& \int_{0}^{T}\left(\lambda_{i} \bar{u}_{i}-p_{i}(\bar{u})\right)\left(u_{i}-\bar{u}_{i}\right) \mathrm{d} t \\
& =\int_{I_{+}}\left(\lambda_{i} a_{i}-p_{i}(\bar{u})\right)\left(u_{i}-a_{i}\right) \mathrm{d} t+\int_{I_{0}}\left(p_{i}(\bar{u})-p_{i}(\bar{u})\right)\left(u_{i}-\frac{1}{\lambda_{i}} p_{i}(\bar{u})\right) \mathrm{d} t \\
& \quad+\int_{I_{-}}\left(\lambda_{i} b_{i}-p_{i}(\bar{u})\right)\left(u_{i}-b_{i}\right) \mathrm{d} t \\
& \quad \geq 0
\end{aligned}
$$

that is (i). 
If $\bar{u} \in \mathbb{U}$ is a locally optimal control we can also show similarly to Proposition 33 that the triple $\left(f_{\bar{u}}, g_{\bar{u}}, \bar{u}\right)$ satisfies a certain system of partial differential equation that will be referred to as the optimality system of the optimization problem.

Definition 44 The triple $(f, g, u)$ is called a strong solution of the optimality system iff the following conditions hold:

(i) $f, g \in W^{1,2}\left(0, T ; C_{b}\right) \cap C\left([0, T] ; C_{b}^{1}\right)$ and $u \in L^{2}\left([0, T] ; \mathbb{R}^{N}\right)$.

(ii) For any $t \in[0, T]$,

$$
\operatorname{supp} f(t) \subset B_{R}(0) \quad \text { and } \quad \operatorname{supp} g(t) \subset B_{R^{*}}(0)
$$

where $R>0$ and $R^{*}>0$ are the constants from Theorem 13 and Theorem 42.

(iii) $f, g$ and $u$ satisfy the following system of equations almost everywhere:

$$
\left\{\begin{array}{l}
\partial_{t} f+v \cdot \partial_{x} f-\partial_{x} \psi_{f} \cdot \partial_{v} f+(v \times B(u)) \cdot \partial_{v} f=0 \\
\partial_{t} g+v \cdot \partial_{x} g-\partial_{x} \psi_{f} \cdot \partial_{v} g+(v \times B(u)) \cdot \partial_{v} g=\Phi_{f, g} \chi \\
u=\left(u_{1}, \ldots, u_{N}\right)^{T} \text { with } u_{i}=\mathbb{P}_{\left[a_{i}, b_{i}\right]}\left(\frac{1}{\lambda_{i}} \int\left(v \times m_{i}\right) \cdot \partial_{v} f g \mathrm{~d} v\right) .
\end{array}\right.
$$

(iv) $f$ and $g$ satisfy the following initial/final value condition:

$$
\left.f\right|_{t=0}=\stackrel{\circ}{f},\left.\quad g\right|_{t=T}=f(T)-f_{d} .
$$

Then Theorem $43(\mathrm{v})$ immediately yields the following result:

Corollary 45 Suppose that $\bar{u} \in \mathbb{U}$ is a locally optimal solution of the optimization problem (6.2). Then the triple $\left(f_{\bar{u}}, g_{\bar{u}}, \bar{u}\right)$ is a strong solution of the optimality system ((6.26), (6.27)).

Obviously the necessary optimality condition that is given by this corollary is equivalent to the items of Theorem 43.

\subsubsection{A sufficient condition for local optimality}

The derivation of sufficient conditions for local optimality is basically similar to the approach for the $\mathbb{B}_{K}$-fields. First of all we will also need Fréchet-differentiability of the costate. 
Lemma 46 Let g. : $L^{2}\left([0, T] ; \mathbb{R}^{N}\right) \rightarrow C\left([0, T] ; L^{2}\left(\mathbb{R}^{6}\right)\right), u \mapsto g_{u}$ denote the controlcostate operator. For any $u, h \in L^{2}\left([0, T] ; \mathbb{R}^{N}\right)$ with $B(u) \in \stackrel{\circ}{\mathbb{B}}_{K}$ there exists a unique strong solution $\left.g_{u}^{h} \in L^{\infty} \cap H^{1}(] 0, T\left[\times \mathbb{R}^{6}\right) \subset C\left([0, T] ; L^{2}\left(\mathbb{R}^{6}\right)\right)\right)$ of the final value problem

$$
\left\{\begin{array}{l}
\partial_{t} g+v \cdot \partial_{x} g-\partial_{x} \psi_{f_{u}^{\prime}[h]} \cdot \partial_{v} g_{u}-\partial_{x} \psi_{f_{u}} \cdot \partial_{v} g+(v \times B(u)) \cdot \partial_{v} g+(v \times B(h)) \cdot \partial_{v} g_{u} \\
\quad=\Phi_{f_{u}, g} \chi-\Phi_{g_{u}, f_{u}^{\prime}[h]} \chi \\
\left.g\right|_{t=T}=0 .
\end{array}\right.
$$

Recall that $f_{u}^{\prime}[h]$ denotes the derivative $f_{B(u)}^{\prime}[B(h)]$ of the control-state operator. Then the following holds:

(a) Let $t \in[0, T]$ be arbitrary. Then $g .(t)$ is Fréchet differentiable on $\mathbb{U}$ with respect to the $L^{2}\left(\mathbb{R}^{6}\right)$-norm, i.e., for any $u \in \mathbb{U}$ there exists a unique linear operator $g_{u}^{\prime}(t): L^{2}\left([0, T] ; \mathbb{R}^{N}\right) \rightarrow L^{2}\left(\mathbb{R}^{6}\right)$ such that

$$
\begin{gathered}
\forall \varepsilon>0 \exists \delta>0 \forall h \in L^{2}\left([0, T] ; \mathbb{R}^{N}\right) \text { with }\|h\|_{L^{2}}<\delta: \\
B(u+h) \in \mathbb{B}_{K} \quad \text { and } \quad \frac{\left\|g_{u+h}(t)-g_{u}(t)-g_{u}^{\prime}(t)[h]\right\|_{L^{2}}}{\|h\|_{L^{2}\left([0, T] ; \mathbb{R}^{N}\right)}}<\varepsilon .
\end{gathered}
$$

The Fréchet derivative is given by

$$
g_{u}^{\prime}(t)[h]=g_{u}^{h}(t), \quad h \in L^{2}\left([0, T] ; \mathbb{R}^{N}\right) .
$$

(b) The control-costate operator $g$. is Fréchet differentiable on $\mathbb{U}$ with respect to the $C\left([0, T] ; L^{2}\left(\mathbb{R}^{6}\right)\right)$-norm, i.e., for any $u \in \mathbb{U}$ there exists a unique linear operator $g_{u}^{\prime}: L^{2}\left([0, T] ; \mathbb{R}^{N}\right) \rightarrow C\left([0, T] ; L^{2}\left(\mathbb{R}^{6}\right)\right)$ such that

$$
\begin{gathered}
\forall \varepsilon>0 \exists \delta>0 \forall h \in L^{2}\left([0, T] ; \mathbb{R}^{N}\right) \text { with }\|h\|_{L^{2}}<\delta: \\
B(u+h) \in \mathbb{B}_{K} \quad \text { and } \quad \frac{\left\|g_{u+h}-g_{u}-g_{u}^{\prime}[h]\right\|_{C\left([0, T] ; L^{2}\right)}}{\|h\|_{L^{2}\left([0, T] ; \mathbb{R}^{N}\right)}}<\varepsilon .
\end{gathered}
$$

The Fréchet derivative is given by

$$
g_{u}^{\prime}[h]=g_{u}^{h}, \quad h \in L^{2}\left([0, T] ; \mathbb{R}^{N}\right) .
$$

(c) For all $h \in L^{2}\left([0, T] ; \mathbb{R}^{N}\right)$, the solution $g_{u}^{h}$ depends Hölder-continuously on $u \in \mathbb{U}$ in such a way that there exists some constant $C>0$ depending only on $\stackrel{\circ}{f}, T, K$ and $\beta$ such that

$$
\sup _{\|h\|_{L^{2}\left([0, T] ; \mathbb{R}^{N}\right)} \leq 1}\left\|g_{u_{1}}^{\prime}[h]-g_{u_{2}}^{\prime}[h]\right\|_{L^{2}\left(0, T ; L^{2}\right)} \leq C\left\|u_{1}-u_{2}\right\|_{L^{2}\left([0, T] ; \mathbb{R}^{N}\right)}^{\gamma}
$$

for all $u_{1}, u_{2} \in \mathbb{U}$ and $h \in L^{2}\left([0, T] ; \mathbb{R}^{N}\right)$.

The proof proceeds analogously to the proof of Theorem 27. Therefore it will not be presented. 
From this result we can conclude that the control-state operator is twice continuously Fréchet-differentiable.

Corollary 47 The cost functional $J$ of the optimization problem (6.2) is twice Fréchet differentiable on $\mathbb{U}$. The Fréchet derivative of second order at the point $u \in \mathbb{U}$ can be described as a bilinear operator $J^{\prime \prime}(u): L^{2}\left([0, T] ; \mathbb{R}^{N}\right)^{2} \rightarrow \mathbb{R}$ that is given by

$$
\begin{aligned}
& J^{\prime \prime}(u)[h, \tilde{h}] \\
& =\sum_{i=1}^{N}\left\{\lambda_{i}\left\langle h_{i}, \tilde{h}_{i}\right\rangle_{L^{2}([0, T])}-\int_{[0, T] \times \mathbb{R}^{6}}\left(v \times m_{i}\right) \cdot\left(\partial_{v} f_{u} g_{u}^{\prime}[\tilde{h}]-\partial_{v} g_{u} f_{u}^{\prime}[\tilde{h}]\right) h_{i} \mathrm{~d}(t, x, v)\right\}
\end{aligned}
$$

for all $h, \tilde{h} \in L^{2}\left([0, T] ; \mathbb{R}^{N}\right)$. Moreover there exists some constant $C>0$ depending only on $\stackrel{\circ}{f} f_{d}, K, T$ and $\beta$ such that for all $u, \tilde{u} \in \mathbb{U}$,

$$
\left\|J^{\prime \prime}(u)-J^{\prime \prime}(\tilde{u})\right\| \leq C\|u-\tilde{u}\|_{L^{2}\left([0, T] ; \mathbb{R}^{N}\right)}^{\gamma}
$$

where

$$
\left\|J^{\prime \prime}(u)\right\|=\sup \left\{\left|J^{\prime \prime}(u)\left[h_{1}, h_{2}\right]\right| \mid\left\|h_{1}\right\|_{L^{2}\left([0, T] ; \mathbb{R}^{N}\right)}=1,\left\|h_{2}\right\|_{L^{2}\left([0, T] ; \mathbb{R}^{N}\right)}=1\right\}
$$

denotes the operator norm. This means that $J$ is twice continuously differentiable.

Finally we obtain a sufficient condition for local optimality:

Theorem 48 Suppose that $\bar{u} \in \mathbb{B}_{K}$ and let $f_{\bar{u}}$ and $g_{\bar{u}}$ be its induced state and costate. Let $0<\alpha<2+\gamma$ be any real number. We assume that the variation inequality

$$
\int_{0}^{T}\left(\lambda_{i} \bar{u}_{i}-p_{i}(\bar{u})\right)\left(u_{i}-\bar{u}_{i}\right) \mathrm{d} t \geq 0, \quad i=1, \ldots, N
$$

holds for all $u \in \mathbb{U}$ and there exists some $\varepsilon>0$ such that for all $h \in L^{2}\left([0, T] ; \mathbb{R}^{N}\right)$,

$$
\begin{aligned}
& \sum_{i=1}^{N}\left\{\lambda_{i}\left\|h_{i}\right\|_{L^{2}([0, T])}^{2}-\int_{[0, T] \times \mathbb{R}^{6}}\left(v \times m_{i}\right) \cdot\left(\partial_{v} f_{\bar{u}} g_{\bar{u}}^{\prime}[h]-\partial_{v} g_{\bar{u}} f_{\bar{u}}^{\prime}[h]\right) h_{i} \mathrm{~d}(t, x, v)\right\} \\
& \quad \geq \varepsilon\|h\|_{L^{2}\left([0, T] ; \mathbb{R}^{N}\right)}^{\alpha} .
\end{aligned}
$$

In this case $J$ satisfies the following growth condition: There exist $\delta>0$ such that for all $u \in \mathbb{U}$ with $\|u-\bar{u}\|_{L^{2}\left([0, T] ; \mathbb{R}^{N}\right)}<\delta$,

$$
J(u) \geq J(\bar{u})+\frac{\varepsilon}{4}\|u-\bar{u}\|_{L^{2}\left([0, T] ; \mathbb{R}^{N}\right)}^{\alpha}
$$

and hence $\bar{u}$ is even a strict local minimizer of $J$ on the set $\mathbb{U}$.

The proofs of the above results are analogous to those of Corollary 35 and Theorem 36 . 


\subsubsection{Uniqueness of the optimal solution on small time intervals}

Theorem 49 Let $\lambda>0$ be defined by $\lambda:=\min \left\{\lambda_{1}, \ldots, \lambda_{N}\right\}$. Suppose that $\left.\left.\lambda \in\right] 0,1\right]$ and let us assume that there exists a strong solution $(f, g, u)$ of the optimality system $((6.26),(6.27))$. Then this solution is unique if the quotient $\frac{T}{\lambda}$ is sufficiently small.

Proof Suppose that the triple $(\tilde{f}, \tilde{g}, \tilde{u})$ is another strong solution. Let $C=C(T) \geq 0$ denote some generic constant that may depend on $T, a, b, \stackrel{\circ}{f}, f_{d}$ and the $C\left([0, T] ; C_{b}^{1}\right)$ norm of $f, \tilde{f}, g$ and $\tilde{g}$. We can assume that $C=C(T)$ is monotonically increasing in $T$. Then for almost all $t \in[0, T]$,

$$
\begin{aligned}
\left|u_{i}(t)-\tilde{u}_{i}(t)\right| & =\left|\mathbb{P}_{\left[a_{i}(t), b_{i}(t)\right]}\left(\frac{1}{\lambda_{i}} p_{u_{i}}(t)\right)-\mathbb{P}_{\left[a_{i}(t), b_{i}(t)\right]}\left(\frac{1}{\lambda_{i}} p_{\tilde{u}_{i}}(t)\right)\right| \\
& \leq \frac{1}{\lambda_{i}}\left|p_{u_{i}}(t)-p_{\tilde{u}_{i}}(t)\right| \\
& \leq \frac{C}{\lambda}\left\|f_{u}(t)-f_{\tilde{u}}(t)\right\|_{\infty}+\frac{C}{\lambda}\left\|g_{u}(t)-g_{\tilde{u}}(t)\right\|_{\infty}
\end{aligned}
$$

and hence

$$
\|B(u)(t)-B(\tilde{u})(t)\|_{\infty} \leq \frac{C}{\lambda}\left\|f_{u}(t)-f_{\tilde{u}}(t)\right\|_{\infty}+\frac{C}{\lambda}\left\|g_{u}(t)-g_{\tilde{u}}(t)\right\|_{\infty} .
$$

The rest proceeds analogously to the proof of Theorem 37 .

Finally, we can easily deduce uniqueness of the globally optimal solution if $\frac{T}{\lambda}$ is small:

Corollary 50 Let $\lambda>0$ be the constant from Theorem 49 and let $\bar{u} \in \mathbb{U}$ be a locally optimal solution of the optimization problem (6.24). Then the tripel $\left(f_{\bar{u}}, g_{\bar{u}}, \bar{u}\right)$ is a strong solution of the optimality system ((6.26), (6.27)) according to Corollary 45.

If now $\lambda \in] 0,1]$ and $\frac{T}{\lambda}$ is sufficiently small then $\bar{u}$ is the only locally optimal solution of the optimization problem (6.24).

In this case $\bar{u}$ is also the unique globally optimal solution of the optimization problem (6.24) and the items of Theorem 43 are necessary and sufficient conditions for global optimality.

Proof If $\lambda \in] 0,1]$ and $\frac{T}{\lambda}$ is sufficiently small then Theorem 49 and Corollary 45 ensure that $\bar{u}$ is the only locally optimal solution in $\mathbb{U}$. Recall that there exists at least one globally optimal solution $u^{*}$ according to Theorem 41 . As any globally optimal solution is also locally optimal it follows that $u^{*}=\bar{u}$. Hence there is exactly one globally optimal solution and this solution must be $\bar{u}$. As the assertion of Corollary 45 is equivalent to the items of Theorem 43 those items are necessary and sufficient conditions for global optimality. 


\section{Appendix}

Proof of Lemma 15 Let $s, t \in[0, T]$ and $z \in B_{R}(0)$ be arbitrary (without loss of generality $s \leq t)$ and let $i, j \in 1, \ldots, 6$ be arbitrary indices. Let $B \in \mathbb{M}$ be an arbitrary field and let $Z_{B}:[0, T] \times[0, T] \times \mathbb{R}^{6} \rightarrow \mathbb{R}^{6}$ denote the induced solution of the characteristic system satisfying the initial condition $Z_{B}(t, t, z)=z$. For brevity, we will use the notation $Z_{B}(s)=Z_{B}(s, t, z)$. The letter $C$ will denote a positive generic constant depending only on $\stackrel{\circ}{f} K, T$ and $\beta$. It holds that

$$
\begin{aligned}
\left|Z_{B}(s)\right|^{2} & \leq|z|^{2}+\int_{s}^{t} \frac{\mathrm{d}}{\mathrm{d} \tau}\left|Z_{B}(\tau)\right|^{2} \mathrm{~d} \tau \leq R^{2}+\int_{s}^{t}\left|X_{B}(\tau)\right|\left|V_{B}(\tau)\right|+\left|V_{B}(\tau)\right|\left\|\partial_{x} \psi_{f_{B}}(\tau)\right\|_{\infty} \mathrm{d} \tau \\
& \leq R^{2}+\int_{s}^{T}\left|Z_{B}(\tau)\right|^{2} \mathrm{~d} \tau+\int_{s}^{T}\left|Z_{B}(\tau)\right|\left\|\partial_{x} \psi_{f_{B}}(\tau)\right\|_{\infty} \mathrm{d} \tau .
\end{aligned}
$$

Hence by Gronwall's lemma,

$$
\left|Z_{B}(s)\right|^{2} \leq C+C \int_{s}^{T}\left|Z_{B}(\tau)\right|\left\|\partial_{x} \psi_{f_{B}}(\tau)\right\|_{\infty} \mathrm{d} \tau
$$

Now applying the quadratic version of Gronwall's lemma yields

$$
\left\|Z_{B}(s)\right\|_{L^{\infty}\left(B_{R}(0)\right)} \leq C+C \int_{s}^{T}\left\|\partial_{x} \psi_{f_{B}}(\tau)\right\|_{\infty} \mathrm{d} \tau \leq C=: R_{Z} .
$$

For any $\tau \in[0, T]$,

$$
\begin{aligned}
\left|\partial_{z_{i}} \dot{Z}_{B}(\tau)\right| & \leq\left|\partial_{z_{i}} V_{B}(\tau)\right|+\left|\partial_{z_{i}}\left(\partial_{x} \psi_{f_{B}}\left(\tau, X_{B}(\tau)\right)\right)\right|+\left|\partial_{z_{i}}\left(V_{B}(\tau) \times B\left(\tau, X_{B}(\tau)\right)\right)\right| \\
& \leq C\left(1+\left\|D_{x}^{2} \psi_{f_{B}}(\tau)\right\|_{\infty}+\|B(\tau)\|_{\infty}+\left\|D_{x} B(\tau)\right\|_{\infty}\right)\left|\partial_{z_{i}} Z_{B}(\tau)\right|
\end{aligned}
$$

Hence

$$
\left|\partial_{z_{i}} Z_{B}(s)\right| \leq 1+\int_{s}^{t} C\left(1+\left\|D_{x}^{2} \psi_{f_{B}}(\tau)\right\|_{\infty}+\|B(\tau)\|_{W^{1, \infty}}\right)\left|\partial_{z_{i}} Z_{B}(\tau)\right| \mathrm{d} \tau
$$

and then Gronwall's Lemma implies that

$$
\left\|D_{z} Z_{B}(s)\right\|_{L^{\infty}\left(B_{R}(0)\right)} \leq \exp \left(C \int_{s}^{t}\left(1+\left\|D_{x}^{2} \psi_{f_{B}}(\tau)\right\|_{\infty}+\|B(\tau)\|_{W^{1, \infty}}\right) \mathrm{d} \tau\right)
$$




$$
\leq C \exp \left(C \int_{s}^{t}\left\|D_{x}^{2} \psi_{f_{B}}(\tau)\right\|_{\infty} \mathrm{d} \tau\right)
$$

An estimate to bound $\left\|D_{x}^{2} \psi_{f_{B}}(\tau)\right\|_{\infty}$ is presented by G. Rein in [12, page 389]. It states that

$$
\begin{aligned}
& \left\|D_{x}^{2} \psi_{f_{B}}(t)\right\|_{\infty} \leq C\left[\left(1+\left\|\rho_{f_{B}}(t)\right\|_{\infty}\right)\left(1+\ln _{+}\left\|\partial_{x} \rho_{f_{B}}(t)\right\|_{\infty}\right)+\left\|\rho_{f_{B}}(t)\right\|_{L^{1}}\right]
\end{aligned}
$$

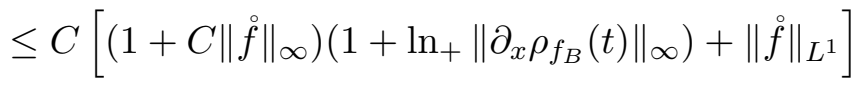

$$
\begin{aligned}
& \leq C+C \ln _{+}\left\|\partial_{x} \rho_{f_{B}}(t)\right\|_{\infty} .
\end{aligned}
$$

Moreover

$$
\begin{aligned}
& \left|\partial_{x} \rho_{f_{B}}(t, x)\right|=\left|\partial_{x} \int_{|v| \leq R} f_{B}(t, x, v) \mathrm{d} v\right| \leq \int_{|v| \leq R}\left|\partial_{x} f(t, x, v)\right| \mathrm{d} v \\
& \leq \frac{4 \pi}{3} R^{3}\left\|\partial_{z} \stackrel{\circ}{f}\right\|_{\infty}\left\|\partial_{z} Z_{B}(0)\right\|_{L^{\infty}\left(B_{R}(0)\right)} \leq \frac{4 \pi}{3} R^{3}\left\|\partial_{z} \stackrel{\circ}{f}\right\|_{\infty} C \exp \left(C \int_{0}^{t}\left\|\partial_{x}^{2} \psi_{f_{B}}(\tau)\right\|_{\infty} \mathrm{d} \tau\right)
\end{aligned}
$$

and now (A.2) implies that

$$
\left\|D_{x}^{2} \psi_{f_{B}}(t)\right\|_{\infty} \leq C+C \int_{0}^{t}\left\|D_{x}^{2} \psi_{f_{B}}(\tau)\right\|_{\infty} \mathrm{d} \tau .
$$

Gronwall's Lemma provides

$$
\left\|D_{x}^{2} \psi_{f_{B}}(t)\right\|_{\infty} \leq C
$$

and since $t$ was arbitrary, this means

$$
\left\|D_{x}^{2} \psi_{f_{B}}(\tau)\right\|_{\infty} \leq C=: c_{3}, \quad \tau \in[0, T] .
$$

Hence, by (A.1),

$$
\left\|D_{z} Z_{B}(s)\right\|_{L^{\infty}\left(B_{R}(0)\right)} \leq C=: c_{1}
$$

and thus

$$
\left\|\partial_{z} f_{B}(t)\right\|_{\infty} \leq\left\|\partial_{z} \stackrel{\circ}{f}\right\|_{\infty}\left\|D_{z} Z_{B}(0)\right\|_{L^{\infty}\left(B_{R}(0)\right)} \leq C=: c_{2} .
$$

Then it finally holds that

$$
\begin{aligned}
& \int_{0}^{T}\left\|\partial_{t} f_{B}(t)\right\|_{\infty}^{2} \mathrm{~d} t=\int_{0}^{T}\left\|v \cdot \partial_{x} f_{B}(t)-\partial_{x} \psi_{f_{B}}(t) \cdot \partial_{v} f_{B}(t)+v \times B(t) \cdot \partial_{v} f(t)\right\|_{L^{\infty}\left(B_{R}(0)\right)}^{2} \mathrm{~d} t \\
& \quad \leq C \int_{0}^{T} R^{2}\left\|\partial_{x} f_{B}(t)\right\|_{\infty}^{2}+C\left\|f_{B}(t)\right\|_{\infty}^{2}\left\|\partial_{v} f_{B}(t)\right\|_{\infty}^{2}+R^{2}\|B(t)\|_{\infty}^{2}\left\|\partial_{v} f_{B}(t)\right\|_{\infty}^{2} \mathrm{~d} t \\
& \quad \leq C=: c_{4} .
\end{aligned}
$$


We will now define $F:=-\partial_{x} \psi_{f_{B}}$, i.e., $F_{i}=-\psi_{\partial_{x_{i}} f_{B}}$ for $i=1,2,3$. Recall that $\partial_{x_{k}} f_{B} \in C\left([0, T] \times \mathbb{R}^{6}\right)$ with compact support supp $\partial_{x_{k}} f_{B}(t) \subset B_{R}(0), t \in[0, T]$. From the Hardy-Littlewood-Sobolev inequality (cf. E. Stein [14, p.119]) and (A.3) we can deduce that

$$
\begin{gathered}
\|F(t)\|_{L^{\beta}\left(\mathbb{R}^{3}\right)} \leq C\left\|\partial_{x} f_{B}(t)\right\|_{L^{3 \beta /(2 \beta+3)}} \leq C\left\|\partial_{x} f_{B}(t)\right\|_{L^{\infty}} \leq C, \\
\|D F(t)\|_{L^{\beta}\left(\mathbb{R}^{3}\right)} \leq C\left\|\partial_{x} f_{B}(t)\right\|_{L^{3 \beta /(\beta+3)}} \leq C\left\|\partial_{x} f_{B}(t)\right\|_{L^{\infty}} \leq C .
\end{gathered}
$$

Furthermore the Calderon-Zygmund inequality (Lemma 7) and (A.3) imply that

$$
\left\|D^{2} F(t)\right\|_{L^{\beta}\left(\mathbb{R}^{3}\right)} \leq C\left\|\partial_{x} f_{B}(t)\right\|_{L^{\beta}} \leq C\left\|\partial_{x} f_{B}(t)\right\|_{L^{\infty}} \leq C
$$

and thus $F \in L^{\infty}\left([0, T] ; W^{2, \beta}\left(\mathbb{R}^{3} ; \mathbb{R}^{3}\right)\right) \subset L^{2}\left(0, T ; W^{2, \beta}\left(\mathbb{R}^{3} ; \mathbb{R}^{3}\right)\right)$. Moreover, because of linearity, for any $s, t \in[0, T]$,

$$
\|F(s)-F(t)\|_{W^{2, \beta}} \leq C\left\|\partial_{x} f_{B}(s)-\partial_{x} f_{B}(t)\right\|_{L^{\infty}} \rightarrow 0 \quad \text { if } s \rightarrow t
$$

and hence $F \in C\left([0, T] ; W^{2, \beta}\left(\mathbb{R}^{3} ; \mathbb{R}^{3}\right)\right)$. Analogously to Lemma $10(\mathrm{~d})$ we can choose $\left(F_{k}\right) \subset C^{\infty}\left([0, T] \times \mathbb{R}^{3} ; \mathbb{R}^{3}\right)$ such that $F_{k} \rightarrow F$ in $L^{2}\left(0, T ; W^{2, \beta}\right)$. Without loss of generality, $\left\|F_{k}\right\|_{L^{2}\left(0, T ; W^{2, \beta}\right)} \leq 2\|F\|_{L^{2}\left(0, T ; W^{2, \beta}\right)}$ for all $k \in \mathbb{N}$. Now, for $k \in \mathbb{N}$ let $Z_{k}=Z_{k}(s, t, z)$ denote the solution of the system

$$
\dot{x}=v, \quad \dot{v}=F_{k}+v \times B
$$

with $Z_{k}(t, t, z)=z$. This means that for all $k \in \mathbb{N}$, the map $t \mapsto Z_{k}(s, t, \cdot)$ lies in $C\left([0, T] ; C_{b}^{2}\right)$ and thus also in $L^{\infty}\left(0, T ; W^{2, \beta}\left(B_{R}(0)\right)\right)$. One can easily show that $t \mapsto Z_{k}(s, t, \cdot)$ converges to $t \mapsto Z_{B}(s, t, \cdot)$ in $L^{\infty}\left(0, T ; L^{\infty}\right)$ (confer the methods that are used in the proof of Lemma 16) and, similar to the approach on page 93 ,

$$
\left\|\left[t \mapsto Z_{k}(s, t, \cdot)\right]\right\|_{L^{\infty}\left(0, T ; W^{1, \beta}\left(B_{R}(0)\right)\right)} \leq\left\|\left[t \mapsto Z_{k}(s, t, \cdot)\right]\right\|_{L^{\infty}\left(0, T ; W^{1, \infty}\left(B_{R}(0)\right)\right)} \leq C
$$

for all $s \in[0, T]$ where $C$ depends only on $\dot{f}, T, K$ and $\beta$ but not on $k$. Now let $i, j \in\{1, \ldots, 6\}$ be arbitrary. Then for all $s, t \in[0, T]$ (without loss of generality $s \leq t$ ),

$$
\begin{aligned}
\int_{B_{R}(0)}\left|\partial_{z_{i}} \partial_{z_{j}} Z_{k}(s, t, z)\right|^{\beta} \mathrm{d} z & =\int_{s}^{t} \frac{\mathrm{d}}{\mathrm{d} \tau} \int_{B_{R}(0)}\left|\partial_{z_{i}} \partial_{z_{j}} Z_{k}(\tau, t, z)\right|^{\beta} \mathrm{d} z \mathrm{~d} \tau \\
& =\beta \int_{s}^{t} \int_{B_{R}(0)}\left|\partial_{z_{i}} \partial_{z_{j}} Z_{k}(\tau, t, z)\right|^{\beta-1}\left|\partial_{z_{i}} \partial_{z_{j}} \dot{Z}_{k}(\tau, t, z)\right| \mathrm{d} z \mathrm{~d} \tau
\end{aligned}
$$

where, for all $s, t \in[0, T]$ and $z \in B_{R}(0)$,

$$
\begin{aligned}
\mid \partial_{z_{i}} \partial_{z_{j}} & \dot{Z}_{k}(\tau, t, z) \mid \\
\leq & C\left|\partial_{z_{i}} \partial_{z_{j}} Z_{k}(\tau)\right|\left(1+\left|D_{x} F_{k}\left(\tau, X_{k}(\tau)\right)\right|+\left|B\left(\tau, X_{k}(\tau)\right)\right|+\left|D_{x} B\left(\tau, X_{k}(\tau)\right)\right|\right) \\
& +C\left(\left|D_{x}^{2} F_{k}\left(\tau, X_{k}(\tau)\right)\right|+\left|D_{x} B\left(\tau, X_{k}(\tau)\right)\right|+\left|D_{x}^{2} B\left(\tau, X_{k}(\tau)\right)\right|\right) \\
\leq & C\left|\partial_{z_{i}} \partial_{z_{j}} Z_{k}(\tau)\right|\left(1+\left\|F_{k}(\tau)\right\|_{W^{2, \beta}}+\|B(\tau)\|_{W^{2, \beta}}\right) \\
& +C\left(\left|D_{x}^{2} F_{k}\left(\tau, X_{k}(\tau)\right)\right|+\left|D_{x} B\left(\tau, X_{k}(\tau)\right)\right|+\left|D_{x}^{2} B\left(\tau, X_{k}(\tau)\right)\right|\right) .
\end{aligned}
$$


Thus, applying Hölder's inequality with exponents $p=\frac{\beta}{\beta-1}$ and $q=\beta$,

$$
\begin{aligned}
& \int_{B_{R}(0)}\left|\partial_{z_{i}} \partial_{z_{j}} Z_{k}(s, t, z)\right|^{\beta} \mathrm{d} z \\
& \leq C \int_{s}^{t} \int_{B_{R}(0)}\left|\partial_{z_{i}} \partial_{z_{j}} Z_{k}(\tau)\right|^{\beta}\left(1+\left\|F_{k}(\tau)\right\|_{W^{2, \beta}}+\|B(\tau)\|_{W^{2, \beta}}\right) \mathrm{d} z \mathrm{~d} \tau \\
& +C \int_{s}^{t} \int_{B_{R}(0)}\left|\partial_{z_{i}} \partial_{z_{j}} Z_{k}(\tau)\right|^{\beta-1}\left(\left|D_{x}^{2} F_{k}\left(\tau, X_{k}\right)\right|+\left|D_{x} B\left(\tau, X_{k}\right)\right|+\left|D_{x}^{2} B\left(\tau, X_{k}\right)\right|\right) \mathrm{d} z \mathrm{~d} \tau \\
& \leq C \int_{s}^{t} \int_{B_{R}(0)}\left|\partial_{z_{i}} \partial_{z_{j}} Z_{k}(\tau)\right|^{\beta} \mathrm{d} z\left(1+\left\|F_{k}(\tau)\right\|_{W^{2, \beta}}+\|B(\tau)\|_{W^{2, \beta}}\right) \mathrm{d} \tau \\
& +C \int_{s}^{t}\left(\int_{B_{R}(0)}\left|\partial_{z_{i}} \partial_{z_{j}} Z_{k}(\tau)\right|^{\beta} \mathrm{d} z\right)^{\frac{\beta-1}{\beta}}\left(\left\|F_{k}(\tau)\right\|_{W^{2, \beta}}+\|B(\tau)\|_{W^{2, \beta}}\right) \mathrm{d} \tau
\end{aligned}
$$

Now we can use the $p$-th power version of Gronwall's lemma with $\left.p=\frac{\beta-1}{\beta} \in\right] 0,1[$ to obtain that

$$
\int_{B_{R}(0)}\left|\partial_{z_{i}} \partial_{z_{j}} Z_{k}(s, t, z)\right|^{\beta} \mathrm{d} z \leq C .
$$

This finally implies that

$$
\left\|\left[t \mapsto Z_{k}(s, t, \cdot)\right]\right\|_{L^{\infty}\left(0, T ; W^{2, \beta}\left(B_{R}(0)\right)\right)} \leq C, \quad s \in[0, T]
$$

where the constant $C$ depends only on $\stackrel{\circ}{f}, T, K$ and $\beta$ but not on $k$. Consequently, due to the Banach-Alaoglu theorem, for any $s \in[0, T]$ there exists some function $\bar{Z}_{s} \in L^{\infty}\left(0, T ; W^{2, \beta}\left(B_{R}(0)\right)\right)$ such that

$$
\left[t \mapsto Z_{k}(s, t, \cdot)\right] \stackrel{*}{\rightarrow} \bar{Z}_{s} \quad \text { in } \quad L^{\infty}\left(0, T ; W^{2, \beta}\left(B_{R}(0)\right)\right)
$$

i.e. for any $\alpha \leq 2$ the map $t \mapsto D_{z}^{\alpha} Z_{k}(s, t, \cdot)$ converges to $D_{z}^{\alpha} \bar{Z}_{s}$ with respect to the weak-*-topology on $\left[L^{1}\left(0, T ; L^{\beta^{\prime}}\right)\right]^{*} \widehat{=} L^{\infty}\left(0, T ; L^{\beta}\right)$ where $1 / \beta+1 / \beta^{\prime}=1$. Because of uniqueness this implies that $\left[t \mapsto Z_{B}(s, t, \cdot)\right]=\bar{Z}_{s} \in L^{\infty}\left(0, T ; W^{2, \beta}\left(B_{R}(0)\right)\right)$ for all $s \in[0, T]$ with

$$
\left\|\left[t \mapsto Z_{B}(s, t, \cdot)\right]\right\|_{L^{\infty}\left(0, T ; W^{2, \beta}\left(B_{R}(0)\right)\right)} \leq C=: c_{5}
$$

From this result we can conclude that $f_{B}$ is twice weakly differentiable with respect to $z$ by chain rule with

$$
\begin{aligned}
& \left\|\partial_{z_{i}} \partial_{z_{j}} f\right\|_{L^{\infty}\left(0, T ; L^{\beta}\right)}=\left\|\partial_{z_{i}} \partial_{z_{j}} f\right\|_{L^{\infty}\left(0, T ; L^{\beta}\left(B_{R}(0)\right)\right)} \\
& \quad \leq\|\stackrel{\circ}{ }\|_{C_{b}^{2}}\|[t \mapsto Z(0, t, \cdot)]\|_{L^{\infty}\left(0, T ; W^{2, \beta}\left(B_{R}(0)\right)\right)} \leq C=: c_{6} .
\end{aligned}
$$

The proof is complete. 
Proof of Lemma 16 Let $B, H \in \mathbb{M}, s, t \in[0, T]$ and $z \in B_{R}(0)$ be arbitrary. Without loss of generality $s \leq t$. Moreover, let $C>0$ denote a generic constant depending only on $f, T$ and $K$ and let $Z_{B}, Z_{H}$ be the solutions of the characteristic system satisfying $Z_{B}(t, t, z)=z$ and $Z_{H}(t, t, z)=z$. We have

$$
\begin{aligned}
& \left|Z_{B}(s)-Z_{H}(s)\right| \leq \int_{s}^{t}\left|\dot{Z}_{B}(\tau)-\dot{Z}_{H}(\tau)\right| \mathrm{d} \tau \\
& \leq \int_{s}^{t}\left|V_{B}(\tau)-V_{H}(\tau)\right| \mathrm{d} \tau+\int_{s}^{t}\left|\partial_{x} \psi_{f_{B}}\left(\tau, X_{B}(\tau)\right)-\partial_{x} \psi_{f_{H}}\left(\tau, X_{H}(\tau)\right)\right| \mathrm{d} \tau \\
& +\int_{s}^{t}\left|V_{B}(\tau) \times B\left(\tau, X_{B}(\tau)\right)-V_{H}(\tau) \times H\left(\tau, X_{H}(\tau)\right)\right| \mathrm{d} \tau \\
& \leq \int_{s}^{t}\left|V_{B}(\tau)-V_{H}(\tau)\right| \mathrm{d} \tau+\int_{s}^{t}\left|\partial_{x} \psi_{f_{B}}\left(\tau, X_{B}(\tau)\right)-\partial_{x} \psi_{f_{B}}\left(\tau, X_{H}(\tau)\right)\right| \mathrm{d} \tau \\
& +\int_{s}^{t}\left|\partial_{x} \psi_{f_{B}}\left(\tau, X_{H}(\tau)\right)-\partial_{x} \psi_{f_{H}}\left(\tau, X_{H}(\tau)\right)\right| \mathrm{d} \tau+\int_{s}^{t}\left|V_{B}(\tau)-V_{H}(\tau)\right|\left|B\left(\tau, X_{B}(\tau)\right)\right| \mathrm{d} \tau \\
& +\int_{s}^{t}\left|V_{H}(\tau)\right|\left|B\left(\tau, X_{B}\right)-B\left(\tau, X_{H}\right)\right| \mathrm{d} \tau+\int_{s}^{t}\left|V_{H}(\tau)\right|\left|B\left(\tau, X_{H}\right)-H\left(\tau, X_{H}\right)\right| \mathrm{d} \tau \\
& \underset{L e m .15}{\leq} \int_{s}^{t} C\left(1+\left\|D_{x}^{2} \psi_{f_{B}}(\tau)\right\|_{\infty}+\|B(\tau)\|_{W^{1, \infty}}\right)\left|Z_{B}(\tau)-Z_{H}(\tau)\right| \mathrm{d} \tau \\
& +\int_{s}^{t}\left\|\partial_{x} \psi_{f_{B}}(\tau)-\partial_{x} \psi_{f_{H}}(\tau)\right\|_{L^{\infty}\left(B_{R_{Z}}(0)\right)} \mathrm{d} \tau+C \int_{s}^{t}\|B(\tau)-H(\tau)\|_{L^{\infty}\left(B_{R_{Z}}(0)\right)} \mathrm{d} \tau \\
& \underset{\substack{\text { Prop. } 8 d \\
\text { Lem. 10b } \\
\text { Lem. } 15}}{\leq} \int_{s}^{t} C\left(1+\|B(\tau)\|_{W^{1, \infty}}\right)\left|Z_{B}(\tau)-Z_{H}(\tau)\right| \mathrm{d} \tau \\
& +C \int_{s}^{t}\left\|f_{B}(\tau)-f_{H}(\tau)\right\|_{\infty} \mathrm{d} \tau+C\|B-H\|_{L^{2}\left(0, T ; W^{1, \beta}\left(B_{R_{Z}}(0)\right)\right)} .
\end{aligned}
$$

Thus by Gronwall's lemma and Lemma 10,

$$
\left|Z_{B}(s)-Z_{H}(s)\right| \leq C \int_{s}^{t}\left\|f_{B}(\tau)-f_{H}(\tau)\right\|_{\infty} \mathrm{d} \tau+C\|B-H\|_{L^{2}\left(0, T ; W^{1, \beta}\left(B_{R_{Z}}(0)\right)\right)}
$$

Now

$$
\begin{aligned}
\left\|f_{B}(t)-f_{H}(t)\right\|_{\infty} & \leq\|D \stackrel{\circ}{f}\|_{\infty}\left\|Z_{B}(0, t, \cdot)-Z_{H}(0, t, \cdot)\right\|_{L^{\infty}\left(B_{R}(0)\right)} \\
& \leq C \int_{0}^{t}\left\|f_{B}(\tau)-f_{H}(\tau)\right\|_{\infty} \mathrm{d} \tau+C\|B-H\|_{L^{2}\left(0, T ; W^{1, \beta}\left(B_{R_{Z}}(0)\right)\right)}
\end{aligned}
$$


which implies that

$$
\left\|f_{B}-f_{H}\right\|_{C\left([0, T] ; C_{b}\right)} \leq L_{1}\|B-H\|_{L^{2}\left(0, T ; W^{1, \beta}\left(B_{R_{Z}}(0)\right)\right)}
$$

if $L_{1}$ is chosen appropriately. Additionally, this yields

$$
\left\|Z_{B}-Z_{H}\right\|_{C\left([0, T] ; C_{b}\left(B_{R}(0)\right)\right)} \leq \ell_{1}\|B-H\|_{L^{2}\left(0, T ; W^{1, \beta}\left(B_{R_{Z}}(0)\right)\right)},
$$

if $\ell_{1}$ is chosen suitably. Hence, according to Proposition 8 (e) and Lemma 15,

$$
\begin{aligned}
& \left|\partial_{x_{i}} \partial_{x_{j}} \psi_{f_{B}}\left(\tau, X_{B}(\tau)\right)-\partial_{x_{i}} \partial_{x_{j}} \psi_{f_{B}}\left(\tau, X_{H}(\tau)\right)\right| \\
& \quad=\left|\partial_{x_{i}} \psi_{\partial_{x_{j}} f_{B}}\left(\tau, X_{B}(\tau)\right)-\partial_{x_{i}} \psi_{\partial_{x_{j}} f_{B}}\left(\tau, X_{H}(\tau)\right)\right| \\
& \quad \leq C\left|X_{B}(\tau)-X_{H}(\tau)\right|^{\gamma} \\
& \quad \leq C\|B-H\|_{L^{2}\left(0, T ; W^{1, \beta}\left(B_{R_{Z}}(0)\right)\right)}^{\gamma}
\end{aligned}
$$

for every $\tau \in[0, T]$ and every $i, j \in\{1, \ldots, 6\}$.

Let now $i \in\{1, \ldots, 6\}$ be arbitrary. It holds that

$$
\begin{aligned}
& \left|\partial_{z_{i}} Z_{B}(s)-\partial_{z_{i}} Z_{H}(s)\right| \\
& \leq \int_{s}^{t}\left|\partial_{z_{i}} \dot{Z}_{B}(\tau)-\partial_{z_{i}} \dot{Z}_{H}(\tau)\right| \mathrm{d} \tau \\
& \leq \int_{s}^{t}\left|\partial_{z_{i}} V_{B}(\tau)-\partial_{z_{i}} V_{H}(\tau)\right| \mathrm{d} \tau \\
& +\int_{s}^{t}\left|D_{x}^{2} \psi_{f_{B}}\left(\tau, X_{B}\right) \partial_{z_{i}} X_{B}-D_{x}^{2} \psi_{f_{H}}\left(\tau, X_{H}\right) \partial_{z_{i}} X_{H}(\tau)\right| \mathrm{d} \tau \\
& +\int_{s}^{t}\left|\partial_{z_{i}} V_{B} \times B\left(\tau, X_{B}\right)-\partial_{z_{i}} V_{H} \times H\left(\tau, X_{H}\right)\right| \mathrm{d} \tau \\
& +\int_{s}^{t}\left|V_{B} \times D_{x} B\left(\tau, X_{B}\right) \partial_{z_{i}} X_{B}-V_{H} \times D_{x} H\left(\tau, X_{H}\right) \partial_{z_{i}} X_{H}\right| \mathrm{d} \tau \\
& \underset{\text { Lem. } 15}{\leq} C \int_{s}^{t}\left(1+\left\|D_{x}^{2} \psi_{f_{B}}(\tau)\right\|_{\infty}+\|B(\tau)\|_{W^{1, \infty}}\right)\left|\partial_{z_{i}} Z_{B}(\tau)-\partial_{z_{i}} Z_{H}(\tau)\right| \mathrm{d} \tau \\
& +C \int_{s}^{t}\left|D_{x}^{2} \psi_{f_{B}}\left(\tau, X_{B}\right)-D_{x}^{2} \psi_{f_{H}}\left(\tau, X_{H}\right)\right| \mathrm{d} \tau+C \int_{s}^{t}\left|B\left(\tau, X_{B}\right)-H\left(\tau, X_{H}\right)\right| \mathrm{d} \tau \\
& +C \int_{s}^{t}\left|D_{x} B\left(\tau, X_{B}\right)-D_{x} H\left(\tau, X_{H}\right)\right| \mathrm{d} \tau+C \int_{s}^{t}\left\|D_{x} B(\tau)\right\|_{\infty}\left|V_{B}-V_{H}\right| \mathrm{d} \tau
\end{aligned}
$$




$$
\begin{aligned}
& \underset{\substack{\text { Prop. } 8 d, \\
\text { Lem.15 }}}{\leq} C \int_{s}^{t}\left(1+\|B(\tau)\|_{W^{1, \infty}}\right)\left|\partial_{z_{i}} Z_{B}(\tau)-\partial_{z_{i}} Z_{H}(\tau)\right| \mathrm{d} \tau \\
& \text { Eem. } 15 \\
& +C \int_{s}^{t}\left|X_{B}(\tau)-X_{H}(\tau)\right|^{\gamma} \mathrm{d} \tau+C \int_{s}^{t}\left\|\partial_{z} f_{B}(\tau)-\partial_{z} f_{H}(\tau)\right\|_{L^{\infty}\left(B_{R_{Z}}(0)\right)} \mathrm{d} \tau \\
& +C \int_{s}^{t}\left\|D_{x} B(\tau)\right\|_{\infty}\left|X_{B}(\tau)-X_{H}(\tau)\right| \mathrm{d} \tau+C \int_{s}^{t}\|B(\tau)-H(\tau)\|_{\infty} \mathrm{d} \tau \\
& +C\left\|X_{B}-X_{H}\right\|_{\infty}^{\gamma} \int_{0}^{T}\left[D_{x} B(\tau)\right]_{\gamma} \mathrm{d} \tau+C \int_{s}^{t}\left\|D_{x} B(\tau)\right\|_{\infty}\left|V_{B}-V_{H}\right| \mathrm{d} \tau \\
& +C \int_{s}^{t}\left\|D_{x} B(\tau)-D_{x} H(\tau)\right\|_{L^{\infty}\left(B_{R_{Z}}(0)\right)} \mathrm{d} \tau \\
& \underset{\substack{\text { Lem. 10 } \\
\text { Eq. A.5) }}}{\leq} C \int_{s}^{t}\left(1+\|B(\tau)\|_{C^{1, \gamma}}\right)\left|\partial_{z_{i}} Z_{B}(\tau)-\partial_{z_{i}} Z_{H}(\tau)\right| \mathrm{d} \tau \\
& +C \int_{s}^{t}\left\|\partial_{z} f_{B}(\tau)-\partial_{z} f_{H}(\tau)\right\|_{\infty} \mathrm{d} \tau+C\|B-H\|_{L^{2}\left(0, T ; W^{2, \beta}\left(B_{R_{Z}}(0)\right)\right)}^{\gamma} \\
& +C \int_{s}^{t}\|B(\tau)-H(\tau)\|_{W^{2, \beta}\left(B_{R_{Z}}(0)\right)} \mathrm{d} \tau \\
& \underset{\text { Lem.10 }}{\leq} C \int_{s}^{t}\left(1+\|B(\tau)\|_{W^{2, \beta}}\right)\left|\partial_{z_{i}} Z_{B}(\tau)-\partial_{z_{i}} Z_{H}(\tau)\right| \mathrm{d} \tau \\
& +C \sqrt{T} K^{(1-\gamma) / 2}\left(\int_{0}^{T}\|B(\tau)-H(\tau)\|_{W^{2, \beta}\left(B_{R_{Z}}(0)\right)}^{2} \mathrm{~d} \tau\right)^{\frac{\gamma}{2}} \\
& +C \int_{s}^{t}\left\|\partial_{z} f_{B}(\tau)-\partial_{z} f_{H}(\tau)\right\|_{\infty} \mathrm{d} \tau+C\|B-H\|_{L^{2}\left(0, T ; W^{2, \beta}\left(B_{R_{Z}}(0)\right)\right)}^{\gamma} \\
& \leq C \int_{s}^{t}\left(1+\|B(\tau)\|_{W^{2, \beta}}\right)\left|\partial_{z_{i}} Z_{B}(\tau)-\partial_{z_{i}} Z_{H}(\tau)\right| \mathrm{d} \tau \\
& +C \int_{s}^{t}\left\|\partial_{z} f_{B}(\tau)-\partial_{z} f_{H}(\tau)\right\|_{\infty} \mathrm{d} \tau+C\|B-H\|_{L^{2}\left(0, T ; W^{2, \beta}\left(B_{R_{Z}}(0)\right)\right)}^{\gamma} .
\end{aligned}
$$


Again, Gronwall's lemma implies that

$$
\begin{aligned}
& \left|\partial_{z_{i}} Z_{B}(s)-\partial_{z_{i}} Z_{H}(s)\right| \\
& \leq\left(C\|B-H\|_{L^{2}\left(0, T ; W^{2, \beta}\left(B_{R_{Z}}(0)\right)\right.}^{\gamma}+C \int_{s}^{t}\left\|\partial_{z} f_{B}(\tau)-\partial_{z} f_{H}(\tau)\right\|_{\infty}\right) \\
& \quad \cdot \exp \left(\int_{0}^{T} 1+\|B(\tau)\|_{W^{2, \beta}} \mathrm{d} \tau\right) \\
& \leq C\|B-H\|_{L^{2}\left(0, T ; W^{2, \beta}\left(B_{R_{Z}}(0)\right)\right)}^{\gamma}+C \int_{s}^{t}\left\|\partial_{z} f_{B}(\tau)-\partial_{z} f_{H}(\tau)\right\|_{\infty} .
\end{aligned}
$$

Finally

$$
\begin{aligned}
& \left\|\partial_{z} f_{B}(t)-\partial_{z} f_{H}(t)\right\|_{\infty}=\left\|\partial_{z} f_{B}(t)-\partial_{z} f_{H}(t)\right\|_{L^{\infty}\left(B_{R}(0)\right)} \\
& \quad \leq C\left\|\partial_{z} Z_{B}(0)-\partial_{z} Z_{H}(0)\right\|_{L^{\infty}\left(B_{R}(0)\right)}+C\left\|Z_{B}(0)-Z_{H}(0)\right\|_{L^{\infty}\left(B_{R}(0)\right)} \\
& \quad \leq \quad C\|B-H\|_{L^{2}\left(0, T ; W^{2, \beta}\left(B_{R_{Z}}(0)\right)\right)}^{\gamma}+C \int_{0}^{t}\left\|\partial_{z} f_{B}(\tau)-\partial_{z} f_{H}(\tau)\right\|_{\infty} \\
& \leq \underset{E q . \text { (A.5), (A.9) }}{\leq}
\end{aligned}
$$

and hence

$$
\begin{aligned}
\left\|\partial_{z} f_{B}-\partial_{z} f_{H}\right\|_{C\left([0, T] ; C_{b}\right)} & \leq L_{2}\|B-H\|_{L^{2}\left(0, T ; W^{2, \beta}\left(B_{R_{Z}}(0)\right)\right)}^{\gamma} \\
\left\|\partial_{z_{i}} Z_{B}-\partial_{z_{i}} Z_{H}\right\|_{C\left([0, T] ; C_{b}\left(B_{R}(0)\right)\right)} & \leq \ell_{2}\|B-H\|_{L^{2}\left(0, T ; W^{2, \beta}\left(B_{R_{Z}}(0)\right)\right)}^{\gamma}
\end{aligned}
$$

if $\ell_{2}$ and $L_{2}$ are chosen appropriately. The third assertion can simply be proved by

$$
\begin{aligned}
& \int_{0}^{T}\left\|\partial_{t} f_{B}(t)-\partial_{t} f_{H}(t)\right\|_{\infty}^{2} \mathrm{~d} t \\
& \begin{aligned}
= & \int_{0}^{T} \| v \cdot \partial_{x}\left(f_{B}(t)-f_{H}(t)\right)-\partial_{x} \psi_{f_{B}}(t) \cdot \partial_{v}\left(f_{B}(t)-f_{H}(t)\right) \\
& -\partial_{x} \psi_{f_{B}-f_{H}}(t) \cdot \partial_{v} f_{H}(t)+(v \times B(t)) \cdot \partial_{v}\left(f_{B}(t)-f_{H}(t)\right)
\end{aligned} \\
& +(v \times(B(t)-H(t))) \cdot \partial_{v} f_{H}(t) \|_{L^{\infty}\left(B_{R}(0)\right)}^{2} \mathrm{~d} t \\
& \leq C \int_{0}^{T}\left\|\partial_{z} f_{B}(t)-\partial_{z} f_{H}(t)\right\|_{\infty}^{2} \mathrm{~d} t+C \int_{0}^{T}\left\|\partial_{x} \psi_{f_{B}}(t)-\partial_{x} \psi_{f_{H}}(t)\right\|_{\infty}^{2} \mathrm{~d} t \\
& +C \int_{0}^{T}\|B(t)-H(t)\|_{L^{\infty}\left(B_{R}(0)\right)}^{2} \mathrm{~d} t \\
& \underset{\substack{\text { Prop.8d } \\
\text { Eq.(A.4),(A.8) }}}{\leq} C \int_{0}^{T}\|B(t)-H(t)\|_{\left.W^{2, \beta}\left(B_{R_{Z}}(0)\right)\right)}^{2 \gamma} \mathrm{d} t+C \int_{0}^{T}\|B(t)-H(t)\|_{\left.W^{2, \beta}\left(B_{R_{Z}}(0)\right)\right)}^{2} \mathrm{~d} t \\
& \leq L_{3}^{2}\|B-H\|_{L^{2}\left(0, T ; W^{2, \beta}\left(B_{R_{Z}}(0)\right)\right)}^{2 \gamma}
\end{aligned}
$$

if $L_{3}$ is chosen suitably. The proof is complete. 
Proof of Proposition 24 Let $c>0$ denote a generic constant depending only on $r_{0}, r_{2}$, $T,\|\mathbf{a}\|_{C\left([0, T] ; C_{b}^{1}\right)},\|\mathbf{b}\|_{C\left([0, T] ; C_{b}^{1}\right)},\|\mathbf{f}\|_{C_{b}^{2}},\|\mathbf{A}\|_{C\left([0, T] ; C^{1, \gamma}\right)},\|\mathbf{B}\|_{C\left([0, T] ; C^{1, \gamma}\right)},\|\mathbf{C}\|_{C\left([0, T] ; C_{b}^{1}\right)}$ and $\|\chi\|_{C_{b}^{1}}$.

For $t \in[0, T]$ and $z \in \mathbb{R}^{6}$ let $Z=(X, V)(s, t, z)$ denote the solution of the characteristic system with $Z(t, t, z)=z$. Moreover, for $t \in[0, T]$ and $z \in \mathbb{R}^{6}$, we define a recursive sequence by

$$
\begin{aligned}
f_{0}(t, z) & :=\stackrel{\circ}{\mathbf{f}}(z) \\
f_{n+1}(t, z) & :=\stackrel{\circ}{\mathbf{f}}(Z(0, t, z))+\int_{0}^{t}\left[\partial_{x} \psi_{f_{n}} \cdot \mathbf{C}+\chi \Phi_{\mathbf{a}, f_{n}}+\mathbf{b}\right](s, Z(s, t, z)) \mathrm{d} s, \quad n \in \mathbb{N}_{0} .
\end{aligned}
$$

By induction we can conclude that all $f_{n}$ are continuous. Then for any fixed $\tau \in[0, T]$ and $n \in \mathbb{N}$ the functions $\stackrel{\mathbf{f}}{\mathbf{f}},\left[\partial_{x} \psi_{f_{n}} \cdot \mathbf{C}\right](\tau),\left[\chi \Phi_{\mathbf{a}, f_{n}}\right](\tau)$ and $\mathbf{b}(\tau)$ are continuous and compactly supported in $B_{r}(0)$ with $r:=\max \left\{r_{0}, r_{2}\right\}$. This directly implies that $f_{0}(t)$ is compactly supported with supp $f_{0}(t) \subset B_{r}(0)$ for all $t \in[0, T]$. Moreover for any $\tau \in[0, T]$,

$$
\left.\begin{array}{rl}
\operatorname{supp} \stackrel{\circ}{\mathbf{f}}(Z(s, t, \cdot)) & =Z(t, s, \operatorname{supp} \stackrel{\circ}{\mathbf{f}}) \\
\operatorname{supp}\left[\partial_{x} \psi_{f} \cdot \mathbf{C}\right](\tau, Z(s, t, \cdot)) & =Z\left(t, s, \operatorname{supp} \partial_{x} \psi_{f_{n}} \cdot \mathbf{C}(\tau)\right) \\
\operatorname{supp}\left[\chi \Phi_{\mathbf{a}, f_{n}}\right](\tau, Z(s, t, \cdot)) & =Z\left(t, s, \operatorname{supp} \chi \Phi_{\mathbf{a}, f_{n}}(\tau)\right) \\
\operatorname{supp} \mathbf{b}(\tau, Z(s, t, \cdot)) & =Z(t, s, \operatorname{supp} \mathbf{b}(\tau))
\end{array}\right\} \underset{\text { Lemma } 23}{\subset} B_{\zeta(r)}(0) \text {. }
$$

If we choose $\tau=s$ we can inductively deduce that $\operatorname{supp} f_{n}(t) \subset B_{\zeta(r)}(0)$ for all $t \in[0, T]$ and all $n \in \mathbb{N}$. Finally, by another induction, $f_{n} \in C^{1}(] 0, T\left[\times \mathbb{R}^{6}\right)$ as the partial derivatives can be recursively described by:

$$
\begin{aligned}
& \partial_{t} f_{0}(t, z)=0 \\
& \partial_{z_{i}} f_{0}(t, z)=\partial_{z_{i}} \stackrel{\circ}{\mathbf{f}}(z) \\
& \partial_{t} f_{n+1}(t, z)=\partial_{z} \stackrel{\circ}{\mathbf{f}}(Z(0, t, z)) \cdot \partial_{t} Z(0, t, z)+\partial_{x} \psi_{f_{n}} \cdot \mathbf{C}(t, z)+\chi \Phi_{\mathbf{a}, f_{n}}(t, z)+\mathbf{b}(t, z) \\
& +\int_{0}^{t} \partial_{z}\left[\partial_{x} \psi_{f_{n}} \cdot \mathbf{C}+\chi \Phi_{\mathbf{a}, f_{n}}+\mathbf{b}\right](s, Z(s, t, z)) \cdot \partial_{t} Z(s, t, z) \mathrm{d} s \\
& \partial_{z_{i}} f_{n+1}(t, z)=\partial_{z} \stackrel{\circ}{\mathbf{f}}(Z(0, t, z)) \cdot \partial_{z_{i}} Z(0, t, z) \\
& +\int_{0}^{t} \partial_{z}\left[\partial_{x} \psi_{f_{n}} \cdot \mathbf{C}+\chi \Phi_{\mathbf{a}, f_{n}}+\mathbf{b}\right](s, Z(s, t, z)) \cdot \partial_{z_{i}} Z(s, t, z) \mathrm{d} s .
\end{aligned}
$$

where

$\partial_{z}\left[\partial_{x} \psi_{f} \cdot \mathbf{C}+\chi \Phi_{\mathbf{a}, f_{n}}+\mathbf{b}\right]=\left(\begin{array}{c}D_{x}^{2} \psi_{f_{n}} \mathbf{C}+D_{x} \mathbf{C} \partial_{x} \psi_{f_{n}}+\partial_{x} \chi \Phi_{\mathbf{a}, f_{n}}+\chi \Phi_{\mathbf{a}, f_{n}}^{\prime}+\partial_{x} \mathbf{b} \\ D_{v} \mathbf{C} \partial_{x} \psi_{f_{n}}+\partial_{v} \chi \Phi_{\mathbf{a}, f_{n}}+\partial_{v} \mathbf{b}\end{array}\right)$ 
Hence one can easily show that

$$
\left\|f_{1}(t)-f_{0}(t)\right\|_{\infty} \leq c,\left\|\partial_{t} f_{1}(t)-\partial_{t} f_{0}(t)\right\|_{\infty} \leq c,\left\|\partial_{z_{i}} f_{1}(t)-\partial_{z_{i}} f_{0}(t)\right\|_{\infty} \leq c .
$$

Furthermore we obtain the following estimates:

$$
\begin{aligned}
& \left\|f_{n+1}(t)-f_{n}(t)\right\|_{\infty} \\
& \leq c \int_{0}^{t}\left\|\partial_{x} \psi_{f_{n}-f_{n-1}}(s, X(s))\right\|_{\infty}+\left\|\Phi_{\mathbf{a}, f_{n}-f_{n-1}}(s, X(s))\right\|_{\infty} \mathrm{d} s \\
& \leq c \int_{0}^{t}\left\|f_{n}(s)-f_{n-1}(s)\right\|_{\infty} \mathrm{d} s, \\
& \left\|\partial_{z_{i}} f_{n+1}(t)-\partial_{z_{i}} f_{n}(t)\right\|_{\infty} \\
& \leq c \int_{0}^{t}\left\|D_{x}^{2} \psi_{f_{n-f_{n-1}}}(s, X(s))\right\|_{\infty}+\left\|\partial_{x} \psi_{f_{n-f_{n-1}}}(s, X(s))\right\|_{\infty} \mathrm{d} s \\
& +c \int_{0}^{t}\left\|\Phi_{\mathbf{a}, f_{n}-f_{n-1}}(s, X(s))\right\|_{\infty}+\left\|\Phi_{\mathbf{a}, f_{n}-f_{n-1}}^{\prime}(s, X(s))\right\|_{\infty} \mathrm{d} s \\
& \leq c \int_{0}^{t}\left\|f_{n}(s)-f_{n-1}(s)\right\|_{W^{1, \infty}} \mathrm{d} s \\
& \left\|\partial_{t} f_{n+1}(t)-\partial_{t} f_{n}(t)\right\|_{\infty} \\
& \leq\left\|\partial_{x} \psi_{f_{n}-f_{n-1}}(t)\right\|_{\infty}+\left\|\Phi_{\mathbf{a}, f_{n}-f_{n-1}}(t)\right\|_{\infty} \\
& +c \int_{0}^{t}\left\|D_{x}^{2} \psi_{f_{n-f_{n-1}}}(s, X(s))\right\|_{\infty}+\left\|\partial_{x} \psi_{f_{n-f_{n-1}}}(s, X(s))\right\|_{\infty} \mathrm{d} s \\
& +c \int_{0}^{t}\left\|\Phi_{\mathbf{a}, f_{n}-f_{n-1}}(s, X(s))\right\|_{\infty}+\left\|\Phi_{\mathbf{a}, f_{n}-f_{n-1}}^{\prime}(s, X(s))\right\|_{\infty} \mathrm{d} s \\
& \leq c\left\|f_{n}(t)-f_{n-1}(t)\right\|_{\infty}+c \int_{0}^{t}\left\|f_{n}(s)-f_{n-1}(s)\right\|_{W^{1, \infty}} \mathrm{d} s \\
& \leq c \int_{0}^{t}\left\|\partial_{t} f_{n}(s)-\partial_{t} f_{n-1}(s)\right\|_{\infty} \mathrm{d} s+c \int_{0}^{t}\left\|f_{n}(s)-f_{n-1}(s)\right\|_{W^{1, \infty}} \mathrm{d} s .
\end{aligned}
$$

Hence there exists some constant $c_{*}>0$ such that for all $t \in[0, T]$,

$$
M_{1,0}(t) \leq c_{*} \quad \text { and } \quad M_{n+1, n}(t) \leq c_{*} \int_{0}^{t} M_{n, n-1}(s) \mathrm{d} s, \quad n \in \mathbb{N}
$$


where

$$
M_{m, n}(t):=\max \left\{\left\|f_{m}(t)-f_{n}(t)\right\|_{\infty},\left\|\partial_{t} f_{m}(t)-\partial_{t} f_{n}(t)\right\|_{\infty},\left\|\partial_{z} f_{m}(t)-\partial_{z} f_{n}(t)\right\|_{\infty}\right\}
$$

for $m, n \in \mathbb{N}_{0}$. Thus by induction,

$$
M_{n+1, n}(t) \leq c_{*} \frac{t^{n}}{n !} \leq c_{*} \frac{T^{n}}{n !}, \quad t \in[0, T], n \in \mathbb{N}
$$

and hence for $m, n \in \mathbb{N}$ with $n<m$,

$$
M_{m, n}(t) \leq \sum_{j=n}^{m-1} M_{j+1, j}(t) \leq \sum_{j=n}^{\infty} c_{*} \frac{T^{j}}{j !} \rightarrow 0, \quad n \rightarrow \infty .
$$

Consequently $\left(f_{n}\right)$ is a Cauchy-sequence in $C_{b}^{1}\left([0, T] \times \mathbb{R}^{6}\right)$ and converges to some function $f \in C_{b}^{1}\left([0, T] \times \mathbb{R}^{6}\right)$ because of completeness. Obviously, as the radius $\zeta(r)$ does not depend on $n$,

$$
\operatorname{supp} f(t) \subset \overline{B_{\zeta(r)}(0)} \subset B_{\zeta(r+1)}(0), \quad t \in[0, T]
$$

and $f$ satisfies the equation

$$
f(t, z)=\stackrel{\circ}{\mathbf{f}}(Z(0, t, z))+\int_{0}^{t}\left[\partial_{x} \psi_{f} \cdot \mathbf{C}+\Phi_{\mathbf{a}, f}+\mathbf{b}\right](s, Z(s, t, z)) \mathrm{d} s .
$$

The function $f$ is a solution of the initial value problem because for every $t \in[0, T]$ and $z \in \mathbb{R}^{6}$ it holds that $f(0, z)=\stackrel{\circ}{\mathbf{f}}(z)$ and

$$
\begin{aligned}
0= & {\left.\left[\frac{\mathrm{d}}{\mathrm{d} \tau} \mathfrak{\mathbf { f }}(Z(0, t, z))\right]\right|_{\tau=t}=\left[\left.\frac{\mathrm{d}}{\mathrm{d} \tau} \stackrel{\circ}{\mathbf{f}}(Z(0, \tau, Z(\tau, t, z))]\right|_{\tau=t}\right.} \\
= & {\left.\left[\frac{\mathrm{d}}{\mathrm{A} .10)} f(\tau, Z(\tau, t, z))-\frac{\mathrm{d}}{\mathrm{d} \tau} \int_{0}^{\tau}\left[\partial_{x} \psi_{f} \cdot \mathbf{C}+\Phi_{\mathbf{a}, f}+\mathbf{b}\right](s, Z(s, \tau, Z(\tau, t, z))) \mathrm{d} s\right]\right|_{\tau=t} } \\
= & {\left.\left[\frac{\mathrm{d}}{\mathrm{d} \tau} f(\tau, Z(\tau, t, z))-\frac{\mathrm{d}}{\mathrm{d} \tau} \int_{0}^{\tau}\left[\partial_{x} \psi_{f} \cdot \mathbf{C}+\Phi_{\mathbf{a}, f}+\mathbf{b}\right](s, Z(s, t, z)) \mathrm{d} s\right]\right|_{\tau=t} } \\
= & {\left[\partial_{t} f(\tau, Z(\tau, t, z))+V(\tau, t, z) \cdot \partial_{x} f(\tau, Z(\tau, t, z))+\mathbf{A}(\tau, X(\tau, t, z)) \cdot \partial_{v} f(\tau, Z(\tau, t, z))\right.} \\
& \quad+(V(\tau, t, z) \times \mathbf{B}(\tau, X(\tau, t, z))) \cdot \partial_{v} f(\tau, Z(\tau, t, z))-\mathbf{b}(\tau, Z(\tau, t, z)) \\
& \left.\quad-\chi(\tau, Z(\tau, t, z)) \Phi_{\mathbf{a}, f}(\tau, X(\tau, t, z))-\partial_{x} \psi_{f}(\tau, X(\tau, t, z)) \cdot \mathbf{C}(\tau, Z(\tau, t, z))\right]\left.\right|_{\tau=t} \\
= & \partial_{t} f(t, z)+v \cdot \partial_{x} f(t, z)+\mathbf{A}(t, x) \cdot \partial_{v} f(t, z)+(v \times \mathbf{B}(t, x)) \cdot \partial_{v} f(t, z) \\
& \quad-\partial_{x} \psi_{f}(t, x) \cdot \mathbf{C}(t, x, v)-\chi(t, x, v) \Phi_{\mathbf{a}, f}(t, x)-b(t, x, v) .
\end{aligned}
$$


We will finally prove uniqueness by assuming that there exists another solution $\tilde{f}$ of the initial value problem and define $d:=f-\tilde{f}$. Then for any $t \in[0, T]$,

$$
\begin{aligned}
\|d(t)\|_{L^{2}}^{2}= & \int d(t)^{2} \mathrm{~d} z=2 \int_{0}^{t} \int \partial_{t} d(s) d(s) \mathrm{d} z \mathrm{~d} s \\
= & 2 \int_{0}^{t} \int-v \cdot \partial_{x} d(s) d(s)-\mathbf{A}(s) \cdot \partial_{v} d(s) d(s)-(v \times \mathbf{B}(s)) \cdot \partial_{v} d(s) d(s) \\
& \quad+\partial_{x} \psi_{d(s)} \cdot \mathbf{C}(s) d(s)+\chi \Phi_{\mathbf{a}, d}(s) d(s) \mathrm{d} z \mathrm{~d} s \\
= & 2 \int_{0}^{t} \int_{x} \partial_{x} \psi_{d(s)} \cdot \mathbf{C}(s) d(s)+\chi \Phi_{\mathbf{a}, d}(s) d(s) \mathrm{d} z \mathrm{~d} s \\
\leq & c \int_{0}^{t}\|d(s)\|_{L^{2}}^{2} \mathrm{~d} s
\end{aligned}
$$

and hence $\|d(t)\|_{L^{2}}=0$ for all $t \in[0, T]$ once again by Gronwall's lemma. This directly implies that $f=\tilde{f}$ almost everywhere which means uniqueness of the solution $f$.

Proof of Corollary 26 In (a) the coefficients satisfy the regularity assumptions (5.10). Because of density we can choose sequences $\left(\mathbf{b}_{k}\right) \subset C\left([0, T] ; C_{b}^{1}\right),\left(\stackrel{\circ}{\mathbf{f}}_{k}\right) \subset C_{c}^{2}\left(\mathbb{R}^{6}\right)$, $\left(\mathbf{B}_{k}\right) \subset C\left([0, T] ; C^{1, \gamma}\right)$ and $\left(\mathbf{C}_{k}\right) \subset C\left([0, T] ; C_{b}^{1}\right)$ such that

$$
\begin{aligned}
& \mathbf{b}_{k} \rightarrow \mathbf{b} \text { in } L^{2}\left(0, T ; C_{b} \cap H^{1}\right), \quad\left\|\mathbf{b}_{k}\right\|_{L^{2}\left(0, T ; H^{1}\right)} \leq 2\|\mathbf{b}\|_{L^{2}\left(0, T ; H^{1}\right)}, \\
& \left\|\mathbf{b}_{k}\right\|_{L^{2}\left(0, T ; C_{b}\right)} \leq 2\|\mathbf{b}\|_{L^{2}\left(0, T ; C_{b}\right)}, \\
& \stackrel{\circ}{\mathbf{f}}_{k} \rightarrow \stackrel{\circ}{\mathbf{f}} \text { in } C_{b}^{1}\left(\mathbb{R}^{6}\right), \quad\left\|\stackrel{\circ}{\mathbf{f}_{k}}\right\|_{C_{b}^{1}} \leq 2\|\stackrel{\circ}{\mathbf{f}}\|_{C_{b}^{1}} \\
& \mathbf{B}_{k} \rightarrow \mathbf{B} \text { in } L^{2}\left(0, T ; C^{1, \gamma}\right), \quad \quad\left\|\mathbf{B}_{k}\right\|_{L^{2}\left(0, T ; C^{1, \gamma}\right)} \leq 2\|\mathbf{B}\|_{L^{2}\left(0, T ; C^{1, \gamma}\right)} \\
& \mathbf{C}_{k} \rightarrow \mathbf{C} \text { in } L^{2}\left(0, T ; H^{1} \cap C_{b}\right), \quad\left\|\mathbf{C}_{k}\right\|_{L^{2}\left(0, T ; H^{1}\right)} \leq 2\|\mathbf{C}\|_{L^{2}\left(0, T ; H^{1}\right)}, \\
& \left\|\mathbf{C}_{k}\right\|_{L^{2}\left(0, T ; C_{b}\right)} \leq 2\|\mathbf{C}\|_{L^{2}\left(0, T ; C_{b}\right)}
\end{aligned}
$$

and for all $t \in[0, T]$,

$$
\operatorname{supp} \mathbf{b}_{k}(t), \operatorname{supp} \stackrel{\circ}{\mathbf{f}}_{k}, \operatorname{supp} \mathbf{C}(t) \subset B_{r_{0}+1}(0) .
$$

Then for every $k \in \mathbb{N}$, according to Proposition 24, there exists a unique classical solution $f_{k}$ of (5.1) to the coefficients $\mathbf{b}_{k}, \stackrel{\circ}{f}_{k}, \mathbf{B}_{k}$ and $\mathbf{C}_{k}$. Moreover for all $t \in[0, T]$,

$$
\operatorname{supp} f_{k}(t) \subset B_{\varrho}(0) \quad \text { with } \varrho:=\zeta\left(2+\max \left\{r_{0}, r_{2}\right\}\right)=\zeta(2+r) \text {. }
$$

Now let $Z_{k}$ denote the solution of the characteristic system to $\mathbf{A}$ and $\mathbf{B}_{k}$ satisfying $Z_{k}(t, t, z)=z$ and let $c>0$ denote some generic constant depending only on $T, r_{0}$,

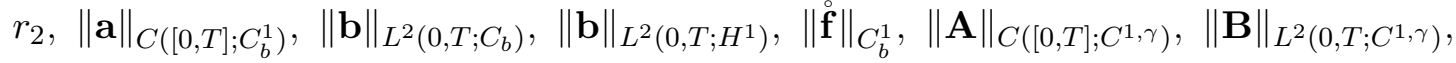
$\|\mathbf{C}\|_{L^{2}\left(0, T ; C_{b} \cap H^{1}\right)}$ and $\|\chi\|_{C_{b}^{1}}$. 
From Lemma 23 we know that for any $r>0$ and all $s, t \in[0, T]$,

$$
\left\|Z_{k}(s, t, \cdot)\right\|_{L^{\infty}\left(B_{r}(0)\right)}<C(r) \quad \text { and } \quad\left\|\partial_{z} Z_{k}(s, t, \cdot)\right\|_{L^{\infty}\left(B_{r}(0)\right)}<C(r)
$$

where $C(r)$ denotes some positive constant depending only on $r,\|\mathbf{A}\|_{L^{2}\left(0, T ; C_{b}^{1}\right)}$ and $\|\mathbf{B}\|_{L^{2}\left(0, T ; C_{b}^{1}\right)}$. Then for any $(t, z) \in[0, T] \times B_{r}(0)$,

$$
\begin{aligned}
& \left|f_{k}(t, z)\right| \leq\left\|\stackrel{\circ}{\mathbf{f}}_{k}\right\|_{\infty}+\int_{0}^{t}\left\|\partial_{x} \psi_{f_{k}}(s)\right\|_{\infty}\left\|\mathbf{C}_{k}(s)\right\|_{\infty}+\left\|\Phi_{\mathbf{a}, f_{k}}(s)\right\|_{\infty}+\left\|\mathbf{b}_{k}(s)\right\|_{\infty} \mathrm{d} s \\
& \leq c+c \int_{0}^{t}\left\|f_{k}(s)\right\|_{\infty}\left(c+\left\|\mathbf{C}_{k}(s)\right\|_{\infty}\right) \mathrm{d} s
\end{aligned}
$$

which yields $\left\|f_{k}(t)\right\|_{L^{\infty}} \leq c$ by Gronwall. The $z$-derivative can be bounded by

$$
\begin{aligned}
& \int_{B_{\varrho}(0)}\left|\partial_{z_{i}} f_{k}(t, z)\right|^{2} \mathrm{~d} z \\
& \leq c\left\|D \mathbf{f}_{k}\right\|_{\infty}^{2} \int_{B_{\varrho}(0)}\left|\partial_{z} Z_{k}(0, t, z)\right|^{2} \mathrm{~d} z \\
& +c \int_{0}^{t} \int_{B_{\varrho}(0)}\left|\left[D_{x}^{2} \psi_{f_{k}} \mathbf{C}_{k}+D_{x} \mathbf{C}_{k} \partial_{x} \psi_{f_{k}}+\partial_{x} \chi \Phi_{\mathbf{a}, f_{k}}+\chi \Phi_{\mathbf{a}, f_{k}}^{\prime}+\partial_{x} \mathbf{b}_{k}\right]\left(s, Z_{k}(s)\right)\right|^{2} \mathrm{~d} s \\
& +c \int_{0}^{t} \int_{B_{\varrho}(0)}\left|\left[D_{v} \mathbf{C}_{k} \partial_{x} \psi_{f_{k}}+\partial_{v} \chi \Phi_{\mathbf{a}, f_{k}}+\partial_{v} \mathbf{b}_{k}\right]\left(s, Z_{k}(s)\right)\right|^{2} \mathrm{~d} s \\
& \leq c+c \int_{0}^{t}\left\|f_{k}(s)\right\|_{L^{\infty}}^{2}\left(\left\|\mathbf{C}_{k}(s)\right\|_{L^{\infty}}^{2}+\left\|\mathbf{C}_{k}(s)\right\|_{H^{1}}^{2}\right)+\left\|\partial_{z} f_{k}(s)\right\|_{L^{2}}^{2}+\left\|\mathbf{b}_{k}(s)\right\|_{H^{1}}^{2} \mathrm{~d} s
\end{aligned}
$$

and hence,

$$
\left\|\partial_{z} f_{k}(t)\right\|_{L^{2}}^{2}=\left\|\partial_{z} f_{k}(t)\right\|_{L^{2}\left(B_{\varrho}(0)\right)}^{2} \leq c+c \int_{0}^{t}\left\|\partial_{z} f_{k}(s)\right\|_{L^{2}}^{2} \mathrm{~d} s
$$

which implies that $\left\|\partial_{z} f_{k}(t)\right\|_{L^{2}} \leq c$ for all $t \in[0, T]$. Finally

$$
\begin{aligned}
& \int_{0}^{T} \int_{B_{\varrho}(0)}\left|\partial_{t} f_{k}(t, z)\right|^{2} \mathrm{~d} z \mathrm{~d} t \\
& \leq \int_{0}^{T} \int_{B_{\varrho}(0)}\left(\left|v \cdot \partial_{x} f_{k}\right|+\left|\left(\mathbf{A}+\left(v \times \mathbf{B}_{k}\right)\right) \cdot \partial_{v} f_{k}\right|+\left|\partial_{x} \psi_{f_{k}} \cdot \mathbf{C}_{k}\right|+\left|\chi \Phi_{\mathbf{a}, f_{k}}\right|+\left|\mathbf{b}_{k}\right|\right)^{2} \mathrm{~d} z \mathrm{~d} t
\end{aligned}
$$




$$
\begin{aligned}
& \leq c \int_{0}^{T} \int_{B_{\varrho}(0)} \varrho^{2}\left|\partial_{x} f_{k}(t, z)\right|^{2}+\left(\|\mathbf{A}(t)\|_{\infty}^{2}+\varrho^{2}\left\|\mathbf{B}_{k}(t)\right\|_{\infty}^{2}\right)\left|\partial_{v} f_{k}(t, z)\right|^{2}+c+\left|\mathbf{b}_{k}(t, z)\right|^{2} \mathrm{~d} z \mathrm{~d} t \\
& \leq c .
\end{aligned}
$$

Since all $f_{k}(t)$ are compactly supported in $B_{\varrho}(0)$ this yields

$$
\left\|f_{k}\right\|_{L^{\infty}(] 0, T\left[\times \mathbb{R}^{6}\right)}+\left\|f_{k}\right\|_{H^{1}(] 0, T\left[\times \mathbb{R}^{6}\right)} \leq c .
$$

Recall that due to the Riesz representation theorem $L^{\infty}(] 0, T\left[\times \mathbb{R}^{6}\right)$ can be interpreted as the dual space of $L^{1}(] 0, T\left[\times \mathbb{R}^{6}\right)$ that is denoted by $L^{1}(] 0, T\left[\times \mathbb{R}^{6}\right)^{*}$. Furthermore $L^{1}(] 0, T\left[\times \mathbb{R}^{6}\right)$ can be interpreted as a subset of the dual space $L^{\infty}(] 0, T\left[\times \mathbb{R}^{6}\right)^{*}$.

Then, according to the Banach-Alaoglu theorem, there exists $f \in H^{1}(] 0, T\left[\times \mathbb{R}^{6}\right)$ such that $f_{k} \rightarrow f$ after extraction of a subsequence. Moreover there exists some function $f^{*} \in L^{\infty}(] 0, T\left[\times \mathbb{R}^{6}\right)$ such that $f_{k} \stackrel{*}{\rightarrow} f^{*}$ up to a subsequence. This means that a subsequence of $\left(f_{k}\right)$ converges to $f^{*}$ with respect to the weak-*-topology on $L^{1}(] 0, T\left[\times \mathbb{R}^{6}\right)^{*}$. More precisely, for any $\varphi \in L^{1}(] 0, T\left[\times \mathbb{R}^{6}\right)$,

$$
\int_{0, T\left[\times \mathbb{R}^{6}\right.} f_{n}(t, x) \varphi(t, x) \mathrm{d}(t, x) \rightarrow \int_{] 0, T\left[\times \mathbb{R}^{6}\right.} f^{*}(t, x) \varphi(t, x) \mathrm{d}(t, x), \quad n \rightarrow \infty
$$

up to a subsequence by Riesz' representation theorem. This directly implies that $f=f^{*}$ and consequently $f \in L^{\infty}(] 0, T\left[\times \mathbb{R}^{6}\right) \cap H^{1}(] 0, T\left[\times \mathbb{R}^{6}\right)$.

We will now show that $f$ is a strong solution of (5.1) by verifying the conditions of Definition 25.

Condition (i) is evident since we have already proved that $f \in H^{1}(] 0, T\left[\times \mathbb{R}^{6}\right)$ $\subset W^{1,2}\left(0, T ; L^{2}\right)$ which directly yields $f \in C\left([0, T] ; L^{2}\right)$ by Sobolev's embedding theorem.

Condition (iv) is also obvious because supp $f_{k} \subset B_{\varrho}(0)$ for all $k \in \mathbb{N}, t \in[0, T]$. The radius $\varrho$ does not depend on $k$ and satisfies $\varrho<\zeta(3+r)$.

Condition (ii): By Rellich-Kondrachov, $f_{k} \rightarrow f$ in $L^{2}\left([0, T] \times \mathbb{R}^{6}\right)$ up to a subsequence. Thus for any $\varphi \in C_{c}^{\infty}(] 0, T\left[\times \mathbb{R}^{6}\right)$,

$$
\begin{aligned}
& 0=\int_{[0, T] \times \mathbb{R}^{6}}\left(\partial_{t} f_{k}+v \cdot \partial_{x} f_{k}+\mathbf{A} \cdot \partial_{v} f_{k}+\left(v \times \mathbf{B}_{k}\right) \cdot \partial_{v} f_{k}-\partial_{x} \psi_{f_{k}} \cdot \mathbf{C}_{k}-\chi \Phi_{\mathbf{a}, f_{k}}-\mathbf{b}_{k}\right) \varphi \mathrm{d}(t, z) \\
& \quad \rightarrow \int_{[0, T] \times \mathbb{R}^{6}}\left(\partial_{t} f+v \cdot \partial_{x} f+\mathbf{A} \cdot \partial_{v} f+(v \times \mathbf{B}) \cdot \partial_{v} f-\partial_{x} \psi_{f} \cdot \mathbf{C}-\chi \Phi_{\mathbf{a}, f}-\mathbf{b}\right) \varphi \mathrm{d}(t, z)
\end{aligned}
$$

if $k \rightarrow \infty$. This means (ii) as $\varphi$ was arbitrary.

Condition (iii): Finally, according to Mazur's lemma, there exists some sequence $\left(\bar{f}_{k}\right)_{k \in \mathbb{N}} \subset H^{1}(] 0, T\left[\times \mathbb{R}^{6}\right)$ such that $\bar{f}_{k} \rightarrow f$ in $H^{1}(] 0, T\left[\times \mathbb{R}^{6}\right)$ where for all $k \in \mathbb{N}$, $\bar{f}_{k}$ is a convex combination of $f_{1}, \ldots, f_{k}$. This means $\bar{f}_{k}(0)=\stackrel{\circ}{\mathbf{f}}$ and hence

$$
\|f(0)-\stackrel{\circ}{\mathbf{f}}\|_{L^{2}} \leq c\left\|f-\bar{f}_{k}\right\|_{W^{1,2}\left(0, T ; L^{2}\right)} \leq c\left\|f-\bar{f}_{k}\right\|_{H^{1}(] 0, T\left[\times \mathbb{R}^{6}\right)} \rightarrow 0, \quad k \rightarrow \infty .
$$


Consequently $f$ is a strong solution but we still have to prove uniqueness. We assume that there exists another strong solution $\tilde{f}$ and define $d:=f-\tilde{f}$. Then for all $t \in[0, T]$,

$$
\begin{aligned}
\|d(t)\|_{L^{2}}^{2} & =\int d(t)^{2} \mathrm{~d} z=2 \int_{0}^{t} \int \partial_{t} d(s) d(s) \mathrm{d} z \mathrm{~d} s \\
& =2 \int_{0}^{t} \int-v \cdot \partial_{x} d(s) d(s)-\mathbf{A}(s) \cdot \partial_{v} d(s) d(s)-(v \times \mathbf{B}(s)) \cdot \partial_{v} d(s) d(s) \\
& \quad+\partial_{x} \psi_{d(s)} \cdot \mathbf{C}(s) d(s)+\chi \Phi_{\mathbf{a}, d}(s) d(s) \mathrm{d} z \mathrm{~d} s \\
= & 2 \int_{0}^{t} \int \partial_{x} \psi_{d(s)} \cdot \mathbf{C}(s) d(s)+\chi \Phi_{\mathbf{a}, d}(s) d(s) \mathrm{d} z \mathrm{~d} s \\
\leq & c \int_{0}^{t}\|d(s)\|_{L^{2}}^{2} \mathrm{~d} s
\end{aligned}
$$

Hence $\|f(t)-\tilde{f}(t)\|_{L^{2}}^{2}=\|d(t)\|_{L^{2}}^{2}=0$ for every $t \in[0, T]$ once again by Gronwall's lemma which proves (a).

To prove (b) we only have to approximate $\mathbf{B}$. Therefore we choose some sequence $\left(\mathbf{B}_{k}\right) \subset C\left([0, T] ; C^{1, \gamma}\right)$ such that

$$
\left\|\mathbf{B}_{k}-\mathbf{B}\right\|_{L^{2}\left(0, T ; C^{1, \gamma}\right)} \rightarrow 0, \quad k \rightarrow \infty \quad \text { and } \quad\left\|\mathbf{B}_{k}\right\|_{L^{2}\left(0, T ; C^{1, \gamma}\right)} \leq 2\|\mathbf{B}\|_{L^{2}\left(0, T ; C^{1, \gamma}\right)}, \quad k \in \mathbb{N} .
$$

Then for any $k \in \mathbb{N}$ there exists a unique classical solution $f_{k}$ of the system (5.1) to the coefficients $\mathbf{a}, \mathbf{f}, \mathbf{A}, \mathbf{B}_{k}$ and $\boldsymbol{\chi}$ according to Proposition 24 . Recall that for all $t \in[0, T]$, $\operatorname{supp} f_{k}(t) \subset B_{\varrho}(0)$ where $\varrho:=\zeta(r+1)$ with $r=\max \left\{r_{0}, r_{2}\right\}$. Again, let $Z_{k}$ denote the solution of the characteristic system to $\mathbf{A}$ and $\mathbf{B}_{k}$ satisfying $Z_{k}(t, t, z)=z$ and in the following the letter $c$ denotes some generic positive constant depending only on $T$, $r_{0}, r_{2},\|\mathbf{a}\|_{C\left([0, T] ; C_{b}^{1}\right)},\|\stackrel{f}{\mathbf{f}}\|_{C_{b}^{2}},\|\mathbf{A}\|_{C\left([0, T] ; C^{1, \gamma}\right)},\|\mathbf{B}\|_{L^{2}\left(0, T ; C^{1, \gamma}\right)}$ and $\|\chi\|_{C_{b}^{1}}$. Now for all $s, t \in[0, T]$ (where $s \leq t$ without loss of generality) and $z \in B_{\varrho}(0)$,

$$
\begin{aligned}
\left|Z_{k}(s, t, z)-Z_{j}(s, t, z)\right| \leq & \int_{s}^{t} c\left|Z_{k}(\tau)-Z_{j}(\tau)\right|+\left|\mathbf{A}\left(\tau, Z_{k}(\tau)\right)-\mathbf{A}\left(\tau, Z_{j}(\tau)\right)\right| \\
& +c\left|\mathbf{B}_{k}\left(\tau, Z_{k}(\tau)\right)-\mathbf{B}_{j}\left(\tau, Z_{j}(\tau)\right)\right| \mathrm{d} \tau \\
\leq & \int_{s}^{t} c\left(1+\left\|D_{x} \mathbf{A}(\tau)\right\|_{\infty}+\left\|D_{x} \mathbf{B}_{k}(\tau)\right\|_{\infty}\right)\left|Z_{k}(\tau)-Z_{j}(\tau)\right| \mathrm{d} \tau \\
& +c \int_{0}^{T}\left\|\mathbf{B}_{k}(\tau)-\mathbf{B}_{j}(\tau)\right\|_{\infty} \mathrm{d} \tau
\end{aligned}
$$

which implies that

$$
\left\|Z_{k}(s, t, \cdot)-Z_{j}(s, t, \cdot)\right\|_{\infty} \leq c\left\|\mathbf{B}_{k}-\mathbf{B}_{j}\right\|_{L^{2}\left(0, T ; L^{\infty}\right)} .
$$


For any $i \in\{1, \ldots, 6\}$ the difference of the $i$-th derivative can be bounded in the following manner:

$$
\begin{aligned}
& \left|\partial_{z_{i}} Z_{k}(s)-\partial_{z_{i}} Z_{j}(s)\right| \leq \int_{s}^{t}\left[\left|\partial_{z_{i}} V_{k}(\tau)-\partial_{z_{i}} V_{j}(\tau)\right|\right. \\
& +\left|D_{x} \mathbf{A}\left(\tau, X_{k}(\tau)\right) \partial_{z_{i}} X_{k}(\tau)-D_{x} \mathbf{A}\left(\tau, X_{j}(\tau)\right) \partial_{z_{i}} X_{j}(\tau)\right| \\
& +\left|V_{k}(\tau)-V_{j}(\tau)\right|\left|D_{x} \mathbf{B}_{k}\left(\tau, X_{k}(\tau)\right) \partial_{z_{i}} X_{k}(\tau)\right| \\
& +\left|\partial_{z_{i}} V_{k}(\tau)-\partial_{z_{i}} V_{j}(\tau)\right|\left|\mathbf{B}_{k}\left(\tau, X_{k}(\tau)\right)\right| \\
& +c\left|D_{x} \mathbf{B}_{k}\left(\tau, X_{k}(\tau)\right) \partial_{z_{i}} X_{k}(\tau)-D_{x} \mathbf{B}_{j}\left(\tau, X_{j}(\tau)\right) \partial_{z_{i}} X_{j}(\tau)\right| \\
& \left.+c\left|\mathbf{B}_{k}\left(\tau, X_{k}(\tau)\right) \partial_{z_{i}} X_{k}(\tau)-\mathbf{B}_{j}\left(\tau, X_{j}(\tau)\right) \partial_{z_{i}} X_{j}(\tau)\right|\right] \mathrm{d} \tau \\
& \leq \int_{s}^{t}\left[c\left(1+\|\mathbf{A}(\tau)\|_{C^{1, \gamma}}+\left\|\mathbf{B}_{k}(\tau)\right\|_{C^{1, \gamma}}\right)\left|\partial_{z_{i}} Z_{k}(\tau)-\partial_{z_{i}} Z_{j}(\tau)\right|\right. \\
& +c\left(1+\|\mathbf{A}(\tau)\|_{C^{1, \gamma}}+\left\|\mathbf{B}_{k}(\tau)\right\|_{C^{1, \gamma}}\right)\left\|Z_{k}(\tau)-Z_{j}(\tau)\right\|_{\infty}^{\gamma} \\
& \left.+c\left\|\mathbf{B}_{k}(\tau)-\mathbf{B}_{j}(\tau)\right\|_{C_{b}^{1}}\right] \mathrm{d} \tau \\
& \leq \int_{s}^{t} c\left(1+\|\mathbf{A}(\tau)\|_{C^{1, \gamma}}+\left\|\mathbf{B}_{k}(\tau)\right\|_{C^{1, \gamma}}\right)\left|\partial_{z_{i}} Z_{k}(\tau)-\partial_{z_{i}} Z_{j}(\tau)\right| \mathrm{d} \tau \\
& +c\left\|\mathbf{B}_{k}-\mathbf{B}_{j}\right\|_{L^{2}\left(0, T ; C_{b}^{1}\right)}^{\gamma}
\end{aligned}
$$

for all $s, t \in[0, T]$ and $z \in B_{\varrho}(0)$. Thus

$$
\left\|\partial_{z} Z_{k}(s)-\partial_{z} Z_{j}(s)\right\|_{L^{\infty}\left(B_{r}(0)\right)} \leq c\left\|\mathbf{B}_{k}-\mathbf{B}_{j}\right\|_{L^{2}\left(0, T ; C_{b}^{1}\right)}^{\gamma} .
$$

Now for all $t \in[0, T], z \in B_{\varrho}(0)$,

$$
\begin{aligned}
\left|f_{k}(t, z)-f_{j}(t, z)\right| & \leq\|D \stackrel{\circ}{\mathbf{f}}\|_{\infty}\left|Z_{k}(0, t, z)-Z_{j}(0, t, z)\right|+\int_{0}^{t} c\left\|f_{k}(\tau)-f_{j}(\tau)\right\|_{\infty} \mathrm{d} \tau \\
& \leq c\left\|\mathbf{B}_{k}-\mathbf{B}_{j}\right\|_{L^{2}\left(0, T ; L^{\infty}\right)}+c \int_{0}^{t}\left\|f_{k}(\tau)-f_{j}(\tau)\right\|_{\infty} \mathrm{d} \tau
\end{aligned}
$$

and thus Gronwall's lemma implies that

$$
\left\|f_{k}-f_{j}\right\|_{L^{\infty}\left(0, T ; L^{\infty}\right)} \leq c\left\|\mathbf{B}_{k}-\mathbf{B}_{j}\right\|_{L^{2}\left(0, T ; L^{\infty}\right)}
$$


Moreover for all $t \in[0, T], z \in B_{r}(0)$,

$$
\begin{aligned}
& \left|\partial_{z} f_{k}(t, z)-\partial_{z} f_{j}(t, z)\right| \\
& \leq\left\|D^{2} \mathbf{f}\right\|_{\infty}\left|\partial_{z} Z_{k}(0, t, z)-\partial_{z} Z_{j}(0, t, z)\right|+\int_{0}^{t} c\left\|\partial_{z} f_{k}(\tau)-\partial_{z} f_{j}(\tau)\right\|_{\infty} \mathrm{d} \tau \\
& \quad+\int_{0}^{t} c\left\|f_{k}(\tau)-f_{j}(\tau)\right\|_{\infty} \mathrm{d} \tau \\
& \leq c\left\|\mathbf{B}_{k}-\mathbf{B}_{j}\right\|_{L^{2}\left(0, T ; C_{b}^{1}\right)}^{\gamma}+c \int_{0}^{t}\left\|\partial_{z} f_{k}(\tau)-\partial_{z} f_{j}(\tau)\right\|_{\infty} \mathrm{d} \tau
\end{aligned}
$$

and consequently

$$
\left\|\partial_{z} f_{k}-\partial_{z} f_{j}\right\|_{L^{\infty}\left(0, T ; L^{\infty}\right)} \leq c\left\|\mathbf{B}_{k}-\mathbf{B}_{j}\right\|_{L^{2}\left(0, T ; C^{1, \gamma}\right)}^{\gamma}
$$

Similar to (A.11) we can easily conclude that

$$
\left\|\partial_{t} f_{k}-\partial_{t} f_{j}\right\|_{L^{2}\left(0, T ; C_{b}\right)} \leq c\left\|\mathbf{B}_{k}-\mathbf{B}_{j}\right\|_{L^{2}\left(0, T ; C^{1, \gamma}\right)}^{\gamma} .
$$

This means that $\left(f_{k}\right)$ is a Cauchy sequence in $W^{1,2}\left(0, T ; C_{b}\right) \cap C\left([0, T] ; C_{b}^{1}\right)$ and thus it converges to some function $f \in W^{1,2}\left(0, T ; C_{b}\right) \cap C\left([0, T] ; C_{b}^{1}\left(\mathbb{R}^{6}\right)\right)$ because of completeness. Note that for all $t \in[0, T], \operatorname{supp} f(t) \subset B_{\zeta(r+2)}$. One can easily show that $f$ satisfies the system (5.1) almost everywhere and thus $f$ is a strong solution due to Definition 25.

Moreover, by the definition of convergence, we can find $k \in \mathbb{N}$ such that

$$
\left\|f-f_{k}\right\|_{W^{1,2}\left(0, T ; C_{b}\right)}+\left\|f-f_{k}\right\|_{C\left(0, T ; C_{b}^{1}\right)} \leq 1
$$

and consequently

$$
\begin{aligned}
& \|f\|_{W^{1,2}\left(0, T ; C_{b}\right)}+\|f\|_{C\left(0, T ; C_{b}^{1}\right)} \\
& \quad \leq\left\|f-f_{k}\right\|_{W^{1,2}\left(0, T ; C_{b}\right)}+\left\|f-f_{k}\right\|_{C\left(0, T ; C_{b}^{1}\right)}+\left\|f_{k}\right\|_{W^{1,2}\left(0, T ; C_{b}\right)}+\left\|f_{k}\right\|_{C\left(0, T ; C_{b}^{1}\right)} \\
& \quad \leq\left\|f-f_{k}\right\|_{W^{1,2}\left(0, T ; C_{b}\right)}+\left\|f-f_{k}\right\|_{C\left(0, T ; C_{b}^{1}\right)}+c\left\|f_{k}\right\|_{C_{b}^{1}(] 0, T\left[\times \mathbb{R}^{6}\right)} \\
& \quad \leq c .
\end{aligned}
$$

as the sequence $\left(f_{k}\right)$ is bounded in $C_{b}^{1}(] 0, T\left[\times \mathbb{R}^{6}\right)$ according to Proposition 24 and the bound $\left\|\mathbf{B}_{k}\right\|_{L^{2}\left(0, T ; C^{1, \gamma}\right)} \leq 2\|\mathbf{B}\|_{L^{2}\left(0, T ; C^{1, \gamma}\right)}$.

We will now assume that $r_{1}=\zeta\left(r_{0}\right)$. As it has already been discussed in the comment to Proposition 24 the values of $\left.f_{k}\right|_{B_{r_{0}}(0)}$ do not depend on the choice of $\chi$ as long as $\chi=1$ on $B_{r_{1}}(0)$. As $\left.f_{k}\right|_{B_{r_{0}}(0)}$ converges to $\left.f\right|_{B_{r_{0}}(0)}$ uniformely on $[0, T] \times B_{r_{0}}(0)$ this result holds true for $\left.f\right|_{B_{r_{0}}(0)}$. 
Proof of Theorem 31 Step 1: Obviously the system (6.6) has a unique strong solution $g_{B}$ in the sense of Corollary 26 (a). Unfortunately the coefficients do not satisfy the stronger regularity conditions (5.11) of Corollary 26 (b) as the final value $f_{B}(T)-f_{d}$ is not in $C_{c}^{2}\left(\mathbb{R}^{6}\right)$. However, because of linearity, it holds that $g_{B}=\tilde{g}_{B}-h_{B}$ where $\tilde{g}_{B}$ is a solution of

$$
\left\{\begin{array}{l}
\partial_{t} \tilde{g}+v \cdot \partial_{x} \tilde{g}-\partial_{x} \psi_{f_{B}} \cdot \partial_{v} \tilde{g}+(v \times B) \cdot \partial_{v} \tilde{g}=\Phi_{f_{B}, \tilde{g}} \chi \\
\left.\tilde{g}\right|_{t=T}=f(T)
\end{array}\right.
$$

and $h_{B}$ is a solution of

$$
\left\{\begin{array}{l}
\partial_{t} h+v \cdot \partial_{x} h-\partial_{x} \psi_{f_{B}} \cdot \partial_{v} h+(v \times B) \cdot \partial_{v} h=\Phi_{f_{B}, h} \chi \\
\left.h\right|_{t=T}=f_{d}
\end{array}\right.
$$

Now the first system has a unique strong solution in the sense of Corollary 26 (a) and the second one possesses a strong solution in the sense of Corollary 26 (b) since $f_{d} \in C_{c}^{2}\left(\mathbb{R}^{6}\right)$. Indeed the solution $\tilde{g}_{B}$ is much more regular. As $\Phi_{f_{B}, f_{B}}=0$ one can easily see that $f_{B}$ is a solution of the first system and thus, because of uniqueness, $\tilde{g}_{B}=f_{B}$. Consequently $g_{B}=f_{B}-h_{B} \in W^{1,2}\left(0, T ; C_{b}\right) \cap C\left([0, T] ; C_{b}^{1}\right)$. Due to Corollary 26 (b) and Lemma 15, the values that $h_{B}$ takes on $B_{R}(0)$ do not depend on the choice of $\chi$. Of course $f_{B}$ does not depend on $\chi$ either and hence $\left.g_{B}\right|_{B_{R}(0)}$ does not depend on the choice of $\chi$.

Step 2: We will now prove the Hölder estimate. It suffices to establish the result for $h$. as the result has already been proved for $f$. in Corollary 21. Therefore let $B, H \in \mathbb{B}_{K}$ be arbitrary and let $C>0$ denote some generic constant depending only on $\stackrel{\circ}{f}, f_{d}, T, K$, $\beta$ and $\|\chi\|_{C_{b}^{2}}$. According to Lemma 10 (d) there exist sequences $\left(B_{k}\right),\left(H_{k}\right) \subset \mathbb{M}$ such that

$$
\left\|B_{k}-B\right\|_{L^{2}\left(0, T ; W^{2, \beta}\right)} \rightarrow 0, \quad\left\|H_{k}-H\right\|_{L^{2}\left(0, T ; W^{2, \beta}\right)} \rightarrow 0, \quad k \rightarrow \infty .
$$

By Corollary $26(\mathrm{~b})$ the induced classical solutions $h_{B_{k}}$ and $h_{H_{k}}$ satisfy

$$
\begin{gathered}
h_{B_{k}} \rightarrow h_{B}, h_{H_{k}} \rightarrow h_{H} \quad \text { in } W^{1,2}\left(0, T ; C_{b}\right) \cap C\left([0, T] ; C_{b}^{1}\right), \\
\left\|h_{B_{k}}\right\|_{W^{1,2}\left(0, T ; C_{b}\right)}+\left\|h_{B_{k}}\right\|_{C\left([0, T] ; C_{b}^{1}\right)} \leq C \text { and }\left\|h_{H_{k}}\right\|_{W^{1,2}\left(0, T ; C_{b}\right)}+\left\|h_{H_{k}}\right\|_{C\left([0, T] ; C_{b}^{1}\right)} \leq C .
\end{gathered}
$$

Note that the constant $C$ does not depend on $k$ since $\left\|B_{k}\right\|_{L^{2}\left(0, T, W^{2, \beta}\right)}$ and $\left\|H_{k}\right\|_{L^{2}\left(0, T, W^{2, \beta}\right)}$ are bounded by $2 K$. Also note that there exists some constant $\varrho>0$ depending only on $\stackrel{\circ}{f}, f_{d}, T, K$ and $\beta$ (but not on $k$ ) such that $\operatorname{supp} h_{B_{k}} \subset B_{\varrho}(0)$ and $\operatorname{supp} h_{H_{k}} \subset B_{\varrho}(0)$.

As $h_{B_{k}}$ and $h_{H_{k}}$ are classical solutions they satisfy the implicit representation formula (5.8). We also know from Lemma 16 and its proof (with $\varrho$ instead of $R$ ) that

$$
\begin{aligned}
&\left\|f_{B_{k}}(t)-f_{H_{k}}(t)\right\|_{\infty} \leq C\left\|B_{k}-H_{k}\right\|_{L^{2}\left(0, T ; W^{2, \beta}\right)}, \\
&\left\|D_{z} f_{B_{k}}(t)-D_{z} f_{H_{k}}(t)\right\|_{\infty} \leq C\left\|B_{k}-H_{k}\right\|_{L^{2}\left(0, T ; W^{2, \beta}\right)}^{\gamma}, \\
&\left\|Z_{B_{k}}(t)-Z_{H_{k}}(t)\right\|_{L^{\infty}\left(B_{\varrho}(0)\right)} \leq C\left\|B_{k}-H_{k}\right\|_{L^{2}\left(0, T ; W^{2, \beta}\right)} \\
&\left\|D_{z} Z_{B_{k}}(t)-D_{z} Z_{H_{k}}(t)\right\|_{L^{\infty}\left(B_{\varrho}(0)\right)} \leq C\left\|B_{k}-H_{k}\right\|_{L^{2}\left(0, T ; W^{2, \beta}\right)}^{\gamma}
\end{aligned}
$$

for all $t \in[0, T]$. 
Together with Lemma 15 this yields

$$
\begin{aligned}
& \left\|h_{B_{k}}(t)-h_{H_{k}}(t)\right\|_{L^{\infty}} \\
& \leq\left\|f_{d}\right\|_{C_{b}^{1}}\left\|Z_{B_{k}}(T, t, \cdot)-Z_{H_{k}}(T, t, \cdot)\right\|_{L^{\infty}\left(B_{\varrho}(0)\right)} \\
& \quad+\int_{t}^{T}\left\|\Phi_{{B_{B}}_{k}, h_{B_{k}}}\left(s, Z_{B_{k}}(s, t, \cdot)\right)-\Phi_{f_{H_{k}}, h_{H_{k}}}\left(s, Z_{H_{k}}(s, t, \cdot)\right)\right\|_{L^{\infty}\left(B_{\varrho}(0)\right)} \mathrm{d} s \\
& \leq C\left\|B_{k}-H_{k}\right\|_{L^{2}\left(0, T ; W^{2, \beta}\right)} \\
& \quad+C \int_{t}^{T}\left\|h_{B_{k}}(s)\right\|_{L^{\infty}}\left\|D_{z} f_{B_{k}}(s)\right\|_{L \infty}\left\|Z_{B_{k}}(s)-Z_{H_{k}}(s)\right\|_{L^{\infty}\left(B_{\varrho}(0)\right)} \mathrm{d} s \\
& \quad+C \int_{t}^{T}\left\|h_{B_{k}}(s)\right\|_{L^{2}}\left\|\partial_{v} f_{B_{k}}(s)-\partial_{v} f_{H_{k}}(s)\right\|_{L^{\infty}} \mathrm{d} s \\
& \quad+C \int_{t}^{T}\left\|h_{B_{k}}(s)-h_{B_{k}}(s)\right\|_{L^{2}}\left\|\partial_{v} f_{H_{k}}(s)\right\|_{L^{\infty}} \mathrm{d} s \\
& \quad C\left\|B_{k}-H_{k}\right\|_{L^{2}\left(0, T ; W^{2, \beta}\right)}^{\gamma}+C \int_{t}^{T}\left\|h_{B_{k}}(s)-h_{B_{k}}(s)\right\|_{L^{2}} \mathrm{~d} s
\end{aligned}
$$

and hence $\left\|h_{B_{k}}-h_{H_{k}}\right\|_{C\left([0, T] ; C_{b}\right)} \leq C\left\|B_{k}-H_{k}\right\|_{L^{2}\left(0, T ; W^{2, \beta}\right)}^{\gamma}$. Furthermore,

$\left\|\partial_{z} h_{B_{k}}(t)-\partial_{z} h_{H_{k}}(t)\right\|_{L^{\infty}}$

$\leq C\left\|f_{d}\right\|_{C_{b}^{2}}\left\|Z_{B_{k}}(T, t, \cdot)-Z_{H_{k}}(T, t, \cdot)\right\|_{W^{1, \infty}\left(B_{\varrho}(0)\right)}$

$+C \int_{t}^{T}\left\|\Phi_{{f_{B}}_{k}, h_{B_{k}}}^{\prime}\left(s, Z_{B_{k}}(s)\right)\right\|_{L^{\infty}}\left\|Z_{B_{k}}(s)-Z_{H_{k}}(s)\right\|_{W^{1, \infty}\left(B_{\varrho}(0)\right)} \mathrm{d} s$

$+C \int_{t}^{T}\left\|\Phi_{f_{B_{k}}, h_{B_{k}}}\left(s, Z_{B_{k}}(s)\right)\right\|_{L^{\infty}}\left\|Z_{B_{k}}(s)-Z_{H_{k}}(s)\right\|_{W^{1, \infty}\left(B_{\varrho}(0)\right)} \mathrm{d} s$

$+C \int_{t}^{T}\left\|\Phi_{{B_{B}}_{k}, h_{B_{k}}}^{\prime}\left(s, Z_{B_{k}}(s)\right)-\Phi_{f_{H_{k}}, h_{H_{k}}}^{\prime}\left(s, Z_{H_{k}}(s)\right)\right\|_{L^{\infty}\left(B_{\varrho}(0)\right)}\left\|Z_{H_{k}}(s)\right\|_{W^{1, \infty}\left(B_{\varrho}(0)\right)} \mathrm{d} s$

$+C \int_{t}^{T}\left\|\Phi_{f_{B_{k}}, h_{B_{k}}}\left(s, Z_{B_{k}}(s)\right)-\Phi_{f_{H_{k}}, h_{H_{k}}}\left(s, Z_{H_{k}}(s)\right)\right\|_{L^{\infty}\left(B_{\varrho}(0)\right)}\left\|Z_{H_{k}}(s)\right\|_{W^{1, \infty}\left(B_{\varrho}(0)\right)} \mathrm{d} s$ 


$$
\begin{aligned}
\leq & C\left\|B_{k}-H_{k}\right\|_{L^{2}\left(0, T ; W^{2, \beta}\right)}^{\gamma} \\
& +C \int_{t}^{T}\left\|h_{B_{k}}(s)\right\|_{W^{1 \infty}}\left\|f_{B_{k}}(s)\right\|_{W^{1 \infty}}\left\|Z_{B_{k}}(s)-Z_{H_{k}}(s)\right\|_{W^{1, \infty}\left(B_{\varrho}(0)\right)} \mathrm{d} s \\
& +C \int_{t}^{T}\left\|h_{B_{k}}(s)\right\|_{W^{1, \infty}}\left\|f_{B_{k}}(s)-f_{H_{k}}(s)\right\|_{W^{1, \infty}}\left\|Z_{H_{k}}(s)\right\|_{W^{1, \infty}\left(B_{\varrho}(0)\right)} \mathrm{d} s \\
& +C \int_{t}^{T}\left\|h_{B_{k}}(s)-h_{B_{k}}(s)\right\|_{W^{1, \infty}}\left\|f_{H_{k}}(s)\right\|_{W^{1, \infty}}\left\|Z_{H_{k}}(s)\right\|_{W^{1, \infty}\left(B_{\varrho}(0)\right)} \mathrm{d} s \\
\leq C & \left\|B_{k}-H_{k}\right\|_{L^{2}\left(0, T ; W^{2, \beta}\right)}^{\gamma}+C \int_{t}^{T}\left\|\partial_{z} h_{B_{k}}(s)-\partial_{z} h_{B_{k}}(s)\right\|_{L^{2}} \mathrm{~d} s
\end{aligned}
$$

and consequently $\left\|\partial_{z} h_{B_{k}}(t)-\partial_{z} h_{H_{k}}\right\|_{C\left([0, T] ; C_{b}\right)} \leq C\left\|B_{k}-H_{k}\right\|_{L^{2}\left(0, T ; W^{2, \beta}\right)}^{\gamma}$ by Gronwall. The difference of the $t$-derivatives can be bounded by

$$
\begin{aligned}
& \left\|\partial_{t} h_{B_{k}}(t)-\partial_{t} h_{H_{k}}(t)\right\|_{L^{\infty}} \\
& \quad \leq \varrho\left\|\partial_{x} h_{B_{k}}(t)-\partial_{x} h_{H_{k}}(t)\right\|_{L^{\infty}}+\left\|\partial_{x} \psi_{f_{B_{k}}}\right\|_{\infty}\left\|\partial_{v} h_{B_{k}}(t)-\partial_{v} h_{H_{k}}(t)\right\|_{L^{\infty}} \\
& \quad+\left\|\partial_{x} \psi_{f_{B_{k}}}-\partial_{x} \psi_{f_{H_{k}}}\right\|_{\infty}\left\|\partial_{v} h_{H_{k}}(t)\right\|_{L^{\infty}}+\varrho\left\|B_{k}(t)\right\|_{\infty}\left\|\partial_{v} h_{B_{k}}(t)-\partial_{v} h_{H_{k}}(t)\right\|_{L^{\infty}} \\
& \quad+\varrho\left\|B_{k}(t)-H_{k}(t)\right\|_{\infty}\left\|\partial_{v} h_{H_{k}}(t)\right\|_{L^{\infty}}+\left\|\Phi_{B_{B_{k}}, h_{B_{k}}-h_{H_{k}}}(t)\right\|_{L^{\infty}\left(B_{\varrho}(0)\right)} \\
& \quad+\left\|\Phi_{f_{B_{k}}-f_{H_{k}}, h_{H_{k}}}(t)\right\|_{L^{\infty}\left(B_{\varrho}(0)\right)} \\
& \quad \leq C\left(1+\left\|B_{k}(t)\right\|_{\infty}\right)\left\|B_{k}-H_{k}\right\|_{L^{2}\left(0, T ; W^{2, \beta}\right)}^{\gamma}+C\left\|B_{k}(t)-H_{k}(t)\right\|_{W^{2, \beta}}
\end{aligned}
$$

from which we can conclude that $\left\|\partial_{t} h_{B_{k}}-\partial_{t} h_{H_{k}}\right\|_{L^{2}\left(0, T ; C_{b}\right)} \leq C\left\|B_{k}-H_{k}\right\|_{L^{2}\left(0, T ; W^{2, \beta}\right)}^{\gamma}$. In summary we have established that

$$
\left\|h_{B_{k}}-h_{H_{k}}\right\|_{W^{1,2}\left(0, T ; C_{b}\right)}+\left\|h_{B_{k}}-h_{H_{k}}\right\|_{C\left([0, T] ; C_{b}^{1}\right)} \leq C\left\|B_{k}-H_{k}\right\|_{L^{2}\left(0, T ; W^{2, \beta}\right)}^{\gamma} .
$$

For $k \rightarrow \infty$ this directly yields

$$
\left\|h_{B}-h_{H}\right\|_{W^{1,2}\left(0, T ; C_{b}\right)}+\left\|h_{B}-h_{H}\right\|_{C\left([0, T] ; C_{b}^{1}\right)} \leq C\|B-H\|_{L^{2}\left(0, T ; W^{2, \beta}\right)}^{\gamma} .
$$

and hence

$$
\left\|g_{B}-g_{H}\right\|_{W^{1,2}\left(0, T ; C_{b}\right)}+\left\|g_{B}-g_{H}\right\|_{C\left([0, T] ; C_{b}^{1}\right)} \leq C\|B-H\|_{L^{2}\left(0, T ; W^{2, \beta}\right)}^{\gamma} .
$$

Step 3: We must still prove that $g_{B} \in L^{\infty}\left(0, T ; H^{2}\right)$. Since $f_{B} \in L^{\infty}\left(0, T ; H^{2}\right)$ has already been established in Theorem 19 it suffices to show that $h_{B}$ is twice weakly differentiable with respect to $z$ and $D_{z}^{2} h_{B} \in L^{\infty}\left(0, T ; L^{2}\right)$. Recall that for any $k \in \mathbb{N}$, $f_{B_{k}} \in C\left([0, T] ; C_{b}^{2}\right)$ according to Corollary 14 and $h_{B_{k}} \in C\left([0, T] ; C_{b}^{1}\right)$ according to Theorem 24. Thus for all $i \in\{1,2,3\}$,

$$
\partial_{x_{i}} \partial_{v_{i}} f_{B_{k}} h_{B_{k}}+\partial_{v_{i}} f_{B_{k}} \partial_{x_{i}} h_{B_{k}} \in C\left([0, T] ; C_{b}\right),
$$

$\operatorname{supp}\left[\partial_{x_{i}} \partial_{v_{i}} f_{B_{k}} h_{B_{k}}+\partial_{v_{i}} f_{B_{k}} \partial_{x_{i}} h_{B_{k}}\right](t) \subset B_{R}(0), \quad$ for all $t \in[0, T]$. 
Thus by Lemma 6 and Proposition 8,

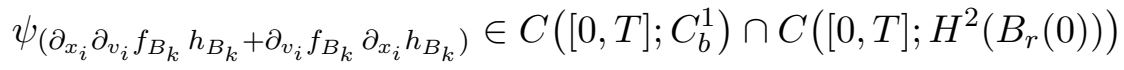

and consequently

$$
\begin{aligned}
& \Phi_{f_{B_{k}}, h_{B_{k}}}=\sum_{i=1}^{3} \partial_{x_{i}} \psi_{\partial_{v_{i}} f_{B_{k}}, h_{B_{k}}} \\
& =\sum_{i=1}^{3} \psi_{\left(\partial_{x_{i}} \partial_{v_{i}} f_{B_{k}} h_{B_{k}}+\partial_{v_{i}} f_{B_{k}} \partial_{x_{i}} h_{B_{k}}\right)} \in C\left([0, T] ; C_{b}^{1}\right) \cap C\left([0, T] ; H^{2}\left(B_{r}(0)\right)\right),
\end{aligned}
$$

for any $r>0$. Hence

$$
\Phi_{f_{B_{k}}, h_{B_{k}}} \chi \in C\left([0, T] ; C_{b}^{1}\right) \cap C\left([0, T] ; H^{2}\right)
$$

since $\chi$ is compactly supported. We also know from Lemma 15 (which holds true with $\varrho$ instead of $R$ ) that $Z_{B_{k}}$ is twice continuously differentiable with respect to $z$ and for all $s \in[0, T]$,

$$
\left\|\left[t \mapsto Z_{B_{k}}(s, t, \cdot)\right]\right\|_{L^{\infty}\left(0, T ; H^{2}\left(B_{\varrho}(0)\right)\right)} \leq C .
$$

Now recall the implicit representation formula (5.8) for $h_{B_{k}}$ that is

$$
h_{B_{k}}(t, z)=f_{d}\left(Z_{B_{k}}(T, t, z)\right)-\int_{t}^{T}\left[\Phi_{f_{B_{k}}, h_{B_{k}}} \chi\right]\left(s, Z_{B_{k}}(s, t, z)\right) \mathrm{d} s
$$

for all $(t, z) \in[0, T] \times \mathbb{R} 6$. As $f_{d} \in C_{c}^{2}\left(\mathbb{R}^{6}\right)$ and $Z_{B_{k}}(T, t, \cdot) \in C^{2}\left(\mathbb{R}^{6}\right)$, the term $f_{d}\left(Z_{B_{k}}(T, t, z)\right)$ is twice continuously differentiable with respect to $z$ by chain rule. The second term of the right-hand side of (A.13) is once continuously differentiable with

$$
\begin{aligned}
\frac{\partial}{\partial z_{j}} \int_{t}^{T}\left[\Phi_{f_{B_{k}}, h_{B_{k}}} \chi\right]\left(s, Z_{B_{k}}(s)\right) \mathrm{d} s= & \int_{t}^{T}\left[\Phi_{f_{B_{k}}, h_{B_{k}}}^{\prime} \chi\right]\left(s, Z_{B_{k}}(s)\right) \cdot \partial_{z_{j}} X_{B_{k}}(s) \mathrm{d} s \\
& +\int_{t}^{T}\left[\Phi_{f_{B_{k}}, h_{B_{k}}} \partial_{z} \chi\right]\left(s, Z_{B_{k}}(s)\right) \cdot \partial_{z_{j}} Z_{B_{k}}(s) \mathrm{d} s
\end{aligned}
$$

Obviously the second summand on the right-hand side of (A.14) is once more continuously differentiable. Moreover,

$$
F:=\Phi_{{f_{B}}_{k}, h_{B_{k}}}^{\prime} \chi \in C\left([0, T] ; H^{1}\right)
$$

and thus we can choose some sequence $\left(F_{m}\right) \subset C\left([0, T] ; C_{b}^{1}\right)$ such that $F_{m} \rightarrow F$ in $C\left([0, T] ; H^{1}\right)$. As $Z_{B_{k}}(s, t, \cdot)$ is a measure preserving $C^{1}$-diffeomorphism according to Lemma 11 it also holds that

$$
\left\|F_{m}\left(s, Z_{B_{k}}(s, t, \cdot)\right)-F\left(s, Z_{B_{k}}(s, t, \cdot)\right)\right\|_{H^{1}}=\left\|F_{m}(s, \cdot)-F(s, \cdot)\right\|_{H^{1}} \rightarrow 0, \quad m \rightarrow \infty
$$


uniformely in $s \in[0, T]$. Consequently,

$$
\int_{t}^{T}\left[\Phi_{{B_{B}}, h_{B_{k}}}^{\prime} \chi\right]\left(s, Z_{B_{k}}(s)\right) \cdot \partial_{z_{i}} X_{B_{k}}(s) \mathrm{d} s=\lim _{m \rightarrow \infty} \int_{t}^{T} F_{m}\left(s, Z_{B_{k}}(s)\right) \cdot \partial_{z_{i}} X_{B_{k}}(s) \mathrm{d} s
$$

in $L^{2}\left(\mathbb{R}^{6}\right)$ and

$$
\begin{aligned}
\frac{\partial}{\partial z_{i}} \int_{t}^{T} F_{m}\left(s, Z_{B_{k}}(s)\right) \cdot \partial_{z_{j}} X_{B_{k}}(s) \mathrm{d} s= & \int_{t}^{T}\left[D_{z} F_{m}\left(s, Z_{B_{k}}(s)\right) \partial_{z_{i}} Z_{B_{k}}(s)\right] \cdot \partial_{z_{j}} X_{B_{k}}(s) \mathrm{d} s \\
& +\int_{t}^{T}\left[F_{m}\left(s, Z_{B_{k}}(s)\right)\right] \cdot \partial_{z_{i}} \partial_{z_{j}} X_{B_{k}}(s) \mathrm{d} s \\
\rightarrow & \int_{t}^{T}\left[D_{z} F\left(s, Z_{B_{k}}(s)\right) \partial_{z_{i}} Z_{B_{k}}(s)\right] \cdot \partial_{z_{j}} X_{B_{k}}(s) \mathrm{d} s \\
& +\int_{t}^{T}\left[F\left(s, Z_{B_{k}}(s)\right)\right] \cdot \partial_{z_{i}} \partial_{z_{j}} X_{B_{k}}(s) \mathrm{d} s
\end{aligned}
$$

in $L^{2}\left(\mathbb{R}^{6}\right)$ if $m \rightarrow \infty$ where $D_{z} F$ is the weak derivative of $F$. From this we can conclude that the right-hand side of (A.13) is twice weakly differentiable with respect to $z$ and hence the same holds for the left-hand side that is $h_{B_{k}}$. It also follows that the weak partial derivatives can be computed by the chain rule formula (if necessary with weak instead of classical derivatives). Thus for any $i, j \in\{1,2,3\}$ the weak derivative $\partial_{z_{i}} \partial_{z_{j}} h_{B_{k}}$ can be bounded by

$$
\begin{aligned}
\left\|\partial_{z_{i}} \partial_{z_{j}} h_{B_{k}}(t)\right\|_{L^{2}} \leq & C\left\|f_{d}\right\|_{C_{b}^{2}}\left\|Z_{B_{k}}(0, t, \cdot)\right\|_{H^{2}\left(B_{\varrho}(0)\right)} \\
& +C \int_{t}^{T}\left\|\Phi_{f_{B_{k}}, h_{B_{k}}}(s)\right\|_{H^{2}\left(B_{\varrho}(0)\right)}\left\|\partial_{z} Z_{B_{k}}(s, t, \cdot)\right\|_{L^{\infty}\left(B_{\varrho}(0)\right)}^{2} \mathrm{~d} s \\
& +C \int_{t}^{T}\left\|\Phi_{f_{B_{k}}, h_{B_{k}}}(s)\right\|_{W^{1, \infty}\left(B_{\varrho}(0)\right)}\left\|Z_{B_{k}}(s, t, \cdot)\right\|_{H^{2}\left(B_{\varrho}(0)\right)} \mathrm{d} s \\
\leq & C+C\left\|Z_{B_{k}}(0, t, \cdot)\right\|_{H^{2}\left(B_{\varrho}(0)\right)}+C \int_{0}^{T}\left\|Z_{B_{k}}(s, t, \cdot)\right\|_{H^{2}\left(B_{\varrho}(0)\right)} \mathrm{d} s .
\end{aligned}
$$

By (A.12) this finally yields

$$
\left\|\partial_{z_{i}} \partial_{z_{j}} h_{B_{k}}\right\|_{L^{\infty}\left(0, T ; L^{2}\right)}^{2} \leq C .
$$

This implies that $\left(\partial_{z_{i}} \partial_{z_{j}} h_{B_{k}}\right)$ is converging with respect to the weak-*-topology on $\left[L^{1}\left(0, T ; L^{2}\right)\right]^{*}=L^{\infty}\left(0, T ; L^{2}\right)$ up to a subsequence. Because of uniqueness, the weak-*limit of the sequence $\left(\partial_{z_{i}} \partial_{z_{j}} h_{B_{k}}\right)$ must be $\partial_{z_{i}} \partial_{z_{j}} h_{B}$ and especially $h_{B} \in L^{\infty}\left(0, T ; H^{2}\right)$. This completes the proof. 


\section{Bibliography}

[1] J. Batt. Global symmetric solutions of the initial value problem in stellar dynamics. J. Differential Equations, 25 (1977), 342-364.

[2] S. S. Dragomir. Some Gronwall Type Inequalities and Applications. Nova Biomedical (2003).

[3] L. C. Evans. Partial Differential Equations (Second Edition). Amer. Math. Soc., Grad. Stud. in Math. Vol. 19 (2010), 284-285.

[4] D. Gilbarg and N. S. Trudinger. Elliptic Partial Differential Equations of Second Order. Springer, 3 (2001).

[5] R. Kurth. Das Anfangswertproblem der Stellardynamik. Z. Astrophys., 30 (1952), 213-229.

[6] E. H. Lieb and M. Loss. Analysis (Second Edition). Amer. Math. Soc., 14 (2001).

[7] A. Lindner. $C^{k}$-Regularität der Lösungen des Vlasov-Poisson-Systems partieller Differentialgleichungen. Diplomarbeit, LMU München (1991).

[8] P.-L. Lions and B. Perthame. Propagation of moments and regularity for the 3dimensional Vlasov-Poisson system. Invent. Math., 105 (1991), 415-430.

[9] R. Steinbauer G. Teschl M. Kunzinger, G. Rein. On classical solutions of the relativistic Vlasov-Klein-Gordon system. Electronic J. Differential Equations Vol. 2005, No. 01 (2005), 1-17.

[10] B. J. Pettis. On integration in vector spaces. Trans. Amer. Math. Soc. 44, 277-304 (1938).

[11] K. Pfaffelmoser. Global classical solutions of the Vlasov-Poisson system in three dimensions for general initial data. J. Differential Equations, 95 (1992), 281-303.

[12] G. Rein. Collisionless Kinetic Equations from Astrophysics - The Vlasov-Poisson System. Handbook of Differential Equations: Evolutionary Equations, Elsevier B.V., 3 (2007).

[13] J. Schaeffer. Global existence of smooth solutions to the Vlasov-Poisson system in three dimensions. Comm. Partial Differential Equations, 16 (1991), 1313-1335.

[14] E. Stein. Singular integrals and differentiability properties of functions. Princeton Univ. Press (1970).

[15] K. Yosida. Functional Analysis. Springer, 6 (1980). 



\section{Eidesstattliche Versicherung}

Hiermit versichere ich an Eides statt, dass ich die vorliegende Arbeit selbstständig verfasst und keine anderen als die von mir angegebenen Quellen und Hilfsmittel verwendet habe.

Weiterhin erkläre ich, dass ich die Hilfe von gewerblichen Promotionsberatern bzw. -vermittlern oder ähnlichen Dienstleistern weder bisher in Anspruch genommen habe, noch künftig in Anspruch nehmen werde.

Zusätzlich erkläre ich hiermit, dass ich keinerlei frühere Promotionsversuche unternommen habe. 
